\title{
Computational Investigation of Vortex Dynamics and Aerodynamic Performance in Flapping Propulsion
}

\begin{tabular}{c} 
A Dissertation \\
Presented to \\
the faculty of the School of Engineering and Applied Science \\
University of Virginia \\
\hline
\end{tabular}

In Partial Fulfillment of the

Requirements for the Degree

of

Doctor of Philosophy

By

Chengyu Li

May 2016 


\section{APPROVAL SHEET}

The dissertation is submitted in partial fulfillment of the

requirements for the degree of

Doctor of Philosophy

Chengyu Li, Author

This dissertation has been read and approved by the examining Committee:

Haibo Dong, Advisor

Eric Loth, Committee Chair

Christopher P. Goyne, Committee Member

Matthew A. Reidenbach, Committee Member

Baoxing $\mathrm{Xu}$, Committee Member

Accepted for the School of Engineering and Applied Science:

Craig H. Benson, Dean

School of Engineering and Applied Science

May 2016 


\begin{abstract}
Flapping motion is widely utilized in many biological propulsion systems, including insect/bird wings and fish pectoral fins. To survive through millions of years of evolution, these natural flyers/swimmers have developed superior and complex propulsive mechanisms to avoid predators and hunt for prey. However, achieving biological levels of aero/hydro-performance in bio-inspired robots design has proven elusive. This is due to our lack of understanding of the fundamental physics of deformable wings/fins and the technical difficulties in studying their complex locomotion.
\end{abstract}

The current dissertation focuses on two aspects of flapping wings. Firstly, we investigate the dominant flow control parameters that govern vortex development and aerodynamic performance using simplified canonical models. A Cartesian grid based immersed boundary incompressible Navier-Stokes solver is used to simulate the corresponding unsteady flows. The parametric study of 2-D flapping plates reveals that the rotational phase difference between the leading-edge and trailing-edge is the dominant parameter to achieve force enhancement. A moderate phase difference is able to feed extra circulation into the trailing-edge vortex which induces a stronger counterpart leading-edge vortex. As a result of local flow modulation, the vortex on the suction side is pulled down closer to the plate, which leads to the improvement of force production up to $26 \%$. Our 3-D flapping plates demonstrate that the phase difference between pitching and rolling motion is a critical parameter to achieve the optimal aerodynamic performance. This is because the phase difference directly alters the interaction between leadingedge vortices and trailing-edge vortices, and thus minimizes the wake deflection in the downstream direction. The simulation results show that an optimal phase difference can improve 
the cycle-averaged force and efficiency of up to $23 \%$ and $15 \%$, respectively. In addition, a unique vortex structure (named "double-C"-shaped vortex rings) produced by low-aspect-ratio flapping plates is first reported here. This vortex structure is found to be quite robust over a range of Strouhal numbers and Reynolds numbers.

Secondly, the wake topology and propulsive performance of real insect wings are examined via a combined experimental and computational approach. High-speed photogrammetry and accurate 3-D reconstruction are used to measure the deformable wing kinematics of freely flying dragonflies with precise detail. Then, flow simulations are conducted to evaluate the unsteady flow characteristics and the associated aerodynamic performance. The quantitative measurements of wing kinematics and surface deformation show that the phase difference between leading-edge and trailing-edge rotation observed in nature is in line with the optimal value we found in the aforementioned canonical model studies. Our flow simulations further reveal that the enhancement of aerodynamic functions can be achieved in two ways: 1) improving the power economy by preventing the tip vortex from bursting, and 2) improving the leading-edge vortex attachment by suppressing the generation of the secondary vortex. These findings have the potential to help us connect specific features in complex flapping locomotion with observed vortex dynamics and aerodynamic force production, so as to bring new insights into the design of high-efficient bio-inspired robotic systems. 


\section{Acknowledgements}

I would like to express my heartfelt gratitude and sincere thanks to my research advisor Dr. Haibo Dong, Associate Professor of Mechanical Engineering, Department of Mechanical and Aerospace Engineering at University of Virginia. Without his guidance, constant supervision, and valuable suggestions throughout the entire period of the work, it would have been impossible to complete the research work. I am thankful to my parents for all their support throughout my life. I am also greatly thankful to Dr. Geng Liu, Mr. Yan Ren, Dr. Samane Zeyghami, Dr. Zongxian Liang, Dr. Hui Wan, Mr. Ayodeji T. Bode-Oke, and all other current and previous Flow Simulation Research Group (FSRG) members for their assistance, generosity and advice throughout my Ph.D. study.

I am very grateful to the faculty and staff of the Department of Mechanical and Aerospace Engineering at University of Virginia for providing me with the opportunity to pursue higher studies. 


\section{Table of Contents}

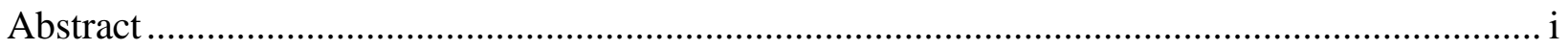

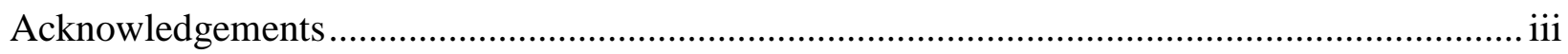

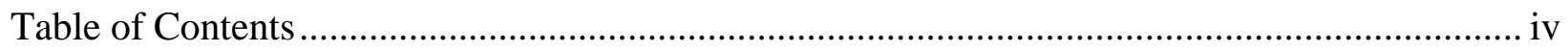

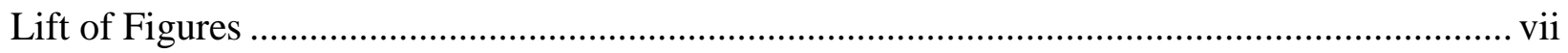

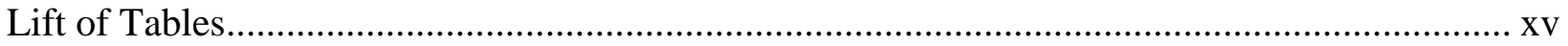

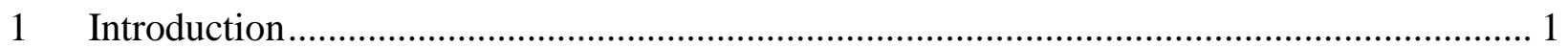

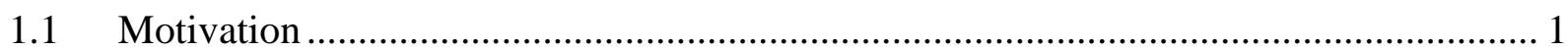

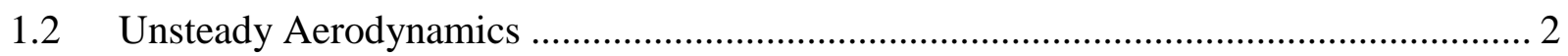

1.3 Vortical Structures of Oscillating Foils....................................................................... 3

1.4 Effect of Surface Morphing on Unsteady Aerodynamics ............................................... 7

1.5 Force Enhancement Mechanism in Tandem Flapping Wings ........................................ 9

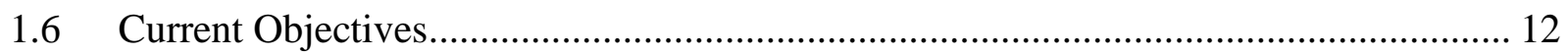

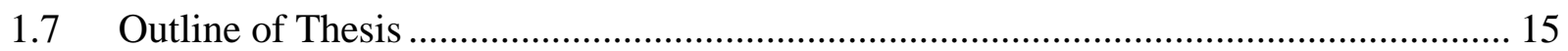

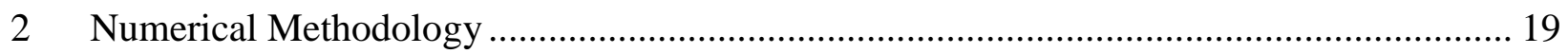

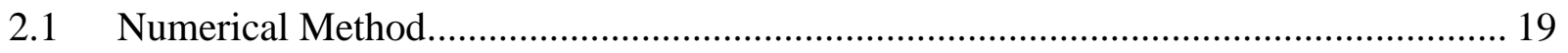

2.2 High-Speed Photogrammetry and Surface Reconstruction.......................................... 21

2.3 Proper Orthogonal Decomposition (POD) …………………................................. 24

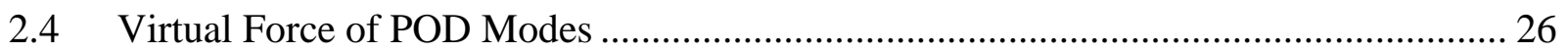

3 Effects of a Dynamic Trailing-Edge Flap in Flapping Motion............................................. 27

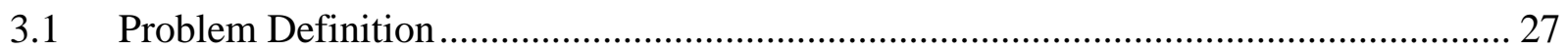

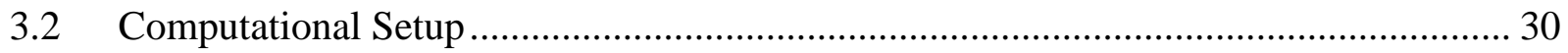

3.3 Aerodynamic Performance and Vortical Structure ………………............................... 33

3.3.1 Effect of TEF Deflection Phase Difference ........................................................... 35

3.3.2 Effect of TEF Deflection Amplitude …………….......................................... 43 
3.3.3 Effect of Hinge Location ............................................................................. 49

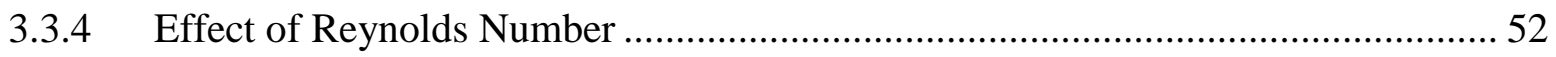

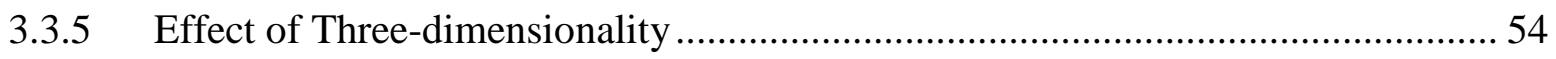

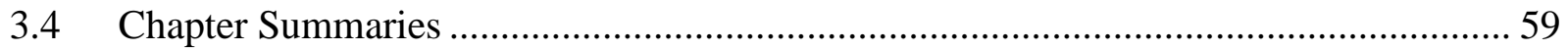

4 Wake Topology and Propulsive Performance of Bio-inspired Pitching-Rolling Plates ....... 60

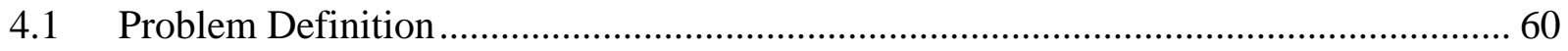

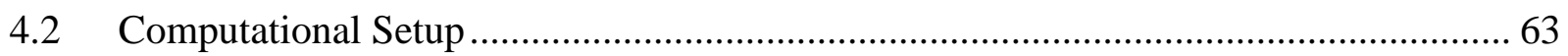

4.3 Wake Topology and Propulsive Performance ............................................................... 70

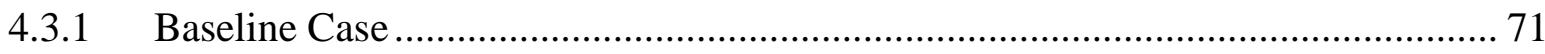

4.3.2 Effects of Strouhal Number ........................................................................... 79

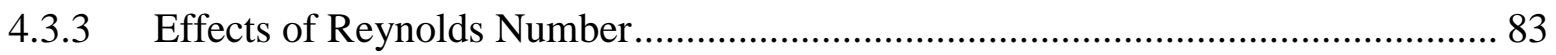

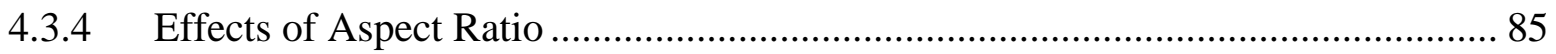

4.3.5 Effects of Phase Difference between Pitching and Rolling ................................... 88

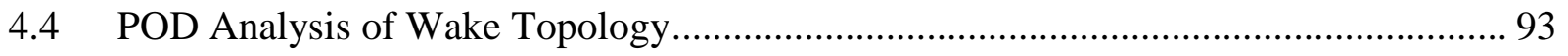

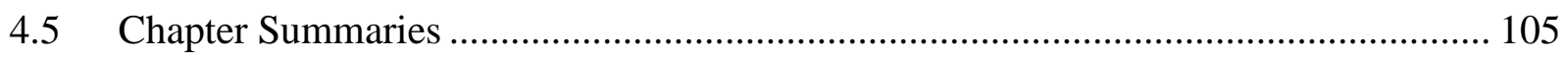

5 Aerodynamic Effects of Morphing Wings in Dragonfly Forward Flight ........................... 107

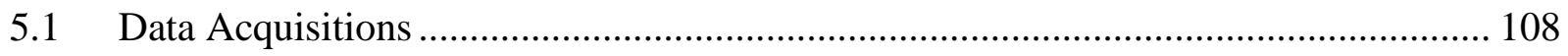

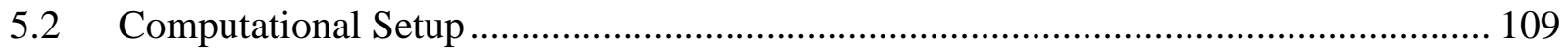

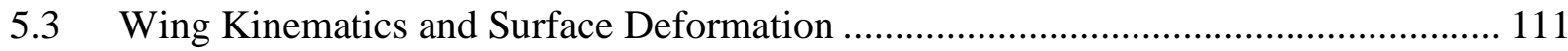

5.4 Aerodynamic Performance ................................................................................... 114

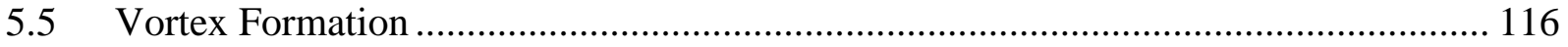

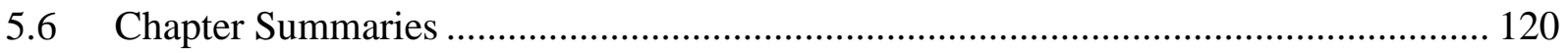

6 Aerodynamic Functions of Tandem Flapping Wings in Dragonfly Turning Maneuver .... 121

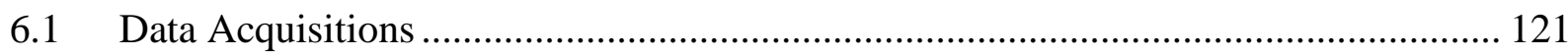

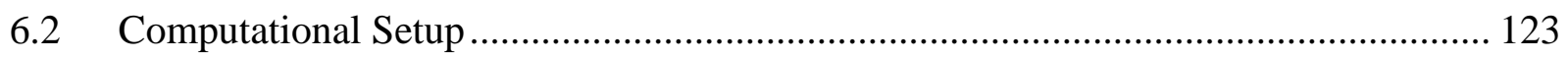




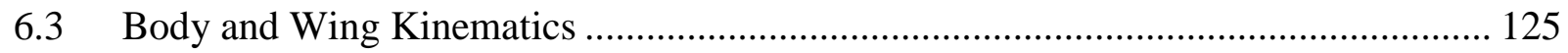

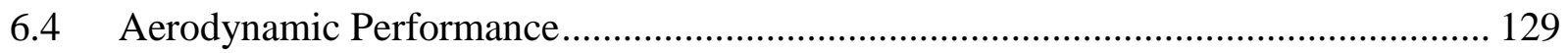

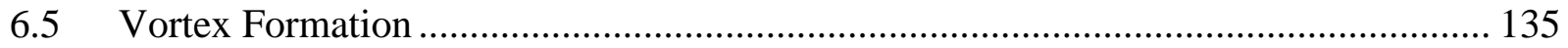

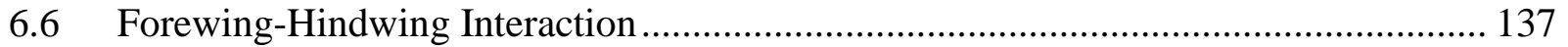

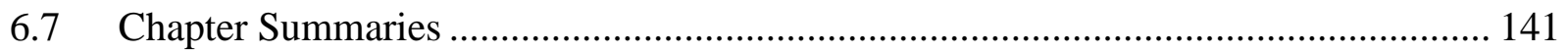

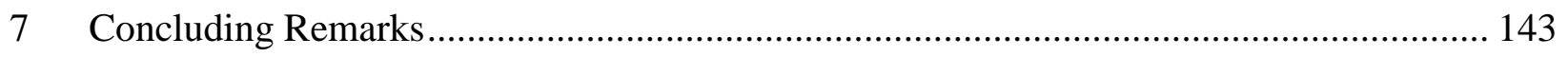

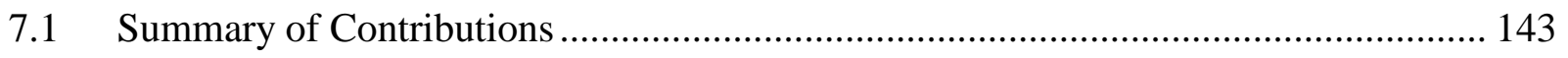

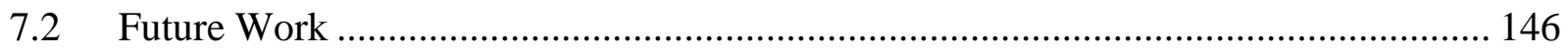

Appendix A: CFD Solver Validations …................................................................ 147

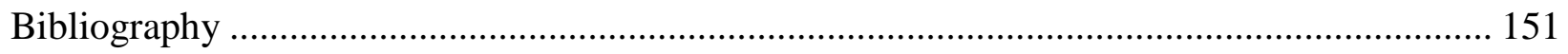




\section{Lift of Figures}

Figure 1-1. Maximum lift coefficient can be achieved by different type propulsive mechanisms

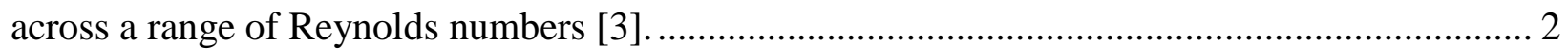
Figure 1-2. (a) Leading-edge vortex (LEV) on top of a fruit fly wing [5]. (b) Wake pattern during

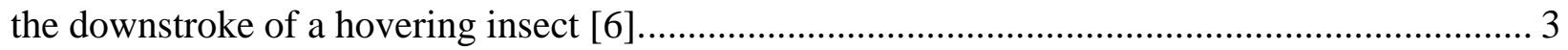
Figure 1-3. Wake transitions of flapping foils by increasing Strouhal number for $R e=255$ [16]. (a) von Kármán wake at $S t \approx 0.15$. (b) Inverse von Kármán wake at $S t \approx 0.25$. (c) Asymmetric

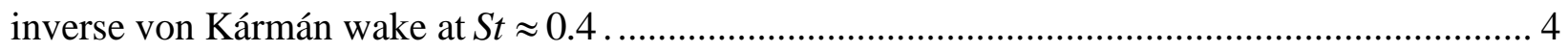
Figure 1-4. Vortical pattern in a rectangular foil with aspect ratio 3, oscillating at Strouhal number 0.18 (a) and 0.35 (b), respectively. The Reynolds number in both cases is about 200 [28].

Figure 2-1. Schematic of the ghost cell method for the immersed boundary treatment............. 21 Figure 2-2. (a) High-speed camera system set-up in the lab. (b) Conceptual model of the photogrammetry system used to capture insects in free flight based on Autodesk Maya........... 22 Figure 2-3. Initial configuration of a dragonfly template mesh. (a) Top-down image is showing the marker points on the wings. (b) Wing and body template models with the surface points corresponding to the top level of the subdivision hierarchy marked in red. 23

Figure 2-4. Reconstructed wings at a time step where a large amount of twist and camber is

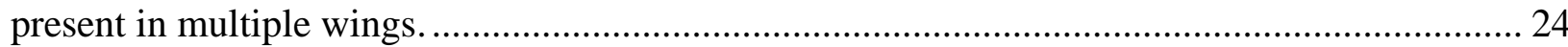

Figure 3-1. Schematics of a vein-stiffened wing and the idolized model for presenting the chordwise deflection $(\theta)$ around a possible pivot point. 27 Figure 3-2. (a) A schematic illustration of the specified kinematics parameters. The main plate and TEF are colored in black and red, respectively; (b) Definition of flap deflection amplitude ( $\beta_{T}$ ) and deflection phase difference $(\varphi)$; (c) Sample of positive camber configuration at $\beta_{T}=60^{\circ}, \varphi=60^{\circ}$; (d) Sample of negative camber configuration at $\beta_{T}=60^{\circ}, \varphi=-120^{\circ}$. 29

Figure 3-3: (a) Flapping plate configuration immersed in the 2-D non-uniform Cartesian grid. (b) Boundary conditions for the simulation. 31

Figure 3-4: Spatial and Temporal sensitivity analyses for a rigid flapping plate at $A_{0} / c=3.0$. Lift coefficient $(\mathrm{a}, \mathrm{c})$ and drag coefficient $(\mathrm{b}, \mathrm{d})$ time history for the $10^{\text {th }}$ flapping stroke. 32 
Figure 3-5. Time traces of lift (a), drag (b), and power (c) coefficients for fully rigid plate and plate with a TEF at $\beta_{T}=60^{\circ}, \varphi=60^{\circ}$ (positive camber) and $\beta_{T}=60^{\circ}, \varphi=-120^{\circ}$ (negative camber). The half-cycles representing down-stroke are shaded. 33 Figure 3-6. Time variation of relative force increments compared to the fully rigid plate. (a) $\beta_{T}=60^{\circ}, \varphi=60^{\circ}$. (b) $\beta_{T}=60^{\circ}, \varphi=-120^{\circ}$. 34

Figure 3-7. A series of instantaneous vortex field in a flapping cycle for a fully rigid plate (first and fourth columns), TEF deflection at $\beta_{T}=60^{\circ}, \varphi=60^{\circ}$ (second and fifth columns) and TEF deflection at $\beta_{T}=60^{\circ}, \varphi=60^{\circ}$ (third and sixth columns). The blue and red colors indicate clockwise and counterclockwise vortices, respectively. 36 Figure 3-8. Comparison of LEV (a) and TEV (b) circulation. The vortex circulation is nondimensionalized by $U_{\max } c$. The positive and negative values correspond to the strength of the vortex during down-stroke and up-stroke, respectively. (c-d) Vortex contour at an instant labeled by the dashed-dot line in (a) for the fully rigid plate (black color), the TEF deflection at $\beta_{T}=60^{\circ}, \varphi=60^{\circ}$ (red color), and the TEF deflection at $\beta_{T}=60^{\circ}, \varphi=-120^{\circ}$ (blue color). The fully rigid and deformed plates are shown as gray and green color, respectively. The solid and dashed line indicates the LEV and TEV, respectively. 38 Figure 3-9. Comparison of pressure distribution and velocity vector at $t / T=19.75$. (a) Fully rigid plate, (b) Positive cambered plate at $\beta_{T}=60^{\circ}, \varphi=60^{\circ}$, and (c) Negative cambered plate at $\beta_{T}=60^{\circ}, \varphi=-120^{\circ}$. The pressure is normalized by $\rho U_{\max }^{2}$

Figure 3-10. Far wake vortical structure comparison of fully rigid plate (a), TEF deflection at $\beta_{T}=60^{\circ}, \varphi=60^{\circ}$ (b) and TEF deflection at $\beta_{T}=60^{\circ}, \varphi=-120^{\circ}$ (c) at $\mathrm{t} / \mathrm{T}=20.0$. The dashed square corresponds to the region shown in Figure 3-7. 42 Figure 3-11. Time traces of lift (a-b), drag (c-d), and power (e-f) coefficients for positive and negative camber formations at different deflection amplitudes. 44 Figure 3-12. Far wake vortical structure comparison of TEF deflection at $\beta_{T}=30^{\circ}, \varphi=60^{\circ}$ (a), $\beta_{T}=30^{\circ}, \varphi=-120^{\circ}(\mathrm{b}), \beta_{T}=90^{\circ}, \varphi=60^{\circ}(\mathrm{c})$, and $\beta_{T}=90^{\circ}, \varphi=-120^{\circ}(\mathrm{d})$ 45

Figure 3-13. Aerodynamic performance of plate at $H_{L}=0.75 c$. Cycle-averaged lift (a), drag (b), and power (c) coefficients, and lift-to-power ratio (d). 46 
Figure 3-14. Definition of effective pitching angle $\left(\alpha_{\text {eff }}\right)$....

Figure 3-15. Aerodynamic performance of plate has different hinge locations. Cycle-averaged lift (a), drag (b), and power (c) coefficients, and lift-to-power ratio (d). 51 Figure 3-16. Aerodynamic performance of plates with various deflection phase difference at $\beta_{T}=60^{\circ}$ for different Reynolds numbers. Cycle-averaged lift (a), drag (b), and power (c)

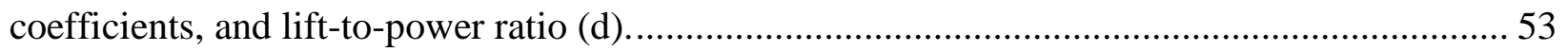
Figure 3-17. Comparison of aerodynamic performance between 2-D plate and 3-D plate $(A R=2)$ at $\beta_{T}=60^{\circ}$ for different TEF deflection phase difference. Cycle-averaged lift (a), drag (b), and

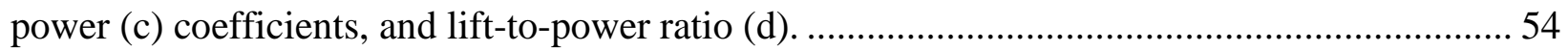
Figure 3-18. Comparison of instantaneous lift (a-b) and drag (c-d) coefficients between the 2-D and 3-D cases for positive and negative camber formations. 55

Figure 3-19. Comparison of vortical structures between positive $\left(\beta_{T}=60^{\circ}, \varphi=60^{\circ}\right)$ and negative $\left(\beta_{T}=60^{\circ}, \varphi=-120^{\circ}\right)$ camber formations at selected instants during the down-stroke of the $4^{\text {th }}$

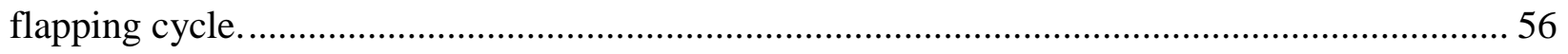
Figure 3-20. Comparison of Z-vorticity contours between positive $\left(\beta_{T}=60^{\circ}, \varphi=60^{\circ}\right)$ and negative $\left(\beta_{T}=60^{\circ}, \varphi=-120^{\circ}\right)$ camber formation at selected time instants during the downstroke of the $4^{\text {th }}$ flapping cycle. The slices cuts are taken at plate tip, at a distance of $0.5 c$ from the tip, and at the mid-span. 58 Figure 4-1. Schematic of the plate kinematics during the upstroke (a) and the downstroke (b), respectively. 61

Figure 4-2. The time histories of plate rotation during an entire cycle. The black dash-dot line denotes six moments at $\mathrm{t} / \mathrm{T}=3.875,3.9375,4.0,4.125,4.1875,4.25,4.3125,4.375$, and 4.4375, respectively, for the illustration of the "double C" loop formation during a half cycle in Figure 4-6.

Figure 4-3. Typical grid employed in the current simulations for the circular plate with $A R=1.27$ $(S / c=1.0)$. The grid is $235 \times 233 \times 113$, and the domain size is $30 c \times 25 c \times 25 c$

Figure 4-4. Comparison of computed profiles in the wake demonstrating the grid independence of the computed results for the $A R=1.27, S t=0.6$ and $R e=200$ case. Five profiles at $\mathrm{x} / \mathrm{c}=1,2,3,4$ and 5 are shown. (a) Streamwise $\left(\bar{u}_{1}-U_{\infty}\right)$ velocity profiles. (b) Transverse $\left(\bar{u}_{2}\right)$ velocity 
profiles. (c) Spanwise $\left(\bar{u}_{3}\right)$ velocity profiles. (d) Fluctuation kinetic energy $\left(\overline{u_{1}^{\prime 2}}+\overline{u_{2}^{\prime 2}}+\overline{u_{3}^{\prime 2}}\right) / 2$ at the center of the XY-plane....

Figure 4-5. Slides of 2-D contours of streamwise vorticity at $t / T=4.25$ in the near wake (a) and far wake (b), respectively, for measuring the relative changes of vortical structure at different spatial and temporal grid. The corresponding section-cutting locations can be found in Figure 4-8

(c) and (f).

Figure 4-6. The wake topology of a flapping circular plate $(A R=1.27)$ with $S t=0.6$ and $R e=200$ at $\mathrm{t} / \mathrm{T}=3.875,3.9375,4.0,4.125,4.1875,4.25,4.3125,4.375$, and 4.4375 , respectively. In (a-c), the plate is rolling downward and reaches to the lowest point at $\mathrm{t} / \mathrm{T}=4.0$; in $(\mathrm{d}-\mathrm{f})$, the plate is rolling upward and reaches to the middle point at $\mathrm{t} / \mathrm{T}=4.25$; in $(\mathrm{g}-\mathrm{i})$, as the plate continuous rolling upward, the newly formed double-C shaped ring gradually shed our from the plate. The time history of the corresponding rolling and pitching angles are shown in Figure 4-2. The iso-surface

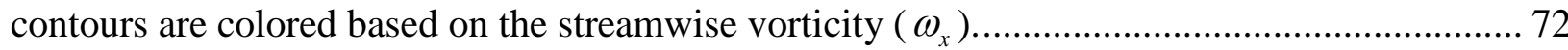
Figure 4-7. Wake topology of a pitching-rolling circular plate $(A R=1.27)$ after four flapping cycles. The plate is at the phase where it is in the center position of its rolling motion and about to move upward (t/T=4.25). (a) Perspective view, (b) side view, and (c) top view. The vortex loops are labeled based on their shedding sequence, and the propagation directions of the rings are indicated by red arrows in (b).... 75

Figure 4-8. Slides of the contours of streamwise vorticity $\left(\omega_{x}\right)$ at $\mathrm{t} / \mathrm{T}=4.25$. (a-d) the vortical structure of $R_{8}$, which consists of vortex $V_{1} \sim V_{4}$. (e-f) the vortical structure of $R_{7}$, which consists of vortex $V_{5} \sim V_{8}$. The vortex interactions occurring between $R_{7}$ and $R_{6}$ are shown in (g-h), and the interactions between $R_{5}$ and $R_{6}$ are presented in (i). In the far wake, the double-Cshaped ring gradually evolves into a single-loop vortex $(\mathrm{j}-1)$. 77 Figure 4-9. Computed time-varying force coefficients for the circular plate $(A R=1.27)$ at $R e=200$ and St=0.6. (a) Thrust coefficient, (b) lift coefficient, and (c) spanwise force coefficient. The black dash-dotted lines indicate the corresponding cycle-averaged value. The pitching and rolling motion curves of the plate are also superimposed with the force history. Figure 4-10. Wake topology for the $A R=1.27$ circular plate during the phase where the plate is at the middle point in its up stroke. The left and right columns show the side view and top view, 
respectively. (a-b) The side view and top view of the case of $S t=0.4$. (c-d) The side view and top view of the case for $S t=0.8$. The Reynolds number is 200 for all these cases. 80 Figure 4-11. Variation in cycle-averaged hydrodynamic performance for the baseline case with changing Strouhal number. The red and blue curves indicate the mean thrust coefficient and propulsive efficiency, respectively. 82 Figure 4-12. Vortex topology of a circular plate $(A R=1.27)$ for different Reynolds numbers. (a) $R e=100$. (b) $R e=400$. The Strouhal number is 0.6 in both cases. 84 Figure 4-13. Computed time-varying force coefficients for the circular plate $(A R=1.27)$ at various Reynolds numbers. (a) Thrust coefficient, (b) lift coefficient, and (c) spanwise force coefficient. The Strouhal number is 0.6 for all of these cases. 85 Figure 4-14. Vortex topology for an elliptical plate with $A R=1.91$ (a) and $A R=2.55$ (b). For each case, the Strouhal number and Reynolds number are 0.6 and 200, respectively. 86 Figure 4-15. Comparison of 2-D vorticity contours for $A R=1.27$ (a, d), $A R=1.91$ (b, e) and $A R=2.55$ (c, f). Figures (a-c) are the streamwise vorticity $\left(\omega_{x}\right)$ contours at the location near the plate trailing edge and (d-f) are the spanwise vorticity $\left(\omega_{z}\right)$ contours at the mid-span of the plates. The spatial locations of the slice cuts are shown as red planes in (a) and (d), respectively. 87 Figure 4-16. Computed time-varying force coefficients for the plate with various aspect ratios $(A R)$. (a) Thrust coefficient, (b) lift coefficient, and (c) spanwise force coefficient. The Strouhal number and Reynolds number are fixed at 0.6 and 200, respectively, for all these cases. 88 Figure 4-17. Effects of phase difference between pitching and rolling motions on the wake structure for the $A R=1.27$ circular plate. (a) $\psi=100^{\circ}$. (b) $\psi=110^{\circ}$. (c) $\psi=120^{\circ}$. The left and right columns show the side view and top view, respectively. The Strouhal number, Reynolds number and aspect-ratio are fixed at 0.6, 200 and 1.27, respectively, for all these cases. .......... 91 Figure 4-18. Computed time-varying force coefficients for the plate with various phase difference between pitching and rolling motion $(\psi)$. (a) Thrust coefficient, (b) lift coefficient, and (c) spanwise force coefficient. The Strouhal number and Reynolds number are fixed at 0.6 and 200, respectively, for all these cases.

Figure 4-19. Variation in the cycle-averaged hydrodynamic performance for the baseline case $\left(\psi=90^{\circ}\right)$ as a function of phase difference between pitching and rolling motion. The red and 
blue curves indicate the mean thrust coefficient and propulsive efficiency, respectively. The Reynolds number and Strouhal number for all these cases are set to 200 and 0.6, respectively.. 92 Figure 4-20. Wake topology of circular plate ( $\mathrm{AR}=1.27)$ with $\mathrm{St}=0.6$ and $\mathrm{Re}=200$ at two different phase. (a) The plate is at the lowest point in its rolling motion and starting to move upward $(\mathrm{t} / \mathrm{T}=4.0)$. (b) The plate is at the highest point in its rolling motion and starting to move downward $(\mathrm{t} / \mathrm{T}=4.5)$. To easily identify the vorticity transpose direction along $\mathrm{x}$-axis, the vortex ring $(\mathrm{Q}=0.2)$ is colored by the streamwise vorticity $\left(\omega_{x}\right)$. 94 Figure 4-21. Iso-surfaces of Q-criterion of mean vorticity colored by the X-direction vorticity ( $\left.\bar{\omega}_{x}\right)$ 95

Figure 4-22. Normalized eigenvalues (red circle) and captured energy by the first $i$ modes (blue square) versus mode number $i=1,2, \ldots, 24$. 96 Figure 4-23. Iso-surface of Q-criterion of POD modes from 1 to 8. The iso-surface of the modes are colored by the $\mathrm{X}$-direction vorticity. 100 Figure 4-24. The history of the temporal coefficient and phase portraits of the coefficients respect to $\alpha_{1}$ 102

Figure 4-25. Thrust, lift, and spanwise force coefficients (a-c) from simulation results and its corresponding power spectrum (d-f). 103

Figure 4-26. Thrust, lift, and spanwise force coefficients of $G_{1}(\mathrm{a}-\mathrm{c})$ and $G_{-1}(\mathrm{~d}-\mathrm{f})$ comparing with the DNS results (black line). 104

Figure 4-27. Reconstructed flow fields of $G_{1}(\mathrm{a})$ and $G_{-1}$ (b) at $\mathrm{t} / \mathrm{T}=7.0, \mathrm{Q}=0.2$ 105 Figure 5-1. Flexible wing kinematics and computational setup. (a) Chord-lines of dragonfly forewing (dashed line) and hindwing (solid line) at a few instances. (b) Grid and domain employed for the cruising dragonfly simulations.

Figure 5-2. Definition of wing orientation angles, wing stroke angle $(\psi)$, wing deviation angle $(\phi)$, wing pitch angle $(\theta)$, and camber deformation $(\mathrm{h} / \mathrm{c}) . \mathrm{r}$ is the distance from wing root to reference point, meanwhile $\mathrm{R}$ is the length from wing root to wing tip. 113 Figure 5-3. Time course of wing kinematics of dragonfly hindwing. (a) Wing stroke angle $(\psi)$ and wing deviation angle $(\phi)$. (b) and (c) are the wing pitch angles $(\theta)$ and camber deromation 
(h/c) of different sections along the wing span, respectively. The downstroke period is shaded as a gray color.

Figure 5-4. Time course of lift (a), thrust (b) and power (c) of the hindwing.

Figure 5-5. Comparison of vortex formation along the wing span at the mid-downstroke (t/T=0.31). (a) Original wing. (b) Twist-only wing (c) Rigid wing. The vortical structure including leading-edge vortex (LEV), trailing-edge vortex (TEV), and secondary vortex (SV) are labeled. 116

Figure 5-6. Comparison of LEV circulation along the wing span at the mid-downstroke $(\mathrm{t} / \mathrm{T}=0.31)$. The vortex circulation is nondimensionalized by $U_{\infty} \bar{c}$. 117

Figure 5-7. Comparison of 3-D vortical structure and wing surface aerodynamic power consumption contour of the hindwing at the mid-down stroke $(\mathrm{t} / \mathrm{T}=0.31)$. (a) Original wing model. (b) Twist-only wing model. (c) Rigid wing model. 118

Figure 5-8. Comparison of top surface pressure contours (top row) and the corresponding 2-D flow fields at the $70 \%$ wing span (bottom row) when the hindwing experienced the maximum camber formation ( $\mathrm{t} / \mathrm{T}=0.42$ ). (a) Original wing model. (b) Twist-only wing model. (c) Rigid wing model.

Figure 6-1. Motion reconstruction of dragonfly taking-off maneuver. The side panels show 4 of 110 frames recorded by high-speed videography.

Figure 6-2. (a) Grid and domain employed for the dragonfly maneuver simulations. (b) Highdensity surface mesh with about 3000 triangular elements used to define each wing.

Figure 6-3. Dragonfly body and wing coordinate systems. (a) The body frame $\left(x_{b}, y_{b}, z_{b}\right)$ has its origin at the center of mass. (b) Defination of wing kinematics parametters. 126 Figure 6-4. Time course of body and wing kinematics of a maneuvering dragonfly. (a) Dragonfly body orientation and path velocity. (b) Wing stroke, deviation, and pitch angles. 128 Figure 6-5. Time course of the absolute value of wing tip velocity for forewings (a) and hindwings (b). The downstroke of each cycle is shaded. Figure 6-6. Time course of aerodynamic forces of forewings (a, c) and hindwings (b, d), respectively. The downstroke of each cycle is shaded. 130 Figure 6-7. Time course of aerodynamic torques of forewings (a, c, e) and hindwings (b, d, f), respectively. 132 
Figure 6-8. Mean values of torques varying with each half cycle of forewings (a) and hindwings (b), respectively

Figure 6-9. Time course of specific aerodynamic power consumption of forewings (a) and hindwings (b), respectively. 134

Figure 6-10. Time-averaged surface pressure distribution projected on the vertical direction (a) and horizontal direction (b). 135

Figure 6-11. 3-D vortex structures in the flow during the early turning motion, where the time stamp from (a) to (d) is 23, 29, 35 and $40 \mathrm{~ms}$. The vortex loop from the downstroke is marked by dashed lines. 136

Figure 6-12. Comparison of instantaneous vertical force between the two-pair wings simulation and single-pair wings simulation. (a) left-forewing. (b) left-hindwing. (c) right-forewing. (d) right-hindwing. 138 Figure 6-13. Comparison of instantaneous horizontal force between the two-pair wings simulation and single-pair wings simulation. (a) left-forewing. (b) left-hindwing. (c) rightforewing. (d) right-hindwing. 139 Figure 6-14. Comparison of instantaneous specific power between the two-pair wings simulation and single-pair wings simulation. (a) left-forewing. (b) left-hindwing. (c) right-forewing. (d) right-hindwing. 140 Figure A-1. Lift and drag coefficients from the current simulation, from experimental (EXP) measurement [76] and from a viscous vortex particle method (VVPM) simulation [77]. 148 Figure A-2. Vorticity field at the labeled instants with 40 contour levels distributed uniformly between -20 and 20. The top row is from Ref. [77], and the bottom row is from the current simulation. 149 Figure A-3. Benchmarking of three-dimensional flapping simulation. The experimental and numerical data are reproduced from fig.3 C, D of Sane and Dickinson [126] and fig.4 C, D of Sun and Lan [58], respectively. 150 


\section{Lift of Tables}

Table 3-1: The maximum camber-to-chord ratio $\left(h_{\max } / c\right)$ and the timing of maximum camber $\left(\tau_{h \max }\right)$ during downstroke for real insects along with the optimal lift case from the current study.

Table 4-1. Parameters involved in the current study and their ranges.

Table 4-2. Results of spatial and temporal grid independence study for $A R=1.27, S t=0.6$ and $R e=200$ case. 66

Table 4-3. Comparison of computed distances of vortex paired in the near wake and far wake between different grid resolutions. 70

Table 4-4. Comparison of the wake oblique angle $(\alpha)$ and vortex ring orientation angle $(\beta) .82$ Table 4-5. Effects of phase difference on the wake oblique angle $(\alpha)$ and wake deflection angle $(\gamma)$ at $S t=0.6$ and $R e=200$. 89

Table 4-6. Contribution of the first eight eigenvalues to the total fluctuating kinetic energy...... 97

Table 4-7. Vorticity symmetry of the mean flow and POD modes. ........................................ 98

Table 5-1. Morphological data for the dragonfly in the current study................................... 109

Table 5-2. Comparison of mean lift $(\mathrm{L})$, thrust $(\mathrm{T})$, total force $(\mathrm{F})$, power $(\mathrm{P}), \mathrm{L} / \mathrm{P}$ and F/P of the

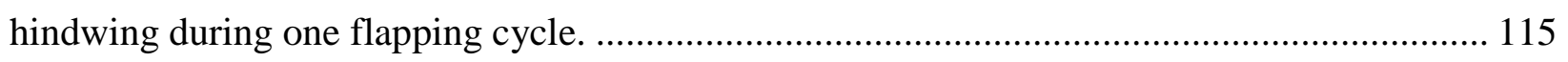

Table 6-1. Morphological data for the dragonfly in the current study................................. 122 Table 6-2. The effects of forewing-hindwing interaction on the mean aerodynamic forces and power. 141 


\section{Introduction}

\subsection{Motivation}

Observing insect and bird flight inspired the idea of designing man-made flying vehicles using the flapping-wing mechanism. Millions of years of natural selection have equipped these flyers with superior and complex propulsive mechanisms optimized for avoiding predators and capturing prey. It has been a long-standing effort to transform the flight strategies applied by natural flyers into our engineering applications for designing the next generation of micro air vehicles (MAVs). Compared to the conventional fixed- and rotary-wing configurations, the flapping-wing mechanism can offer more aerodynamic benefits, especially when flying in a relatively low Reynolds number regime $\left(10^{2}<\operatorname{Re}<10^{4}\right)$.

To illustrate flapping-wing mechanism advantages, Figure 1-1 provides an overview of the maximum lift that can be achieved by different flight methods across a range of Reynolds numbers. The grey-shaded area represents data reported in the steady flow. The remaining area represents unsteady experimental measurements. At high Reynolds numbers $\left(\operatorname{Re}>10^{5}\right)$, separated flow quickly becomes turbulent and generates sufficient lift in the steady flow condition. This flow feature allows fixed- and rotary-wing mechanisms to produce a high level of force at high Reynolds numbers. However, as the Reynolds number gradually decrease below $10^{4}$, the maximum lift produced in the steady flow becomes limited. Performance deteriorates chiefly because at low Reynolds numbers, the boundary layer remains laminar on the airfoil, making it susceptible to flow separation as the angle of attack increase [1]. In addition, due to the low aspect ratio wings, the tip vortex covers a major part of the wing, and the tip vortices shed affecting the aerodynamic performance [2]. Therefore, understanding the unsteady aerodynamics 
of flapping wings is critical to overcoming steady flow limitations and improving future MAVs designs.

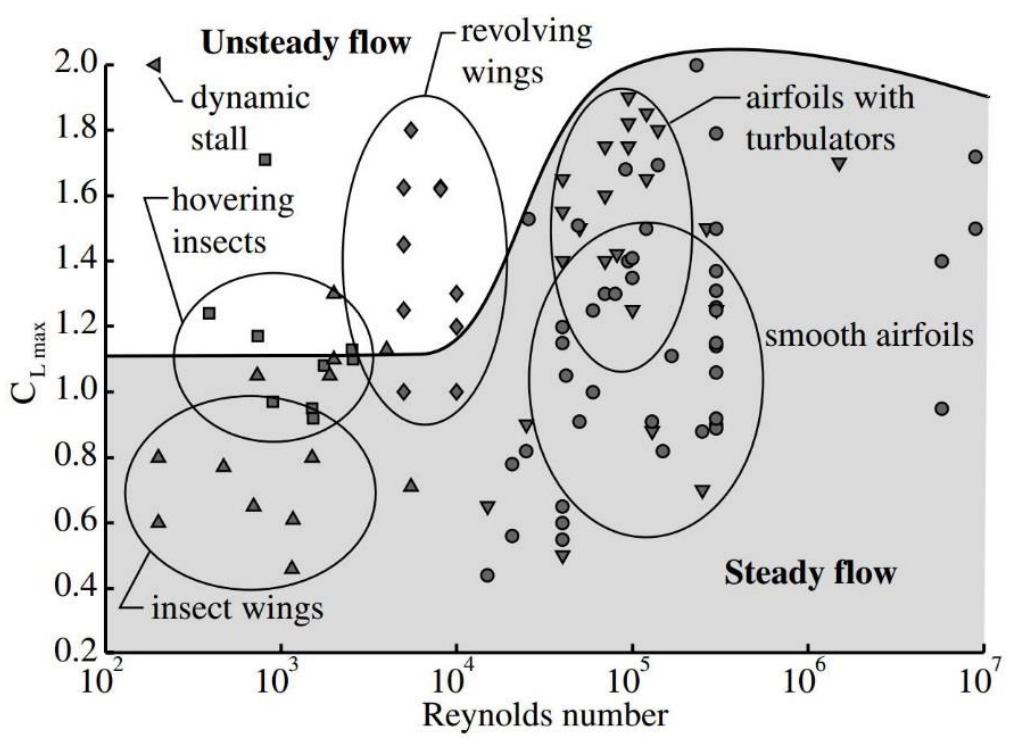

Figure 1-1. Maximum lift coefficient can be achieved by different type propulsive mechanisms across a range of Reynolds numbers [3].

\subsection{Unsteady Aerodynamics}

To achieve highly efficient flight in a low Reynolds number regime, insects and birds operate their wings with a combination of translation and rotation in the stroke plane. The dominant unsteady flow feature assumed to be responsible for aerodynamic lift is a leading edge vortex (LEV) produced by a laminar flow separation near the leading edge of the wing (Figure 1-2 (a)). This vortical structure produces a region of low pressure near the wing surface and influences the strength of the bound circulation about the wing. Ellington et al. [4] first illustrated the direct evidence of the existence of LEV by visualizing the flow around a threedimensional (3-D) robotic wing at Reynolds number around $10^{3}$. This LEV was observed similar to the vortical structure produced during dynamic stall observed for conventional configurations 
undergoing rapid pitch or plunge maneuvers. However, unlike the vortex produced during a dynamic stall, the leading edge vortex was not shed even after traveling many chord length of distance. As the flapping wing translate in its stroke plane, a spanwise velocity gradient interacted with the leading-edge vortex. This caused the axial flow to spiral toward the wing tip direction. The axial flow transported momentum out of the vortex, keeping the leading-edge vortex attached and stable. The LEV began to detach at the section close to the wing tip and shed into the wake. The vortex system generated by the flapping wing in its surrounding fluid induces downwash, forming a coherent momentum jet to maintain sustained flight (Figure 1-2 (b)).

(a)

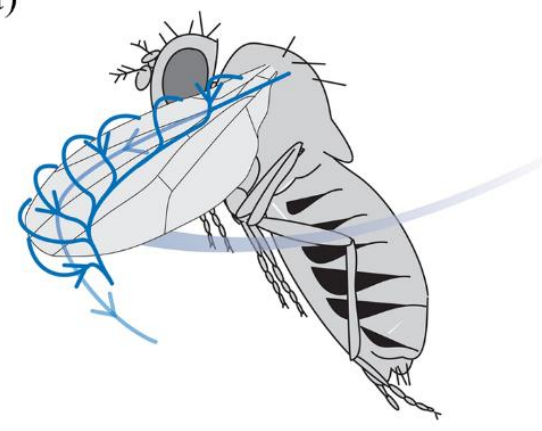

(b)

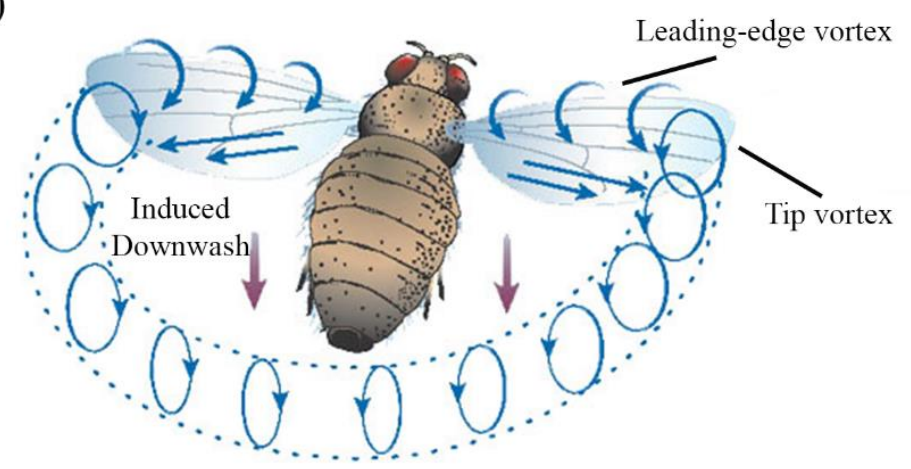

Figure 1-2. (a) Leading-edge vortex (LEV) on top of a fruit fly wing [5]. (b) Wake pattern during the downstroke of a hovering insect [6].

\subsection{Vortical Structures of Oscillating Foils}

According to fluid dynamics, studying vortex structures generated by flapping wings can help us reveal the flow physics behind their complex flapping kinematics. It is because that the flapping flight usually operates in a low Reynolds number regime, where vortices are dominant flow features [7]. In addition, the unsteady force generation can be related to the vortex formation directly or indirectly. 
For these reasons, the associated wake structures of simplified flapping kinematics have been studied experimentally $[8,9,10]$ and computationally $[11,12,13,14,15]$. In previous studies, this flapping motion was simplified as a two-dimensional (2-D) pitching and/or heaving problem by assuming that the wing's aspect ratio was sufficiently large. An increase in Strouhal numbers $(S t)$ has been found to induce a transition of the wake pattern from a von Kármán vortex street (Figure 1-3 (a)) to an inverse von Kármán vortex street that characterizes propulsive wakes (Figure 1-3 (b)). At higher Strouhal numbers, the shed vortex pairs propagate at an angle to the streamwise axis, and the inverse von Kármán vortex pattern gives rise to an asymmetric wake $[16,17,18,19,20]$, as shown in Figure 1-3 (c). However, for finite-aspect-ratio plates undergoing identical motions, the asymmetric wakes are absent due to the suppression of vortex coupling by the three dimensionality and the symmetric circulation distribution along the interconnected vortex loops [21].
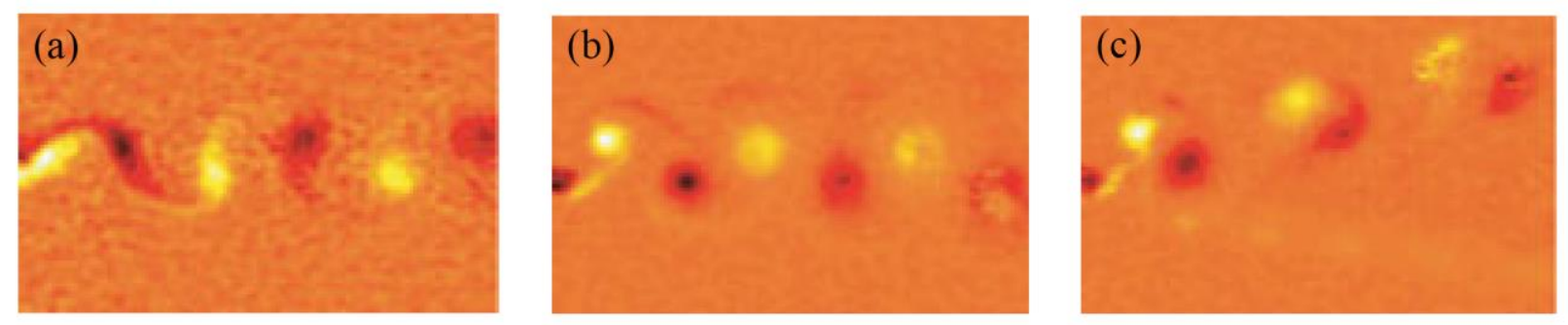

Figure 1-3. Wake transitions of flapping foils by increasing Strouhal number for $R e=255$ [16]. (a) von Kármán wake at $S t \approx 0.15$. (b) Inverse von Kármán wake at $S t \approx 0.25$. (c) Asymmetric inverse von Kármán wake at $S t \approx 0.4$.

Numerous studies have been conducted to understand the wake structures and the propulsive performance of finite-aspect-ratio plates undergoing pitching and/or heaving motions. In particular, flow visualization was used by von Ellenrieder et al. [22] to investigate the wake 
structure produced by low-aspect-ratio plates $(A R=3)$ under pitching-heaving motion at $R e=164$ and Strouhal numbers from 0.2 to 0.4 . The wake model in their work indicated that a pair of merged vortex rings shed each half cycle, forming a zig-zag chain (Figure 1-4 (a)). This observation was then numerically verified by Blondeaux et al. [23] using a low Strouhal number $(S t=0.175)$ flapping plate. However, at a higher Strouhal number $(S t=0.35)$, they found that the flow wakes consisted of two sets of vortical structures (Figure 1-4 (b)). A similar wake pattern transition was reported by Buchholz and Smits [24] in a study on low-aspect-ratio pitching panels $(A R=0.54)$. Using dye visualization and digital particle image velocimetry (DPIV), a chain of vortical structures was observed at $S t=0.23$ and $R e=640$. As the Strouhal number increased, the wake began to bifurcate and form two branches of horseshoe-like vortices. Further study [25] revealed that although distinct wake structures were observed at varying Strouhal numbers, the key features of the wake pattern are highly robust, even for Reynolds numbers on the order of $10^{4}$. In the meantime, Dong and his colleagues $[26,27]$ numerically studied the wake topologies of pitching-heaving ellipsoidal foils using a broader range of parameters including aspect ratios between 0.64 and 5.09, Strouhal numbers between 0.4 and 1.2, and Reynolds numbers in the range of $100 \leq R e \leq 400$. Similar bifurcated wake structures have been observed and found to be robust for all low aspect ratios.

The aforementioned 2-D and 3-D pitching-heaving studies have provided insights into the unsteady aero- and hydro-dynamic mechanisms applied in insect flight and fish swimming. When a rigid foil undergoes a pitching-heaving motion, it creates a symmetric leading-edge vortex due to the unchanged instantaneous effective angles of attack along the foil span. However, when a foil performs a combined pitching and rolling motion about a fixed hinge, the effective angle of attack with respect to the incoming flow varies along the foil span. As a result, 
an asymmetric leading-edge vortex with an increase in strength from the foil root to its tip forms and sheds during the pitching-rolling motion. However, the fundamental flow physics and wake structures of low-aspect-ratio pitching-rolling foils have not been fully investigated. Only a few studies can be found on bio-inspired pitching-rolling propulsors, and most of them have focused on the propulsive performance.

(a)

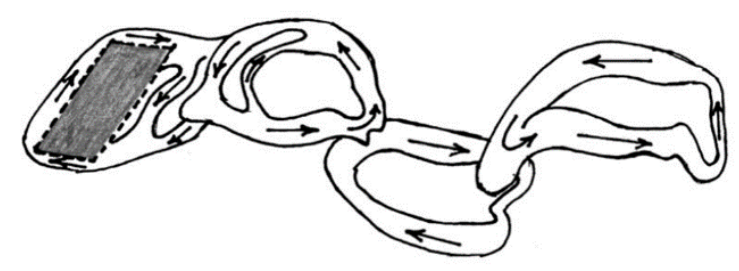

(b)

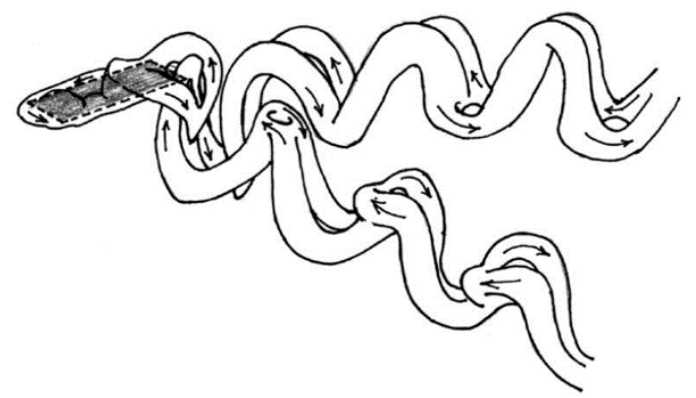

Figure 1-4. Vortical pattern in a rectangular foil with aspect ratio 3, oscillating at Strouhal number 0.18 (a) and 0.35 (b), respectively. The Reynolds number in both cases is about 200 [28].

For instance, Techet [29] experimentally studied a robotic pitching-rolling foil with an NACA 0012 cross section and found that the high thrust and high propulsive efficiency of the foil could only be achieved when the foil flapped with a smaller maximum angle of attack $\left(\alpha_{\max }=30^{\circ}\right)$ and high Strouhal number $(S t=0.6)$. The high rolling amplitude did not appear to improve the thrust but could reduce the power losses. In another experimental study, Bandyopadhyay et al.[30] explored low-aspect-ratio flapping fins $(A R=3)$ at chord Reynolds numbers between $3.6 \times 10^{3}$ and $1.5 \times 10^{5}$ in a tow tank. The authors demonstrated that lowaspect-ratio pitching-rolling fins can only produce thrust within a bounded Strouhal number 
$(0.2<S t<1.5)$ and that optimal efficiency can be achieved by tailoring the Strouhal number and pitch amplitude.

In addition to these experimental studies, the wake structures of bio-inspired propulsion have been discussed only in relation to a flow simulation of fish pectoral fins (Dong et al.[31] and Liu et al.[32]) and rigid foil with an aspect ratio of 2.55 (Bozkuttas[33]). These works indicated the existence of distinct flow features differing from those of the aforementioned pitching and/or heaving plates. In particular, the strong spanwise flow caused by the rolling motion resulted in the formation of stronger leading-edge and tip vortices, and the interactions among those vortices formed substantially more complicated vortex structures that convected into the wake.

\subsection{Effect of Surface Morphing on Unsteady Aerodynamics}

Along with the aforementioned unsteady aerodynamic phenomenon, surface morphing is another key feature of natural flapping wings. Natural flyers typically have flexible wings to adapt to the flow environment. Birds have different layers of feathers that are often connected to each other. Hence, they can adjust wing plane-form to fit specific flight modes. Bats have more than two dozen independently controlled joints in their wings and deformable bones that enable them to fly at positive and negative angle of attack to dynamically change wing camber. Insect wings differ from those of vertebrates in that they lack internal musculature extending into the aerodynamic surface of the wing. Thus, insects have little active control over wing properties,

and most deformations are a product of the passive mechanical properties of the wing interacting with the inertial and aerodynamic forces it generates while flapping. Both active and passive 
deformable wings are believed to have the potential to increase aerodynamic performance comparing with completely rigid wings in flying.

Recently, increasing attention has been paid to the aerodynamics of deformable wings. Several studies have demonstrated that the unsteady aerodynamic phenomena of the flapping mechanism are not only sensitive to variations in the wing kinematics but also to the wing morphing [34,35]. Results also revealed that a dynamically adjustable wing surface may potentially provide new aerodynamic mechanisms $[36,37]$ of force production over fully rigid wings $[38,39]$ in flapping flight. Further research $[40,41]$ has illustrated that the performance of a rigid flapping wing can be improved significantly by adding some level of flexibility to the wing surface. For achieving the performance enhancement, both passive and active flow control mechanisms have been studied, and some examples are reviewed here.

In order to understand the effects of chord-wise flexibility on aerodynamics of flapping motion, a hinge connected two rigid components model is commonly used because of its simplicity and well preservation of the flexibility characteristics in chord-wise [42]. Through this passive deformable model, Eldredge et al. [43] revealed that wings with moderate flexibility have better power efficiency compared to the rigid wing in hovering flight, nevertheless very flexible wings will degrade its aerodynamic performance. Using a similar model, Vanella et al. [44] showed that if parameters are chosen appropriately, the chord-wise flexibility can result in an enhancement of up to $28 \%$ in the lift-to-drag ratio and a $39 \%$ increase in the lift-to-power ratio over a rigid plate. Wan et al. [45] studied the effect of chord-wise flexibility over a range of hovering kinematic parameters using a hinged-plate model. Their results indicated that the maximum lift-to-drag ratio can be achieved by placing the hinge at the three-quarter chord position from the leading edge. 
In addition to above passive mechanisms explorations, active flow control via wing surface morphing has been widely adopted in both fixed-wing and rotary-wing aircraft design. These include articulated flaps and/or slats [46], surface flow control devices [47] and continuously deforming surfaces [48]. Among them, the flow control via trailing-edge flap (TEF) is presumed to be more applicable to novel flapping-wing MAVs designs, in terms of simplicity of moving surface control, weight of MAVs and so on. Liu et al. [49] experimentally studied the effect of actively controlled trailing-edge flaps on the flow control of translational plates. Their results have shown that force and flow characteristics strongly depend on the timing of trailing-edge flap deflection rather than translational speed. Li et al. [15] and Xu et al. [14] further studied the effect of the trailing-edge flap on the aerodynamic performance of a 2-D flapping wing via a computational approach. It is found that the aerodynamic forces can be significantly affected by controlling the timing of the flap deflection in a flapping cycle.

\subsection{Force Enhancement Mechanism in Tandem Flapping Wings}

The above-mentioned unsteady force-generation mechanisms occur in a single flapping wing. When tandem wings flap together, one wing will interact with the wake generated by the other. This flow phenomenon can be observed in quad-winged natural fliers. Unlike most other insects such as flies, wasps, and cicadas, which have either reduced their hindwings or mechanically coupled their forewings and hindwings, dragonflies have maintained two independently controlled pairs of wings throughout their evolution [50]. Their neuromuscular system allows them to change many aspects of motion in a single wing actively, including the angle of attack, stroke amplitude, and stroke plane. This gives them the unique capability of controlling flight. In addition, their forewings and hindwings have different shapes and 
mechanical properties. The hindwing is wider, especially the portion close to the wing root. The value of the elastic modulus and hardness of the forewing are greater than that of the hindwing [51]. These distinct features have attracted researchers' attention for a long period to study the aerodynamics of this special quad-winged insect through a variety of numerical and experimental methods.

Lan and Sun [52] conducted 2-D computational studies of two tandem airfoils in flapping motions at a Reynolds number of 1000. As the two airfoils moved parallel, large force enhancement can be achieved by placing two foils with smaller spacing and locating the hindairfoil in a lower vertical position than the fore-airfoil. As an extension work [53], they further explored the effect of phase difference and showed that in-phase flapping could produce the largest vertical force while a $90^{\circ}$ phase difference would result in resultant force reduction. Wang and Russell [54] filmed the wing kinematics of a tethered dragonfly and then numerically studies the 2-D projection of a 3-D wing kinematics at two-thirds the span. Their simulations revealed that the forewing-hindwing phase variation can significantly affect the force generation and power consumption up to $60 \%$ and $40 \%$, respectively. To achieve steady hovering with minimum power, the phase difference should vary between $100^{\circ}$ and $220^{\circ}$. This phase relation results in a typical hindwing leads motion, which agrees well with previous experimental measurements of dragonfly free flight [55]. Hsieh et al. [56] used 2-D force decomposition to examine the tandem flapping wings configuration at the low Reynolds number 625 . Their results indicated that the surface vorticity contribution to the total force is not negligible under such a low Reynolds number, and the volume-vorticity elements play exclusively important roles in revealing mechanisms of aerodynamic forces. Broering et al. [57] carried out 2-D numerical simulations on a tandem-wing flapping configuration at a Reynolds number of $10^{4}$ and Strouhal 
number of 0.3 . The tandem wings were found to produce high thrust at $0^{\circ}$ phase lag, and to achieve high power efficiency at $90^{\circ}$ and $180^{\circ}$ due to the hindwing extracting energy from the wake of the forewing. Along with these 2-D studies, 3-D numerical simulations have also been conducted. Sun and Lan [58] utilized the wing kinematics model presented by Norberg [59,60] to simulate the 3-D hovering flight of dragonfly at the Reynolds number of 1350 . Their simulation results showed that the large stroke plane angle made drag force become the major source for supporting the body weight. The drag and lift contributed to the total vertical force about $65 \%$ and $35 \%$, respectively. Also, the forewing-hindwing interaction effect was small and leaded to the vertical force reduction about $14-16 \%$ compared to two wings flapping in isolation. Their further investigation of the aerodynamics of a model dragonfly in forward flight showed that this force reduction gradually decrease as the increasement of the advance ratio [61].

In addition to these computational studies, there are also many experimental investigations on the aerodynamics of tandem flapping wings configuration. Maybury and Lehmann [62] used a 3-D robotic dragonfly model to study the effects on the wing lift force due to modulation of forewing and hindwing phase lag at intermediate Reynolds number ( $R e=1000 \sim 2000)$. By varying the phase relationship between forewing and hindwing, the authors found that the performance of the forewing remained approximately constant while the lift production of hindwing was modulated in an order of two. Their further flow visualization results indicated that this lift modulation can be related to the leading-edge vortex destruction and the orientation of the local flow vector. Usherwood and Lehmann [63] then investigated the aerodynamic efficiency by varying the phasing of robotic dragonfly wings. Their results showed that two-pair wings configuration can improve aerodynamic efficiency, and this is achieved by recovering the wasted energy in the wake. The further measurement of power consumption showed that the forewing- 
hindwing interaction can save $22 \%$ of aerodynamic power expenditure [64]. Rival et al. [65] experimentally measured the force evolution and associated vortex dynamics of a tandem pitching-plunging configuration at $\operatorname{Re}=3000$. They found that the force and vortex development on the fore-plate was unaffected by the tandem configuration while the vortex interaction on the hind-plate was responsible for the variations in performance. Zheng et al. [66] investigated the aerodynamic forces, power consumption and efficiencies of flexible tandem wings in a water tunnel at a Reynolds number of 3873 . Three wing models with different effective stiffness are tested and compared with the performance of rigid wings in both hovering and forward flight conditions. The force measurements showed that wing model with highest effective stiffness can achieve largest total horizontal force coefficient and efficiency. Their particle image velocimetry (PIV) and wing deformation measurements revealed that the spanwise bending deformation of the wing model can improve the LEV attachment in both hovering and forward flights.

\subsection{Current Objectives}

Despite significant progress have been achieved in the field of computational modeling of flapping wings, there are several aspects remain unclear. While some of these are related to the effects of dynamic surface morphing on the flow modulation, others are more fundamental in terms of flow development of low-aspect-ratio flapping wings. Specifically, the current study will set out to answer the following questions:

1. What are the dominant parameters needed to control the dynamic deformation of flapping wings to achieve high aerodynamic performance? 
2. What are the general features of wake pattern generated by low-aspect-ratio flapping wings, and how are they different from previously observed 2-D and quasi-3D wake topologies?

3. How does the surface morphology and its relative aerodynamic functions in real insect flight be quantified?

4. How do flow interactions between tandem flapping wings enhance aerodynamic performance?

These above questions will be explored using both simplified canonical models (the first two objectives) and high-fidelity modeling of real insects (the second two objectives). The aerodynamic performance and vortex formation of the flapping wing models will be explored via computational fluid dynamics simulations. Numerical simulations are carried out using an inhouse immersed-boundary-method-based direct numerical simulation (DNS) solver. To better understand flow data, leading-edge vortex strength, wing surface pressure distribution, and wake structures will be visualized and analyzed in detail. The primary goals of the current analyses are to (1) offer an improved understanding of the flow physics of bio-inspired propulsion, and (2) offer insights for the design of the next generation of micro air vehicles.

\section{Objective 1: Study the effects of dynamic surface deformation in flapping motion}

To examine the effects of wing morphing on unsteady aerodynamics, deformable flapping plates are numerically studied at low-Reynolds-numbers. The chord-wise camber is modeled by a hinge connecting two rigid components. The leading portion is driven by a biological hovering motion along a horizontal stroke plane. The hinged trailing-edge flap (TEF) is controlled by a 
prescribed harmonic deflection motion. A systematic parametric study investigates the effects of TEF deflection amplitude, deflection phase difference, hinge location, and Reynolds numbers on the near-field and far-field flow structures and their associated aerodynamic performance. Both instantaneous and cycle-averaged forces productions are examined to provide quantitative descriptions of the TEF effects. Furthermore, 3-D effects are also evaluated using a low aspectratio $(A R=2)$ deformable plate.

\section{Objective 2: Investigate the wake topology of low-aspect-ratio flapping wings}

The wake topology and propulsive performance of low-aspect-ratio plates undergoing a pitching-rolling motion in a uniform stream are numerically investigated. The pitching-rolling plate can be treated as a simplified model for mimicking the flapping motion of insect wings and fish pectoral fins. This study investigates the effects of variation of dominant parameters that govern wake structure and thrust performance, including Strouhal numbers, Reynolds numbers, aspect ratios, and phase difference between pitching and rolling motion. The flow features observed in the current study will be compared with previous reported 2-D and quasi-3D wake topologies of flapping foils. In addition, the proper orthogonal decomposition (POD) based force survey method (FSM) is used to reveal the connection between POD modes and their contributions to force generation.

\section{Objective 3: Examine aerodynamics functions of surface morphing in forward flight}

In this study, a high-speed photogrammetry system, 3-D surface reconstruction technology and numerical simulations are used to reveal the effects of morphing wings of a cruising dragonfly. Specifically, the flapping morphing wing kinematics of a free-flight dragonfly are 
measured and quantified first. We then use the reconstructed model to explore the effects of morphing wings, first by removing camber while keeping the same time-varying twist distribution, and second by removing both the camber and the spanwise twist. Numerical simulations are carried out using an in-house immersed-boundary-method-based direct numerical simulation (DNS) solver. To get a better understanding of the aerodynamic roles of morphing wings, the leading-edge vortex, the wing surface pressure distribution, and wake structures are analyzed and compared in detail for the model wings.

\section{Objective 4: Explore the aerodynamic functions of quad-winged flyer in turning maneuver}

Dragonflies are known for their impressive flight agility and maneuverability. Distinct from other insects, dragonflies can move their two pairs of wings independently to achieve their flight control. Most previous studies have mainly focused on the steady flight of dragonflies, and there is a lack of sufficient information regarding wing kinematics and its associated aerodynamics on unsteady motions. To fill this gap, an integrated study combining high-speed photogrammetry, 3D surface reconstruction and direct numerical simulation are conducted to study dragonflies in turning maneuvers. Results of the present work aim to provide insights into future agile quadwinged micro air vehicle (MAV) designs and applications.

\subsection{Outline of Thesis}

To investigate the wake topology and propulsive performance of flapping wings, results will be presented using a combination of simplified canonical model problems and high-fidelity models of real insects. The remainder of the thesis is organized as follows: 
Chapter 2 describes details of numerical method and high-fidelity surface reconstruction technique applied in the current study. In addition, the proper orthogonal decomposition (POD) and force survey method (FSM) are also introduced.

Chapter 3 discusses the computational results of deformable flapping wings hinged with a trailing-edge flap. Section 3.1 and 3.2 describe the problem formulation and computational setup, respectively. Section 3.3 presents the simulations results and analysis on aerodynamic performance and vortical structures. Finally, a brief chapter summary is given in Section 3.4. The results of Chapter 3 form the basis of the following publications:

- Chengyu Li, Haibo Dong, and Geng Liu, "Effects of a dynamic trailing-edge flap on the aerodynamic performance and flow structures in hovering flight," Journal of Fluids and Structures 58, 49-65 (2015).

- Min Xu, Mingjun Wei, Chengyu Li, and Haibo Dong, "Adjoint-based optimization of flapping plates hinged with a trailing-edge flap," Theoretical \& Applied Mechanics Letters 5, 1-4 (2015).

- $\quad$ Chengyu Li, Haibo Dong, and Yan Ren, "A Numerical Study of Flapping Plates Hinged with a Trailing-Edge Flap," 32 ${ }^{\text {nd }}$ AIAA Applied Aerodynamics Conference, Atlanta, Georgia, June 2014.

Chapter 4 presents the computational results of wake topology and propulsive performance of low-aspect-ratio pitching-rolling plates over a range of Strouhal numbers, Reynolds numbers, aspect-ratios, and phase difference angles. The Chapter is begins by describing problem definition and computational setup in Section 4.1 and Section 4.2, respectively. This is followed with a detailed discussion of wake topology and propulsive performance in Section 4.3. In 
Section 4.4, a POD-based flow analysis is conducted to reveal the relationship between POD modes and force generation. Finally, the conclusions are given in Section 4.5. The results of Chapter 4 form the basis of the following publications:

- Chengyu Li and Haibo Dong, "Three-dimensional wake topology and propulsive performance of low-aspect-ratio pitching-rolling plates," Physics of Fluids (2016), under revision.

- Chengyu Li and Haibo Dong, "Quantification and Analysis of Propulsive Wake Topologies in Finite Aspect-Ratio Pitching-Rolling Plates," $46^{\text {th }}$ AIAA Fluid Dynamics Conference and Exhibit, Washington D.C., June 2016, accepted.

- Chengyu Li, Haibo Dong, and Zongxian Liang, "Proper Orthogonal Decomposition Analysis of 3-D Wake Structures in a Pitching-rolling Plate," 54 $4^{\text {nd }}$ AIAA Aerospace Sciences Meeting,San Diego, California, January 2016.

Chapter 5 presents and discusses the computational results of the flapping morphing wing of a cruising dragonfly. Section 5.1 and 5.2 demonstrate the dragonfly surface reconstruction and computational setup, respectively. It follows with the quantification of wing kinematics and surface deformation of morphing wings (Section 5.3). Section 5.4 presents the aerodynamic performance of flapping wings. Section 5.5 describes the vortex formation in detail. Finally, the conclusions are given in Section 5.6. The results of Chapter 5 form the basis of the following journal publication:

- Chengyu Li and Haibo Dong, "Improvement of Aerodynamic Performance by Surface Deformation of a Dragonfly in Forward Flight," Journal of Royal Society Interface, under preparation. 
Chapter 6 discusses the computational results of a dragonfly's turning maneuver. The dragonfly surface reconstruction and computational setup is shown in Section 6.1 and Section 6.2, respectively. It follows by the quantification of body and wing kinematics in Section 6.3. The aerodynamic performance of flapping wings are presented in Section 6.4. The detail description of vortex formation is given in Section 0. Furthermore, the effect of wing-wing interaction is discussed in Section 6.6. Finally, the conclusions are given in Section 6.7. The results of Chapter 6 form the basis of the following journal publication:

- Chengyu Li and Haibo Dong, "Computational investigation of dragonfly aerodynamics in turning maneuver," Bioinspiration \& Biomimetic, under preparation.

Chapter 7 summarizes the conclusions of the current computational studies and points toward future work. 


\section{Numerical Methodology}

\subsection{Numerical Method}

In the current study, flow fields are generated by a direct numerical simulation of the threedimensional unsteady, viscous incompressible Navier-Stokes equations, as written in the following:

$$
\begin{gathered}
\frac{\partial u_{j}}{\partial x_{j}}=0 \\
\frac{\partial u_{i}}{\partial t}+\frac{\partial\left(u_{i} u_{j}\right)}{\partial x_{j}}=-\frac{1}{\rho} \frac{\partial p}{\partial x_{i}}+v \frac{\partial}{\partial x_{j}}\left(\frac{\partial u_{i}}{\partial x_{j}}\right)
\end{gathered}
$$

where $u_{i}(i=1,2,3)$ are the velocity components in the $\mathrm{x}-, \mathrm{y}$ - and $\mathrm{z}$-directions, respectively; $p$ is the static pressure; $\rho$ and $v$ are the fluid density and kinematic viscosity, respectively.

The equations (Eq. ((2-1) and ((2-2)) are discretized using a second-order central difference scheme on a non-uniform Cartesian mesh, where the velocity $\left(u_{i}\right)$ and pressure $(p)$ are collocated at the cell centers. The unsteady equations are solved using a fractional step method [67], which provides second-order accuracy in time. An Adams-Bashforth scheme and an implicit Crank-Nicolson scheme are employed for the discretization of the convective terms and diffusion terms, respectively. Briefly, the governing equations (Eq. ((2-1) and ((2-2)) are solved by three sub-steps. In the first step, the solution is advanced by solving the advection-diffusion equation, as shown in Eq. (2-3), to obtain an intermediate velocity filed $\left(u_{i}^{*}\right)$. Subsequently, a Modified Strongly Implicit Procedure (MSIP) method [68] is employed to solve the pressure Poisson equation (Eq. (2-4)) to obtain the pressure correction $\left(p^{\prime}\right)$. Finally, the cell-centered velocity $\left(u_{i}\right)$ and face-centered velocity $\left(U_{i}\right)$ are updated using the corrected pressure, respectively, as presented in Eq. (2-5) and (2-6). 


$$
\begin{gathered}
\frac{u_{i}^{*}-u_{i}^{n}}{\Delta t}=\frac{1}{2}\left(-\frac{\delta\left(U_{j} u_{i}\right)}{\delta x_{j}}+v \frac{\delta}{\delta x_{j}} \frac{\delta u_{i}}{\delta x_{j}}\right)^{*}+\frac{1}{2}\left(-\frac{\delta\left(U_{j} u_{i}\right)}{\delta x_{j}}+v \frac{\delta}{\delta x_{j}} \frac{\delta u_{i}}{\delta x_{j}}\right)^{n} \\
\frac{1}{\rho} \frac{\delta}{\delta x_{j}} \frac{\delta p^{\prime}}{\delta x_{j}}=-\frac{1}{\Delta t}\left(\frac{\delta U_{i}^{*}}{\delta x_{i}}\right) \\
u_{i}^{n+1}=u_{i}^{*}-\frac{\Delta t}{\rho}\left(\frac{\delta p^{\prime}}{\delta x_{j}}\right)_{C C} \\
U_{i}^{n+1}=U_{i}^{*}-\frac{\Delta t}{\rho}\left(\frac{\delta p^{\prime}}{\delta x_{j}}\right)_{F C}
\end{gathered}
$$

Where, $\delta$ is the central difference operator; CC and FS represent the update of velocity filed based on cell-centered and face-centered, respectively.

The flow solver employs a sharp-interface immersed boundary method (IBM) based on the multi-dimensional ghost-cell methodology as described in Ref. [26,69]. The IBM is an innovative approach in simulating fluid flow over bodies which are imbedded within a Cartesian grid. The general process begins by generating the immersed boundary comprised of a number of densely spaced marker points connected by linear segments. A non-conformal Cartesian grid is then generated followed by a procedure that identifies the fluid-cells and solid-cells. Fluid-cells are cells whose cell centers line outside of the body, and solid-cells are made up of the remaining cells with cell centers inside the body that are not adjacent to the immersed boundary.

The key concept of the immersed boundary method is to compute the flow variables for the ghost-cells, such that boundary conditions on the immersed boundary in the vicinity of the ghostcells are satisfied while preserving second-order accuracy. Ghost-cells are those cells whose 
centers lie inside the immersed body and have at least one neighboring cell which lies outside the immersed body. As shown in Figure 2-1, the boundary condition on the body-intercept point is satisfied by imposing the value on the ghost cell. The value at the ghost cell is obtained from the image point which is computed by the interpolation of the values on the surrounding fluid cells. Thus, flow simulations with complex boundaries are conducted on stationary non-bodyconformal Cartesian grids. This arrangement eliminates the need for the complicated re-meshing algorithms that are usually employed by conventional Lagrangian body-conformal methods. Additional details for this code can be found in Ref. [69]. The current flow solver has been used to model translational/rotational plates [70,71], pitching-rolling plates [72], and the flapping wing of a cicada [73]. The validations of the current solver are provided in Appendix A.

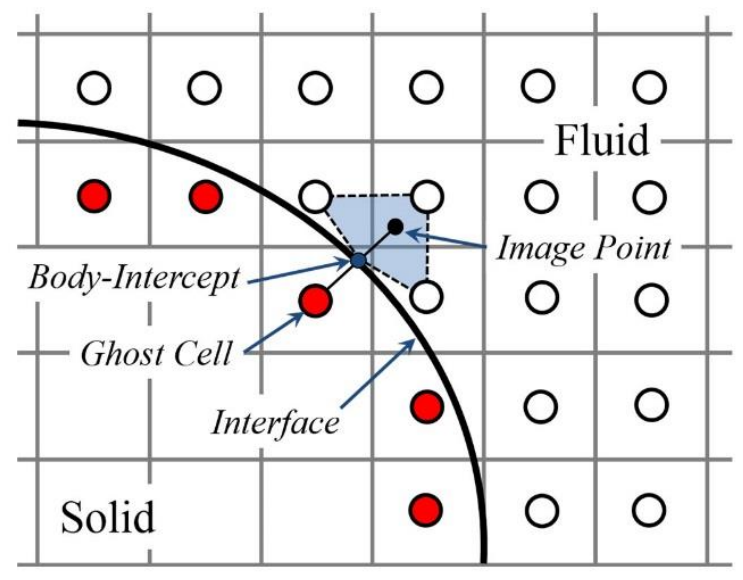

Figure 2-1. Schematic of the ghost cell method for the immersed boundary treatment.

\subsection{High-Speed Photogrammetry and Surface Reconstruction}

Image sequences of free flying insects are collected using three synchronized Photron Fastcam SA3 60K high-speed cameras capable of up to 1000 frames per second at a resolution of $1024 \times 1024$ pixels resolution with a global shutter speed of 2 us. Three cameras are fixed on an aluminum framework, as shown in Figure 2-2. The framework and foundation can ensure that 
cameras are aligned orthogonal to each other on an optical breadboard. The slotted channels in framework allow us to adjust the distance between cameras and the insects. For providing excellent temporal and spatial resolution, the cameras are positioned 1.5 meters away from the dragonfly based on the body size and flapping frequency of the specimen. The optical breadboard not only allows us to mount our hardware to a sturdy anchor, but also minimizes vibrations will occur within the system. For the lighting system, two halogen photo optic lamps (OSRAM, 54428) are chose for our experiment.

(a)

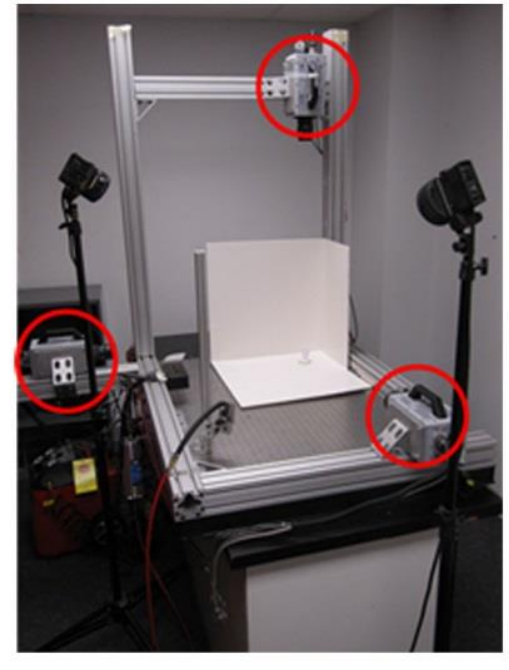

(b)

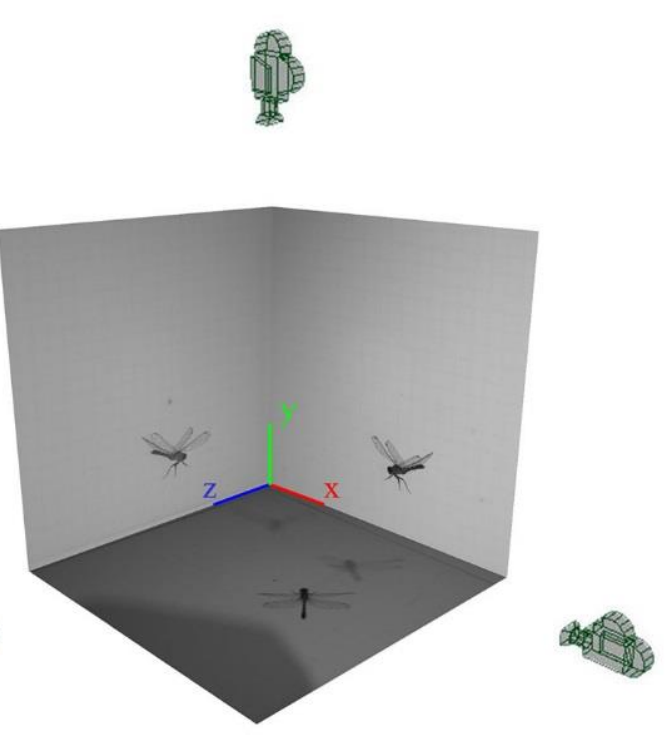

Figure 2-2. (a) High-speed camera system set-up in the lab. (b) Conceptual model of the photogrammetry system used to capture insects in free flight based on Autodesk Maya.

For reconstructing the wing kinematics and deformation, each insect wing is marked with a fine tipped permanent marker before shot videos. Since the added weight from the ink on the surface of the wing is pretty small, we assume it is approximated as zero and does not affect the flight performance. For an arbitrary point on a dragonfly's wing in each frame, we use the 
perspective projections method to decide its location in multiple projection planes. The photogrammetry system is used to capture dragonfly in flight.
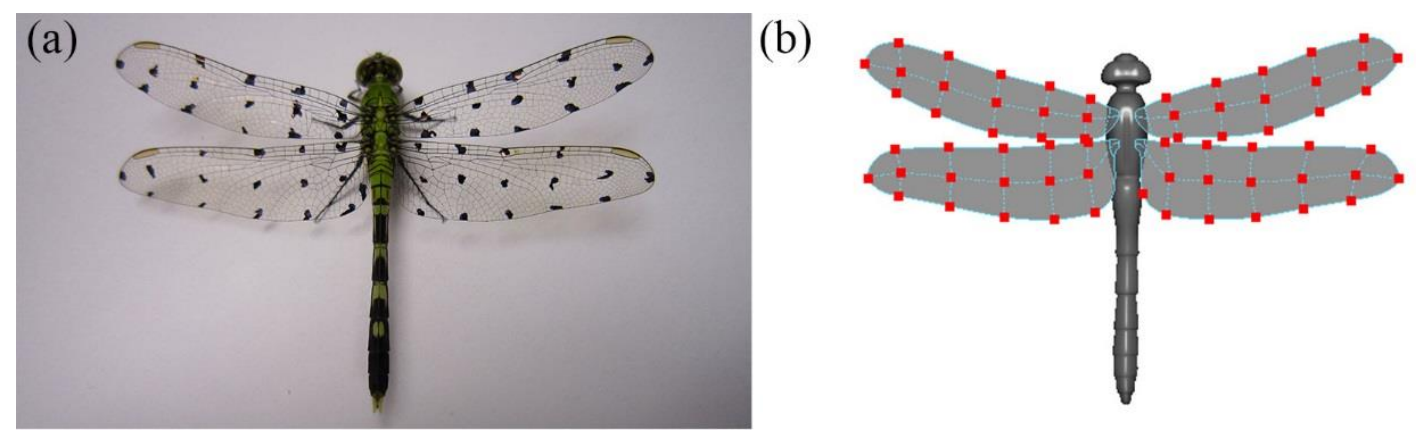

Figure 2-3. Initial configuration of a dragonfly template mesh. (a) Top-down image is showing the marker points on the wings. (b) Wing and body template models with the surface points corresponding to the top level of the subdivision hierarchy marked in red.

The initial 3-D wing template models are generated with Catmull-Clark subdivision surfaces by using a computer graphics software Autodesk Maya (as shown in Figure 2-3). Based on the high speed films, we align first level vertices of the subdivision surface hierarchy corresponding to the marker points on dragonfly's fore-wings and hind-wings. After the initial template surfaces of wings are generated, they are recorded as a key frame animation. By repeatedly adjusting the anchor point based alignment process along with each axis for each time step, the first level vertices of wings are completed. Even the whole process of wing reconstruction is a little bit labor intensive, but it is currently the only efficient way to reconstruct a deformable, quad-winged insect in free-flight (as shown in Figure 2-4). Thus, the approximation of the 3-D wing shapes such as span-wise bend, chord-wise bend, and twist can be captured with smooth subdivision surface representation. Comparing with tethered insects, free-flying insects present many challenges to the surface reconstruction work due to those non- 
linear translation and rotation motion, especially for dealing with maneuver cases. Analysis of the projection errors for filming geometry shows that the reconstructed angles is less than 0.5 degree in error over the entire filming area and that the assumed horizontal plane deviates by less than 1 degree from the true horizontal. Perspective errors can thus be ignored during the kinematic analysis.
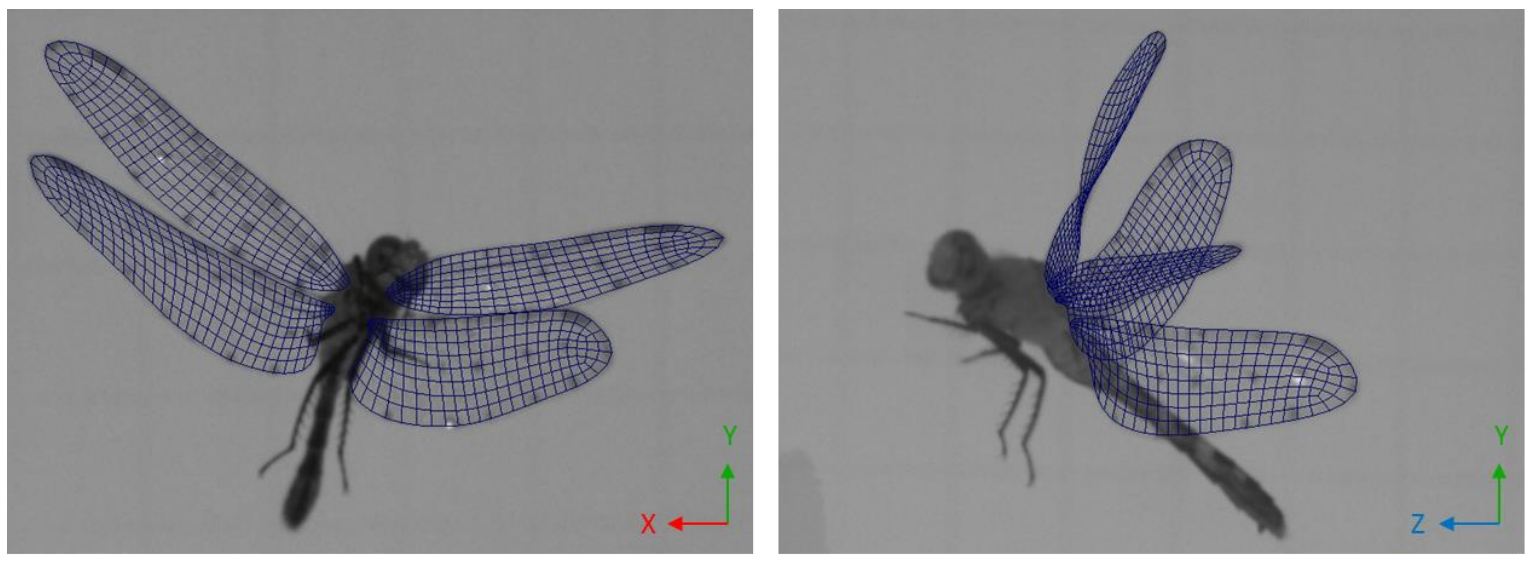

Figure 2-4. Reconstructed wings at a time step where a large amount of twist and camber is present in multiple wings.

\subsection{Proper Orthogonal Decomposition (POD)}

Proper Orthogonal Decomposition (POD) is a method used to represent large data fields with a relatively small number of elements. POD creates a set of basis function which spans the original data set by capturing the characteristic components. The system can be represented by the first few dominant modes. In the POD analysis of flow field, velocity vectors are written as an ensemble of spatial and temporal components: 


$$
\mathrm{u}(\mathrm{x}, t)=\overline{\mathrm{u}}+\sum_{i=1}^{N_{m}} \alpha_{i}(t) \Phi_{i}(\mathrm{x})
$$

where $\overline{\mathrm{u}}$ is mean velocity, $\Phi_{i}$ are POD modes, $\alpha_{i}$ are temporal coefficients of the modes $\Phi_{i}$, and $N_{m}$ is total number of modes.

To form a set of the modes $\Phi_{i}$ in the context of proper orthogonal decomposition, it is required to solve an optimization problem that maximizes the quantity $\left\langle\left(u^{\prime}, \Phi\right)^{2}\right\rangle$ subjected to a constraint $\|\Phi\|=1$ for each mode $\Phi_{i}$ in the set. The POD method in this scenario produces the POD modes $\Phi_{i}$ that maximize the resolution of fluctuating kinetic energy. The optimization problem can be transformed into an eigenvalue problem by using the snapshot method [74], written as $\mathrm{AV}=\lambda \mathrm{V}$, in which the size of the correlation matrix $\mathrm{A}$ is $N_{S} \times N_{S}$, and the entries of matrix $\mathrm{A}$ are $a_{i j}=\left(\mathrm{u}_{i}^{\prime}, \mathrm{u}_{j}^{\prime}\right) . N_{S}$ is the number of snapshots. Solving the eigenvalue problem yield eigenvalues and eigenvectors for constructing the modes $\Phi_{i}$. Given the POD modes $\Phi_{i}$, the $n$th snapshot $\mathrm{u}^{n}$ can be recovered from a linear combination of the POD modes:

$$
\mathrm{u}^{n}=\overline{\mathrm{u}}+\sum_{i=1}^{N_{m}} \alpha_{i}^{n} \Phi_{i}
$$

where the temporal coefficients $\alpha_{i}^{n}=\left(\Phi_{i}, \mathrm{u}_{n}^{\prime}\right)$. 


\subsection{Virtual Force of POD Modes}

Based on POD mode force survey method (POD-FSM), the force acting on an immersed body can be written in terms of a linear combination of non-interaction and interaction force terms:

$$
\mathrm{F}=\sum_{i=0}^{N_{m}} \mathrm{~F}_{i}+\sum_{j=0}^{N_{m}} \sum_{i=0}^{N_{m}} \mathrm{~F}_{i j}
$$

where $\mathrm{F}_{i}$ are the virtual forces caused by the $i$ th POD mode and $\mathrm{F}_{i j}$ are the virtual forces caused by the interaction between the $i$ th and $j$ th POD modes. The forces related to POD modes are termed 'virtual forces' in the current paper.

The non-interaction term, $\mathrm{F}_{i}$, are consisting of one volume integral term related to the first moment of POD vorticity and three surface integral terms related to the viscous stress tensor. The interaction terms $\mathrm{F}_{i j}$ computer the interaction of the $i$ th and $j$ th POD mode at the external surface $S$. The volume and surface integrals in $\mathrm{F}_{i}$ and $\mathrm{F}_{i j}$ are functions of POD modes and are independent of time. However, $\mathrm{F}_{i}$ and $\mathrm{F}_{i j}$ are scalar functions of temporal coefficients. Therefore, it can be concluded that the force direction and magnitude are determined by POD modes and temporal coefficients, respectively. More detail information and validations of the POD-FSM can be found in Ref. [75]. 


\section{Effects of a Dynamic Trailing-Edge Flap in Flapping Motion}

Vein-reinforced membrane structure can be widely observed in insect wings. During flapping, the wing will deform around certain pivot points and form camber deformation along its chordwise direction (Figure 3-1). Inspired by the previous morphing wing studies, we designed a simplified 2-D model to investigate the time-varying camber effects. The current study aims to find out the dominant parameters of the camber formation to achieve the flow control and improve the aerodynamic performances.

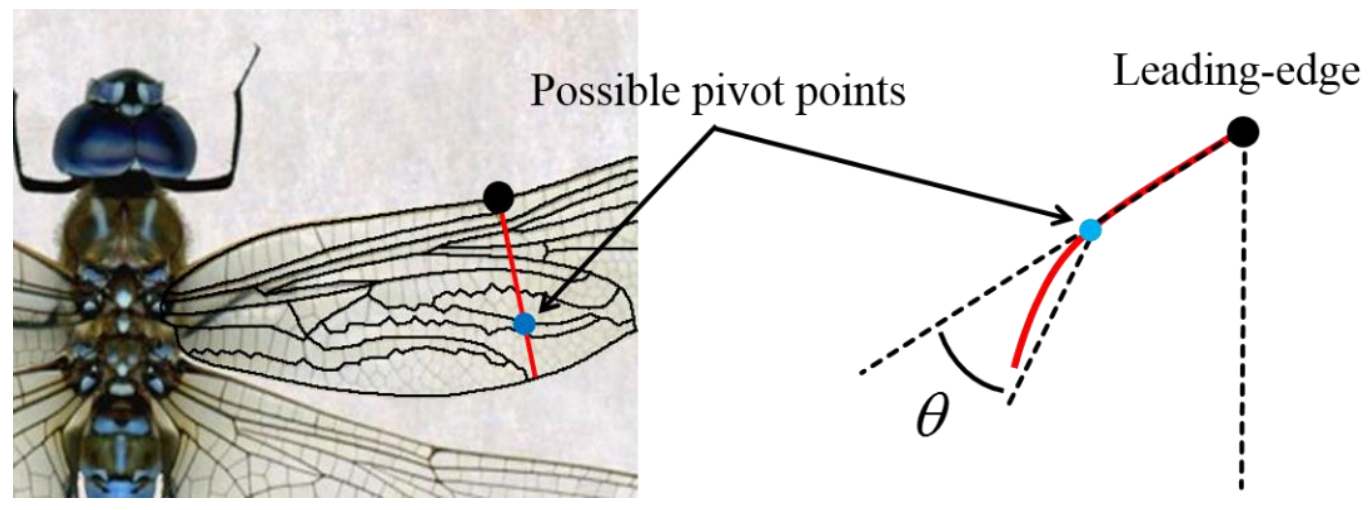

Figure 3-1. Schematics of a vein-stiffened wing and the idolized model for presenting the chordwise deflection $(\theta)$ around a possible pivot point.

\subsection{Problem Definition}

In the present work, a thin rigid plate attached with a TEF is considered as the model of deformable flapping wing, as shown in Figure 3-2 (a). The methodology of handling this thin (0.04 chord-length thickness) plate is demonstrated in Ref.[69]. As shown in Figure 3-2 (b), the harmonic kinematics is used to prescribe the flapping motion in a Cartesian coordinates system. Specifically, the main plate is constrained to move in a horizontal stroke plane according to the function defined by Eq. (3-1) and (3-2): 


$$
\begin{gathered}
x(t)=\frac{A_{0}}{2} \cos (2 \pi f t), \quad \mathrm{y}(t)=0 \\
\theta_{L}(t)=\beta_{L} \sin (2 \pi f t)
\end{gathered}
$$

where, $x(t)$ and $y(t)$ denote the position of the leading edge of the chord, $\theta_{L}(t)$ is the orientation of the main plate relative to the vertical direction, $f$ is the flapping frequency, $A_{0}$ and $\beta_{L}$ are the amplitudes of translation and rotation, respectively.

The deflection angle $\left(\theta_{T}\right)$ of TEF is defined by Eq. (3-3). In the current study, the deflection phase difference $(\varphi)$ controls the TEF deflection timing, and thus forms a different camber pattern. The choices of these design parameters are in line with other TEF studies on fixed/rotary wing aerodynamics $[76,77,78]$. By changing the deflection phase difference, the plate will either form a positive camber with $\varphi \in\left(-90^{\circ}, 90^{\circ}\right)$, or form a negative camber with $\varphi \in\left(90^{\circ}, 270^{\circ}\right)$ which is presented as the sum of $\left(90^{\circ}, 180^{\circ}\right)$ and $\left(-180^{\circ},-90^{\circ}\right)$ in the following discussions. Figure 3-2 (c-d) present the typical samples of a positive camber configuration at $\beta_{T}=60^{\circ}, \varphi=60^{\circ}$ and, a negative camber configuration at $\beta_{T}=60^{\circ}, \varphi=-120^{\circ}$, respectively.

$$
\theta_{T}(t)=\beta_{T} \sin (-2 \pi f t+\varphi)
$$

where, $\beta_{T}$ is the amplitude of deflection, and $\varphi$ is the phase difference between the main plate rotation and trailing-edge deflection. 
(a)

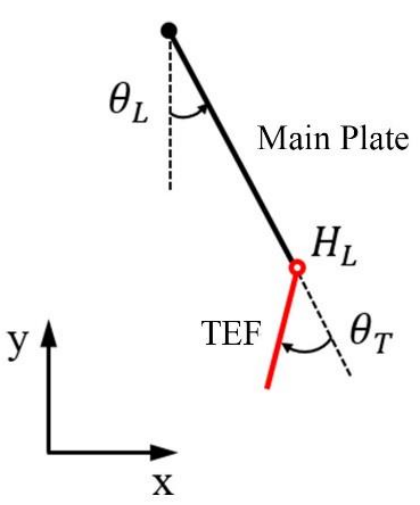

(c)
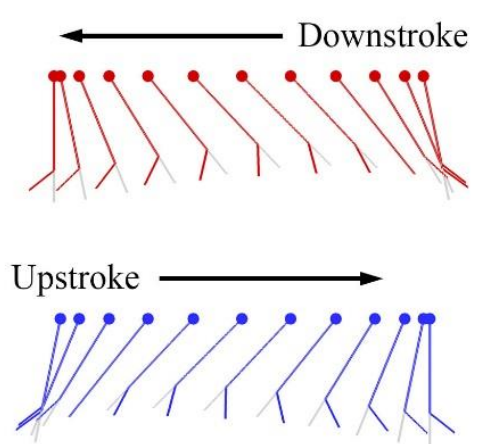

(b)

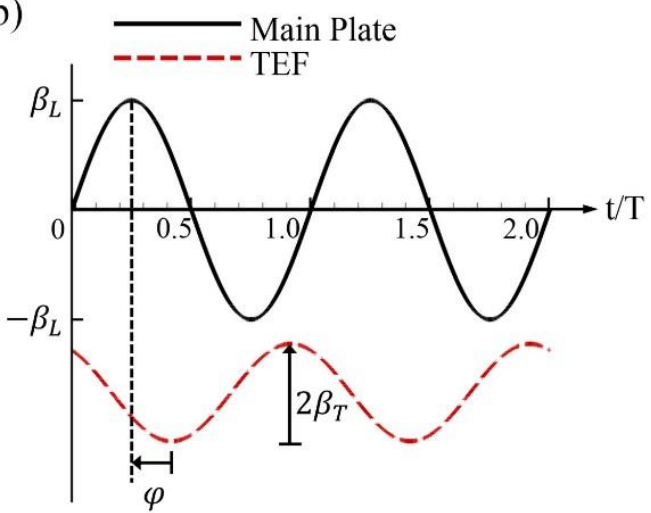

(d)
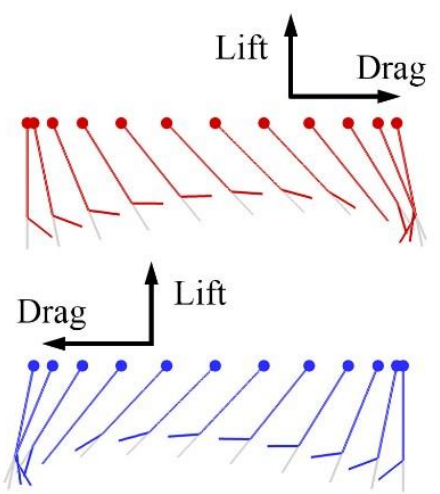

Figure 3-2. (a) A schematic illustration of the specified kinematics parameters. The main plate and TEF are colored in black and red, respectively; (b) Definition of flap deflection amplitude ( $\left.\beta_{T}\right)$ and deflection phase difference $(\varphi)$; (c) Sample of positive camber configuration at $\beta_{T}=60^{\circ}, \varphi=60^{\circ}$; (d) Sample of negative camber configuration at $\beta_{T}=60^{\circ}, \varphi=-120^{\circ}$.

The Reynolds number is defined as $R e=U_{\max } c / v=\pi f A_{0} c / v$, based on the maximum translational velocity $\left(U_{\max }\right)$. The lift and drag coefficients are defined as $C_{L}=L /\left(0.5 \rho U_{\max }^{2} c\right)$ and $C_{D}=D /\left(0.5 \rho U_{\max }^{2} c\right)$, with the lift and drag denoted by $L$ and $D$, respectively. The cycleaveraged lift and drag coefficients are denoted by $\bar{C}_{L}$ and $\bar{C}_{D}$, respectively. The instantaneous 
aerodynamic power is calculated as $P=\sum_{i=1}^{n} \overrightarrow{F_{i}} \cdot \overrightarrow{v_{i}}$, where $n$ is the total number of elements, $\vec{F}_{i}$ is the aerodynamic force acting on each element and $\overrightarrow{v_{i}}$ is the corresponding velocity of the element. The power coefficient can be expressed as $C_{P W}=P /\left(0.5 \rho U_{\max }^{3} c^{2}\right)$. For the calculation of cycleaveraged aerodynamic power $\left(\bar{C}_{P W}\right)$, only the positive power is considered. Further, the lift-topower ratio $\left(\bar{C}_{L} / \bar{C}_{P W}\right)$ is used to evaluate the aerodynamic efficiency. We also noticed that the projected chord length of the flapping plate is time-dependent, and the time-averaged chord length, $\langle c\rangle_{T}$, might be another choice for calculating the force coefficients [79]. It has been found that this may cause slight changes in the force magnitude, however, the overall conclusions of this Chapter remain unaffected.

\subsection{Computational Setup}

Figure 3-3 (a) shows the flapping plate configuration immersed in the 2-D non-uniform Cartesian grid. The coordinate directions, which are also shown in Figure 3-3 (b), are as follows: $\mathrm{x}$ is the horizontal direction with $+\mathrm{x}$ pointing towards right side boundary; and $\mathrm{y}$ is the vertical direction with $+y$ pointing upward. Figure 3-3 (b) shows the boundary conditions applied on the computational domain boundaries. On all of the boundaries, a far-field boundary condition which amounts to specifying the stream-wise (vertical) velocity component to zero and setting the normal gradients of the other velocity components to zero is applied.

The computational domain size of 2-D simulation is $30 c \times 40 c$ with a refined zone of $8 c \times$ $11 c$. The grid $(321 \times 465)$ is chosen with the smallest resolution of $\Delta x=0.02 c$ for the case of $R e=100$, and a finer mesh $(449 \times 673)$ is used for simulations at higher Reynolds number. The 3- 
D simulations are performed in a $30 c \times 30 c \times 30 c$ domain. The grid adopted has $221 \times 265 \times 117$ cells with minimum grid size $\Delta x=0.03 c$. The time-step $(T / d t=960)$ is chosen for the current study corresponds to a Courant-Friedrichs-Lewy (CFL) number around 0.5. We have conducted extensive tests to ensure that the domain is large enough to achieve accurate results, and grid refinement has been performed to make sure that the simulation results are grid-independent in both the 2-D and 3-D cases.

(a)

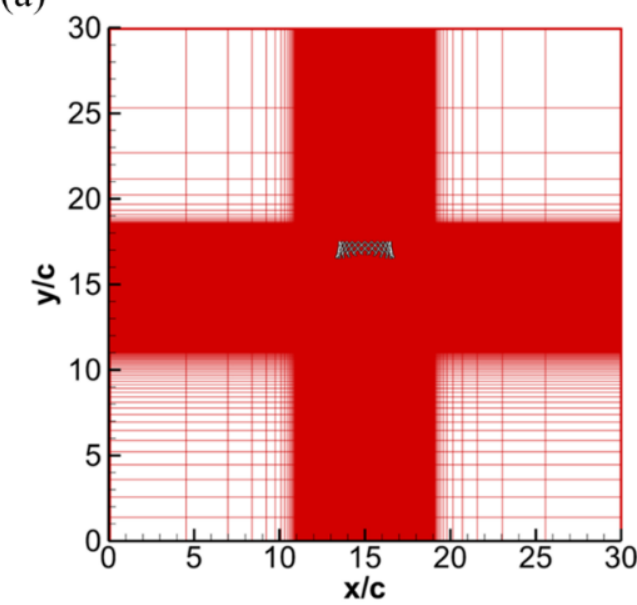

(b)

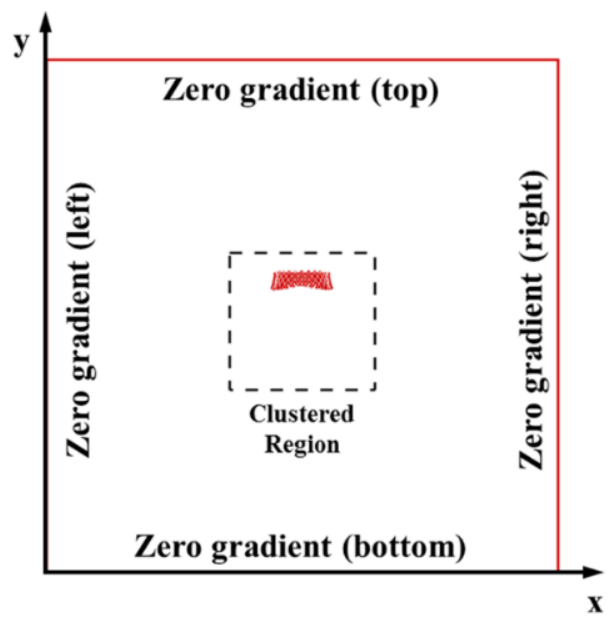

Figure 3-3: (a) Flapping plate configuration immersed in the 2-D non-uniform Cartesian grid.

(b) Boundary conditions for the simulation.

In order to identify a suitable number of grid points and time step, spatial grid and time-step sensitivity analyses are performed for the rigid plate with $A_{0} / c=3.0$. Simulation within fine grid was carried out on a grid which had 52\% more grid points than the medium grid, and simulation within coarse grid was carried out on a mesh with $48 \%$ less grid points than the medium grid. Both of these simulations produced a maximum $1 \%$ difference from the medium grid in mean lift and root-mean-square (r.m.s.) values of lift and drag. The temporal sensitivity study also conducted within the medium grid to guarantee the convergence of current simulation. Each simulation was integrated over 10 flapping cycles. Estimates of cycle mean and r.m.s. lift as well 
as r.m.s. drag indicate that there is a less than a $3 \%$ difference in these quantities from the ninth to the tenth cycle. Thus, the flow quantities reach a near-stationary state by the ninth and tenth cycles and all of the flow statistics in the following discussion are estimated based on averaging over the ninth and tenth cycles. From the results shown in Figure 3-4, the medium grid $\left(0.29 \times 10^{6}\right)$ and $T / d t=960$ are chosen. The time-step chosen for the current study corresponds to a Courant-Friedrichs-Lewy (CFL) number of 0.51 .

(a)

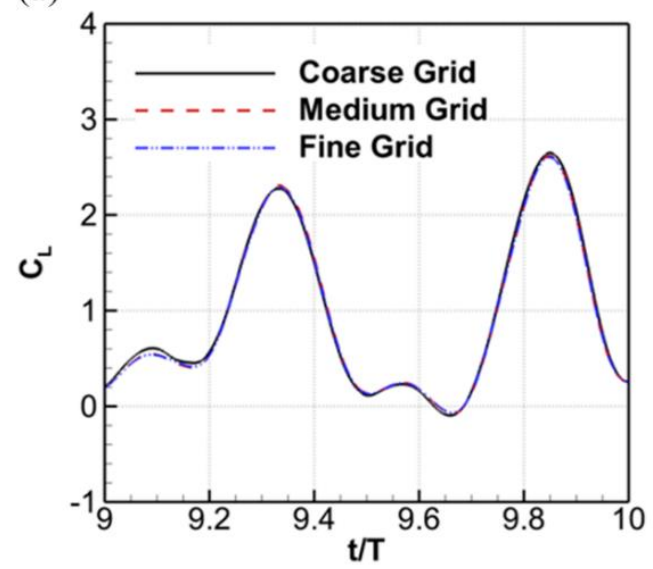

(c)

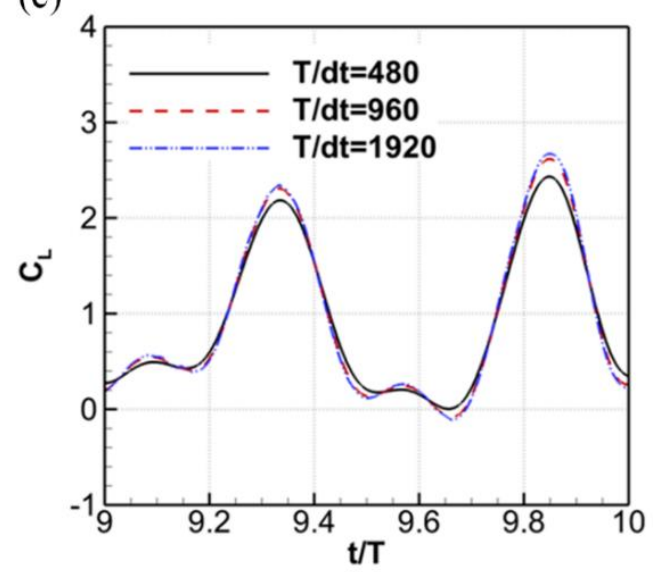

(b)

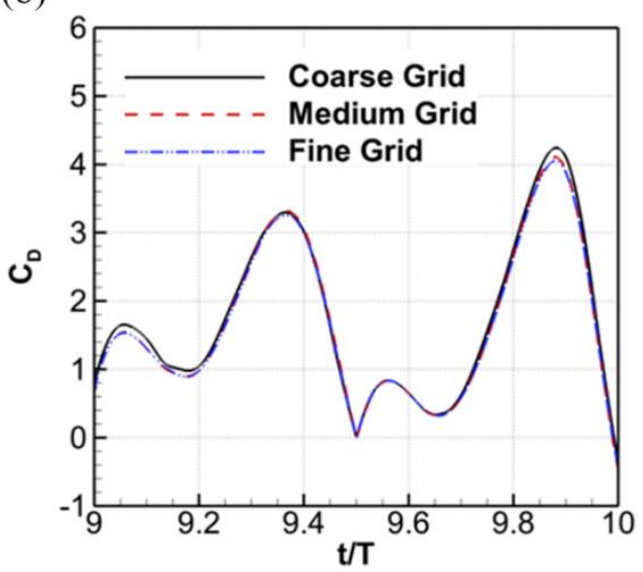

(d)

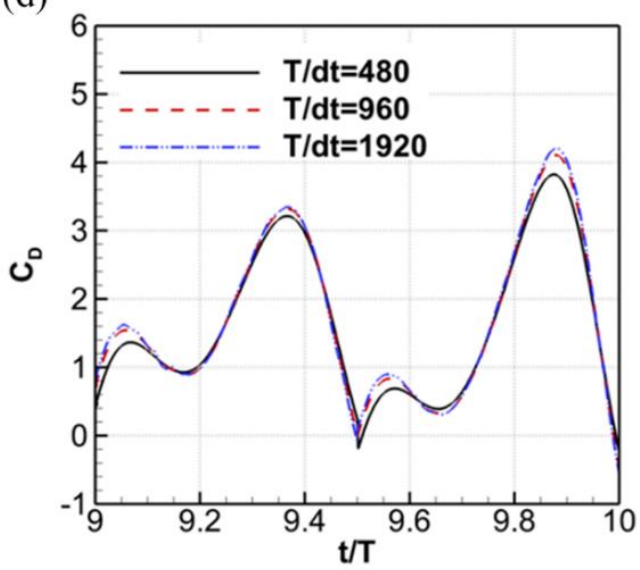

Figure 3-4: Spatial and Temporal sensitivity analyses for a rigid flapping plate at $A_{0} / c=3.0$.

Lift coefficient $(a, c)$ and drag coefficient $(b, d)$ time history for the $10^{\text {th }}$ flapping stroke. 

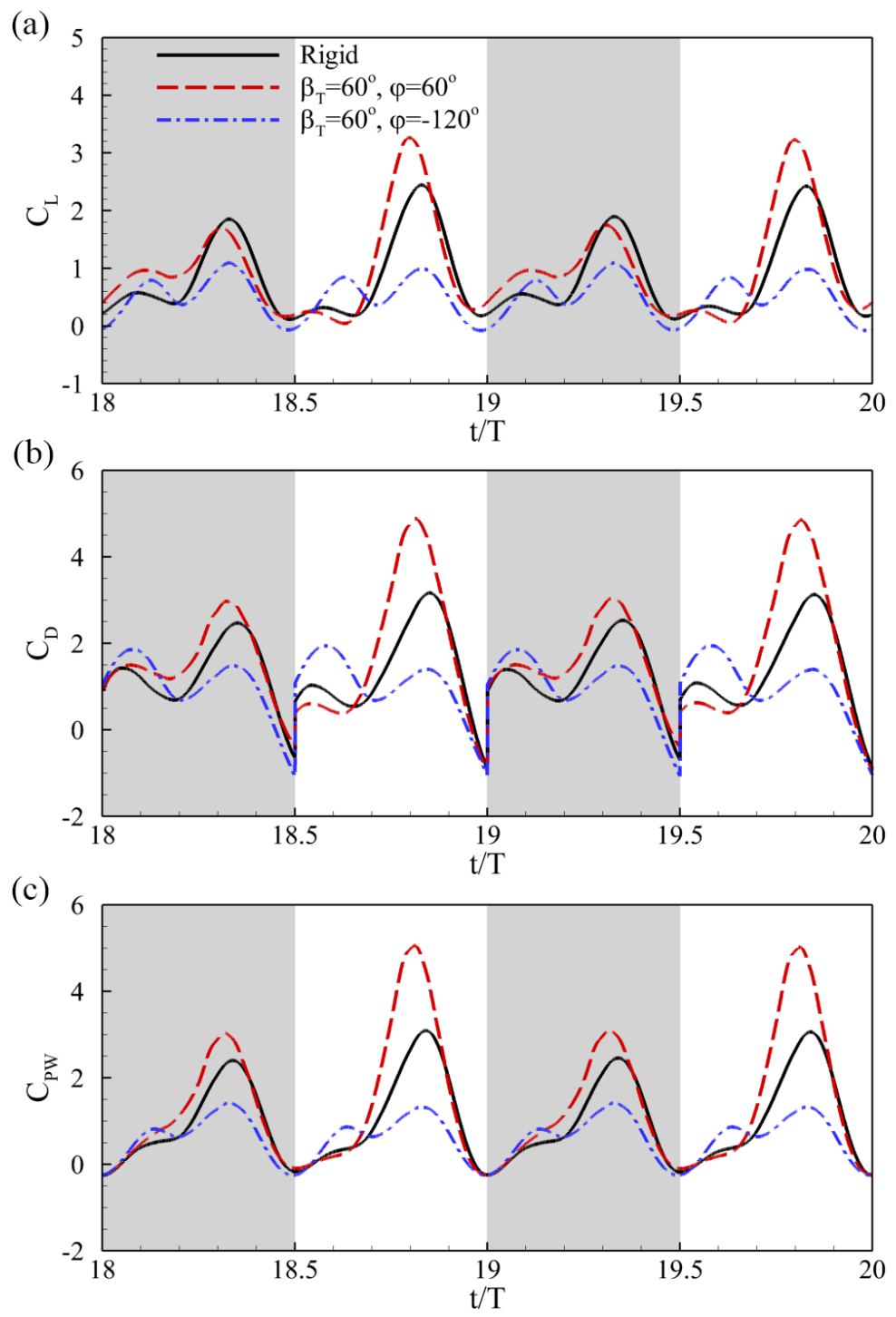

Figure 3-5. Time traces of lift (a), drag (b), and power (c) coefficients for fully rigid plate and plate with a TEF at $\beta_{T}=60^{\circ}, \varphi=60^{\circ}$ (positive camber) and $\beta_{T}=60^{\circ}, \varphi=-120^{\circ}$ (negative camber). The half-cycles representing down-stroke are shaded.

\subsection{Aerodynamic Performance and Vortical Structure}

In this section, we first present the effects of TEF deflection phase difference and deflection amplitude for a hinge location at $H_{L}=0.75 c$, in Section 3.3.1 and 3.3.2, respectively. The effects 
of the hinge location change will then be investigated in Section 3.3.3. In these simulations, the following values are selected for the stroke amplitude, the main plate rotational amplitude, and the Reynolds number: $A_{0} / c=3.0, \beta_{L}=\pi / 4$, and $R e=100$, respectively. The parameters describing the plate kinematics are chosen based on previous work on insect flight $[44,80,81,82]$. The trailing-edge deflection amplitude $\beta_{T}$ ranges from $15^{\circ}$ to $90^{\circ}$, and the deflection phase difference $\varphi$ ranges from $-180^{\circ}$ to $-180^{\circ}$. The effects of the Reynolds number will be examined in Section 3.3.4. Furthermore, low-aspect-ratio 3-D cases will be analyzed by comparing the aerodynamic performance to the 2-D cases in Section 3.3.5. The emphasis of the current study is on the effects of the abovementioned factors on the aerodynamic performance and flow structures.

(a)

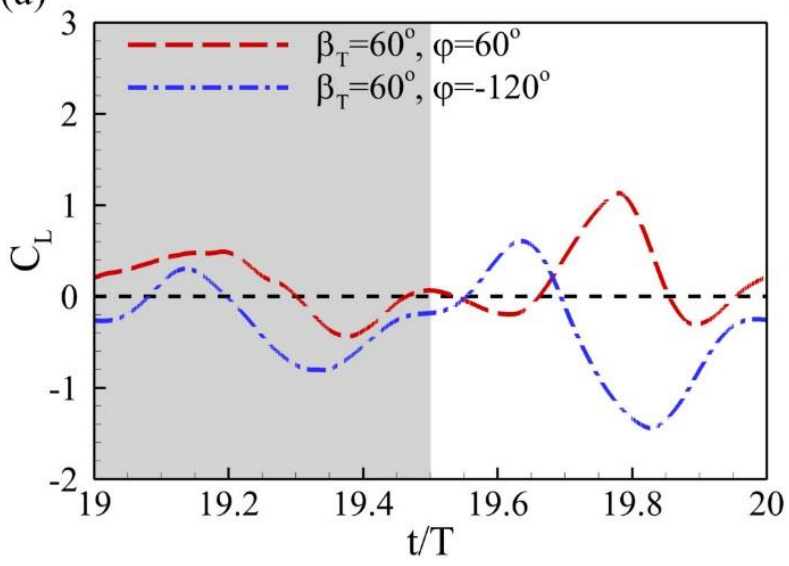

(b)

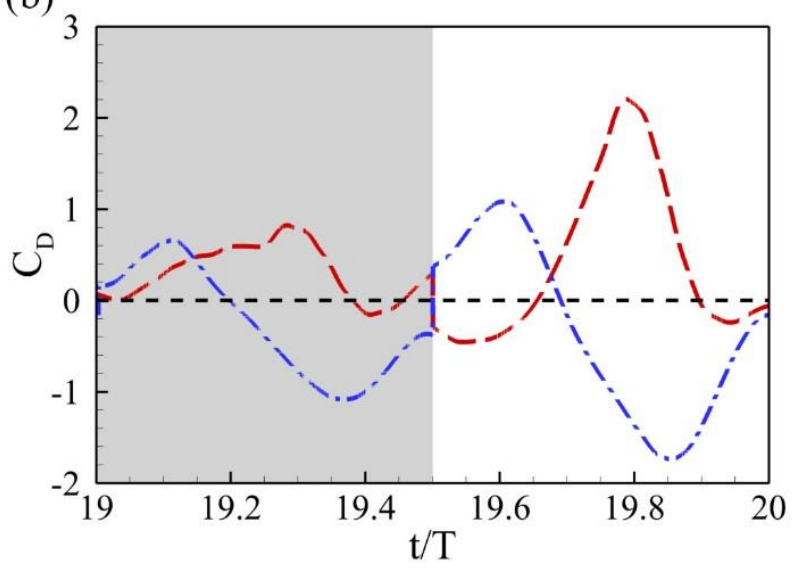

Figure 3-6. Time variation of relative force increments compared to the fully rigid plate. (a) $\beta_{T}=60^{\circ}, \varphi=60^{\circ}$. (b) $\beta_{T}=60^{\circ}, \varphi=-120^{\circ}$. 


\subsubsection{Effect of TEF Deflection Phase Difference}

To provide a comprehensive picture of how the TEF deflection phase difference affects overall unsteady phenomena, we compare the aerodynamic performance of two typical deformed plate configurations. The two selected cases correspond to positive (Figure 3-2 (c)) and negative (Figure 3-2 (d)) camber formations, respectively, with the same deflection amplitude $\left(\beta_{T}=60^{\circ}\right)$. As a reference, data for the corresponding fully rigid plate are also plotted in Figure 3-5. Their associated plate configurations are shown in Figure 3-2 (c-d) for an established cycle.

In general, the instantaneous force history (Figure 3-5 (a-b)) of all three cases shows two local maxima for each half-cycle. The first peak is associated with the wake capture at the beginning of the stroke, and the second peak is mainly caused by the delayed stall and rotational circulation mechanisms [38]. Between the two peaks is a local minimum referred to as a wake valley, which is caused by a combination of decreasing angle-of-attack and interaction with a pocket of downward momentum created during previous flapping cycles [83]. The asymmetrical force production between the down- and up-stroke is caused by irregularities in the vortex shedding and wing-vortex interaction [43].

Significant differences can be observed between the case with and without TEF and also between the positive and negative camber formations. By adding TEF deflection motion with different deflection phase difference value, the entire camber formation will be changed. This change will either delay or advance the dynamic stall. As a consequence, the location of the peak values of both forces and power coefficients will be shifted relative to that of the fully rigid case, as can be easily observed in Figure 3-5. 


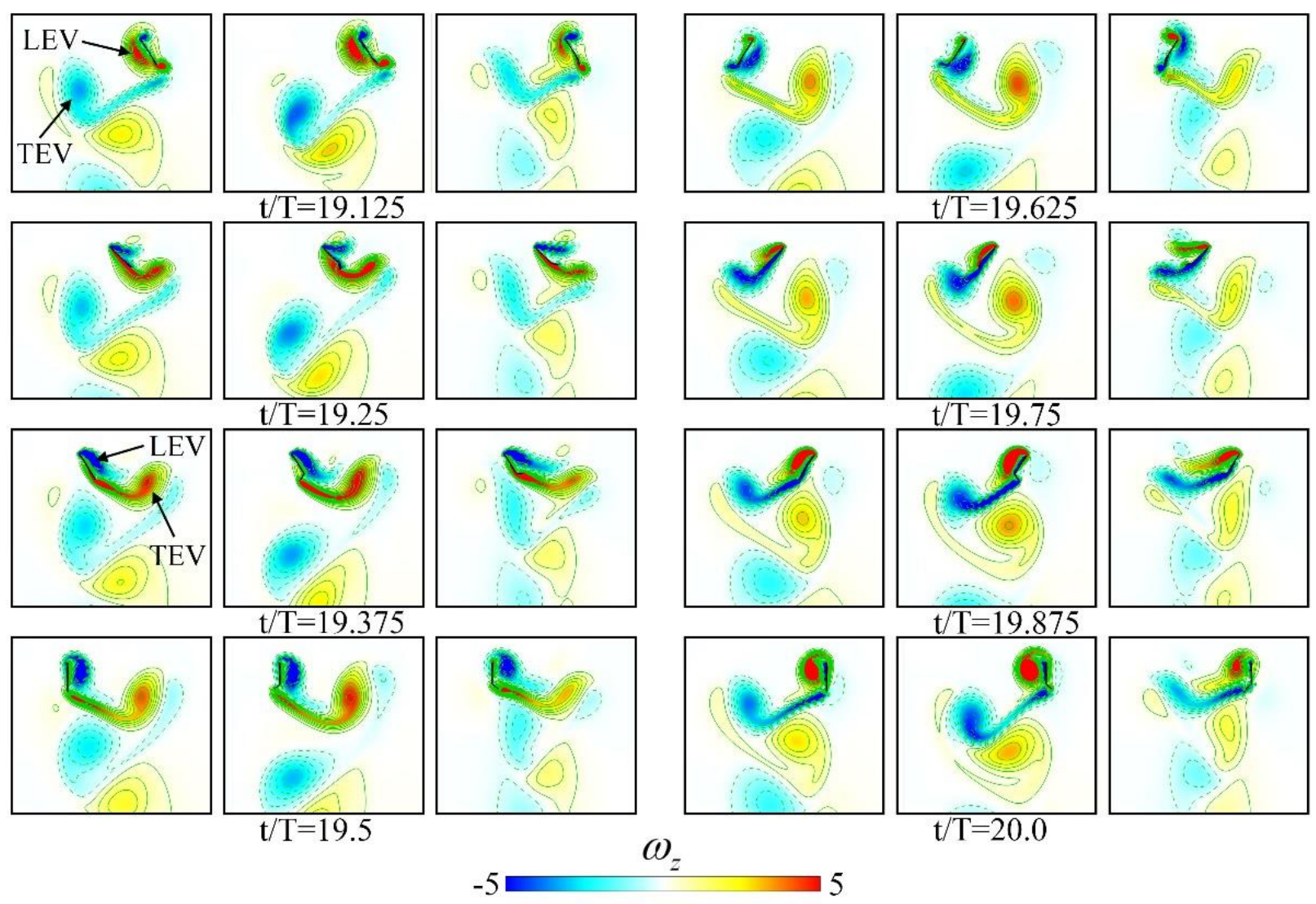

Figure 3-7. A series of instantaneous vortex field in a flapping cycle for a fully rigid plate (first and fourth columns), TEF deflection at $\beta_{T}=60^{\circ}, \varphi=60^{\circ}$ (second and fifth columns) and TEF deflection at $\beta_{T}=60^{\circ}, \varphi=60^{\circ}$ (third and sixth columns). The blue and red colors indicate clockwise and counterclockwise vortices, respectively.

Figure 3-5 (a) shows that, for the positive camber case, a significant lift enhancement occurs during the up-stroke relative to the fully rigid plate, whereas, the negative camber case shows a significant lift decrease. There are two possible reasons for this difference. First, the negative camber formation reduces the frontal area of the deformed plate, as shown in Figure 3-2 (d). Second, the reverse camber formation prevents the development of leading-edge vortex (LEV). 
For the latter, the comparison of vortical structure features would alleviate the problem and will be discussed in the following sections. Figure 3-5 (b) shows that greater drag is produced when the plate experiences higher lift. This result is common for unsteady mechanisms in hovering flight along a horizontal stroke plane. According to the previous literature, an increase in aerodynamic lift due to unsteady effects is typically accompanied by an increase in drag $[38,80]$. The power coefficient displays a similar trend as the drag coefficient varies, as shown in Figure 3-5 (c). This is because most of the energy has been consumed through drag producing rather than lift producing. For the negative camber case, the power consumption is much lower as a consequence of a lower frontal area.

To better understand the effect of the phase difference of TEF deflection on the force generation, Figure 3-6 shows the time course of relative force increments of the plates with two different TEF deflections compared to the fully rigid plate. In which, the relative force increments were obtained by subtracting the forces generated by the fully rigid plate. For the positive camber case $\left(\beta_{T}=60^{\circ}, \varphi=60^{\circ}\right)$, the maximum force increments happened in the middle of the downstroke and the upstroke. However, the negative camber case $\left(\beta_{T}=60^{\circ}, \varphi=-120^{\circ}\right)$ barely had force increments except during the one-eighth period after the plate reversal, but experienced significant force reduction at the mid-downstroke and the mid-upstroke. 
(a)

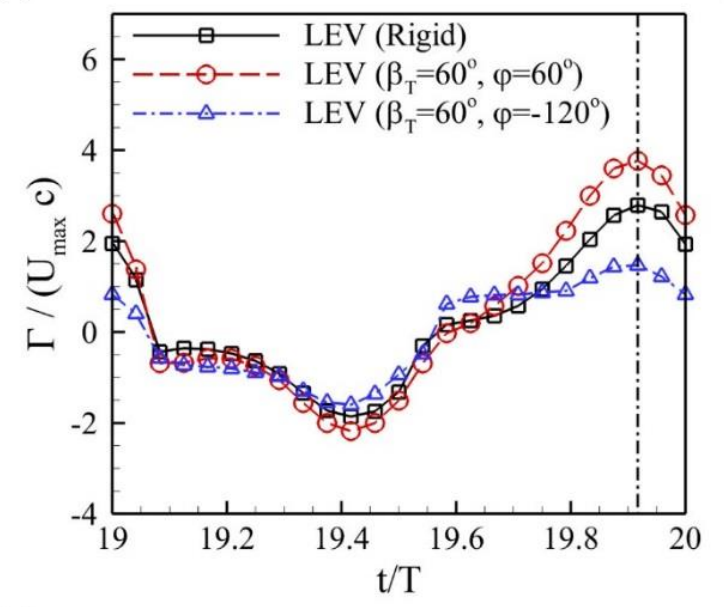

(c)

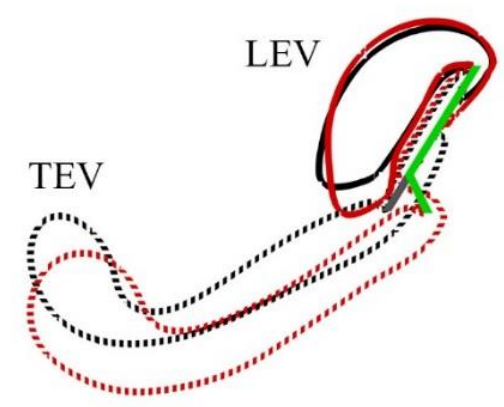

(b)

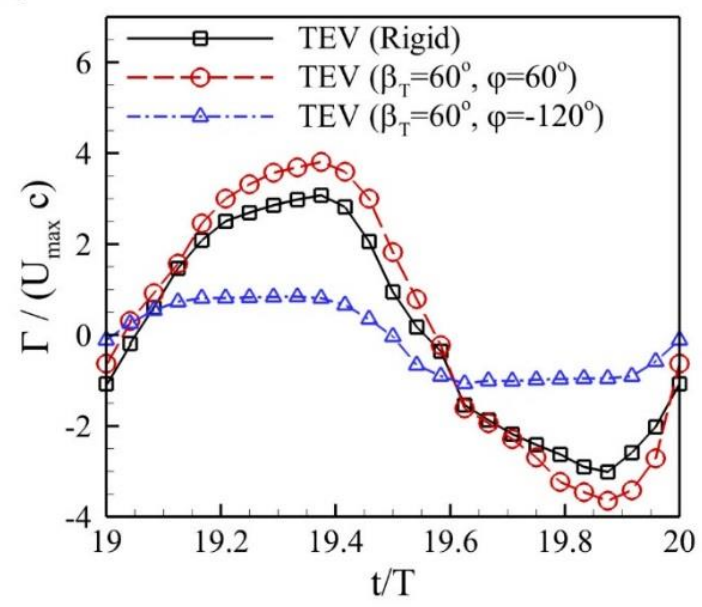

(d)

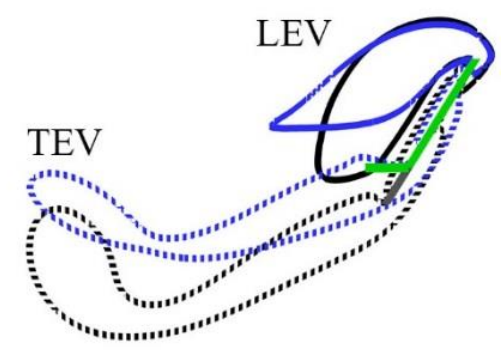

Figure 3-8. Comparison of LEV (a) and TEV (b) circulation. The vortex circulation is nondimensionalized by $U_{\max } c$. The positive and negative values correspond to the strength of the vortex during down-stroke and up-stroke, respectively. (c-d) Vortex contour at an instant labeled by the dashed-dot line in (a) for the fully rigid plate (black color), the TEF deflection at $\beta_{T}=60^{\circ}, \varphi=60^{\circ}$ (red color), and the TEF deflection at $\beta_{T}=60^{\circ}, \varphi=-120^{\circ}$ (blue color). The fully rigid and deformed plates are shown as gray and green color, respectively. The solid and dashed line indicates the LEV and TEV, respectively.

To understand the force enhancement mechanism caused by adding a dynamic TEF, we further investigate the near wake structures of all three cases as shown in Figure 3-7. The general 
trend of the vortex formation is similar among all cases. A leading edge vortex (LEV) is generated during each half-stroke and is then recaptured by the plate during its return trip after the stroke reversal. Then the LEV generated by the previous half flapping stroke moves downward along the plate and merges with the current trailing edge vortex (TEV). The merged vortex is strengthened and stretched by the trailing-edge. The wake below the hovering plate is marked by a pair of TEVs with opposite signs that are generated by the two half-strokes in a complete cycle.

The differences of these cases in the flow field are also evident. First, the positive camber case creates a larger size of LEV and TEV than others and its downwash presents a faster downward translational speed. In addition, the dynamic stall can be either advanced or delayed for different camber formations. During the translation phase (as shown in $\mathrm{t} / \mathrm{T}=19.375$ and $\mathrm{t} / \mathrm{T}=19.875$ ), the LEV of the negative camber case presents a clear detachment from the plate suction side. On the contrary, the positive camber case shows a better LEV attachment comparing to the fully rigid plate. Furthermore, during the stroke reversal, the positive camber case create a relative stronger wake region over the other cases, which implies the enhancement of the wake capture mechanism (i.e., the negative vortex blob at $\mathrm{t} / \mathrm{T}=19.5$ and the positive blob at $\mathrm{t} / \mathrm{T}=20.0)$.

To quantify the strength of the LEV, we further visualize the vorticity field using contour lines. After each vortex is manually identified, a closed contour line is generated around this vortex with a specified level, and the circulation $(\Gamma)$ is then calculated by integrating along this line. Although the magnitude of the circulation depends on the chosen contour level, the characteristic behavior of the vortex is not affected by this choice. The negative and positive signs represent the vortex rotation direction, which can be either clockwise or counterclockwise. 
(a)

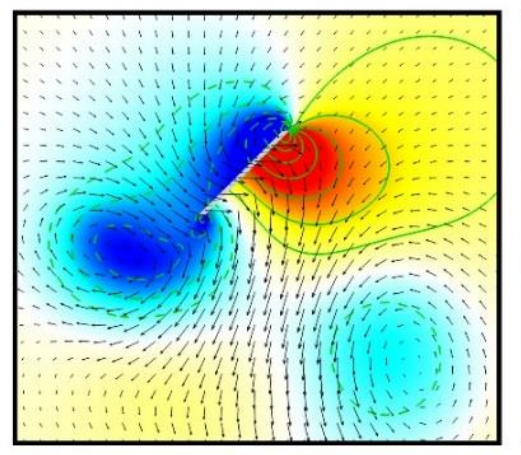

(b)

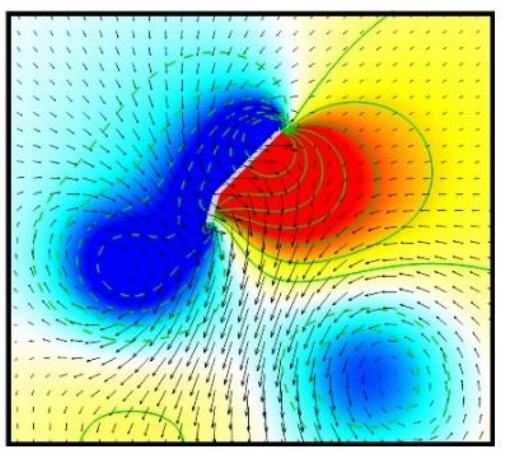

(c)

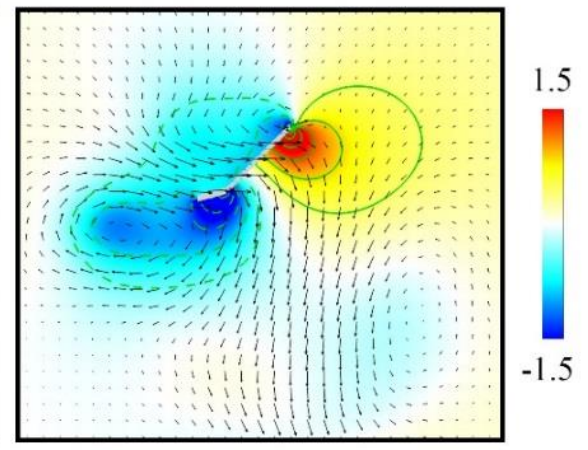

Figure 3-9. Comparison of pressure distribution and velocity vector at $t / T=19.75$. (a) Fully rigid plate, (b) Positive cambered plate at $\beta_{T}=60^{\circ}, \varphi=60^{\circ}$, and (c) Negative cambered plate at $\beta_{T}=60^{\circ}, \varphi=-120^{\circ}$. The pressure is normalized by $\rho U_{\max }^{2}$.

Figure 3-8 (a-b) shows a comparison of LEV and TEV circulation corresponding to the vortices shown in Figure 3-7. In general, the strength and timing of the vortices have significant effects on force production. The peak timing of the TEV circulation appears slightly earlier than that of the LEV circulation. It can be observed that the LEV and TEV for the positive camber case are much stronger than the corresponding vortices for the fully rigid case over a significantly long period. In terms of the flow physics, the wake capture mechanism is enhanced partially due to a stronger flow around the plate at stroke reversal. Note that both enhancement periods appear during the second half of each stroke, when the camber deformation is formed. Unlike that in the positive camber case, the vortex circulation in the negative camber case is much weaker, and the magnitude of LEV circulation is comparable between the down- and upstrokes. 
Figure 3-8 (c-d) compares the contour lines of LEV and TEV for an instant that is labeled as a dashed-dot line in Figure 3-8 (a). Because the magnitude of the circulation is proportional to the area of the contour lines, the difference in circulation can be visualized by comparing the areas of the contour lines. It is observed that the TEF dynamic motion directly stretches its local vortex structure and feeds extra circulation to the TEV (dashed blue line), which results in forming a relatively larger LEV pair. This local flow change also pulls down the LEV structure closer to the plate compared with that observed in the fully rigid case. It is worth noting that the enhancement of LEV and TEV through positive cambering observed in the current work is in line with previous studies on wing flexibilities. These include numerical simulations of insectlike cambering effects of a 2-D wing [84], experimental measurements of robotic rotating wings with varying flexural stiffness [85], and flow-structure interaction of vein-stiffened flapping wings [41]. On the other hand, for the negative camber case, the TEF stretches its TEV upward. As a consequence, the LEV is pulled up and detached from the suction side of the plate.

The abovementioned local flow modulation will directly alter the pressure distribution. From the normalized pressure contours (Figure 3-9), we can see that the positive cambered plate creates a larger low-pressure area on the suction side and the pressure difference between the upper and lower plate surface is clearly increased. The velocity vectors indicate that the positive cambered plate generates a stronger downwash in the flow field comparing to the other two cases. The distribution of the pressure and velocity vector entail the lift generation. Hence, the positive cambered case at this instant experiences a relative larger vertical force, as shown in Figure $3-5$ at $\mathrm{t} / \mathrm{T}=19.75$. On the opposite, the negative camber case generates a stronger backflow on the suction side and this will pull up the LEV. As a result, the detachment of LEV (dynamic stall) makes the overall force production of the negative camber case drops significantly. 


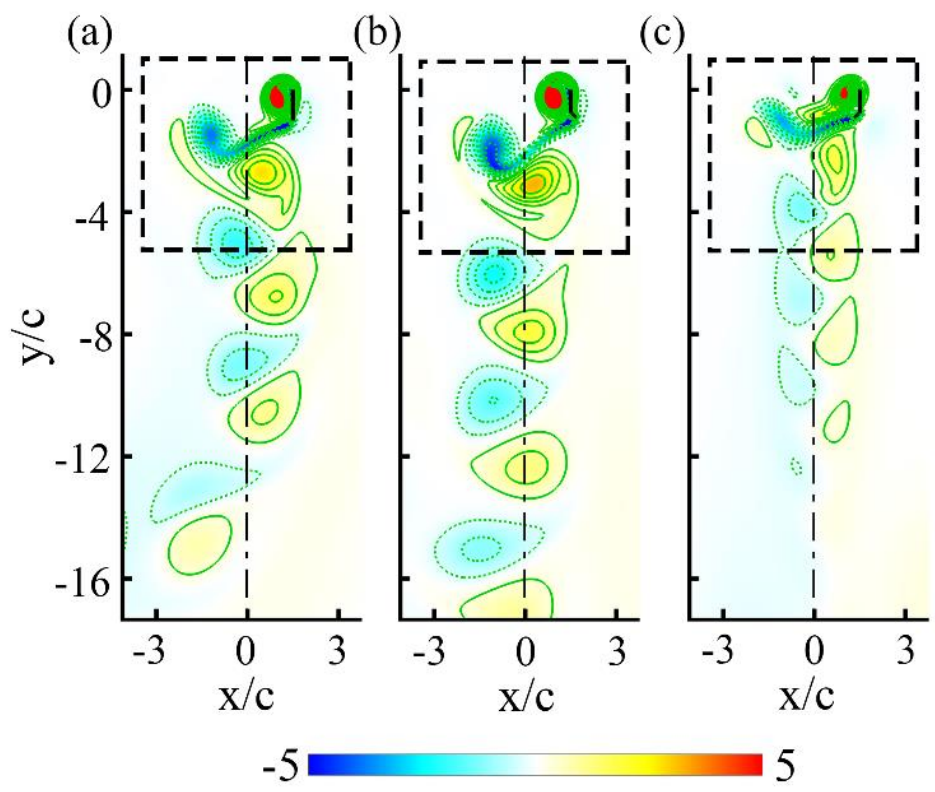

Figure 3-10. Far wake vortical structure comparison of fully rigid plate (a), TEF deflection at $\beta_{T}=60^{\circ}, \varphi=60^{\circ}$ (b) and TEF deflection at $\beta_{T}=60^{\circ}, \varphi=-120^{\circ}$ (c) at $\mathrm{t} / \mathrm{T}=20.0$. The dashed square corresponds to the region shown in Figure 3-7.

To demonstrate the global impact of the dynamic TEF deflection, Figure 3-10 presents the far wake vortical structure. After long-term development, the far-field flow presents a reverse Karman vortex street for all three cases. The positive camber formation results in a more vigorous vortex street (Figure 3-10 (b)) throughout the entire evolution period. The corresponding overall vortex shedding trajectory presents a slight deflection. It can also be observed that the shedding vortex pair translates faster in the vertical direction. On the other hand, the negative camber case shows an enervated vortex pattern (Figure 3-10 (c)) with slower translational speed. The shedding vortex gradually vanishes because of the viscosity. In its downwash devolution, the vortex street stays on the same side of the symmetry line of the wake. 


\subsubsection{Effect of TEF Deflection Amplitude}

Unlike deflection phase difference effect, adjusting the deflection amplitude will result in a change of camber magnitude. In this section, the effect of deflection amplitude on aerodynamic performance is investigated at $\beta_{T}=15^{\circ}, 30^{\circ}, 60^{\circ}, 90^{\circ}$ for positive camber (at $\varphi=60^{\circ}$ ) and negative camber (at $\varphi=-120^{\circ}$ ) configurations, respectively. The instantaneous forces and power coefficients are plotted in Figure 3-11. The performance of a fully rigid plate is also re-plotted as a reference. The corresponding vorticity fields are shown in Figure 3-12.

In Figure 3-11, the first peak of each stroke resulting from the wake capture presents a clear enhancement for the higher deflection amplitude $\left(\beta_{T}=60^{\circ}, 90^{\circ}\right)$ but not for the lower deflection amplitude. The second peak of each stroke, which is caused by the delayed stall and rotational circulation mechanisms, shows a gradually increasing trend with deflection amplitude, but it drops when the TEF is over-deflected at $\beta_{T}=90^{\circ}$. For negative camber formation, the first peak increases along with the deflection amplitude, but the second peak presents a decreasing trend at the same time. Although the deflection amplitude change will alter the peak value and appearance timing at certain levels, the overall trend of the instantaneous force and power coefficients for both the positive and negative camber formations are essentially the same. Figure 3-12 presents the vortical structures for both the positive and negative camber formations at various deflection amplitudes. The plots show that although the change in deflection amplitude will slightly deflect the vortex street, it will not alter the overall flow pattern dominated by the deflection phase difference. 
(a)

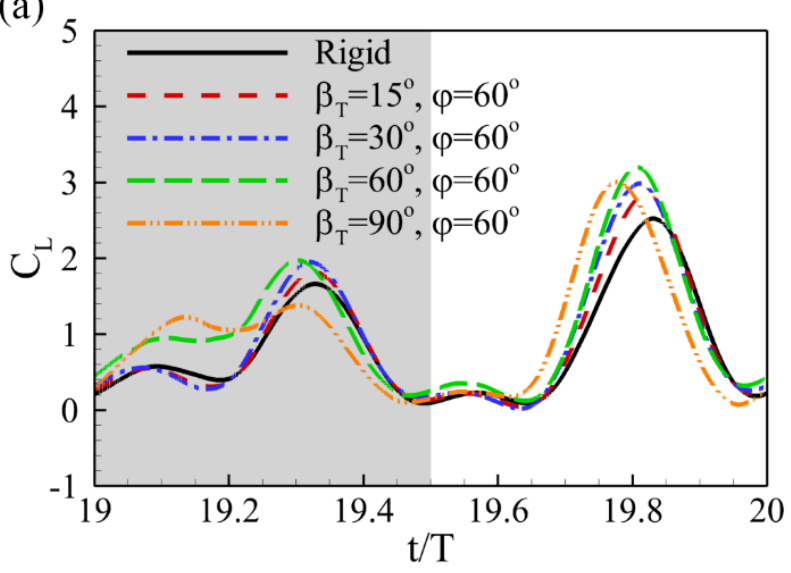

(c)

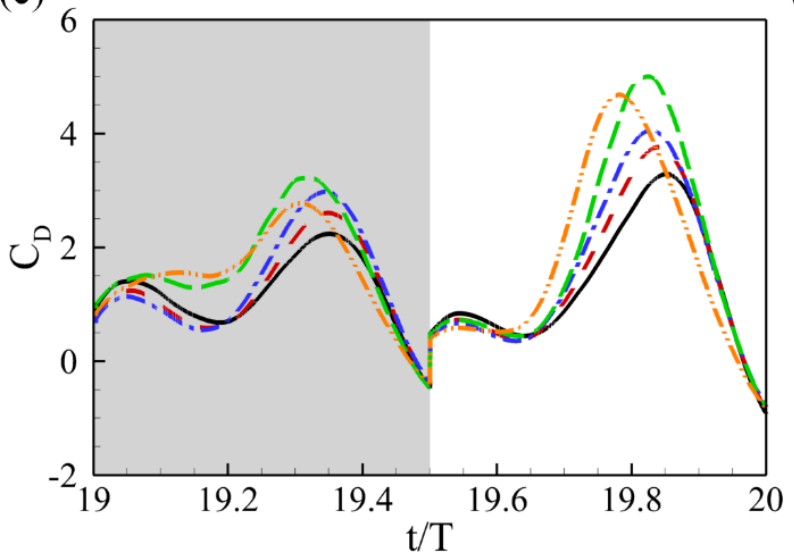

(e)

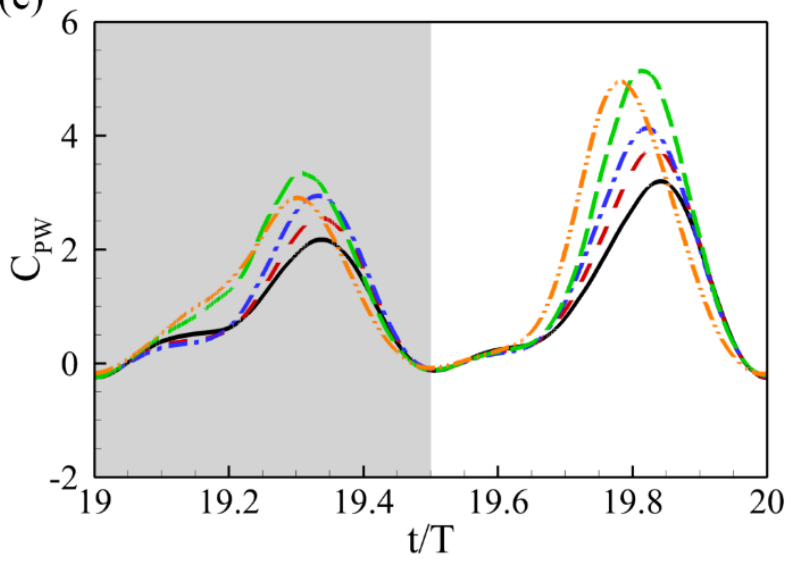

(b)

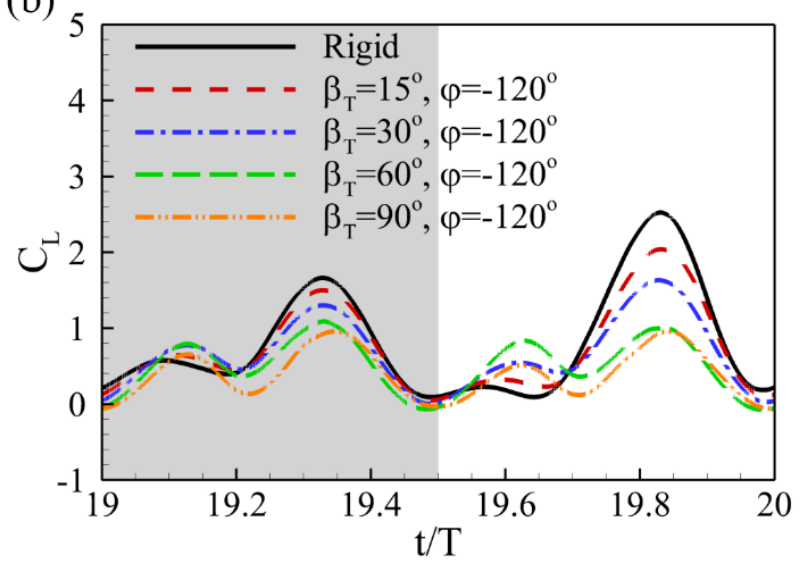

(d)

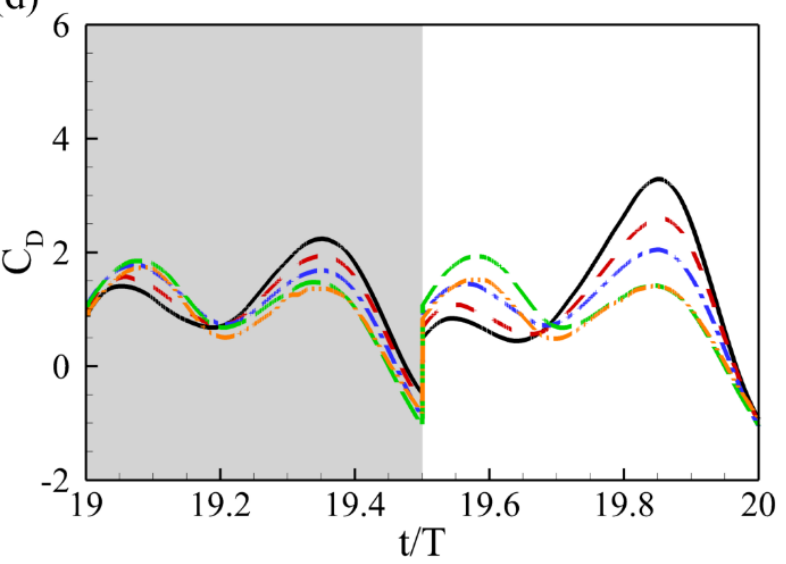

(f)

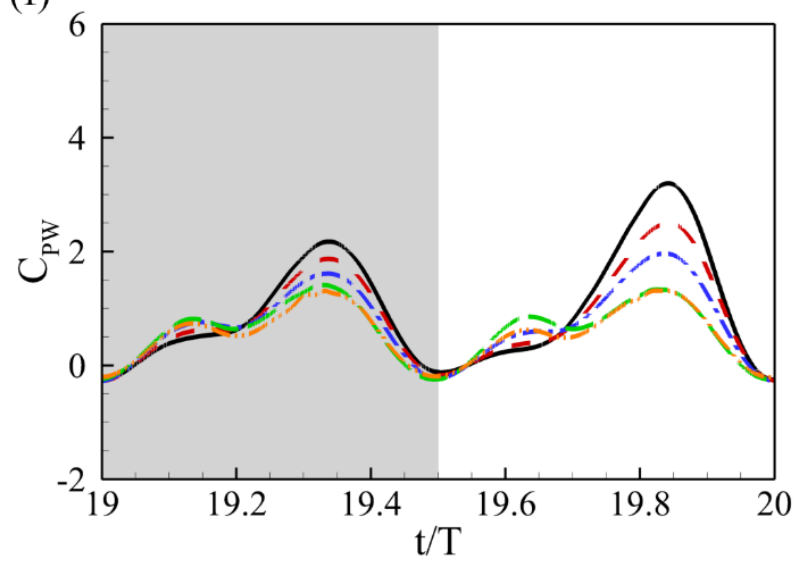

Figure 3-11. Time traces of lift (a-b), drag (c-d), and power (e-f) coefficients for positive and negative camber formations at different deflection amplitudes. 


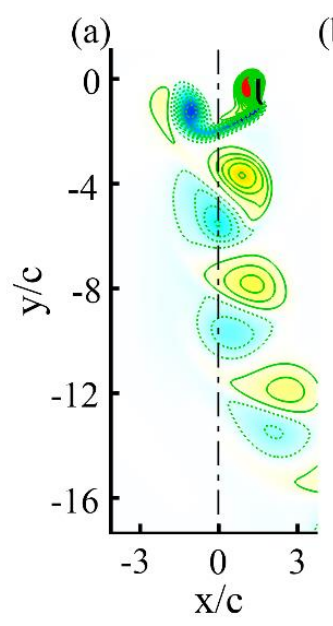

(b)

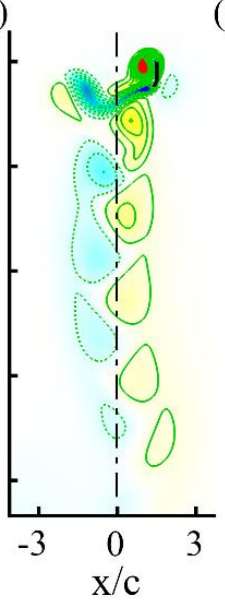

(c)

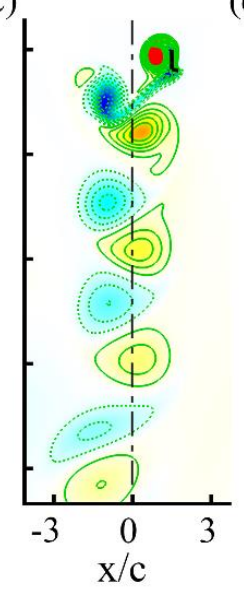

(d)

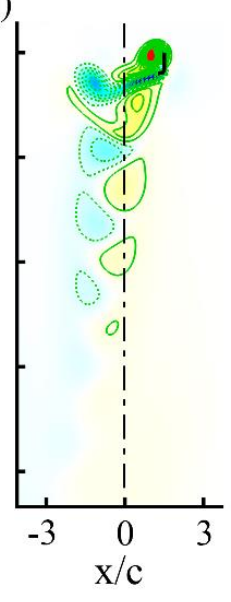

5

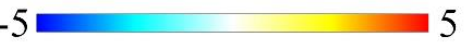

(1)

Figure 3-12. Far wake vortical structure comparison of TEF deflection at $\beta_{T}=30^{\circ}, \varphi=60^{\circ}$ (a), $\beta_{T}=30^{\circ}, \varphi=-120^{\circ}$ (b), $\beta_{T}=90^{\circ}, \varphi=60^{\circ}$ (c), and $\beta_{T}=90^{\circ}, \varphi=-120^{\circ}$ (d).

To systematically explore the effects of TEF on aerodynamic performance, Figure 3-13 (ab) shows the lift and drag coefficients averaged over 20 flapping cycles. The results are shown for the TEF deflection amplitudes $\beta_{T}=15^{\circ}, 30^{\circ}, 60^{\circ}, 90^{\circ}$ and deflection phase difference $\varphi$ values ranging from $-180^{\circ}$ to $180^{\circ}$. The performance of a fully rigid plate is indicated by the dash-dot line. It can be observed that for all deflection amplitudes, the TEF deflection phase difference has a significant effect on the force production. Specifically, the optimal lift appears over the range of $\varphi=\left(0^{\circ}, 90^{\circ}\right)$, and the peak value is up to $26 \%$ higher than that of a fully rigid plate. The deflection phase difference range $\varphi=\left(0^{\circ}, 90^{\circ}\right)$ also leads to an increase of at least $40 \%$ in the drag coefficient compared with that of the fully rigid plate. Although drag production is not desirable for the steady motion, it has the potential to enhance maneuverability if an asymmetric deflection phase difference value is selected between the inner and outer flapping- 
wings because the torque generation for inset flight is mainly drag-based [86]. For variations in the deflection phase difference over the ranges $\left(-180^{\circ},-90^{\circ}\right)$ and $\left(90^{\circ}, 180^{\circ}\right)$, both the lift and drag decrease, especially for the larger deflection amplitude cases. This decrease is observed due to the decrease in the frontal area when a reverse camber is formed for $\varphi$ over these ranges.

(a)

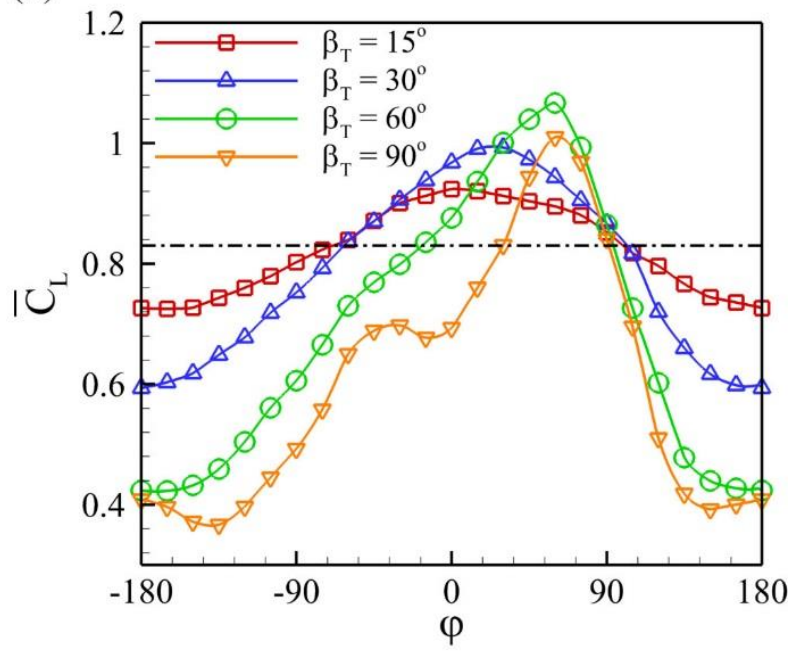

(c)

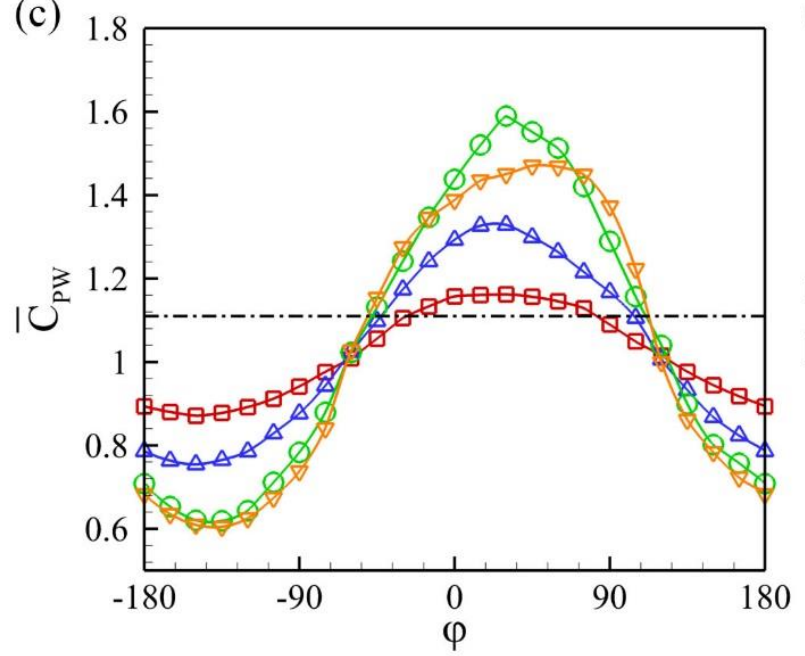

(b)

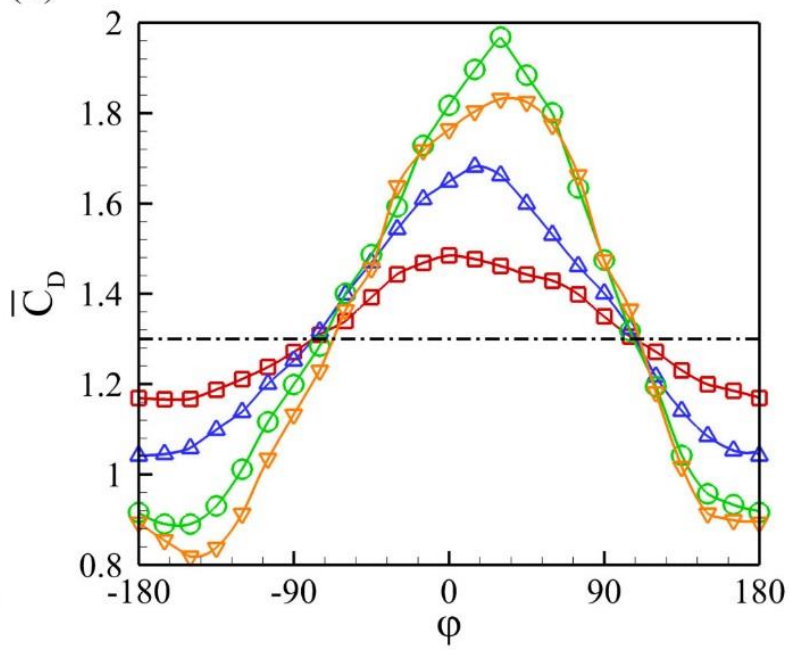

(d)

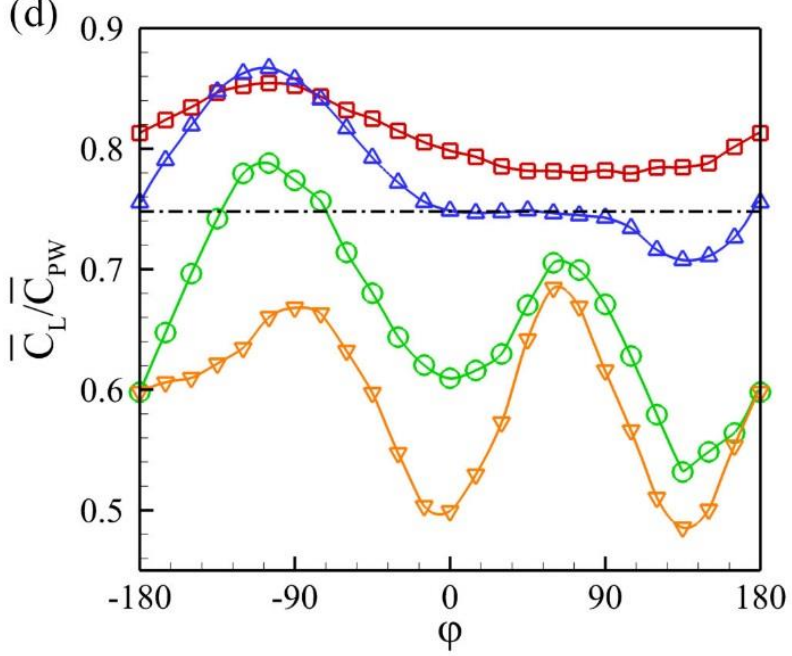

Figure 3-13. Aerodynamic performance of plate at $H_{L}=0.75 c$. Cycle-averaged lift (a), drag (b), and power (c) coefficients, and lift-to-power ratio (d). 
In Figure 3-13 (c), the power calculation is based on the input from the leading edge of the plate, and negative power is not taken into account. Overall, the power coefficient displays a trend similar to that of the drag coefficient. This is again because most of the energy is consumed through drag rather than through lift. Unlike the force and power coefficient maps, in which higher values always appear at higher deflection amplitudes, the lift-to-power ratio shown in Figure 3-13 (d) presents a higher value at lower deflection amplitudes, especially for a negative camber formation. The optimal lift-to-power ratio can reach up to 0.85 , which is $21 \%$ higher than that for the fully rigid plate. The possible reason is that although the negative camber cases own lower lift production, the symmetric flow pattern (i.e., Figure 3-10 (c)) reduces the power consumption in the horizontal direction. This leads to a higher aerodynamic efficiency in terms of the lift-to-power ratio.

Comparing the four deflection amplitudes, we find that there is an optimal value for the magnitude of camber formation. For lift production, a moderate deflection amplitude $\left(\beta_{T}=60^{\circ}\right.$ ) with positive camber formation generates the optimal lift along with the decrease of the lift-topower ratio. However, the low deflection amplitude $\left(\beta_{T}=15^{\circ}\right)$ with a positive camber is able to improve both the lift and lift-to-power ratio, but at much smaller level than that of $\beta_{T}=60^{\circ}$. It should be noted that the camber formation at $\beta_{T}=15^{\circ}$ corresponds to a maximum $8 \%$ camber-tochord ratio, where the camber height is defined as the distance between the hinge locations to the straight line connecting leading to trailing edge. This value is close to that observed in real insect wings [87].

Moreover, as shown in Figure 3-13, the phase difference of the TEF deflection is another important parameter for controlling the plate's aerodynamic performance. The change of this 
parameter during flapping is equivalent to the time variation of the camber formation, as shown in Figure 3-2 (b). Results have shown that the optimal lift production always occurs when the deflection phase difference is within $0^{\circ}<\varphi<90^{\circ}$ (Figure 3-13 (a)). Meanwhile, the maximum camber is formed when the wing is about to reversal. This is consistent with previous findings in real insect flights. Table 3-1 shows the comparison of camber formation including camber-tochord ratio $\left(h_{\max } / c\right)$ and formation timing $\left(\tau_{h \max }\right)$ of the maximum camber between the optimal lift case from the current work and previous studies on real insects. Here, $h_{\max }$ and $c$ represent the maximum camber height and wing chord length, respectively, and $\tau_{h \max }$ is the formation timing of the maximum camber which is normalized by the downstroke duration $\left(T_{\text {down }}\right)$. Results have shown that for various real insects, the maximum camber-to-chord ratios are between 5\% $16 \%$, and they are all formed at the end of the downstroke, $0.7 T_{\text {down }} \sim 0.9 T_{\text {down }}$. Similar trends can also be found from the results of the optimal lift case in the current work despite that the TEF motion is prescribed.

It is worth noting that the insect wings are made of complicated biomaterials coupled with ingenious wing structural designs that are able to extract maximum advantage from elastic tailoring and other mechanisms during the flapping motion. Current work has indicated that by carefully choosing the control parameters, active control of wing surface morphing may be able to achieve biological levels of aerodynamic performance as insect flapping wings. This observation can be treated as a promising inspiration for the wing design of flapping-winged MAVs. 
Table 3-1: The maximum camber-to-chord ratio $\left(h_{\max } / c\right)$ and the timing of maximum camber $\left(\tau_{h \max }\right)$ during downstroke for real insects along with the optimal lift case from the current study.

\begin{tabular}{|c|c|c|c|}
\hline & Flight conditions & $h_{\max } / c$ & $\tau_{h \max }$ \\
\hline $\begin{array}{c}\text { Dragonfly hindwing } \\
\text { [87] }\end{array}$ & Free flight & $5 \%-9 \%$ & 0.73 \\
\hline $\begin{array}{l}\text { Desert locust forewing } \\
\qquad[88]\end{array}$ & Tethered flight & $2 \%-9 \%$ & 0.68 \\
\hline $\begin{array}{l}\text { Desert locust hindwing } \\
\qquad[88]\end{array}$ & Tethered flight & $5 \%-10 \%$ & 0.72 \\
\hline $\begin{array}{c}\text { Hoverfly } \\
\text { [89] }\end{array}$ & Free flight & $6 \%-11 \%$ & 0.71 \\
\hline $\begin{array}{c}\text { Butterfly } \\
\text { [90] }\end{array}$ & Forward flight & $7 \%-15 \%$ & 0.78 \\
\hline $\begin{array}{c}\text { Beetle hindwing } \\
\text { [91] }\end{array}$ & Free flight & $11 \%-16 \%$ & 0.90 \\
\hline $\begin{array}{c}\text { Honeybee } \\
\text { [92] }\end{array}$ & Free flight & $5 \%-12 \%$ & --- \\
\hline $\begin{array}{c}\text { Current work } \\
\left(\beta_{T}=60^{\circ}, \varphi=60^{\circ}\right)\end{array}$ & Hovering & $15 \%$ & 0.82 \\
\hline
\end{tabular}

\subsubsection{Effect of Hinge Location}

In previous sections, the discussion is focused on a flapping plate with a hinge located at three-quarter chord length $\left(H_{L}=0.75\right)$ from the leading edge. In this section, the effects of varying hinge location on aerodynamic performance are investigated. For making a fair 
comparison, we keep the same effective pitching angle for different hinge location cases. The effective pitching angle (as shown in Figure 3-14) is defined in the same way as that used in [40]. For different hinge location cases, the total length of the whole plate is still maintained as unit one.

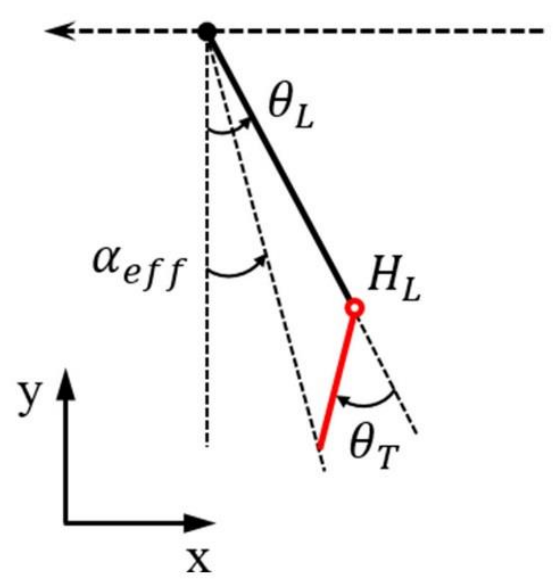

Figure 3-14. Definition of effective pitching angle $\left(\alpha_{\text {eff }}\right)$.

Figure 3-15 shows the mean aerodynamic performance for different hinge locations. By keeping the same effective pitching angle, the deflection amplitude for varying hinge location is different. For instances, the deflection amplitude $\left(\beta_{T}\right)$ of hinge location at 0.25 , and 0.5 chord length from the leading edge is around $18.5^{\circ}$ and $27.8^{\circ}$, respectively, for achieving the same effective pitching angle as hinge placed at three-quarter chord length cases at $\beta_{T}=60^{\circ}$. In general, the aerodynamic performance follows the same trend for varying hinge locations. However, there still exists slight difference especially for the lift generation. It is demonstrated that changing hinge locations have a different impact on the aerodynamics of positive and negative camber formations. 
(a)

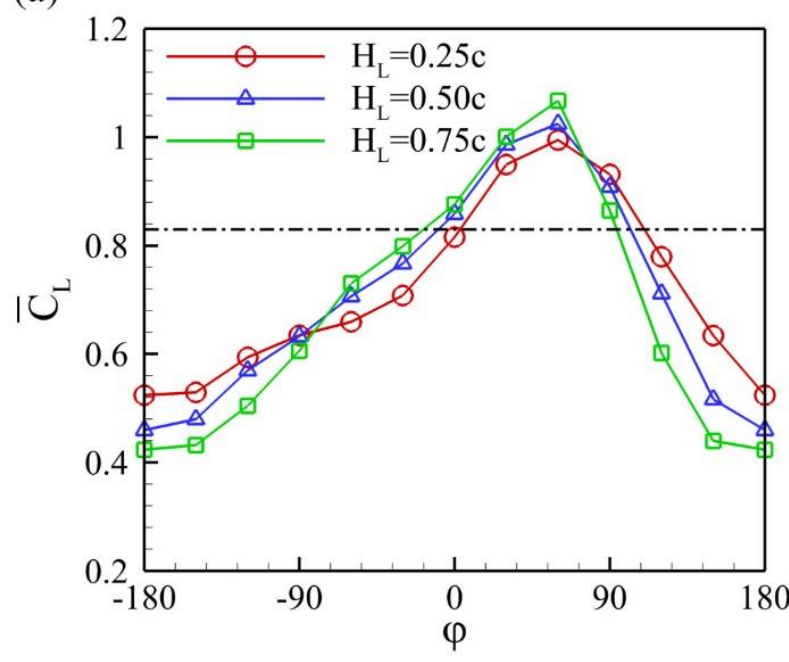

(c)

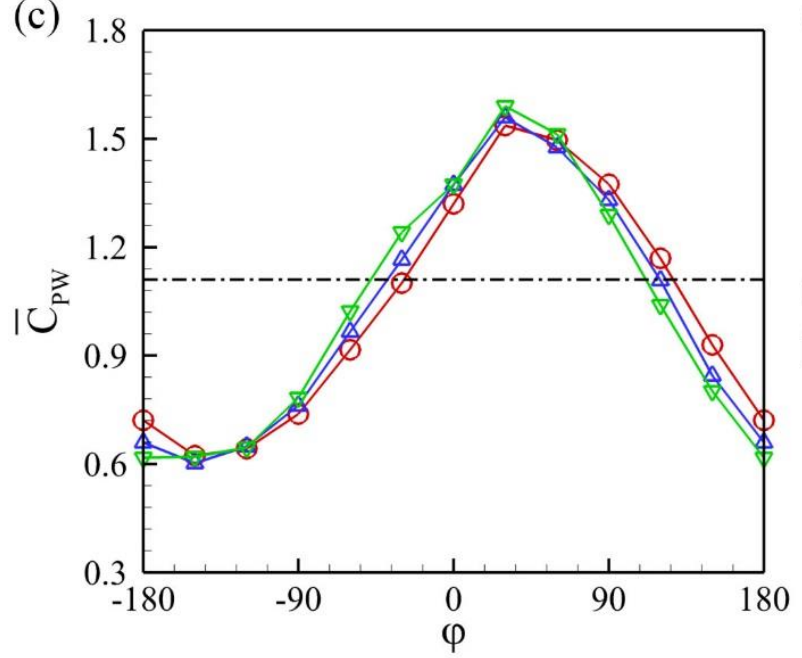

(b)

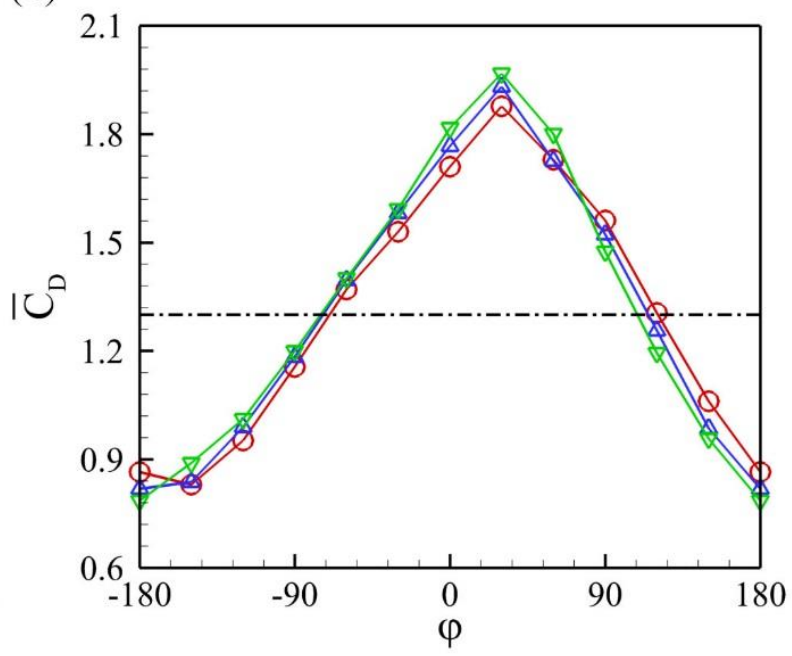

(d)

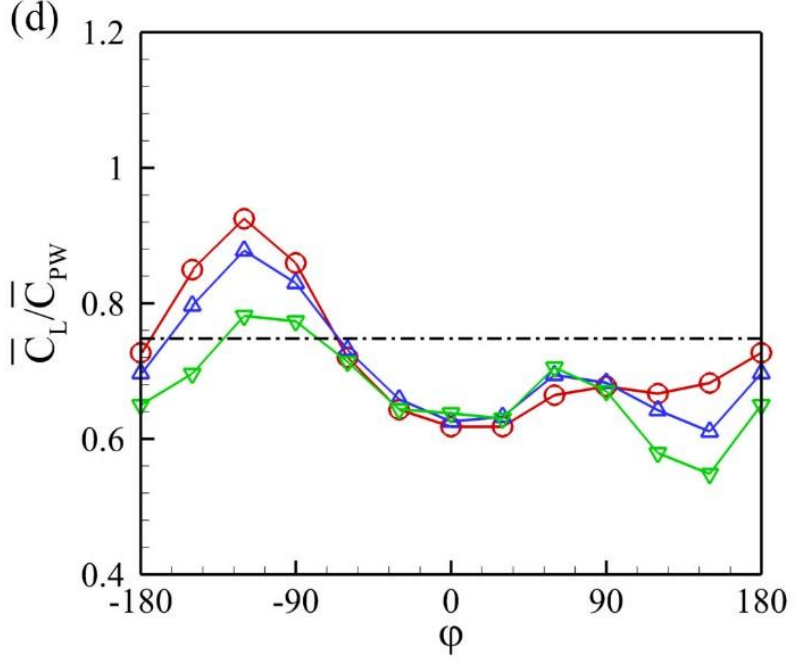

Figure 3-15. Aerodynamic performance of plate has different hinge locations. Cycle-averaged lift (a), drag (b), and power (c) coefficients, and lift-to-power ratio (d).

Figure 3-15 (a) shows that for positive camber, $H_{L}=0.75$ yields the highest lift, whereas for negative camber cases, $H_{L}=0.25$ leads to the greatest lift. More specifically, the averaged lift enhancement of positive camber cases at $H_{L}=0.75$ is $7.2 \%$ higher than the same case at 
$H_{L}=0.25$. In contrast, the averaged lift for the negative camber formation at $H_{L}=0.25$ is $9 \%$

higher than the same case at $H_{L}=0.75$. In addition, the drag and power coefficients in Figure

3-15 (b-c) only show slight differences between various hinge locations because the effective angle of attack for all simulations is kept the same. Moreover, the difference in the lift-to-power ratio (as shown in Figure 3-15 (d)) is directly determined by the change in lift production.

\subsubsection{Effect of Reynolds Number}

In order to study the Reynolds number ( $R e$ ) effects, we have changed $R e$ from 50 to Numerical Investigation of Energy Extraction in a Tandem Flapping Wing Configurationand run the higher Reynolds number cases on finer meshes. At each Reynolds number, simulations are performed at $H_{L}=0.75 \mathrm{c}$ and $\beta_{T}=60^{\circ}$ for different deflection phase difference.

Figure 3-16 (a-c) show comparisons of the cycle-averaged forces and power coefficients at various $R e$. In general, the lift coefficient increases with Reynolds number, especially for positive camber formation. However, the drag and power coefficients present different trends for positive and negative camber cases respectively when Reynolds number increases. For positive camber cases, both the drag and power coefficients increase with Reynolds number, whereas both the drag and power coefficients decrease as $R e$ increases for negative camber formation. In Figure 3-16 (d), the lift-to-power ratio always shows an increasing trend with the Reynolds number for different deflection phase difference values. This trend reveals that with the increase in Reynolds number, the dynamic TEF gains more benefit from the unsteadiness of the sounding flow. Although there is a difference in magnitude for the cases with different Reynolds numbers, the overall performance trend for varying deflection phase difference remains the same. 
(a)

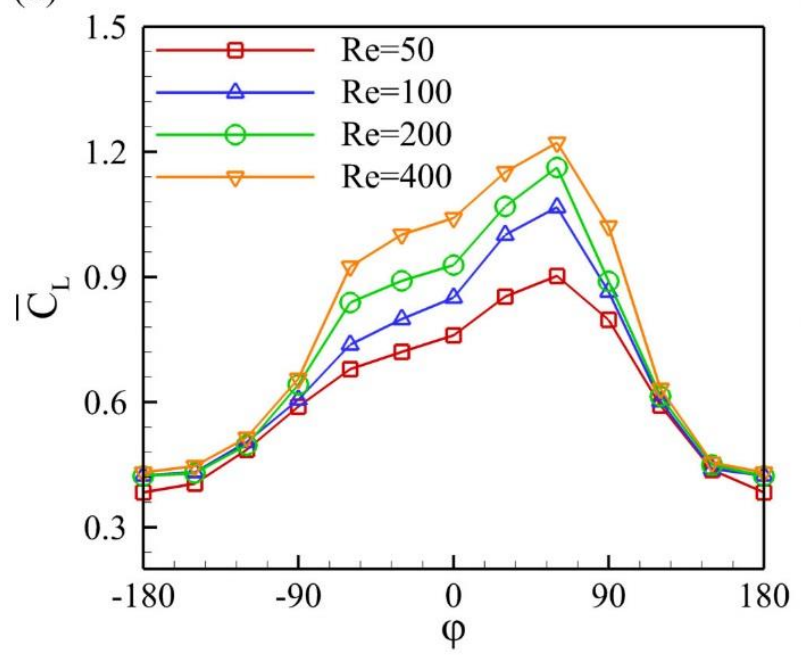

(c)

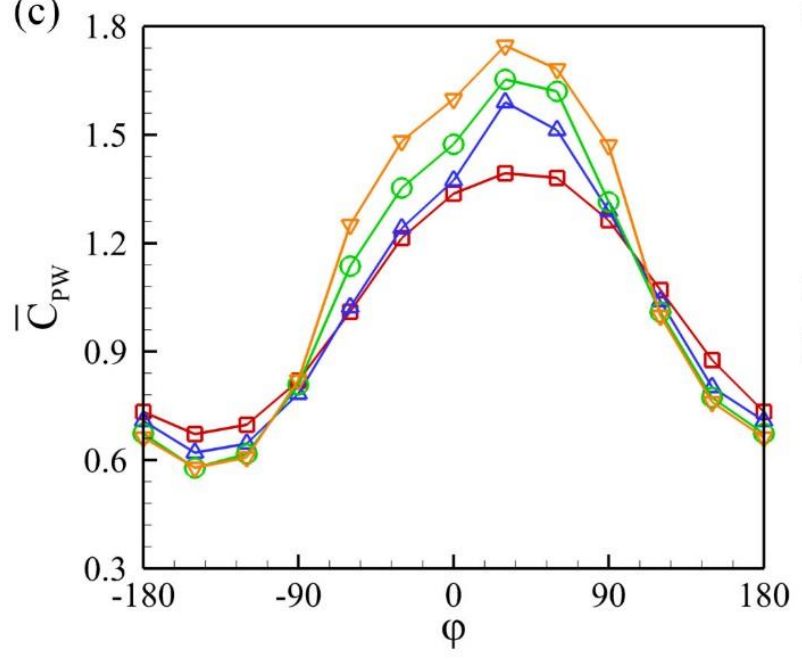

(b)

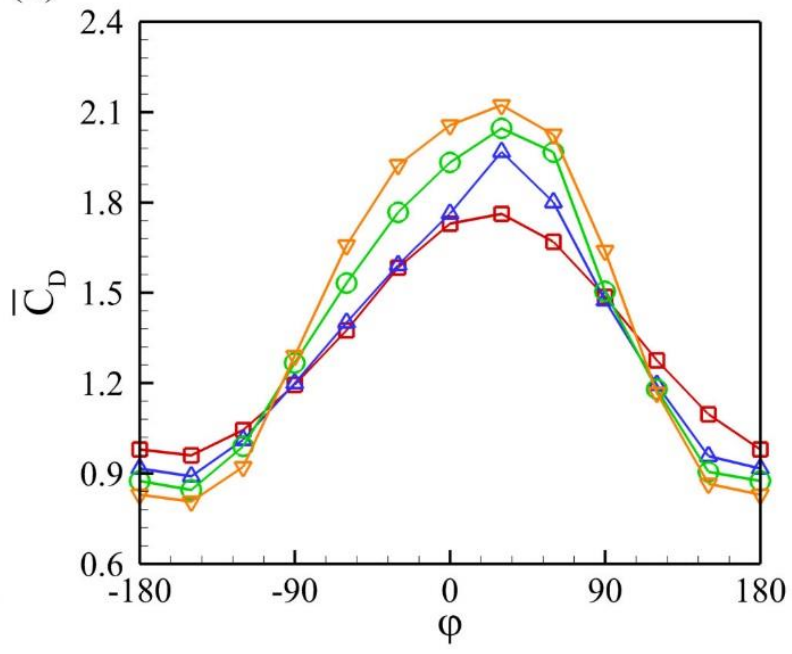

(d)

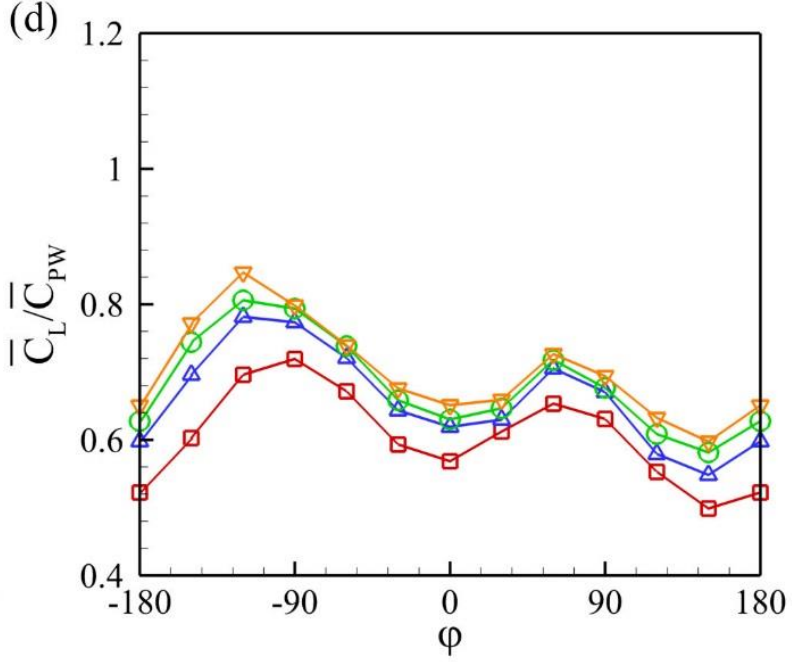

Figure 3-16. Aerodynamic performance of plates with various deflection phase difference at $\beta_{T}=60^{\circ}$ for different Reynolds numbers. Cycle-averaged lift (a), drag (b), and power (c) coefficients, and lift-to-power ratio (d). 
(a)

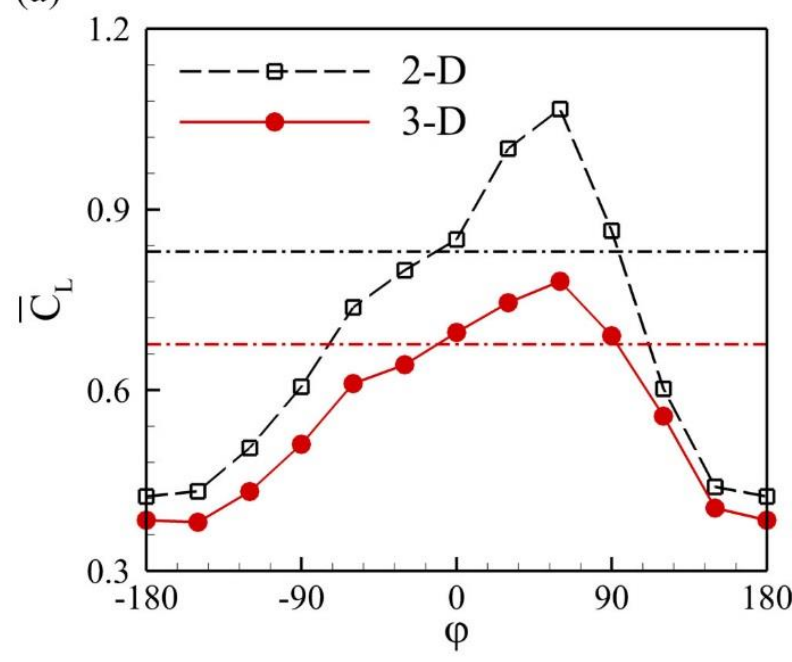

(c)

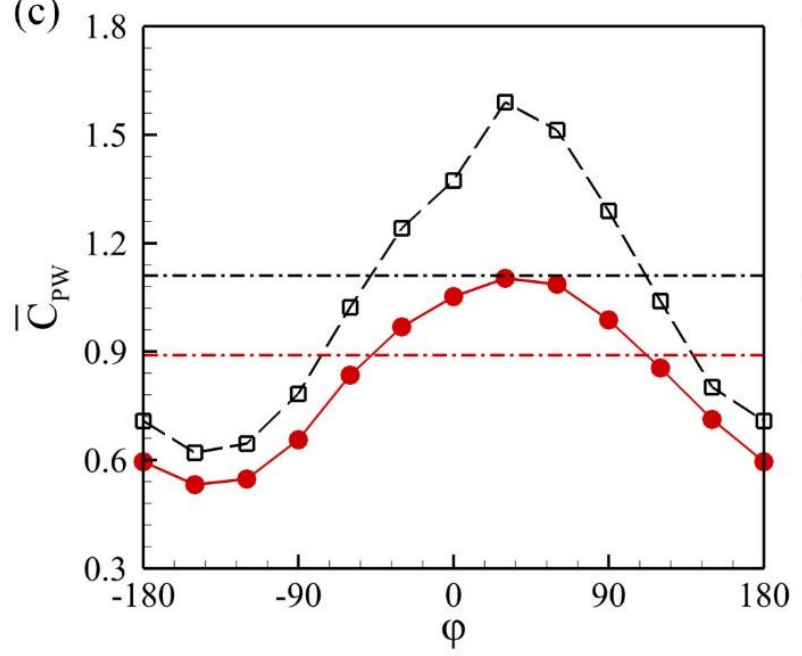

(b)

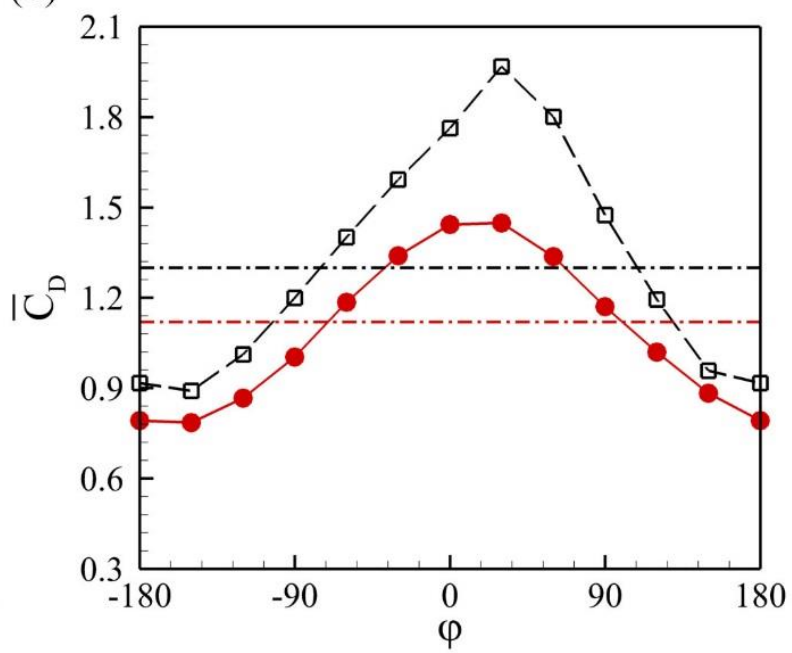

(d)

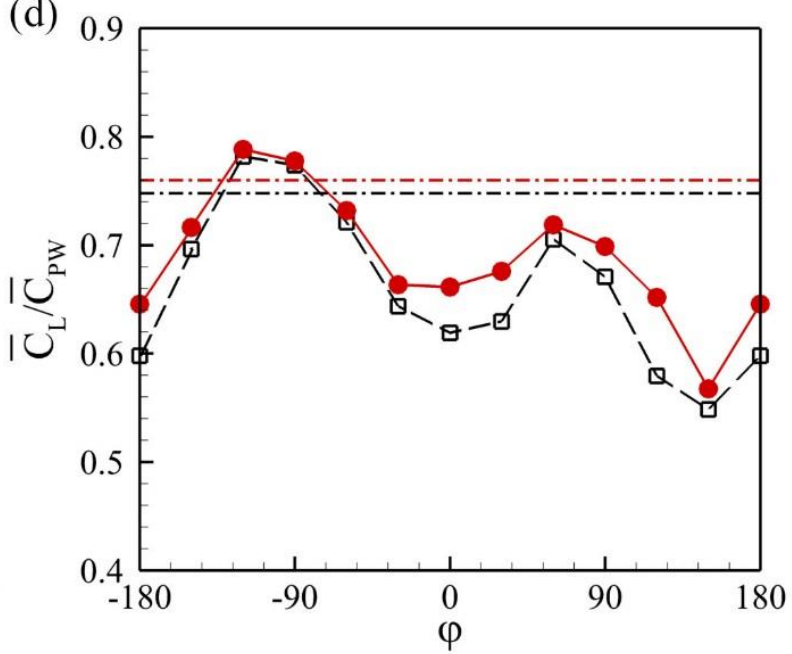

Figure 3-17. Comparison of aerodynamic performance between 2-D plate and 3-D plate $(A R=2)$ at $\beta_{T}=60^{\circ}$ for different TEF deflection phase difference. Cycle-averaged lift (a), drag (b), and power (c) coefficients, and lift-to-power ratio (d).

\subsubsection{Effect of Three-dimensionality}

At last, the effects of TEF three-dimensionality on the aerodynamic performance and flow modulation are investigated. Computations are performed using an $A R=2$ plate at $R e=100$ with 
$H_{L}=0.75 c, \beta_{T}=60^{\circ}$ and various $\varphi$ for total four flapping cycles. A notable difference between the 3-D simulation and 2-D simulation is that the 3-D flow quickly reaches a nearly periodic state after approximately two cycles. In the following discussion, the mean forces and power coefficients for 3-D cases are calculated based on the averages of the last two cycles.

(a)

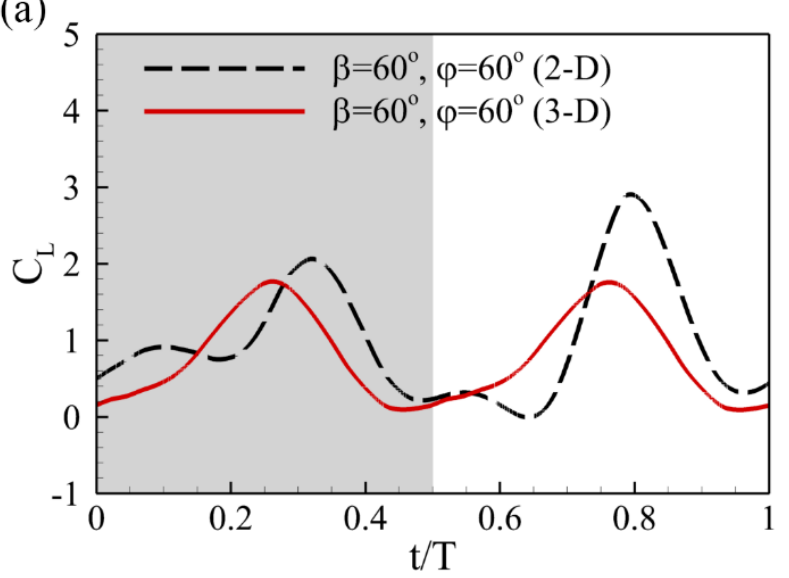

(c)

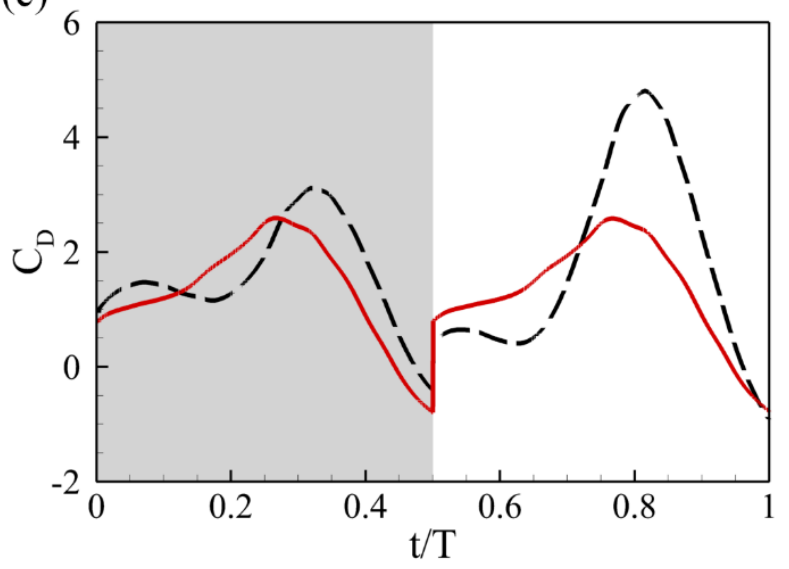

(b)

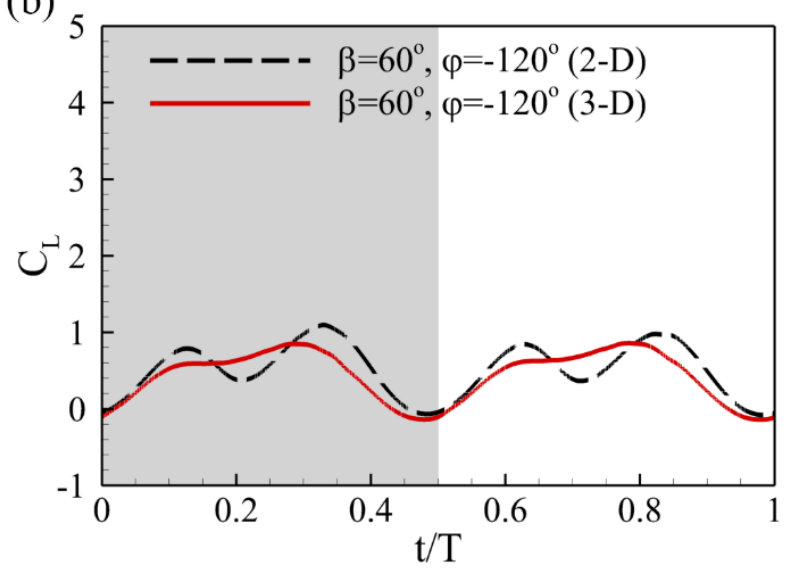

(d)

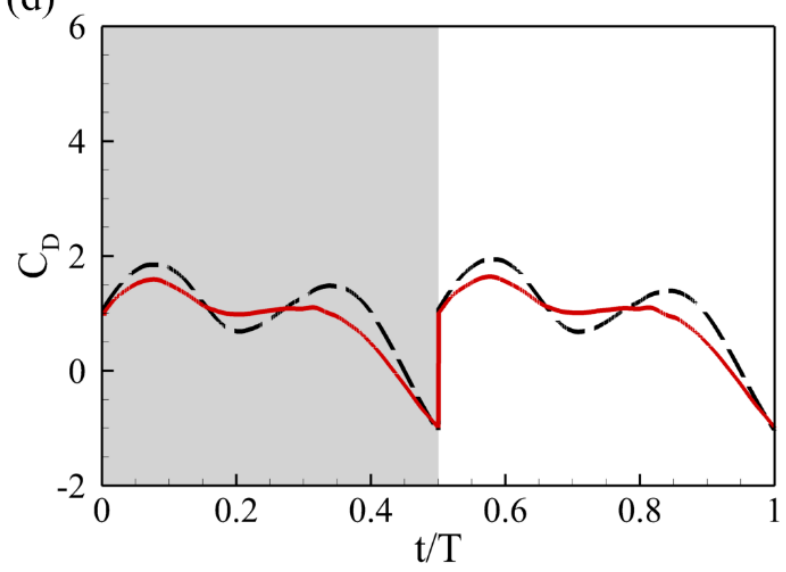

Figure 3-18. Comparison of instantaneous lift (a-b) and drag (c-d) coefficients between the 2-D and 3-D cases for positive and negative camber formations.

Figure 3-17 shows the mean force, power coefficients and lift-to-power ratio for 3-D cases at various deflection phase difference. The corresponding 2-D cases are re-plotted for 
comparison. In general, the overall trend demonstrated by the 3-D cases follows that of the 2-D cases but with smaller amplitude. Based on previous studies, the force fluctuation per unit span is suppressed in 3-D cases due to the existence of tip vortices [93], which results in a smaller mean force and smaller power coefficients.

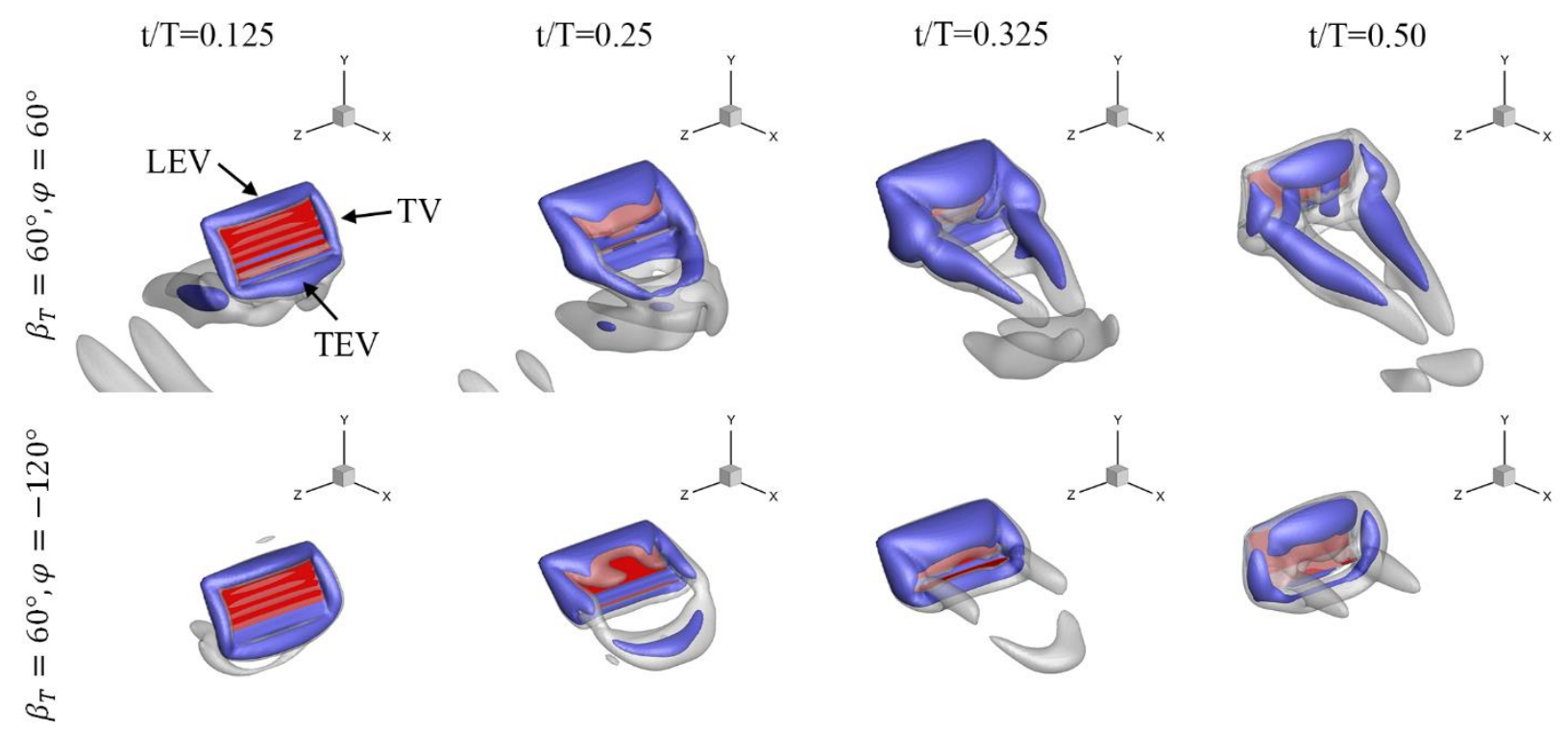

Figure 3-19. Comparison of vortical structures between positive $\left(\beta_{T}=60^{\circ}, \varphi=60^{\circ}\right)$ and negative $\left(\beta_{T}=60^{\circ}, \varphi=-120^{\circ}\right.$ ) camber formations at selected instants during the down-stroke of the $4^{\text {th }}$ flapping cycle.

The time histories of force coefficients at $\beta_{T}=60^{\circ}$ and $\varphi=60^{\circ},-120^{\circ}$ are plotted in Figure 3-18 to help us better illustrate the difference between 3-D and 2-D cases. First, the double peaks of each half-stroke for the 2-D cases merge into a single peak in the 3-D cases. In addition, the force production for each down- and up- stroke is comparable in 3-D cases. A possible reason is that due to the absence of the span-wise flow and tip vortices, the 2-D case is much more 
sensitive to the surrounding flow field and vortex interactions than its 3-D case. Moreover, in 3D, the tip vortex and span-wise flow stabilizes the LEV and prevents it from shedding [4].

Figure 3-19 shows 3-D wake structures obtained using the iso-surface of Q-criteria [94] at selected instants for both positive and negative camber cases. Two iso-surfaces are plotted to highlight the inner core $(\mathrm{Q}=3.0)$ and outer shell $(\mathrm{Q}=1.0)$ of the vortex structure. The corresponding instantaneous force histories are presented in Figure 3-18. Because both the force history and vortex formation are symmetric during the down- and up-strokes, only the downstroke vortex formation is presented here. In general, the flow structures is similar as reported in previous literature [95]. At the beginning of the down-stroke, the flow separates from the leading edge and tips, which act as strong sources of vorticity. Vorticity is gradually fed into the surrounding flow field, and forms the leading-edge, trailing-edge and tip vortices as vortex sheets rolling up from each edge. As the leading-edge vortex (LEV) grows, the trailing-edge vortex (TEV) and symmetric tip vortices (TVs) together form a vortex ring at the bottom of the plate. With the growth of the vortex structure, the plate gradually reaches the peak lift. During the second half of the down-stroke, the LEV vortex starts to detach from the suction side of the plate due to the continuous increase of the plate pitching angle. As a consequence, the force starts to drop and gradually reaches its minimum value. The comparison of the vortex structure between positive and negative cases reveals that the positive camber case $\left(\beta_{T}=60^{\circ}, \varphi=60^{\circ}\right)$ forms a larger LEV with relatively stronger TVs than the negative camber case $\left(\beta_{T}=60^{\circ}, \varphi=-120^{\circ}\right)$. The same phenomenon can be observed from the slice cuts of the vorticity field.

In Figure 3-20, the slice cuts are taken at the tip, a distance of $0.5 \mathrm{c}$ from the tip, and at mid- 
span during the down-stroke. As in the 2-D cases, the LEV of the positive camber case shows better LEV attachment than that in the negative camber case, as indicated by the snapshots captured at $\mathrm{t} / \mathrm{T}=0.25$. During the translation process, the positive camber formation enhances the tip vortex and creates a stronger LEV. Furthermore, a double core vortex $($ at $\mathrm{t} / \mathrm{T}=0.325)$ is formed at the tip when a positive camber is formed, which might be because of the enhancement of the tip vortex. It is also observed that the shed vortices are more dissipative in the 3-D case than in the 2-D case by comparing the vorticity plots in the far wake (Figure 3-10).

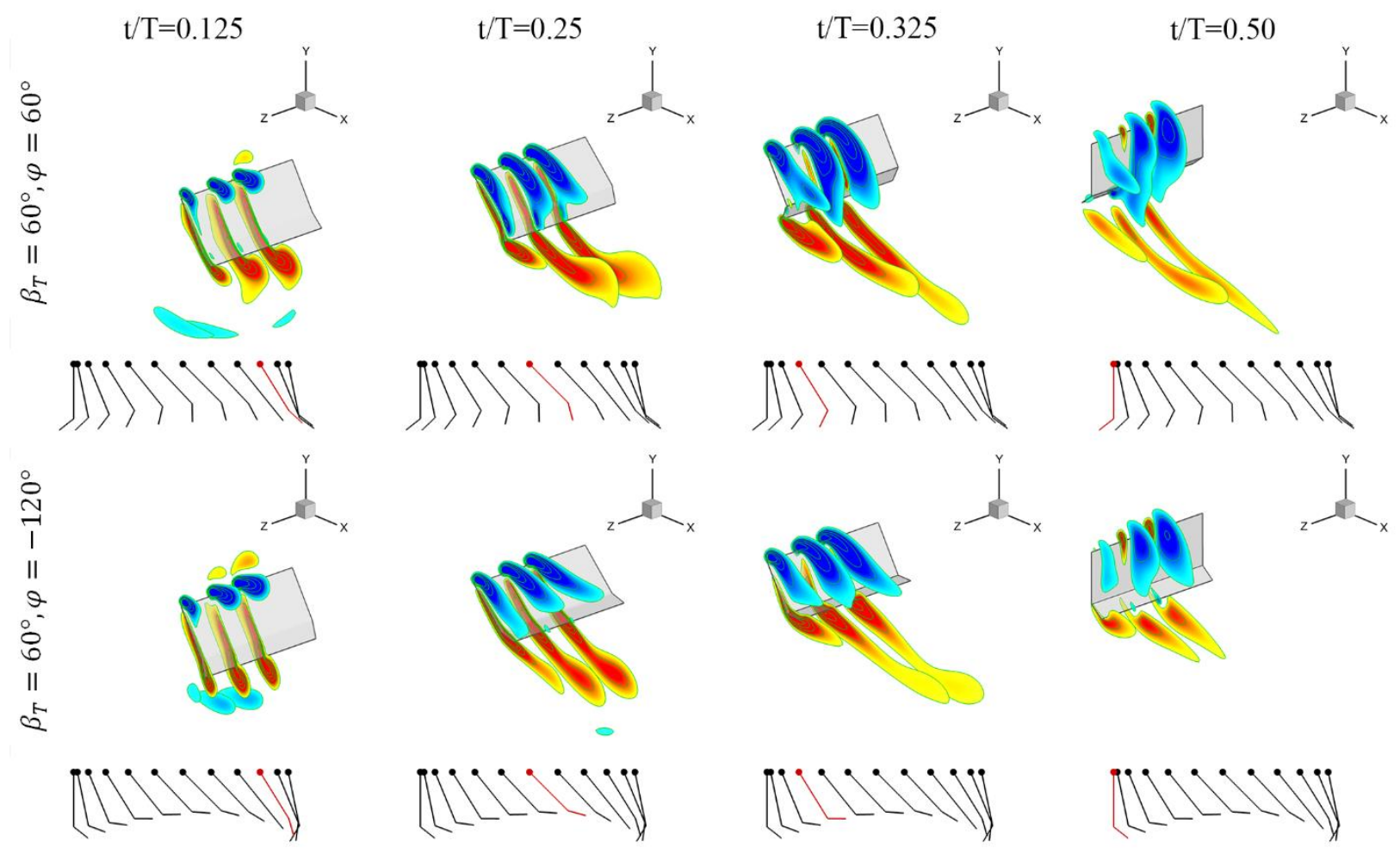

Figure 3-20. Comparison of Z-vorticity contours between positive $\left(\beta_{T}=60^{\circ}, \varphi=60^{\circ}\right)$ and negative $\left(\beta_{T}=60^{\circ}, \varphi=-120^{\circ}\right)$ camber formation at selected time instants during the downstroke of the $4^{\text {th }}$ flapping cycle. The slices cuts are taken at plate tip, at a distance of $0.5 c$ from the tip, and at the mid-span. 


\subsection{Chapter Summaries}

The effects of the dynamic trailing-edge flap (TEF) on the aerodynamic performance and flow structures of both 2-D and 3-D flapping plates has been numerically studied for Reynolds number range of 50 to 400 . Various parameters for controlling TEF kinematics, such as deflection phase difference, deflection amplitude, and hinge location are considered. Our results suggest that the TEF deflection phase difference play a more dominant role than other parameters. The lift enhancement always happens for positive camber formation cases (i.e., deflection phase difference $\left.\varphi \in\left(-90^{\circ}, 90^{\circ}\right)\right)$. By tailoring the deflection phase difference and deflection amplitude, the lift production can be increased up to $26 \%$ comparing to a fully rigid plate. The analysis of the associated unsteady flow structures has shown that the dynamic motion of TEF is able to feed extra circulation into the trailing-edge vortex which induces a stronger counterpart leading-edge vortex. As a result of local flow modulation by TEF, the vortex on the suction side is pulled down closer to the plate (stall delay), which leads to the improvement of lift production. Our results also suggest that the plate performance is insensitive to the hinge location when the effective pitching angle kept the same. Changing Reynolds numbers only affects the magnitude of mean force coefficients whereas the overall trend of TEF effect maintains the same. Results from the low-aspect-ratio plates indicate that the general conclusion drawn from 2-D cases is applicable to 3-D cases even though the flow features are different at certain levels. 


\section{Wake Topology and Propulsive Performance of Bio-inspired}

\section{Pitching-Rolling Plates}

Flapping motion is widely utilized by many kinds of biological propulsors, including fish pectoral fins [96,97,98] and insect/bird wings [61,99]. Such motion generally consists of an oscillatory rolling motion about a fixed joint with a simultaneous change in the geometric pitch angle with respect to the spanwise axis. As pointed out in previous studies [26,33], pitchingrolling plates could serve as an ideal canonical model for investigating the aero-/hydro-dynamics of bio-inspired flapping propulsion. To achieve a better understanding of pitching-rolling propulsors, we extend our previous studies on pitching-heaving plates [26,27] to investigate the wake topology and propulsive performance of low-aspect-ratio plates over a range of Strouhal numbers, Reynolds numbers, aspect ratios, and phase difference angles between pitching and rolling motion. To advance our knowledge of vortex dynamics, the proper orthogonal decomposition analysis is also used to investigate the wake topology of pitching-rolling plates.

\subsection{Problem Definition}

The current study employs elliptic plates with a finite aspect ratio $(A R)$ and a plate thickness of 3\% of the chord length (a similar plate geometry was also used in Ref.[27]). The elliptic plate is defined by two major diameters: the chord length $c$ and the span length $S$. The $A R$ of the plate is defined as the square of the span $(S)$ divided by the planform area of the plate $\left(A_{\text {plan }}\right)$ and equals $4 S / \pi c$. The surface of the plate is represented by a fine unstructured mesh with triangular elements. 
(a)
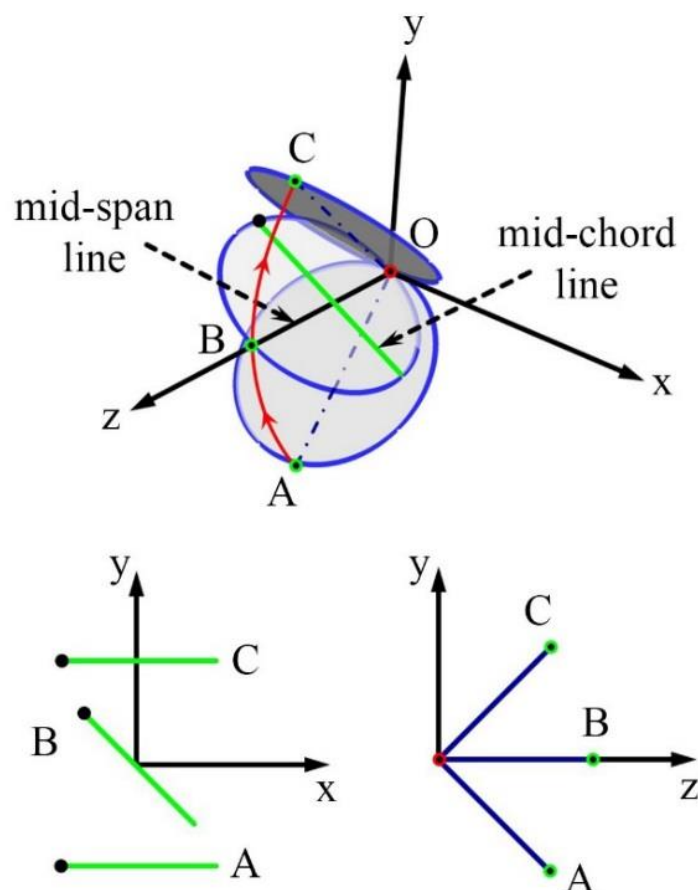

(b)
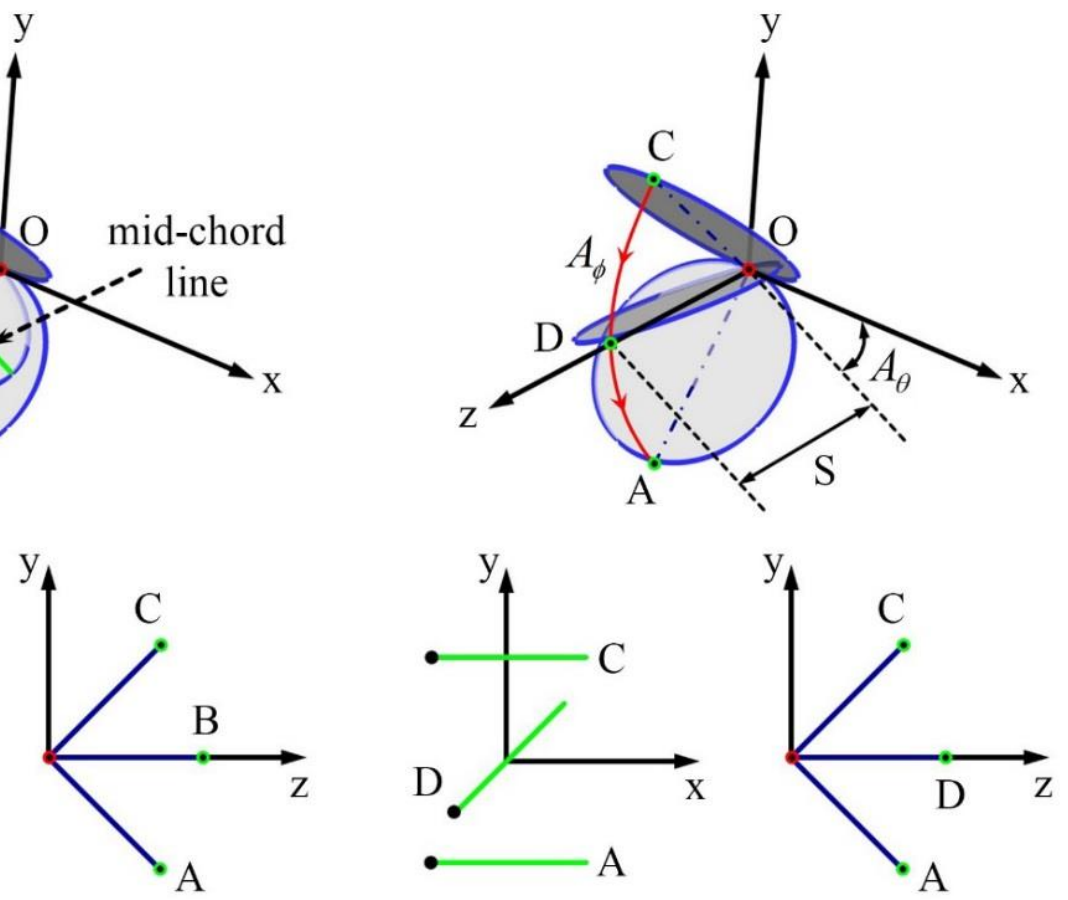

Figure 4-1. Schematic of the plate kinematics during the upstroke (a) and the downstroke (b), respectively.

The 3-D plate undergoes combined rolling and pitching motions in the yz-plane, as shown in Figure 4-1. In Figure 4-1 (a), the plate is rolling upward from the lowest position (A) to the highest position (C) with instantaneous pitching angle change. The maximum pitching angle is reached at the mid-stroke position (B). To better illustrate the pitching-rolling motion, the schematic of corresponding mid-chord and mid-span line kinematics are also provided at the bottom of Figure 4-1 (a). Similar as the upstroke motion, Figure 4-1 (b) presents the downward rolling motion of the plate and the minimum pitching angle is reached at another mid-stroke position (D) during the downstroke. 


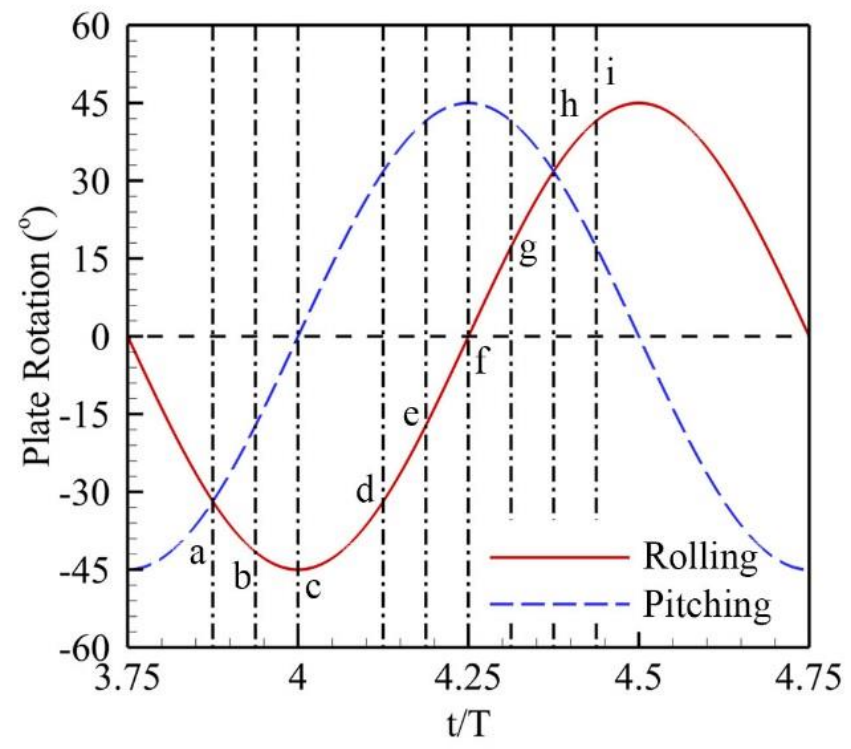

Figure 4-2. The time histories of plate rotation during an entire cycle. The black dash-dot line denotes six moments at $\mathrm{t} / \mathrm{T}=3.875,3.9375,4.0,4.125,4.1875,4.25,4.3125,4.375$, and 4.4375, respectively, for the illustration of the "double C" loop formation during a half cycle in Figure 4-6.

The rolling motion of the plate with respect to the $\mathrm{x}$-axis is given by:

$$
\phi(t)=-A_{\phi} \cos (2 \pi f t)
$$

where $\phi(t)$ is the instantaneous rolling position at time $t, A_{\phi}$ is the rolling amplitude, and $f$ is the flapping frequency. The negative sign of the equation indicates that the rolling motion of the plate begins at the lowest position of a stroke.

The pitching motion with respect to spanwise axis is defined as: 


$$
\theta(t)=-A_{\theta} \cos (2 \pi f t+\psi)+\theta_{\text {bias }}
$$

where $\theta(t)$ is the instantaneous pitching position, $A_{\theta}$ is the pitching amplitude, $\psi$ is the phase difference angle between pitching and rolling motion $\left(\psi=90^{\circ}\right.$ for the baseline case), and $\theta_{\text {bias }}$ is the static pitching bias angle, which is set as $0^{\circ}$ in the current study for generating zero mean lift.

The kinematic parameters adopted in this work are a reasonable representation of the rotation of a low-aspect-ratio insect wings and fish pectoral fins. Table 4-1 provides a concise summary of all the parameters involved and their ranges. The time history of the plate rotational angle is shown in Figure 4-2.

Table 4-1. Parameters involved in the current study and their ranges.

\begin{tabular}{ccccccc}
\hline \hline AR & Re & St & $A_{\phi}$ & $A_{\theta}$ & $\psi$ & $\theta_{\text {bias }}$ \\
\hline $1.27,1.91$, & 100,200, & $0.4,0.6,0.8$, & $45^{\circ}$ & $45^{\circ}$ & $60^{\circ}, 70^{\circ}, 80^{\circ}$, & $0^{\circ}$ \\
2.55 & 400 & $1.0,1.2$ & & & $90^{\circ}, 100^{\circ}, 110^{\circ}, 120^{\circ}$ & \\
\hline \hline
\end{tabular}

\subsection{Computational Setup}

In the current study, the Reynolds number is defined as $R e=U_{\infty} c / v$, based on the incoming flow velocity $\left(U_{\infty}\right)$ and the chord length ( $c$ ). The Strouhal number is defined as $S t=2 A_{\phi} R_{\text {avg }} f / U_{\infty}$, where $A_{\phi}$ is the rolling amplitude and $R_{\text {avg }}$ is the average rotational radius ( $R_{\text {avg }}=S / 2$ ) in spanwise direction. This definition is consistent with previous experimental studies $[30,100]$. 


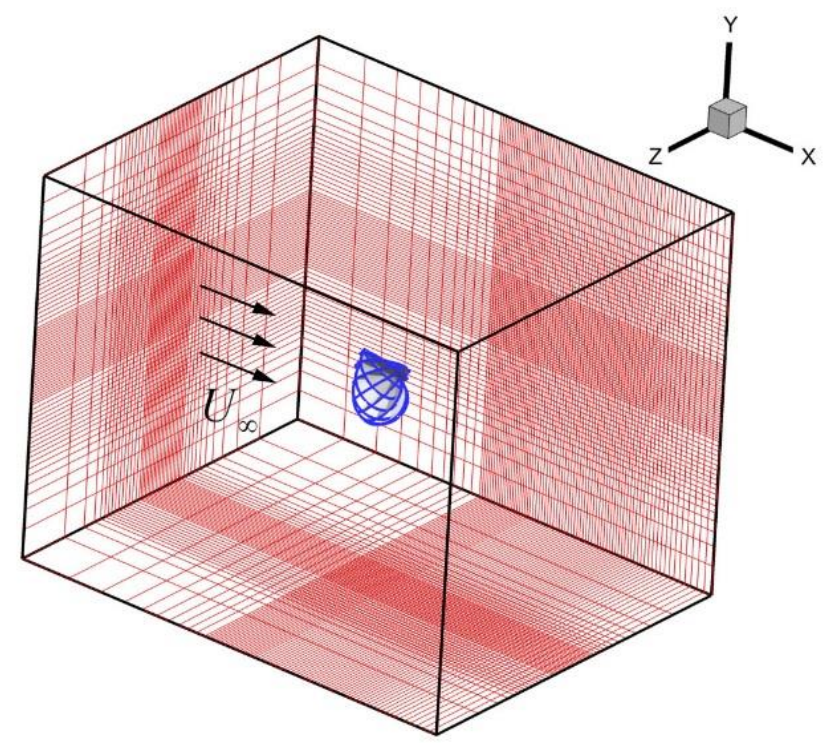

Figure 4-3. Typical grid employed in the current simulations for the circular plate with $A R=1.27$ $(S / c=1.0)$. The grid is $235 \times 233 \times 113$, and the domain size is $30 c \times 25 c \times 25 c$.

The grids employed in the current study are designed to provide high resolution in the region around the flapping plates as well as in the wake region, as shown in Figure 4-3. At the left-hand boundary, we provide a constant inflow velocity boundary condition. The right-hand boundary is the outflow boundary, which is provided with a zero streamwise gradient boundary condition for the velocity, allowing the vortices to convect out of this boundary without significant reflections. The zero-stress boundary condition is provided at all lateral boundaries. A homogeneous Neumann boundary condition is used for the pressure at all boundaries.

To quantify the hydrodynamic performance, the forces and power coefficients are defined as: 


$$
C_{T, L, Z}=\frac{(T, L, Z)}{0.5 \rho U_{\infty}^{2} A_{\text {plan }}} \quad C_{P W}=\frac{P}{0.5 \rho U_{\infty}^{3} A_{\text {plan }}}
$$

where $T, L, Z$ and $P$ are the thrust, lift, spanwise force and hydrodynamic power, respectively, and $A_{\text {plan }}$, the planform area of the plate, is equal to $\pi c S / 4$. The instantaneous hydrodynamic power $(P)$ is defined as the surface integration of the inner product between the pressure and the velocity in each discretized element.

Moreover, the propulsive efficiency is defined as

$$
\eta=\frac{\bar{T} U_{\infty}}{\bar{P}}
$$

where $\bar{T}$ is the cycle-averaged thrust and $\bar{P}$ is the cycle-averaged hydrodynamic power, in which only the positive power is considered.

The computational domain size of all simulations was chosen as $30 c \times 25 c \times 25 c$. This choice was based on our experience with the simulation of such flows and test simulations on a number of different domain sizes. To maintain consistent grid resolutions in both the $y$ - and $\mathrm{z}$ directions, the nominal grid size employed in the current simulations ranged from $235 \times 233 \times 113$ for the smaller aspect-ratio plate $(A R=1.27)$ to $235 \times 273 \times 145$ for the larger aspect-ratio plate $(A R=2.55)$. Figure $4-3$ shows a typical grid used in the current study for the small-aspect-ratio case. As observed in this figure, non-uniform grids with two layers of refined meshes were used in the current study. Very dense meshes were used in a cuboidal region around the plate in all three directions, with the smallest resolution being $\Delta x=0.03 c$. Outside of this region, a layer with slightly coarser meshes $(\Delta x=0.05 c$ ) was arranged in all three directions to resolve the complex wake structures behind the plates. Beyond this layer, the grid is rapidly 
stretched in the $y$ - and $\mathrm{z}$-directions. In the $\mathrm{x}$-direction, the stretching is rapid upstream of the plate, where we do not expect any streamwise gradients. In the wake region, however, the stretching factor was maintained at $3 \%$ to ensure a relatively high streamwise resolution. This arrangement can limit the numerical dispersion that is associated with the use of central difference schemes on highly stretched meshes [101].

Comprehensive studies have been conducted to assess the effect of the spatial and temporal grid resolution on the salient features of the computed flow and to demonstrate that the chosen grids produce accurate results. Spatial grid refinement studies were conducted by simultaneously doubling the grid in all three dimensions in the refined zones. The overall grid size for this refined grid was $283 \times 273 \times 169$ (approximately 13.1 million grid points). Temporal independence studies were conducted by halving the $\Delta t$ of the original case while using the same nominal grids. All these simulations were conducted on a Xeon E5-2609 CPU with 128 GB of core memory, and each nominal grid simulation required anywhere from 96 to 120 CPU hours.

Table 4-2. Results of spatial and temporal grid independence study for $A R=1.27, S t=0.6$ and

$$
R e=200 \text { case. }
$$

\begin{tabular}{ccccc}
\hline \hline Case & $\bar{C}_{T}$ & $\left(C_{L}\right)_{\text {r.m.s. }}$ & $\left(C_{Z}\right)_{\text {r.m.s. }}$ & $\eta$ \\
\hline Nominal grid & 1.462 & 3.417 & 1.912 & 0.1927 \\
Finer spatial grid & 1.459 & 3.375 & 1.897 & 0.1921 \\
Finer temporal step & 1.461 & 3.409 & 1.905 & 0.1925 \\
\hline \hline
\end{tabular}


Table 4-2 compares the key hydrodynamic quantities for the spatial and temporal grid independence studies conducted for the $A R=1.27, S t=0.6$ and $R e=200$ case. The table shows that the spatial and temporal grid refinement leads to a difference in the mean thrust and efficiency of less than $1 \%$ and a variation in the root mean square (r.m.s.) lift and spanwise force of approximately $2 \%$ at most. This clearly demonstrates that the hydrodynamic forces computed in the current study were grid independent. In addition to the above comparison of the hydrodynamic forces and efficiency, it is useful to assess the effect of the grid on the flow development in the wake. In Figure 4-4, we compare the wake profiles for these three different grids. Figure 4-4 (a) and (b) show the mean streamwise $\left(\bar{u}_{1}-U_{\infty}\right)$ and transverse $\left(\bar{u}_{2}\right)$ velocity profiles at the mid-span, and Figure 4-4 (c) shows the spanwise mean velocity profiles at the center of the XZ-plane. In all of these plots, we note that the differences between the profiles computed using the three different grids are negligible. Also plotted in Figure 4-4 (d) are profiles of the fluctuation kinetic energy, defined as $\left(\overline{u_{1}^{\prime 2}}+\overline{u_{2}^{\prime 2}}+\overline{u_{3}^{\prime 2}}\right) / 2$, in the mid-span plane. A comparison of these profiles is an even more aggressive test of grid dependence because the fluctuations inherently contain more information from the smaller spatial and temporal scales in the flow. The difference between the three sets of profiles is small, which clearly establishes the fidelity and accuracy of the current simulations. 
(a)

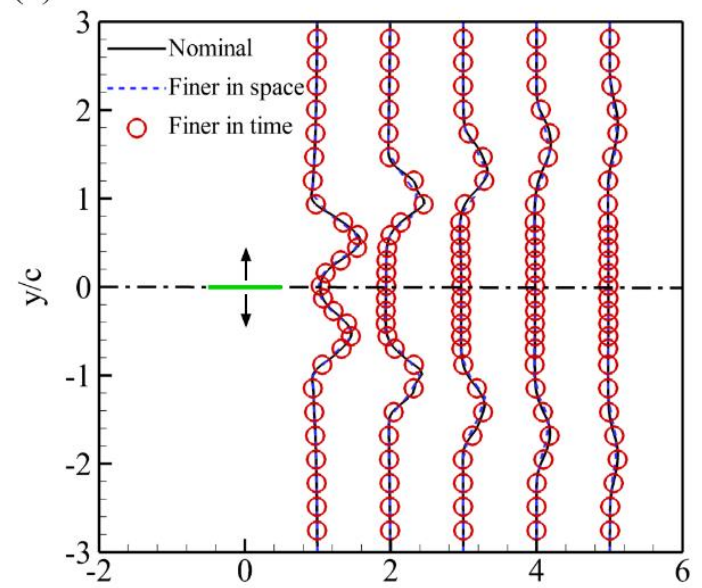

(c)

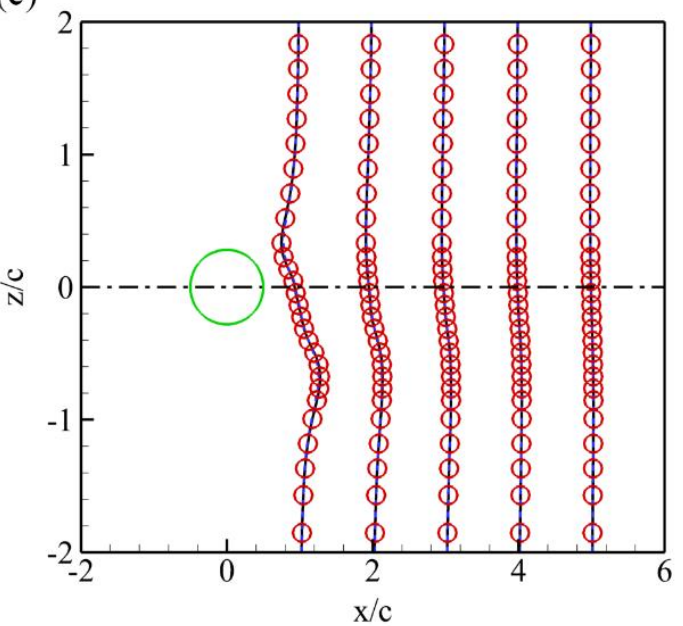

(b)

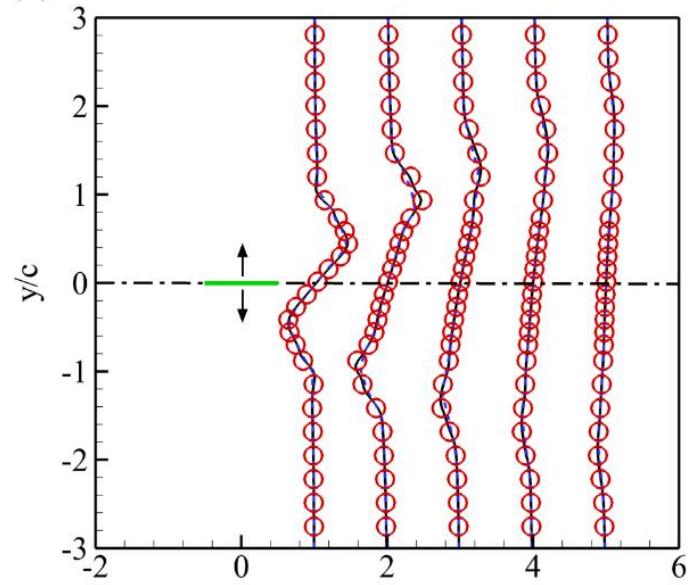

(d)

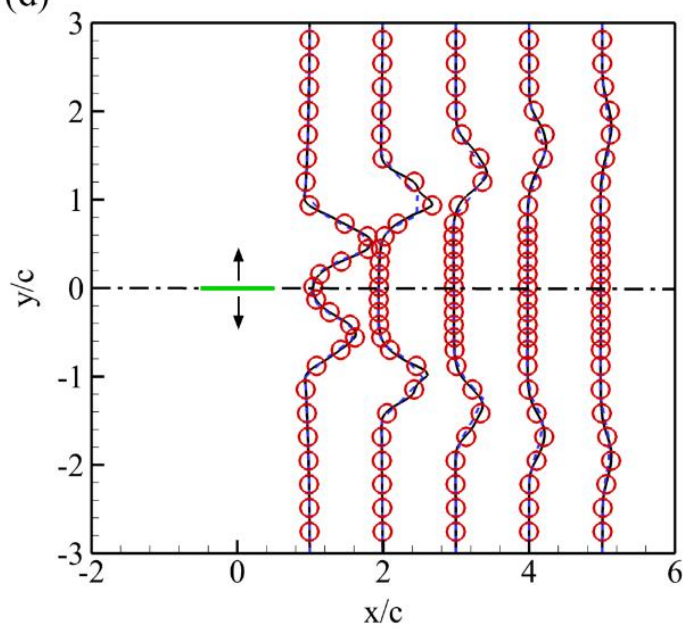

Figure 4-4. Comparison of computed profiles in the wake demonstrating the grid independence of the computed results for the $A R=1.27, S t=0.6$ and $R e=200$ case. Five profiles at $\mathrm{x} / \mathrm{c}=1,2,3,4$ and 5 are shown. (a) Streamwise $\left(\bar{u}_{1}-U_{\infty}\right)$ velocity profiles. (b) Transverse $\left(\bar{u}_{2}\right)$ velocity profiles. (c) Spanwise $\left(\bar{u}_{3}\right)$ velocity profiles. (d) Fluctuation kinetic energy $\left(\overline{u_{1}^{\prime 2}}+\overline{u_{2}^{\prime 2}}+\overline{u_{3}^{\prime 2}}\right) / 2$ at the center of the XY-plane.

To better evaluate the effects of different grids on the instantaneous vortical structure, we also quantified the size of vortex loop by measuring the slides of 3-D wake topology in both near 
and far wake at $\mathrm{t} / \mathrm{T}=4.25$, as shown in Figure 4-5. Specifically, the distances between each pair of vortices were measured. These distances represent the diameters of the vortex loops formed in the downstream of the plate. Detailed the discussion of the 3-D vortex loop structures can be found in FIG. 8.
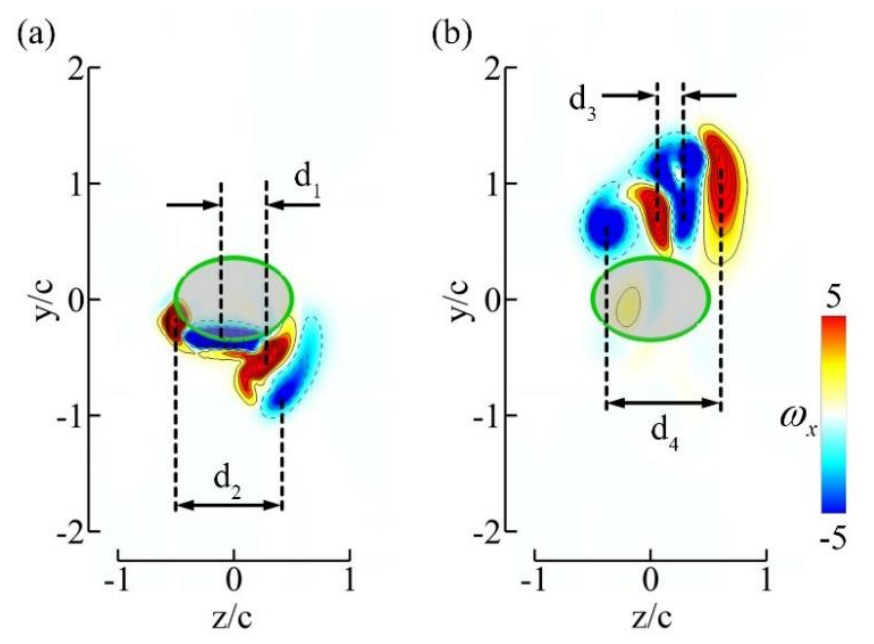

Figure 4-5. Slides of $2-\mathrm{D}$ contours of streamwise vorticity at $\mathrm{t} / \mathrm{T}=4.25$ in the near wake (a) and far wake (b), respectively, for measuring the relative changes of vortical structure at different spatial and temporal grid. The corresponding section-cutting locations can be found in Figure 4-8 (c) and (f).

As shown in Figure 4-5 (a), the distances between the inner vortex pair and the outer vortex pair are denoted as $d_{1}$ and $d_{2}$, respectively, in which the center of each vortex is chosen as the point with highest value of streamwise vorticity $\left(\omega_{x}\right)$. Similarly, in Figure 4-5 (b), the distances between another two vortex pairs shed during the previous half stroke are denoted as $\mathrm{d}_{3}$ and $\mathrm{d}_{4}$, respectively. Table 4-3 compares these distance quantities for the spatial and temporal grid 
independence studies conducted for the $A R=1.27, S t=0.6$ and $R e=200$ case. The table shows that the spatial and temporal grid refinement leads to a difference in the near wake vortices distances less than $2 \%$ and a variation in the far wake vortices distances of approximately $4 \%$ at most. This clearly indicates that the vortical structures computed using current set up were also grid independent.

Table 4-3. Comparison of computed distances of vortex paired in the near wake and far wake between different grid resolutions.

\begin{tabular}{ccccc}
\hline \hline Case & $\mathrm{d}_{1}$ & $\mathrm{~d}_{2}$ & $\mathrm{~d}_{3}$ & $\mathrm{~d}_{4}$ \\
\hline Nominal grid & 0.438 & 0.875 & 0.219 & 0.981 \\
Finer spatial grid & 0.430 & 0.867 & 0.211 & 0.969 \\
Finer temporal step & 0.433 & 0.871 & 0.214 & 0.977 \\
\hline \hline
\end{tabular}

\subsection{Wake Topology and Propulsive Performance}

In this section, we first present a detailed discussion of the wake topology as well as the force production of a circular plate undergoing a pitching-rolling motion. Following this discussion, the effect of the Strouhal number, Reynolds number, aspect ratio, and phase difference angle on the vortex dynamics and associated propulsive performance are discussed for the range of parameters listed in Table 4-1. 


\subsubsection{Baseline Case}

The wake topology and force production for the flapping circular plate is examined at $S t=0.6$ and $R e=200$ with $\psi=90^{\circ}$ in this section. Figure 4-6 shows the vortex shedding process near the plate trailing edge at nine phases. The corresponding plate kinematics can be found in Figure 4-2. The shell and core of the vortex structures are visualized by the Q-criterion [94] with two iso-surface values, $\mathrm{Q}=1$ (in grey) and 6 (in color), respectively. The vortex cores are colored according to $\omega_{x}$ to indicate the vorticity convection direction. The senses of the vorticity in each section are indicated by arrows according to the right-hand rule.

As the plate rolling downward from the middle point to the lowest point (Figure 4-6 (a-c)), a "C"-shaped vortex loop is found formed by the root vortex $\left(V_{1}\right)$, the tip vortex $\left(V_{2}\right)$ and the trailing-edge vortex (TEV). Note that the rolling motion creates different shear rates between the plate root and tip and affects the development of the vortex loop. The strength asymmetry between $V_{1}$ and $V_{2}$ can be easily observed in Figure 4-6 (c) when the plate reaches to the lowest point. When the tip of plate starts rolling upward from the lowest point to the middle point of the cycle, the leading-edge of the plate is also pitching up (as shown in Figure 4-6 (d-f)). During this period, newly developed vortices, resulting from the trailing-edge shear layer $\left(V_{3}\right)$ and the leading-edge vortex $\left(V_{4}\right)$, come together and form a new inner "C"-shaped vortex loop with an opposite sense to the outer loop $\left(V_{1}\right.$ and $\left.V_{2}\right)$. It is worth noting that the newly formed inner "C"shaped vortex loop is a completely different structure from the contrails generated by the previous vortex loop. 
(a) $\mathrm{x}$

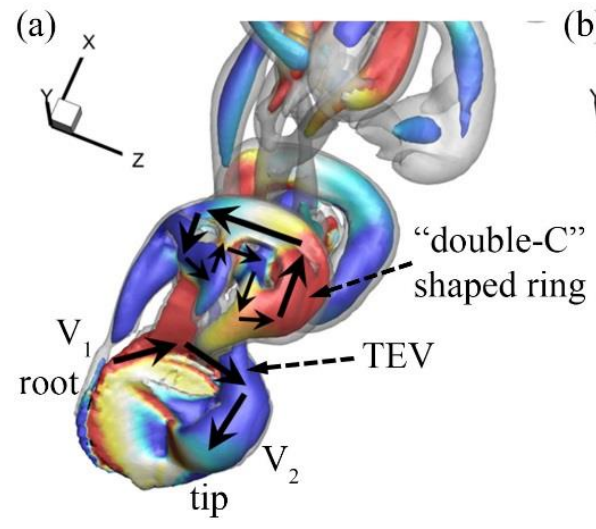

(d)
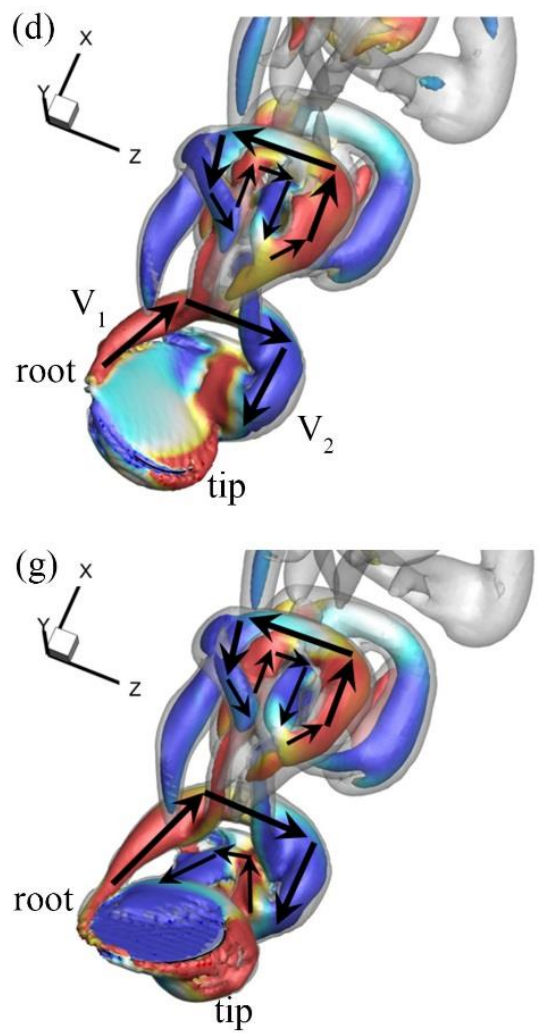

(b)

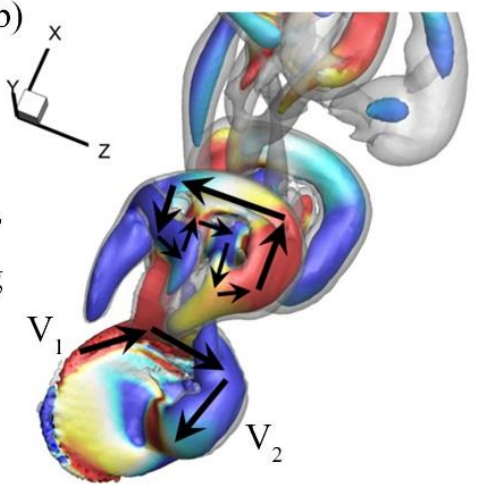

(e) $\mathrm{x}$

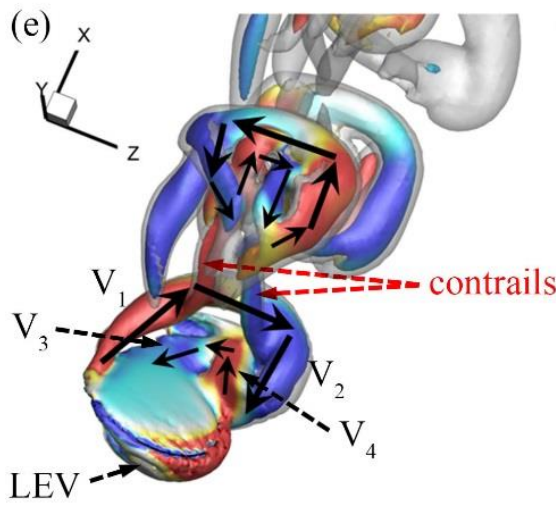

(h)

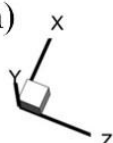

(c)

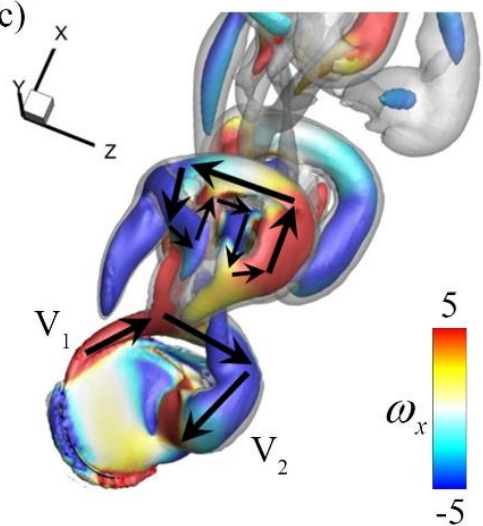

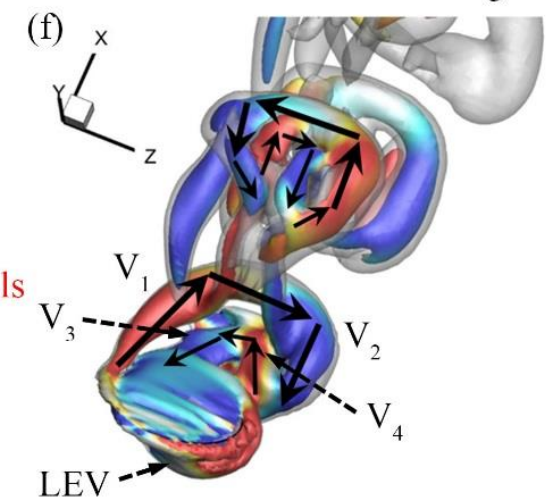

LEV

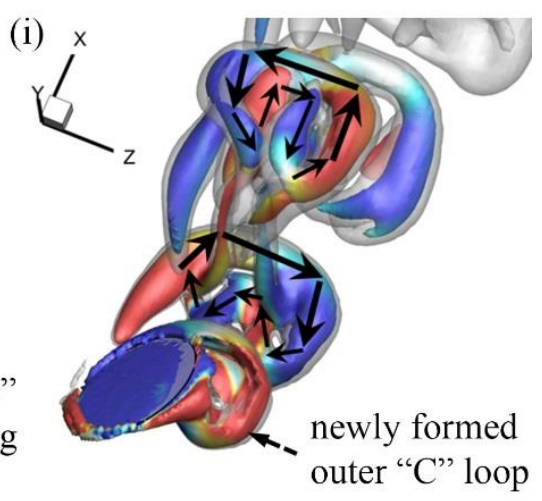

Figure 4-6. The wake topology of a flapping circular plate $(A R=1.27)$ with $S t=0.6$ and $R e=200$ at $\mathrm{t} / \mathrm{T}=3.875,3.9375,4.0,4.125,4.1875,4.25,4.3125,4.375$, and 4.4375 , respectively. In (a-c), the plate is rolling downward and reaches to the lowest point at $\mathrm{t} / \mathrm{T}=4.0$; in $(\mathrm{d}-\mathrm{f})$, the plate is rolling upward and reaches to the middle point at $\mathrm{t} / \mathrm{T}=4.25$; in $(\mathrm{g}-\mathrm{i})$, as the plate continuous rolling upward, the newly formed double-C shaped ring gradually shed our from the plate. The time history of the corresponding rolling and pitching angles are shown in Figure 4-2. The iso-surface 
contours are colored based on the streamwise vorticity $\left(\omega_{x}\right)$.

Note that the "double-C"-shaped vortex structures generated around the plate trailing edge are distinct from the observations in previous studies on purely pitching $[24,25,102,103]$, purely heaving[21], and pitching-heaving[23,26,27] panels/foils. In those previous studies, only single interconnected vortex loops were observed around the trailing edge during each half cycle, and the loops exhibited symmetry about the spanwise central plane $[21,27]$. In the current study, however, the rolling motion results in a faster development of the leading-edge vortex $\left(V_{3}\right)$. By comparing Figure 4-6 (c) and (f), it can be observed that a strong leading-edge vortex is formed within only one quarter cycle and is then stretched and tilted along the tip connecting to the trailing-edge shear layer $\left(V_{4}\right)$. This flow feature distinguishes the current wake structures from those in the previous pitching and/or heaving studies.

Along with the plate continually moving upward, both " $\mathrm{C}$ "-shaped vortex loops started to shed outward from the plate trailing edge and connected with each other, as shown in Figure 4-6 (g-i). As a result, a "double-C"-shaped vortex ring was formed and convected downstream. At the same time, a new outer "C"-shaped vortex loop starts to form. During the second half cycle, another "double-C"-shaped vortex ring (as shown in Figure 4-6 (a), which was formed a cycle earlier) with the opposite sense formed in the same manner. A pair of "double-C"-shaped vortex rings were then produced from each flapping cycle and resulted in a bifurcated wake pattern in the downstream (Figure 4-7 (a)). The side view (Figure 4-7 (b)) of the bifurcating wake pattern is similar to that observed in previous studies on pitching and/or heaving plates $[21,25,26]$. However, according to the top view (Figure 4-7 (c)), two key features differentiate the current 
wake structures from those of previous studies. First, in previous pitching and/or heaving studies $[21,25,26]$, the vorticity generated by the streamwise edges dominates the evolution of the wake. The stronger tip vortices induce a compression on the associated spanwise vortices. As the vortex rings convect downstream, a spanwise narrowing of the wake can be observed from the top view (i.e., Fig. 16 in Ref.[25] and Fig. 9 in Ref.[26]). However, a similar narrowing process was not found in the downstream wake of the present work. Instead, the size of the vortex rings gradually increased as they convected downstream, as can be observed in Figure 4-7 (c) by comparing the size of the shed vortex rings $R_{1}, R_{3}, R_{5}$, and $R_{7}$ in the formation sequence. This difference can be explained by the increased spanwise flow caused by the rolling motion. For the pitching-rolling plate, the intensification of the leading-edge vortex and the diminishment of the root vortex attenuate the compression conditions found in the pitching-heaving plates[26] and result in the elongation of the vortex rings. Second, as the vortex rings convect downstream, a slight wake deflection in the spanwise direction can be observed from the top view of the wake topology (Figure 4-7 (c)). Furthermore, note that there are two sets of vortex 'contrails' (see, for instance, rings in Figure 4-7 (b)) at its upstream and downstream ends that extend toward its two adjacent rings. As the vortices convect downstream, these vortex contrails become weaker and gradually annihilate. Simultaneously, the "double-C"-shaped vortex rings gradually evolve into single-loop vortex rings (see, for instance, ring $R_{3}$ in Figure 4-7).

To better understand the structure of the "double-C"-shaped vortex ring and the interaction between two adjacent vortex rings, several YZ-plane slices were cut into the wake when the plate was at the middle of its upstroke $(\mathrm{t} / \mathrm{T}=4.25)$, and the streamwise vorticity contours $\left(\omega_{x}\right)$ are plotted in Figure 4-8. Red and blue indicate the counter-clockwise and clockwise rotation directions, respectively. 
(a)

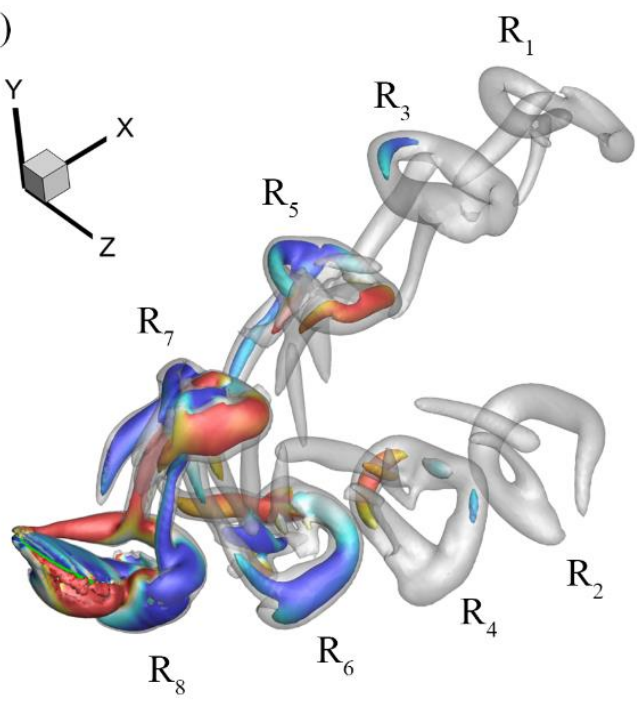

(b)

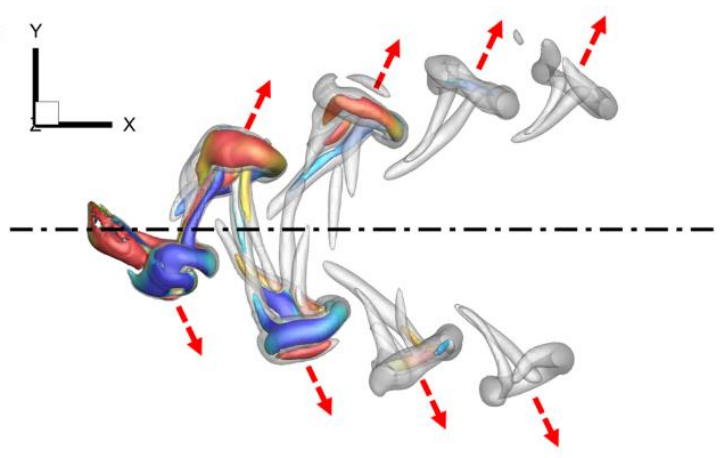

(c)

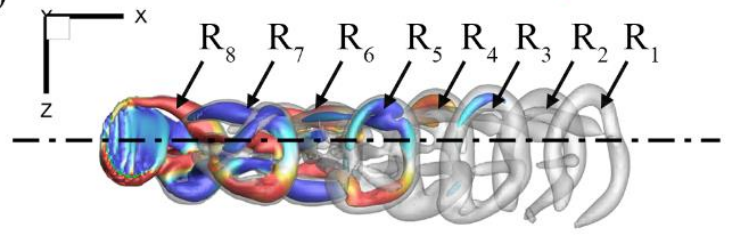

Figure 4-7. Wake topology of a pitching-rolling circular plate $(A R=1.27)$ after four flapping cycles. The plate is at the phase where it is in the center position of its rolling motion and about to move upward (t/T=4.25). (a) Perspective view, (b) side view, and (c) top view. The vortex loops are labeled based on their shedding sequence, and the propagation directions of the rings are indicated by red arrows in (b).

In the 2-D contours of Figure 4-8, the contained vortices of the "double-C"-shaped rings $R_{8}$ and $R_{7}$ are labeled as $V_{1} \sim V_{4}$ and $V_{5} \sim V_{8}$, respectively. Figure 4-8 (a-d) show the detailed structures of the inner and outer " $\mathrm{C}$ " loops of $R_{8}$, in which Figure 4-8 (a-c) are at the upstream of the trailing edge and Figure 4-8 (d) is slightly behind the plate trailing edge. Figure 4-8 (e-h) illustrate the vortical structure of the "double-C"-shaped ring $R_{7}$, which was shed from the previous half cycle with the opposite sense of $R_{8}$. Figure 4-8 (e-f) show the interaction between $R_{7}$ and $R_{8}$. It can be observed that the outer "C" loop $\left(V_{1}\right.$ and $\left.V_{2}\right)$ of $R_{8}$ is connected with the 
inner "C" loop ( $V_{7}$ and $V_{8}$ ) of $R_{7}$ by a pair of contrails in the upstream. The interaction between $R_{7}$ and $R_{6}$ can also be observed in Figure 4-8 $(\mathrm{g}-\mathrm{h})$, where another pair of contrails with alternating signs is in the downstream between the outer "C" loop of $R_{7}$ and the inner "C" loop of $R_{6}$. The wake pattern begins to repeat in the far wake (Figure 4-8 (i)). Furthermore, as shown in Figure 4-8 (j-1), due to the viscous dissipation effect at such a low Reynolds number, the "double-C" vortical structures gradually evolved into single-loop rings.

Another distinct feature that can be observed in Figure 4-8 is that the strengths of the vortex cores for both the inner and outer "C" loops are inequivalent. This is because the rolling motion of the plate created a difference in the shear rate between the plate root and tip. As the plate rolled either upward or downward, the formed vortex loops were strengthened near the plate tip and weakened about the root and thus led to an asymmetric ring shape. As a consequence, the downstream wake pattern is found slight defected from the midline in the span towards the plate tip, as shown in Figure 4-7 (c). 


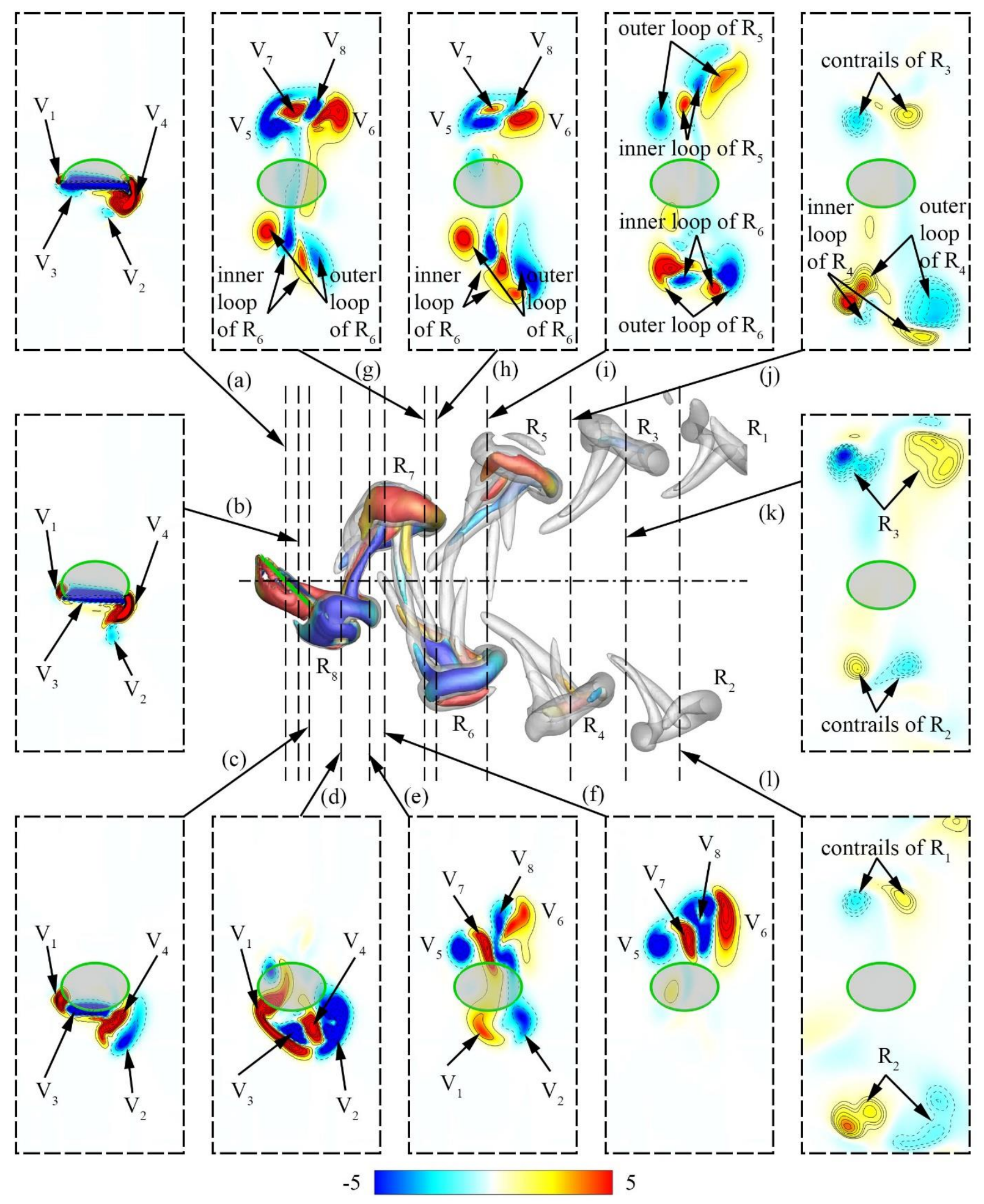

Figure 4-8. Slides of the contours of streamwise vorticity $\left(\omega_{x}\right)$ at $t / T=4.25$. (a-d) the vortical 
structure of $R_{8}$, which consists of vortex $V_{1} \sim V_{4}$. (e-f) the vortical structure of $R_{7}$, which consists of vortex $V_{5} \sim V_{8}$. The vortex interactions occurring between $R_{7}$ and $R_{6}$ are shown in (g-h), and the interactions between $R_{5}$ and $R_{6}$ are presented in (i). In the far wake, the double-Cshaped ring gradually evolves into a single-loop vortex $(\mathrm{j}-1)$.

Figure 4-9 shows the temporal variation in the force coefficients over two cycles when the forces reached a periodic stage. To provide a better understanding of vortex forming/shedding process, the superimposed pitching and rolling motion curves are also included in Figure 4-9. The cycle-averaged value of each force component is indicated by the dash-dotted line. In Figure 4-9 (a), the thrust peaks occurred twice during each cycle at the instant when the plate was near the center of its trajectory. A slight drag was produced when the plate started to reverse its rolling direction. The maximum and mean thrust coefficients for this case were found to be 3.98 and 1.46, respectively. Figure 4-9 (b) and (c) show the other two force components produced by the plate. Both forces have equivalent positive and negative variations over a cycle. The calculated mean lift and spanwise forces are 0.0 and 0.1 , respectively. Note that the peak values of the lift $\left(\left|C_{L}\right|_{\max }=5.35\right)$ and spanwise force $\left(\left|C_{Z}\right|_{\max }=3.19\right)$ components are in a similar range to the maximum peak thrust. This is different from the findings of previous pitching-heaving studies $[26,27,28]$, in which the peak thrust is significantly smaller than the peak lift. In addition, two peaks of the thrust and lift production can be observed during each half stroke. The first peak has a smaller magnitude than the second one. This phenomenon could be related to the doubleloop vortex shedding process close to the plate trailing edge. It is worth noting that the double peak in force production observed in the pitching-rolling plates is in line with previous studies on 
bio-inspired flapping wings/fins, which include experimental measurements of insect wings[104,105] and numerical simulations of insect model wings[106,107] and fish pectoral fins[108,109].
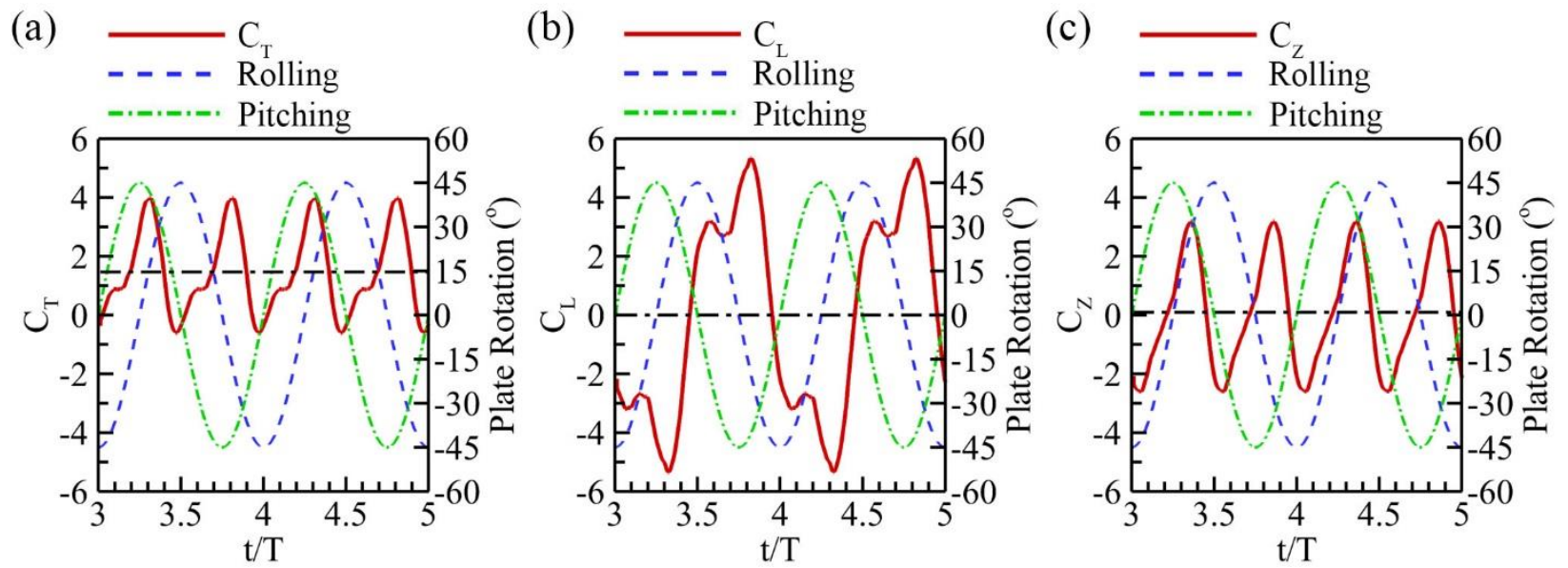

Figure 4-9. Computed time-varying force coefficients for the circular plate $(A R=1.27)$ at $R e=200$ and $S t=0.6$. (a) Thrust coefficient, (b) lift coefficient, and (c) spanwise force coefficient. The black dash-dotted lines indicate the corresponding cycle-averaged value. The pitching and rolling motion curves of the plate are also superimposed with the force history.

\subsubsection{Effects of Strouhal Number}

In this section, we examined the effect of the Strouhal number on the wake topology and propulsive performance of the circular plate $(A R=1.27)$ at $R e=200$ and $\psi=90^{\circ}$. Figure $4-10$ presents both side and top views of the wake topology for $S t=0.4$ and 0.8 , respectively. This plot can be examined in conjunction with the same plots for the $S t=0.6$ case in Figure 4-7. 
(a)

(b)

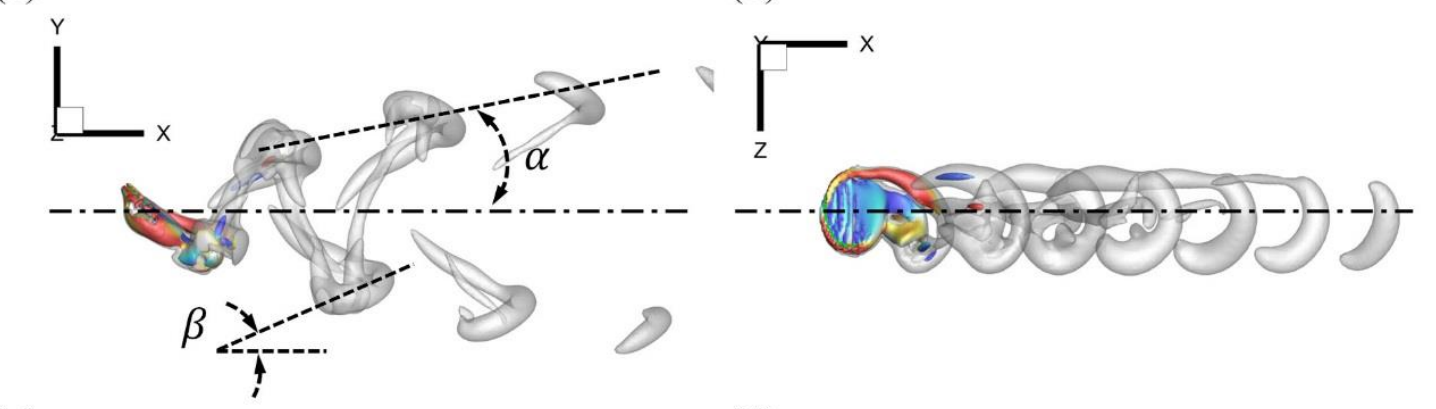

(c)

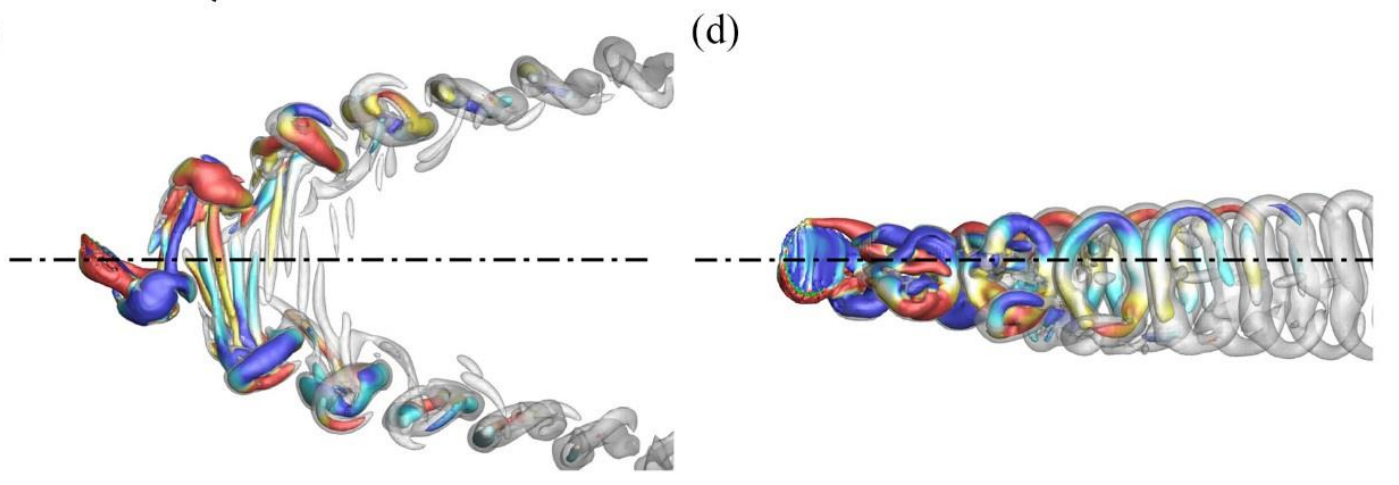

Figure 4-10. Wake topology for the $A R=1.27$ circular plate during the phase where the plate is at the middle point in its up stroke. The left and right columns show the side view and top view, respectively. (a-b) The side view and top view of the case of $S t=0.4$. (c-d) The side view and top view of the case for $S t=0.8$. The Reynolds number is 200 for all these cases.

As shown in Figure 4-10 (a-b), the lower Strouhal number case $(S t=0.4)$ shows a decreased vorticity strength. In this case, the "double-C"-shaped vortex rings rapidly evolved to single vortex rings. In contrast, the higher Strouhal number case $(S t=0.8)$ presents a delay process for this wake evolution, as shown in Figure 4-10 (c-d). In addition, the concomitant increase in vorticity strength led to the enhancement of the mutual induction between two adjacent vortex rings. As a consequence, the oblique angle $(\alpha)$ between two sets of vortex rings increased with increasing Strouhal number, which can be observed by comparing Figure 4-10 (a, c) with Figure 
4-7 (b). This change in oblique angle can affect the efficiency of momentum transport in the downstream, implying the existence of an optimal Strouhal number in terms of propulsive efficiency. To further quantify the inclination angle of the vortex formation, both the wake oblique angle $(\alpha)$ and the vortex ring orientation angle $(\beta)$ with respect to the wake centerline were measured in the near wake. As tabulated in Table 4-4, the oblique angle of the bifurcated wake increases monotonically with the Strouhal number. On the other hand, the angle of the vortex ring orientation initially follows an increasing trend, reaches a maximum value at $S t=1.0$, and then decreases abruptly. As the shed vortex ring convecting downstream, the strength of the vortex ring is gradually decreasing due to the viscous dissipation effect. As a result, both the oblique angle and vortex ring orientation angle are reduced along the downstream direction. This phenomenon is especially clear for the high Strouhal number cases, i.e. at $S t=0.8$ (Figure 4-10 (cd)).

Another salient feature that must be noted here is the wake deflection along the mid-span, which can be easily observed in Figure 4-10 (b, d) and Figure 4-7 (c). For lower Strouhal number cases (i.e., $S t=0.4$ and 0.6), the wake always deflect towards to the plate tip direction. For the higher Strouhal number case $(S t=0.8)$, however, the wake initially defects to the tip direction in the near wake, and then gradually deflects back in the far wake. A higher self-induced vortex ring velocity for the higher Strouhal number cases might be responsible for this wake deflection phenomenon. In addition, the viscous effects in the far wake could also play a role for the wake evolution. Furthermore, as the vortex rings convecting downstream, the elongation of the vortex rings is proportional to the increment of the Strouhal number. 


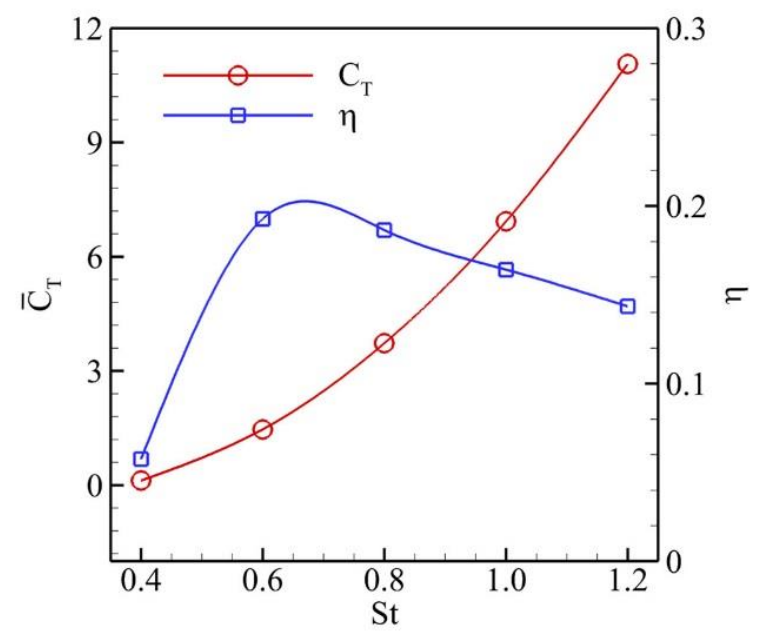

Figure 4-11. Variation in cycle-averaged hydrodynamic performance for the baseline case with changing Strouhal number. The red and blue curves indicate the mean thrust coefficient and propulsive efficiency, respectively.

Table 4-4. Comparison of the wake oblique angle $(\alpha)$ and vortex ring orientation angle $(\beta)$.

\begin{tabular}{ccc}
\hline \hline$S t$ & $\alpha\left(^{\circ}\right)$ & $\beta\left(^{\circ}\right)$ \\
\hline 0.4 & 16 & 20 \\
0.6 & 21 & 24 \\
0.8 & 27 & 35 \\
1.0 & 30 & 40 \\
1.2 & 31 & 28 \\
\hline \hline
\end{tabular}

Figure 4-11 shows the variation in the mean thrust coefficient and efficiency as a function of Strouhal number for the baseline case. First, it can be observed from this figure that for all cases, the thrust monotonically increases with the Strouhal number $(0.4 \leq S t \leq 1.2)$. Such 
behavior has been well documented for both two-dimensional $[8,9]$ and low-aspect-ratio $[25,26]$ pitching and/or heaving foils. In addition, the efficiency exhibits an initial rapid increase and then gradually decreases with increasing Strouhal number. The maximum value of the efficiency is reached at $S t=0.6$, which is higher than the range usually considered optimal for swimming and flying animals $[110,111]$. This is probably due to the particular choice of parameters, such as the maximum pitching angle and Reynolds number, in the current study. The relatively low Reynolds number in the current study leads to a proportionately large shear drag that has to be overcome for the plate to produce a net thrust. This tends to push the optimal Strouhal numbers to higher values. As shown in Ref.[112], even at high Reynolds number, depending on the maximum angle of attack, the optimal Strouhal numbers can vary from 0.3 to 0.6 for a rigid flapping foil.

\subsubsection{Effects of Reynolds Number}

In this section, two additional simulations, one at $R e=100$ and the other at 400 , have been conducted for the baseline case to assess the effect of the Reynolds number on the wake topology and instantaneous force generation. Note that the higher Reynolds number case was conducted on the finer spatial meshes.

Figure 4-12 shows a perspective view of the vortex topology for these cases, and this can be compared with the corresponding plot in Figure 4-6 (f). For the lower Reynolds number of 100, the formation of the double-loop vortex around the plate trailing edge remained evident, although the loops rapidly dissipated after they were shed into the wake. This behavior is consistent with the increased viscous effect in this case. The higher Reynolds number case, on the other hand, 
shows many of the features observed for the case at $R e=200$ in both the near and the far flow field. In particular, the wake was also found to exhibit "double-C"-shaped rings propagating downstream. This indicates the basic vortex dynamics of these low-aspect-ratio flapping foils are insensitive to changes in the Reynolds number between 100 and 400.

(a)

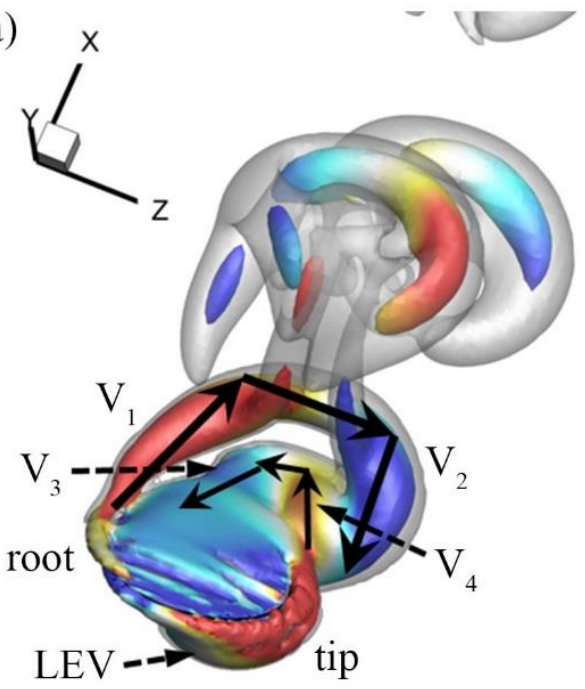

(b)

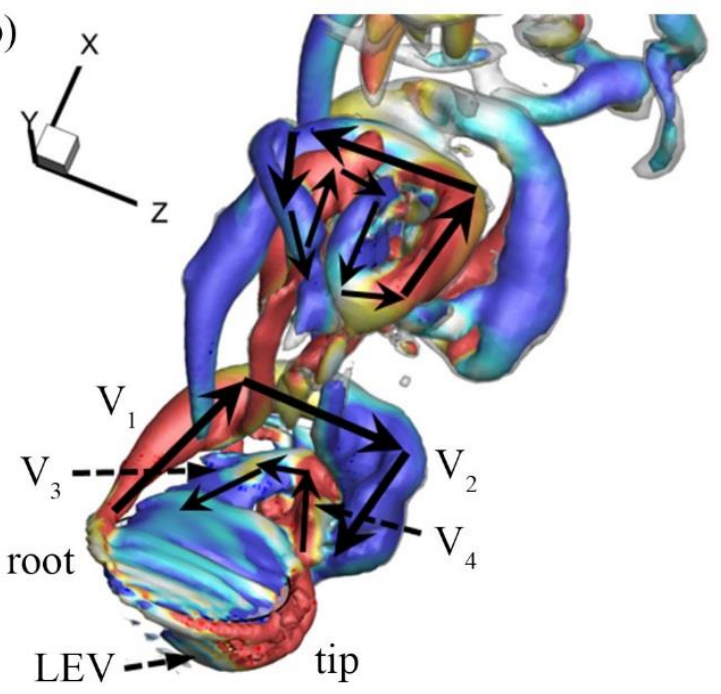

Figure 4-12. Vortex topology of a circular plate $(A R=1.27)$ for different Reynolds numbers. (a) $R e=100$. (b) $R e=400$. The Strouhal number is 0.6 in both cases.

Figure 4-13 shows the time-varying force coefficients of the plate flapping at an aspect ratio of 1.27 for Reynolds numbers ranging between 100 and 400 with $S t=0.6$. The corresponding $R e=200$ case is re-plotted for comparison. As shown in Figure 4-13 (a), the thrust coefficient increases with the Reynolds number. Specially, the peak thrust coefficient for the $R e=100$ case is approximately 3.45 , whereas that for the $R e=400$ case is approximately 4.35 , which amounts to an increase in magnitude of over $26 \%$. The mean thrust coefficients for the $R e=100$ and $R e=400$ case are 1.17 and 1.65, respectively. For the lift coefficient (Figure 4-13 (b)), the double peaks 
appear for all Reynolds numbers but with slight differences in magnitude. In Figure 4-13 (c), the spanwise force exhibits a smaller negative peak with increasing Reynolds number. The mean values of both the lift and spanwise force coefficients are approximately zero.
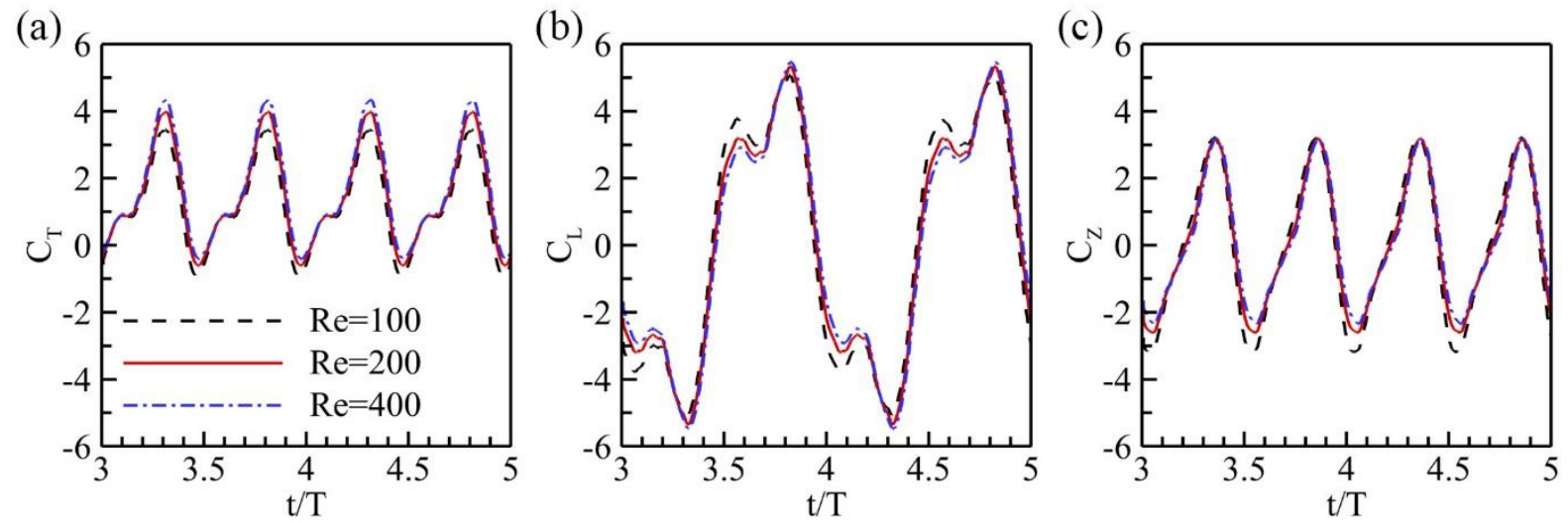

Figure 4-13. Computed time-varying force coefficients for the circular plate $(A R=1.27)$ at various Reynolds numbers. (a) Thrust coefficient, (b) lift coefficient, and (c) spanwise force coefficient. The Strouhal number is 0.6 for all of these cases.

\subsubsection{Effects of Aspect Ratio}

In this section, we examined the effect of the plate aspect ratio $(A R)$ on the wake topology and instantaneous force production. Figure 4-14 shows the vortical structures for the elliptical plate with $A R=1.91$ and 2.55 at $S t=0.6, R e=200$, and $\psi=90^{\circ}$. This plot can be compared with the corresponding plot in Figure 4-6 (f). To illustrate the effect of plate aspect-ratio on the inn-loop development in Figure 4-14, the 2-D streamwise vorticity contours $\left(\omega_{x}\right)$ and spanwise vorticity contours $\left(\omega_{z}\right)$ are shown in Figure 4-15 (a-c) and Figure 4-15 (d-f), respectively. It shows that the size of the inner-loop in streamwise direction (which consists of $V_{3}$ and $V_{4}$ ) increases along 
with the increment of plate aspect-ratios (Figure 4-15 (a-c)), however, the spanwise vorticity magnitude of the inner loop decreases (Figure 4-15 (d-f)).
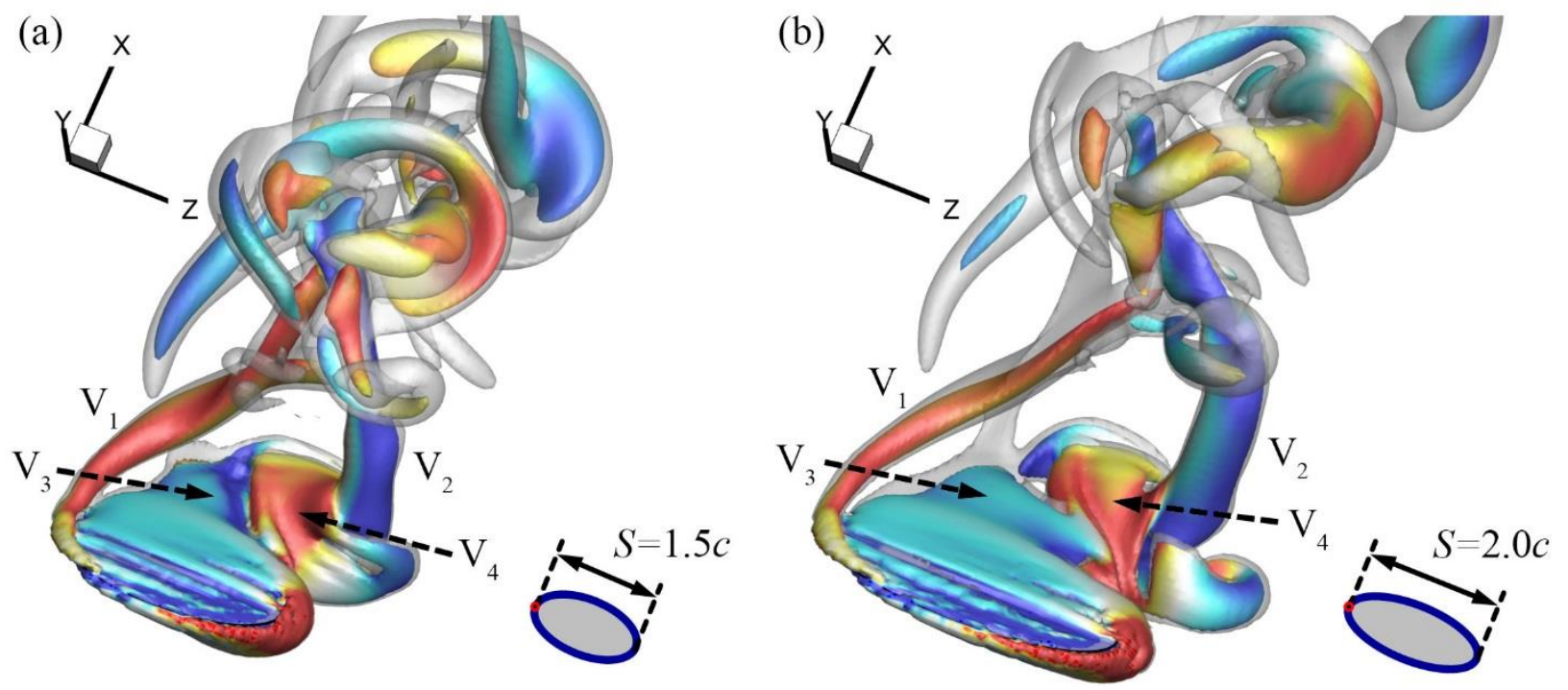

Figure 4-14. Vortex topology for an elliptical plate with $A R=1.91$ (a) and $A R=2.55$ (b). For each case, the Strouhal number and Reynolds number are 0.6 and 200, respectively.

Figure 4-16 shows the time history of the force coefficients for the case of different aspect ratios at $R e=200$ and $S t=0.6$, and a number of interesting observations can be made. First, as the aspect ratio increases, the thrust peak increases and the magnitudes of the other two transverse forces decrease. The mean thrust coefficients for the $A R=1.91$ and $A R=2.55$ case are 1.62 and 1.77, respectively. This is a clear indication of what we expect to be a relatively high propulsive efficiency for a larger aspect-ratio plate. Second, the magnitude of the first force peak gradually decreases with increasing aspect ratio. This can be explained by the vortex formation exhibited by higher aspect-ratio plates. The outer "C" loop becomes weaker with increasing aspect ratio, 
and the inner " $\mathrm{C}$ " loop begins to dominate the vortical structure. Thus, the magnitude of the first force peak gradually decreases.

(a)
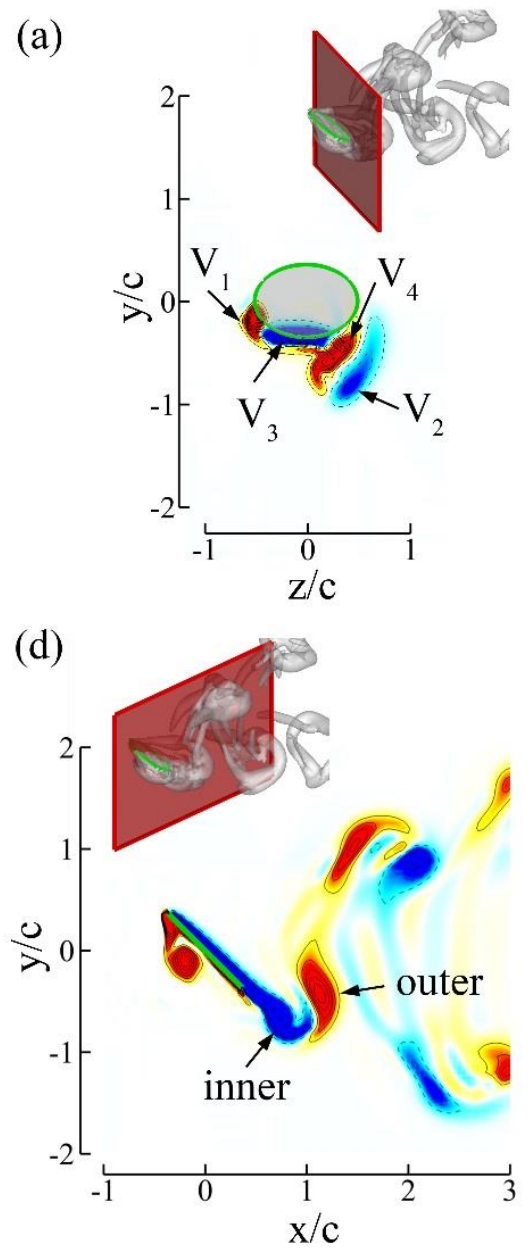

(b)

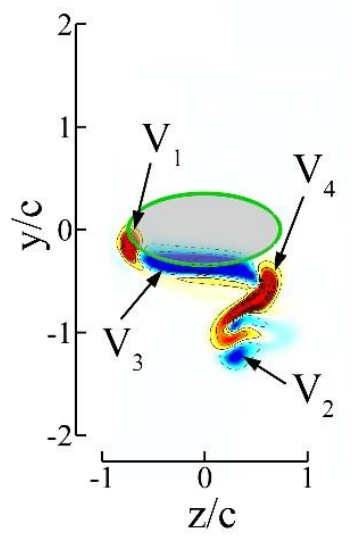

(e)

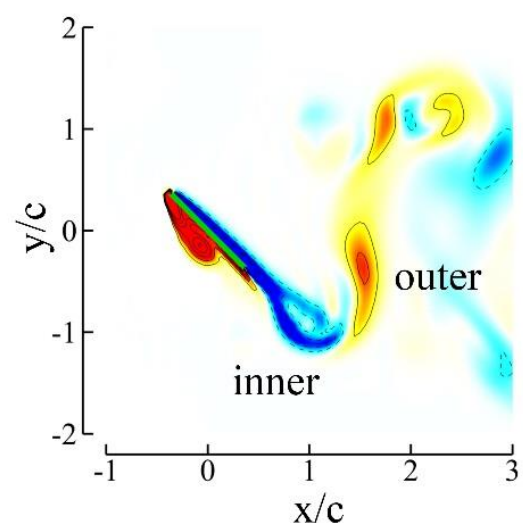

(c)

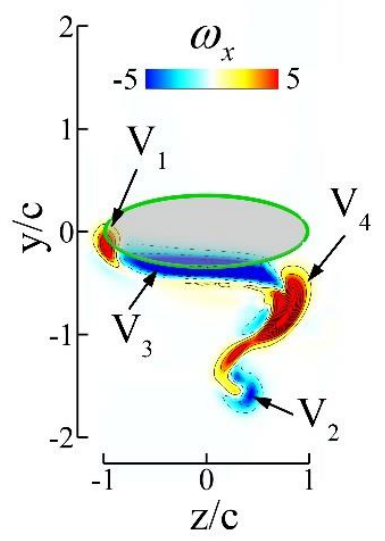

(f)

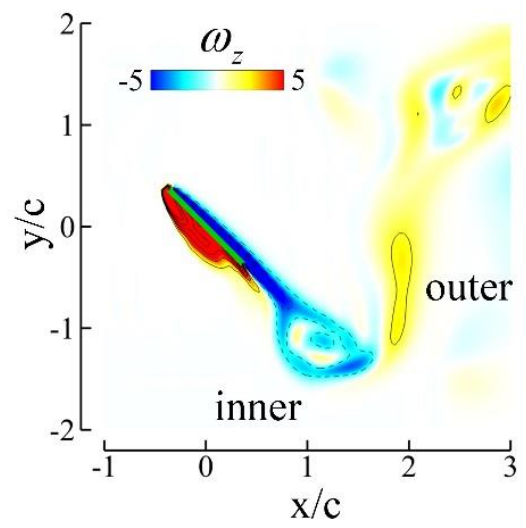

Figure 4-15. Comparison of $2-\mathrm{D}$ vorticity contours for $A R=1.27$ (a, d), $A R=1.91$ (b, e) and $A R=2.55$ (c, f). Figures (a-c) are the streamwise vorticity $\left(\omega_{x}\right)$ contours at the location near the plate trailing edge and (d-f) are the spanwise vorticity $\left(\omega_{z}\right)$ contours at the mid-span of the plates. The spatial locations of the slice cuts are shown as red planes in (a) and (d), respectively. 

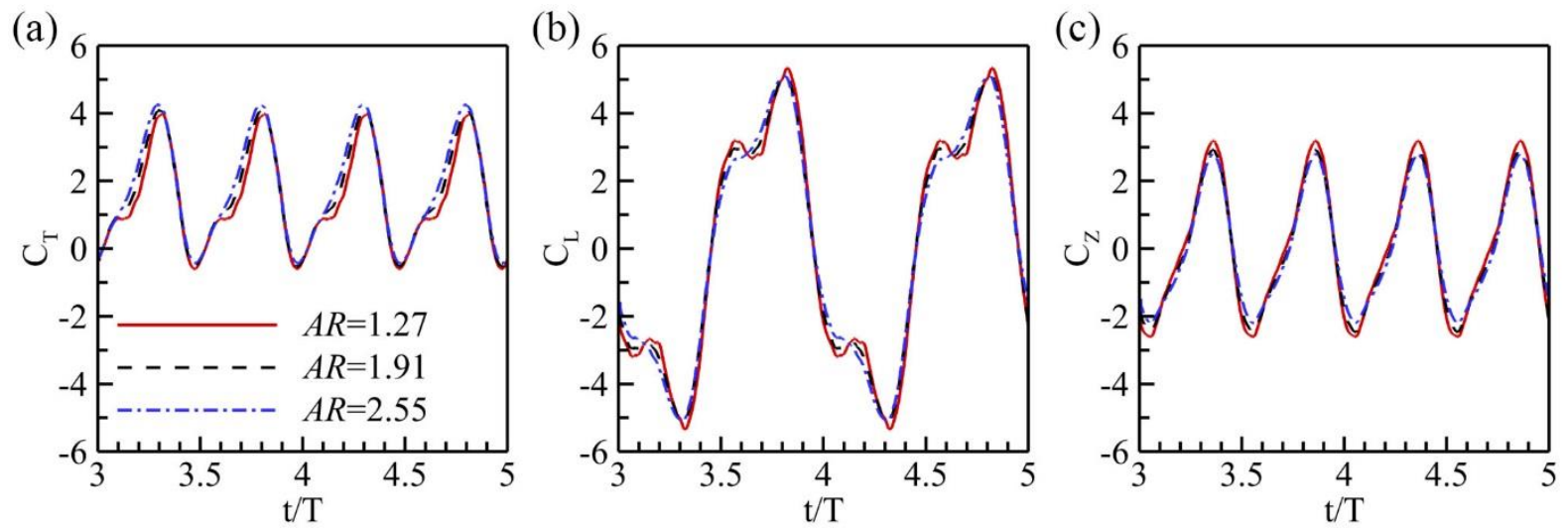

Figure 4-16. Computed time-varying force coefficients for the plate with various aspect ratios $(A R)$. (a) Thrust coefficient, (b) lift coefficient, and (c) spanwise force coefficient. The Strouhal number and Reynolds number are fixed at 0.6 and 200, respectively, for all these cases.

\subsubsection{Effects of Phase Difference between Pitching and Rolling}

In previous sections, we discussed the wake topology and force generation of pitchingrolling plates with phase difference angle $(\psi) 90^{\circ}$ between pitching and rolling motion at various Strouhal numbers, Reynolds numbers, and aspect-ratios. In the current section, we examine the effect of the phase difference angle on the vortical structures and hydrodynamic performance for an $A R=1.27$ circular plate at $S t=0.6$ and $R e=200$.

Figure 4-17 shows both side and top views of wake topology for $\psi=100^{\circ}, 110^{\circ}$ and $120^{\circ}$, respectively. This plot can be examined in conjunction with the same view for the $\psi=90^{\circ}$ cases in Figure 4-7 and Figure 4-10. Due to the self-induced vortex ring velocity, the wake topology of $\psi=90^{\circ}$ cases shown in previous sections (i.e. Figure 4-10 (b, d) and Figure 4-7 (c)) present slight wake deflection in the spanwise direction towards the plate tip. However, as the phase difference angle $(\psi)$ increases from $90^{\circ}$ to $120^{\circ}$, the wake gradually deflects from the tip 
direction to the root direction as illustrated in the right column of Figure 4-17. This wake deflection angle $(\gamma)$ can reach to $-7^{\circ}$ in $\psi=120^{\circ}$ case. Here, the positive and negative sign of $\gamma$ represents the wake deflection towards plate tip and plate root, respectively. Another salient feature of the wake topology is the wake oblique angle $(\alpha)$ incrementing along with the increasing of phase difference angle. The iso-surface of vorticity shows that slight changing on the phase difference between pitching and rolling motion can enhance the vorticity strength of shed vortex ring and thus led to the increasing of mutual induction between two adjacent vortex rings. The obtained wake oblique angles and wake deflection angles with respect to the phase difference angles are listed in Table 4-5.

Table 4-5. Effects of phase difference on the wake oblique angle $(\alpha)$ and wake deflection angle $(\gamma)$ at $S t=0.6$ and $R e=200$.

\begin{tabular}{ccc}
\hline \hline$\psi$ & $\alpha\left(^{\circ}\right)$ & $\gamma\left(^{\circ}\right)$ \\
\hline 90 & 21 & 5 \\
100 & 22 & -2 \\
110 & 25 & -5 \\
120 & 27 & -7 \\
\hline \hline
\end{tabular}

The enhancement of vorticity strength due to the increment of phase difference is also evident on the force generation. Figure 4-18 shows the instantaneous force coefficients for $\psi$ ranging between $100^{\circ}$ and $120^{\circ}$ together with the baseline case $\left(\psi=90^{\circ}\right)$. This plot shows that the peak values of all three force components are significantly increased when the phase 
difference angle is varied. It should be noted that the production of large force peaks can be extremely useful for performing rapid manoeuvers. Thus, the phase difference angle can be treated as a potential control parameter for bio-robotic design. In addition to the instantaneous force history, the cycle-averaged performance of different phase angles are evaluated and shown in Figure 4-19. The figure shows that a slight change in the phase difference angle can increase both the cycle-averaged thrust and efficiency compared to the baseline case $\left(\psi=90^{\circ}\right)$. In particular, the interpolation of these data indicates that the optimal propulsion efficiency can be obtained for a phase difference angle of approximately $105^{\circ}$ (pitching leads the rolling motion), which can lead to the enhancement of the cycle-averaged thrust and efficiency of up to $23 \%$ and $15 \%$, respectively. It is worth noting that current optimal phase difference angle ( around $105^{\circ}$ ) is different from that found in the pitching-heaving foils [113]. The previous numerical investigation of pitching-heaving foils found that the foils perform most efficiently when the phase shift angle was set up between $70^{\circ}$ and $90^{\circ}$. This different phase angle preference is because of the unique wake structures produced by pitching-rolling plates comparing to that formed by the pitching-heaving plate. As shown in Figure 4-17, the wake deflection angles $(\gamma)$ are affected by the change of the phase difference angle. When the phase difference angle is above $90^{\circ}$, the wake convects to the downstream with the minimum deflection. Figure 4-19 also shows that the plate reaches to the maximum efficiency between $100^{\circ}$ and $110^{\circ}$. 
(a)
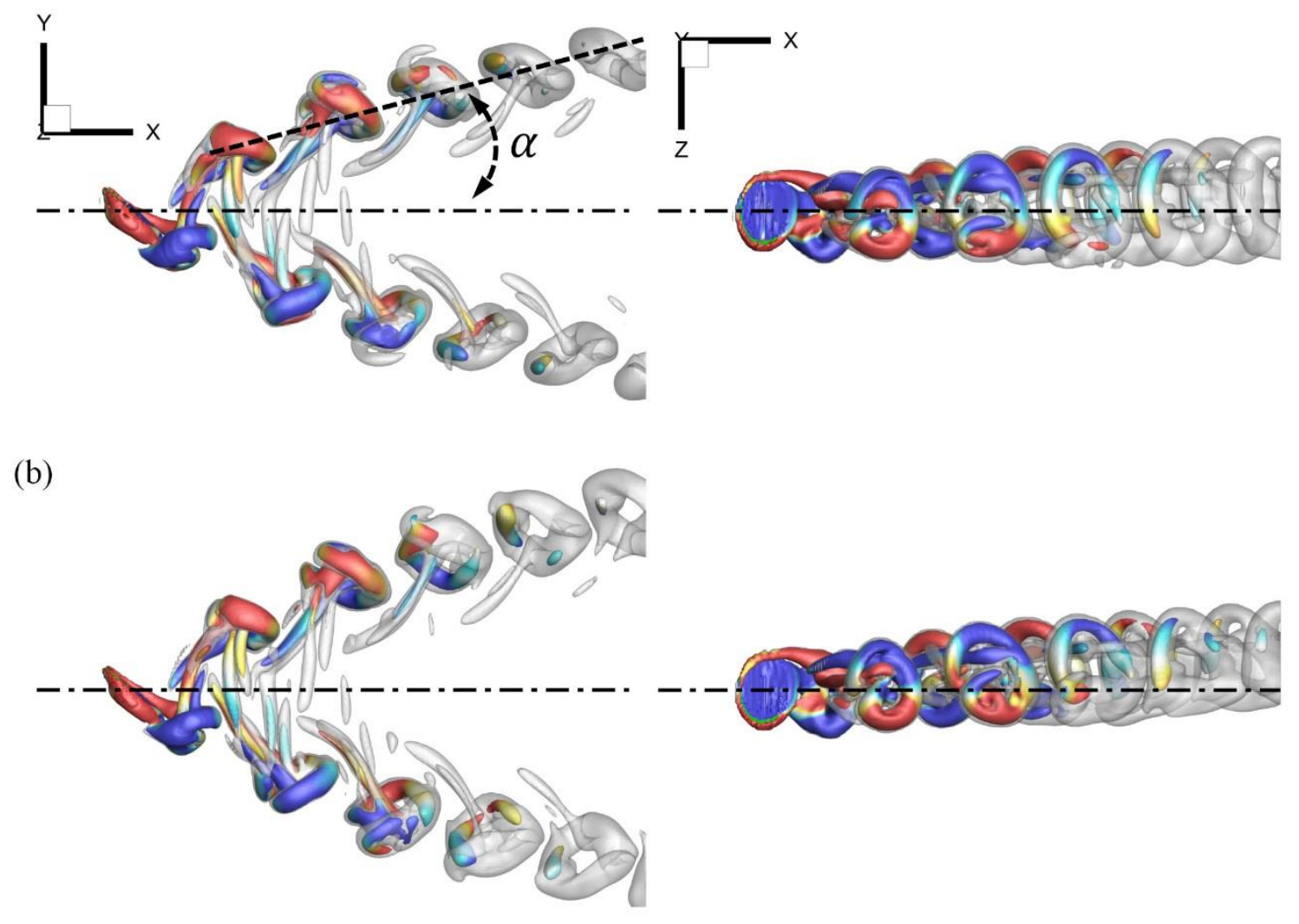

(c)

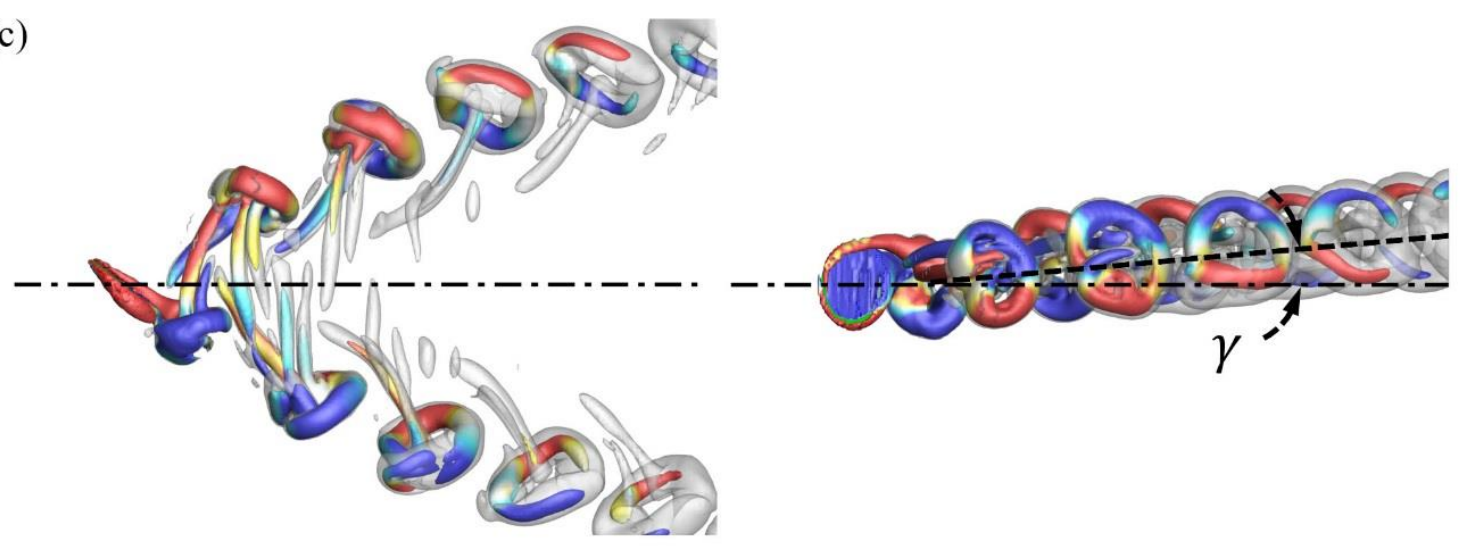

Figure 4-17. Effects of phase difference between pitching and rolling motions on the wake structure for the $A R=1.27$ circular plate. (a) $\psi=100^{\circ}$. (b) $\psi=110^{\circ}$. (c) $\psi=120^{\circ}$. The left and right columns show the side view and top view, respectively. The Strouhal number, Reynolds number and aspect-ratio are fixed at 0.6, 200 and 1.27, respectively, for all these cases. 

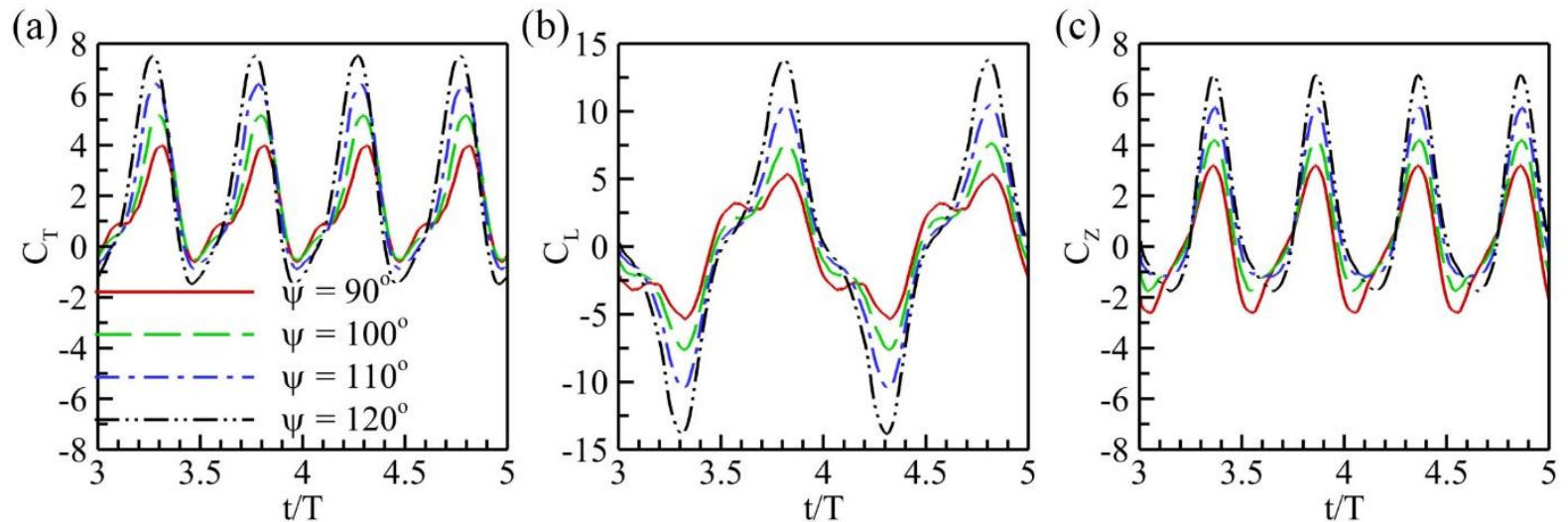

Figure 4-18. Computed time-varying force coefficients for the plate with various phase difference between pitching and rolling motion $(\psi)$. (a) Thrust coefficient, (b) lift coefficient, and (c) spanwise force coefficient. The Strouhal number and Reynolds number are fixed at 0.6 and 200, respectively, for all these cases.

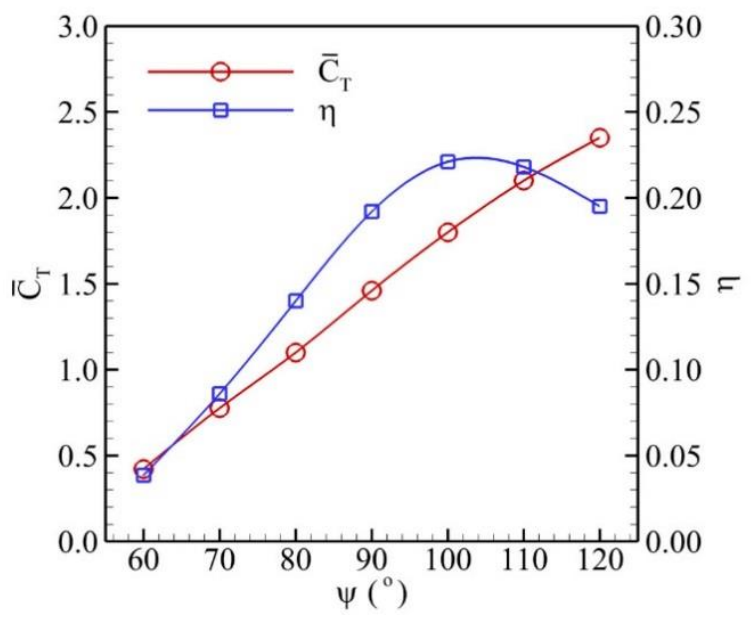

Figure 4-19. Variation in the cycle-averaged hydrodynamic performance for the baseline case $\left(\psi=90^{\circ}\right)$ as a function of phase difference between pitching and rolling motion. The red and blue curves indicate the mean thrust coefficient and propulsive efficiency, respectively. The Reynolds number and Strouhal number for all these cases are set to 200 and 0.6 , respectively. 


\subsection{POD Analysis of Wake Topology}

The wake topology of the baseline case is examined in this section using proper orthogonal decomposition (POD). The vortex structures are visualized by the Q-criterion [94], and colored by the streamwise vorticity $\left(\omega_{x}\right)$ for indicating the direction of vortices. Figure 4-20 shows a bifurcating wake pattern at two different phase $t / T=4.0$ and 4.5 . At the instant $t / T=4.0$, the plate is at the lowest position and starting to move upward. As the plate moving upward, the new formed vortices connect and form vortex loop. A complete vortex ring will form after the leading vortex is shed at $t / T=4.5$. Note that the rolling motion creates different shear rates between plate root and tip on the evolution of the vortex loop. This breaks the XY-plane time-invariant symmetry topology in pitching-heaving motion as mentioned in Ref. [27]. Only the spatiotemporal symmetry of the flow exist in the current study, which can be written as:

$$
R_{x z} \cdot(\mathrm{u}, \mathrm{v}, \mathrm{w})(\mathrm{x}, \mathrm{y}, \mathrm{z}, \mathrm{t})=(\mathrm{u},-\mathrm{v}, \mathrm{w})(\mathrm{x},-\mathrm{y}, \mathrm{z}, \mathrm{t}+0.5 \mathrm{~T})
$$

where, $R_{x z}$ is an action representing the spatio-temporal reflection about the XZ-plane, and $0.5 \mathrm{~T}$ is the half of a period.

Because the vorticity is a pseudo-vector, the signs of the velocity and vorticity components are opposite for the reflective symmetry. Meanwhile, the corresponding vorticity symmetry can be derived from Eq. (4-6):

$$
R_{x z}^{\prime} \cdot\left(\omega_{x}, \omega_{y}, \omega_{z}\right)(\mathrm{x}, \mathrm{y}, \mathrm{z}, \mathrm{t})=\left(-\omega_{x}, \omega_{y},-\omega_{z}\right)(\mathrm{x},-\mathrm{y}, \mathrm{z}, \mathrm{t}+0.5 \mathrm{~T})
$$

where, $R_{x z}$ is an action representing the spatio-temporal reflection about the XZ-plane, and $0.5 \mathrm{~T}$ is the half of a period. 
(a)

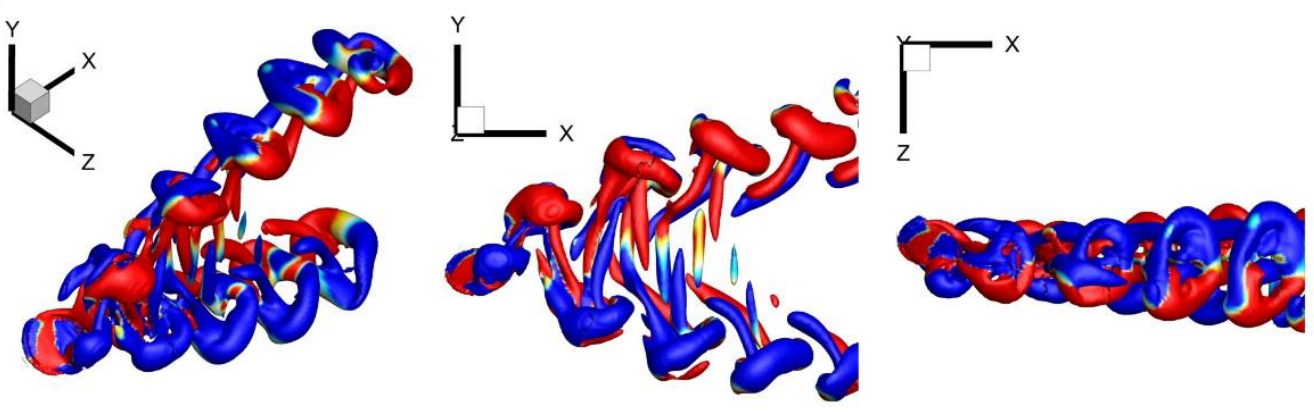

(b)
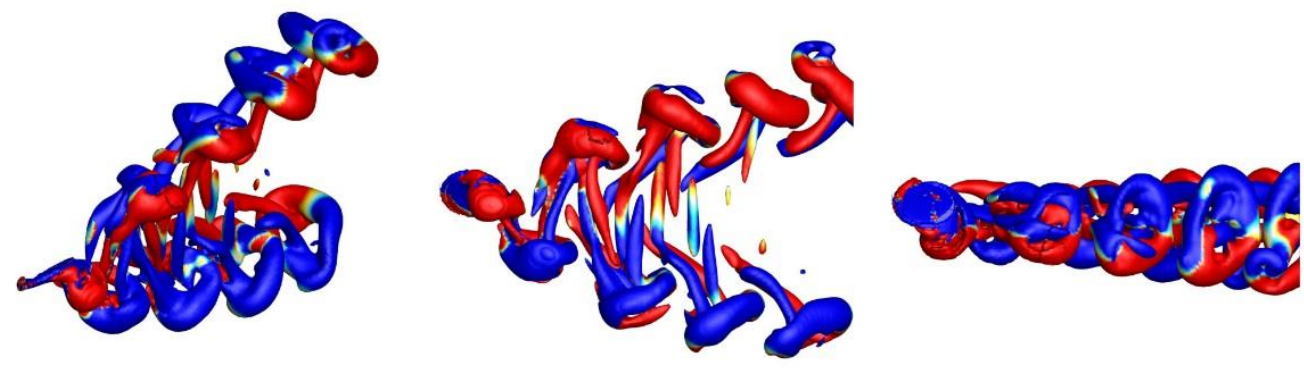

$-0.25$

0.25

Figure 4-20. Wake topology of circular plate $(\mathrm{AR}=1.27)$ with $\mathrm{St}=0.6$ and $\mathrm{Re}=200$ at two different phase. (a) The plate is at the lowest point in its rolling motion and starting to move upward (t/T=4.0). (b) The plate is at the highest point in its rolling motion and starting to move downward ( $\mathrm{t} / \mathrm{T}=4.5)$. To easily identify the vorticity transpose direction along $\mathrm{x}$-axis, the vortex ring $(\mathrm{Q}=0.2)$ is colored by the streamwise vorticity $\left(\omega_{x}\right)$.

To ensure the spatio-temporal symmetry of the wake structure, the flow in the $5^{\text {th }}$ pitchingrolling cycle is chosen for conducting symmetry and POD analysis in this study. The computation of the POD modes performed in the same domain as the original flow.

Figure 4-21 shows the mean flow wake by averaging the data ensemble. Its vortex structure contains four major tubes and two small tubes. These structures are spatially antisymmetric about 
the XZ-plane, as indicated by the averaged X-direction vorticity $\left(\bar{\omega}_{x}\right)$. This is different from the mean flow of pitching-heaving plate, in which the wake are spatial antisymmetric about both $\mathrm{XY}$ - and XZ-plane.
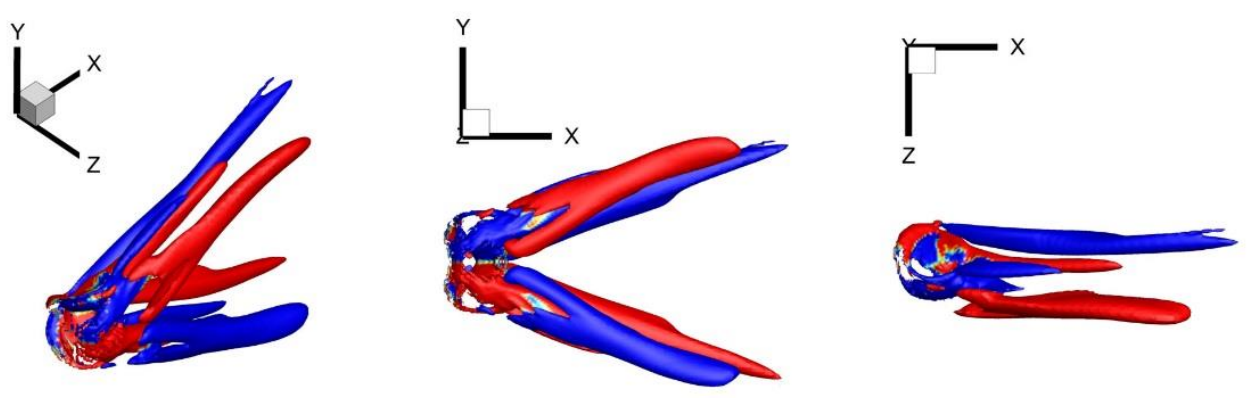

Figure 4-21. Iso-surfaces of Q-criterion of mean vorticity colored by the X-direction vorticity ( $\left.\bar{\omega}_{x}\right)$.

Contribution of individual eigenvalues to the total fluctuating kinetic energy with respect the mean flow wake can be expressed as normalized eigenvalues in a form of $\lambda_{i} / \sum_{k=1}^{N_{m}} \lambda_{k}$. Captured energy by the first $i$ modes can be represented by $\sum_{k=1}^{i} \lambda_{k} / \sum_{k=1}^{N_{m}} \lambda_{k}$. The values of the first eight modes are listed in Table 4-6. Curves of normalized eigenvalues and captured energy with respect to the number of POD modes are shown in Figure 4-22. It is worth noting that every two eigenvalues form a pair with approximate values for the first several modes. The first two modes contain about $38 \%$ and $35.9 \%$ of the total fluctuating kinetic energy, respectively. The third and fourth modes account for lower energy at about $7 \%$. The cumulative energy up to the $8^{\text {th }}$ mode is over $95 \%$. This means that the first eight modes capture a majority of large flow features 
including the vortices near the flapping wing and the vortices in the far wake zone. This is in consistent with previous studies on flapping wings [27,114].

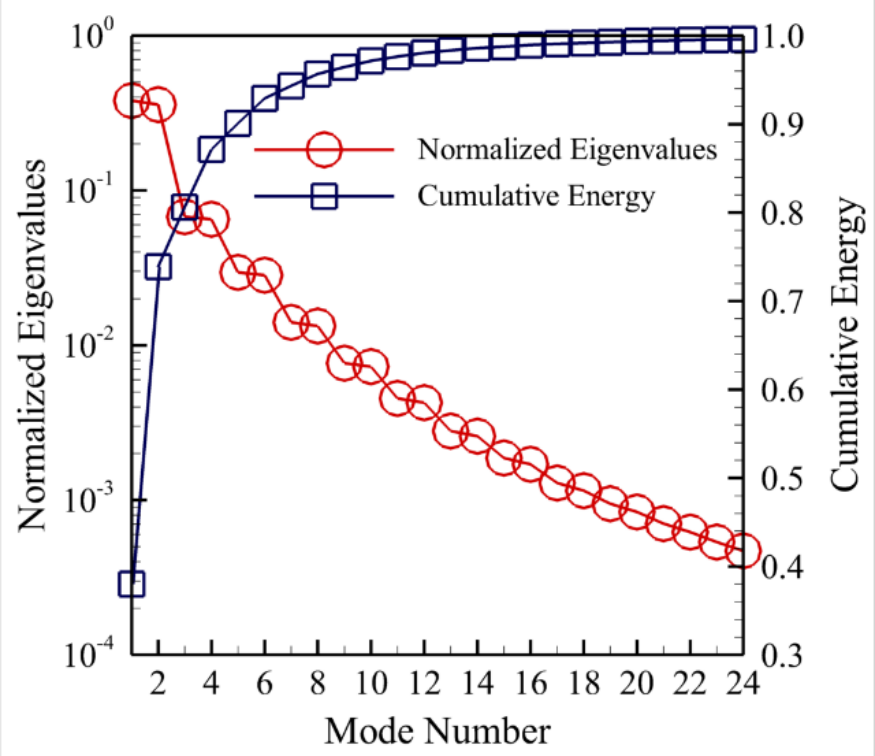

Figure 4-22. Normalized eigenvalues (red circle) and captured energy by the first $i$ modes (blue square) versus mode number $i=1,2, \ldots, 24$.

The wake topology of POD modes 1 to 8 are shown in Figure 4-23. The first two modes contain a chain vortices switch rotational direction between clockwise and counterclockwise when viewed from the top. The topology pattern of the mode 2 is one quarter wavelength shit of each vortex rings along the vortex chains. The topology structure of the modes 3 and 4 contain vortex tubes between the two sets of vortex chains. There is $\pi / 2$ phase difference between the modes 3 and 4 as indicated by the color switching of X-direction vorticity. The rest of modes pair have the similar topology structures as that of the modes 3 and 4 . Here the phase shifting is 
indicated by the color change of vorticity. It is also shown that the fluctuating kinetic energy dissipates rapidly in the downstream for the higher modes.

Table 4-6. Contribution of the first eight eigenvalues to the total fluctuating kinetic energy.

\begin{tabular}{ccc}
\hline \hline Mode & Contribution (\%) & Accumulation (\%) \\
\hline 1 & 38.0 & 38.0 \\
2 & 35.9 & 73.9 \\
3 & 6.7 & 80.6 \\
4 & 6.5 & 87.1 \\
5 & 3.0 & 90.1 \\
6 & 2.8 & 92.9 \\
7 & 1.4 & 94.3 \\
8 & 1.4 & 95.7 \\
\hline \hline
\end{tabular}

Similar as previous two-dimensional $[115,116]$ three-dimensional $[27,114]$ studies, the streamwise velocity of the POD modes 1 and 2 is antisymmetric about the horizontal line/plane. By contrast, the modes 3 and 4 are symmetric. The same behavior is also found in the current wake topology because of the existence of the spatio-temporal symmetry. The every-two-mode pattern is caused by the periodicity of the flow. This symmetry pattern are distinguishable in the topology of the POD modes, as listed in Table 4-7. The terms " $S$ " and " $A$ " denote symmetric and antisymmetric respect to the XZ-plane, respectively. According to symmetry, the POD modes can be categorized into two groups, $G_{1}=\{x$ : mean, modes $3,4,7,8, \ldots 19,20,23,24\}$ and 
$G_{-1}=\{x:$ modes $1,2,5,6, \ldots 17,18,21,22\}$. This classification holds for the pitching-heaving plate cases [27], as well. It is worth to point out that the symmetry property of the POD modes is independent of the selection of domain size for computing the POD modes.

Table 4-7. Vorticity symmetry of the mean flow and POD modes.

\begin{tabular}{|c|c|c|c|c|c|c|c|c|c|c|c|c|c|c|c|c|c|}
\hline & \multicolumn{3}{|c|}{ Mean flow } & \multicolumn{3}{|c|}{ Mode 1, 2} & \multicolumn{4}{|c|}{ Mode 3, 4} & \multicolumn{4}{|c|}{ Mode 5, 6} & \multicolumn{3}{|c|}{ Mode 7, 8} \\
\hline Vorticity & & $\bar{\omega}_{y}$ & & & y & $\psi_{z}$ & 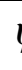 & & 'y & $\psi_{z}$ & & $\psi_{x}$ & $\psi_{y}$ & $\psi$ & $\psi_{x}$ & $\psi_{y}$ & $\psi_{z}$ \\
\hline XZ-plane & $A$ & $S$ & A & & 4 & $S$ & & & $S$ & $A$ & & $S$ & $A$ & $S$ & $A$ & $S$ & $A$ \\
\hline
\end{tabular}

The XZ-plane symmetry of the velocity components of the POD modes of $G_{1}$ and $G_{-1}$ can be written as Eq. (4-7) and (4-8), respectively.

$$
\begin{gathered}
r_{x z} \cdot\left(\Phi_{x}, \Phi_{y}, \Phi_{z}\right)(\mathrm{x}, \mathrm{y}, \mathrm{z})=\left(\Phi_{x},-\Phi_{y}, \Phi_{z}\right)(\mathrm{x},-\mathrm{y}, \mathrm{z}) \\
-r_{x z} \cdot\left(\Phi_{x}, \Phi_{y}, \Phi_{z}\right)(\mathrm{x}, \mathrm{y}, \mathrm{z})=\left(-\Phi_{x}, \Phi_{y},-\Phi_{z}\right)(\mathrm{x},-\mathrm{y}, \mathrm{z})
\end{gathered}
$$

Where, $r_{x z}$ is the spatial reflection about the XZ-plane, and the velocity components $\Phi_{x}, \Phi_{y}$ and $\Phi_{z}$ are independent on $t$. 

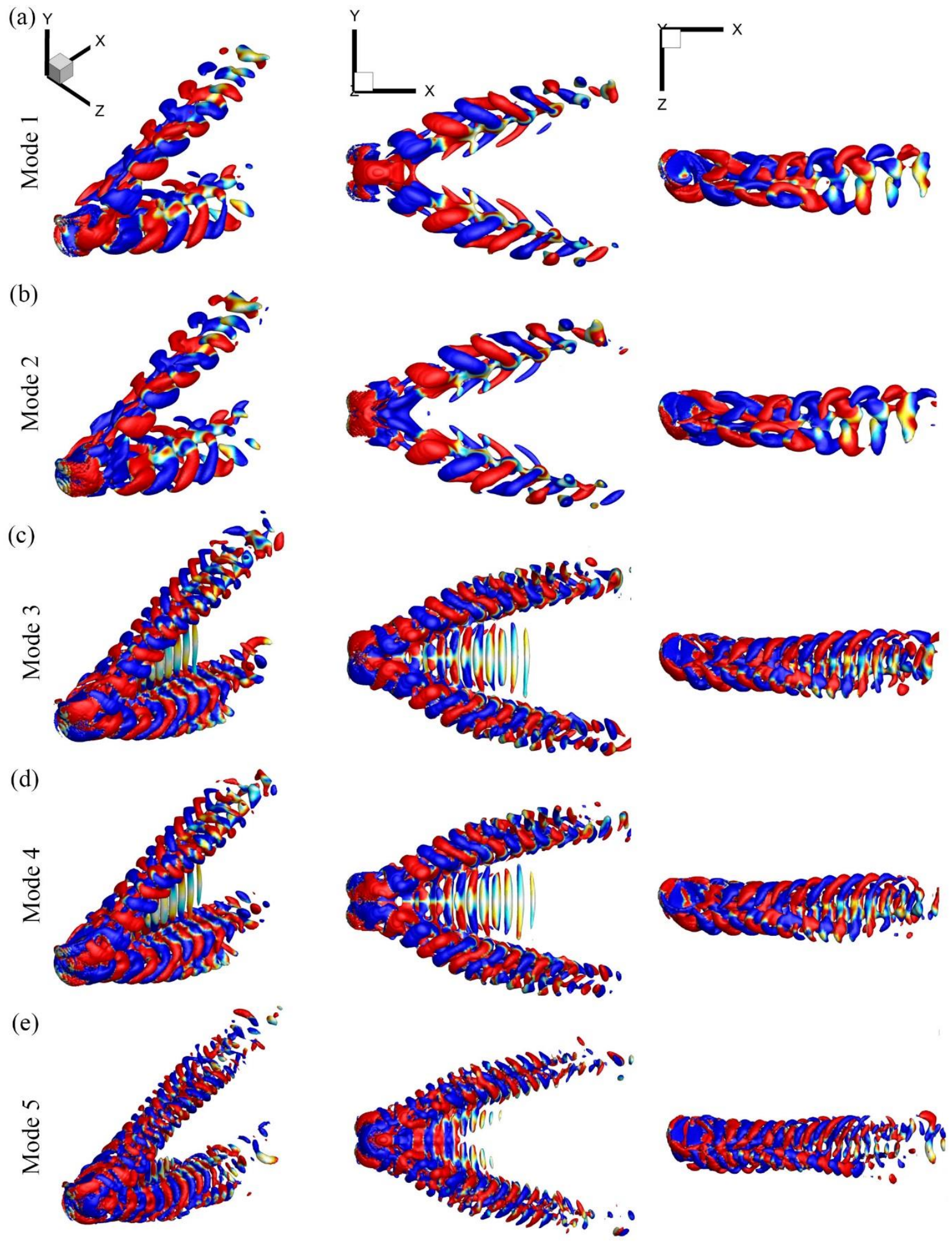
(f)
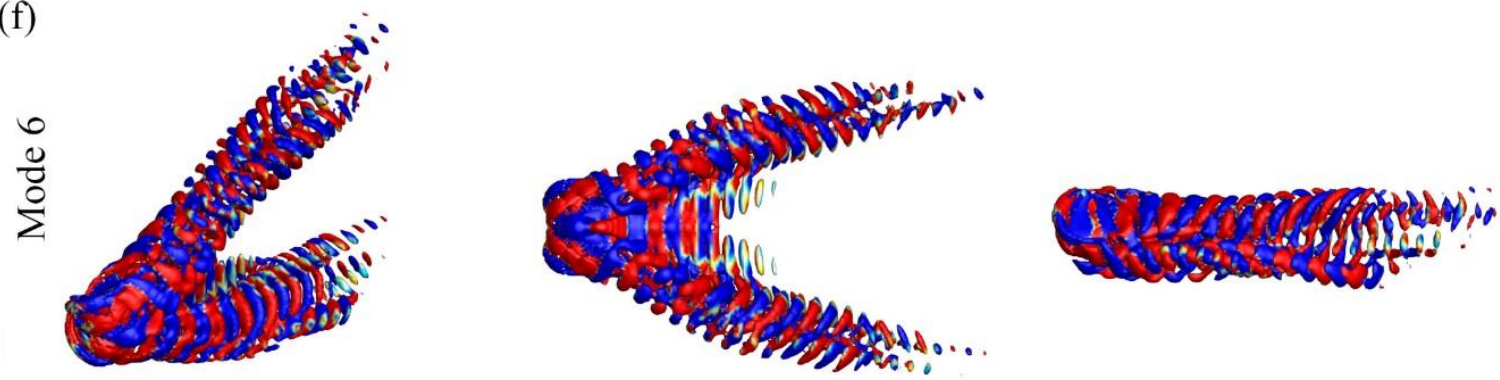

(g)
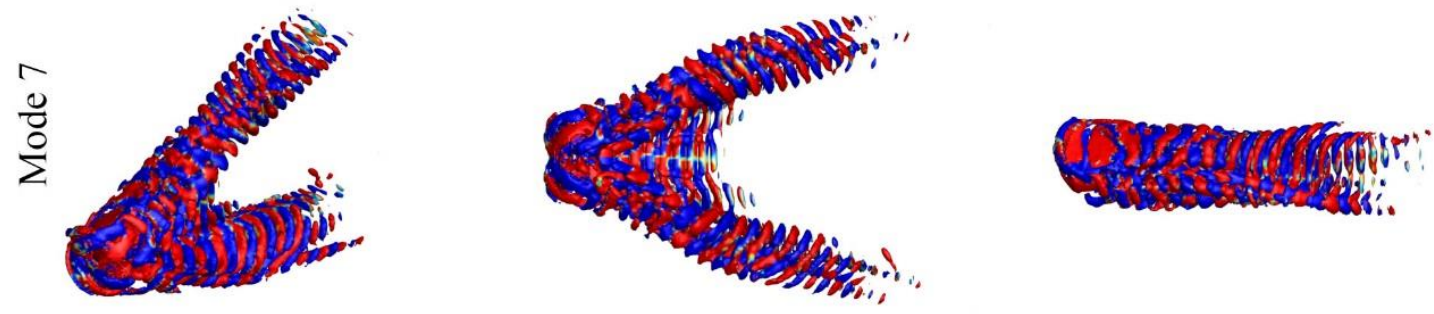

(g)
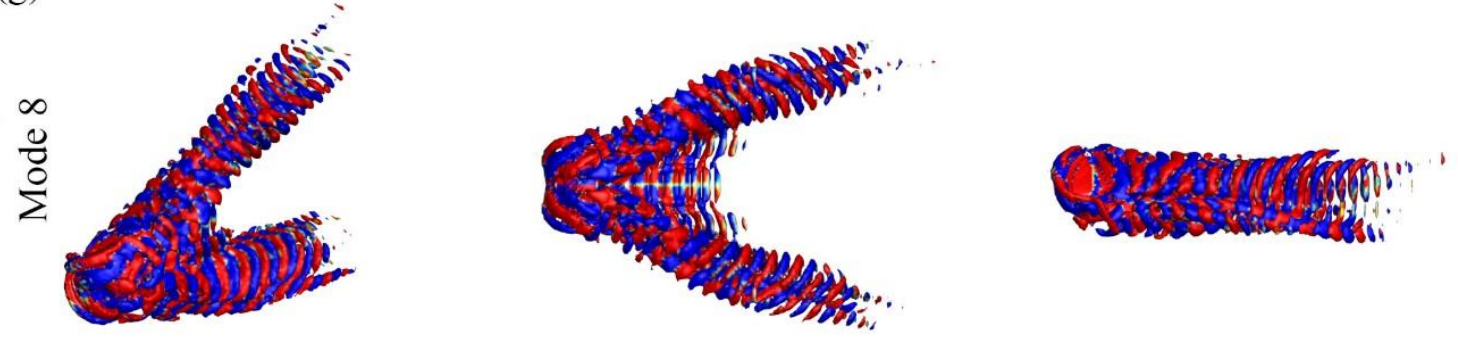

Figure 4-23. Iso-surface of Q-criterion of POD modes from 1 to 8 . The iso-surface of the modes are colored by the X-direction vorticity.

Thus, the relative vorticity symmetry can be calculated based on Eq. (4-9) and (4-10):

$$
\begin{aligned}
& r_{x z}^{\prime} \cdot\left(\psi_{x}, \psi_{y}, \psi_{z}\right)(\mathrm{x}, \mathrm{y}, \mathrm{z})=\left(-\psi_{x}, \psi_{y},-\psi_{z}\right)(\mathrm{x},-\mathrm{y}, \mathrm{z}) \\
& -r_{x z}^{\prime} \cdot\left(\psi_{x}, \psi_{y}, \psi_{z}\right)(\mathrm{x}, \mathrm{y}, \mathrm{z})=\left(\psi_{x},-\psi_{y}, \psi_{z}\right)(\mathrm{x},-\mathrm{y}, \mathrm{z})
\end{aligned}
$$


The history of the temporal coefficients and the phase portraits respect to $\alpha_{1}$ are shown in Figure 4-24. The pairs $\left(\alpha_{1}, \alpha_{2}\right),\left(\alpha_{3}, \alpha_{4}\right),\left(\alpha_{5}, \alpha_{6}\right)$ and $\left(\alpha_{7}, \alpha_{8}\right)$ have a frequency of $f, 2 f, 3 f$ and $4 f$, respectively. For each pair, the phase difference is $\pi / 2$. The pattern sets $G_{1}$ and $G_{-1}$ have different temporal symmetries. By defining $\tau$ as the symmetry for the temporal coefficient, the temporal reflection of $G_{1}$ and $G_{-1}$ can be written as Eq. (4-11) and (4-12), respectively.

$$
\begin{gathered}
\tau \cdot \alpha_{i}(t)=\alpha_{i}(t+0.5 T) \\
(-\tau) \cdot \alpha_{i}(t)=-\alpha_{i}(t+0.5 T)
\end{gathered}
$$

Figure 4-25 shows the temporal variation in the force coefficients over two cycles when the forces reached a periodic stage and their power spectrum. In Figure 4-25 (a), the thrust peaks occurred twice during each cycle at the instant when the plate was near the center of its trajectory. A slight drag was produced when the plate started to reverse its rolling direction. The maximum and mean thrust coefficients for this case were found to be 3.98 and 1.46, respectively. Figure 4-25 (b) and (c) show the other two force components produced by the plate. Both forces have equivalent positive and negative variations over a cycle. The calculated mean lift and spanwise forces are 0.0 and 0.1 , respectively. From Figure 4-25(d-f), it is evident that the horizontal force components $\left(C_{T}\right.$ and $\left.C_{Z}\right)$ are attributed to the even harmonics of the POD modes, whereas the vortical force component $\left(C_{L}\right)$ is attributed to the odd harmonic. 

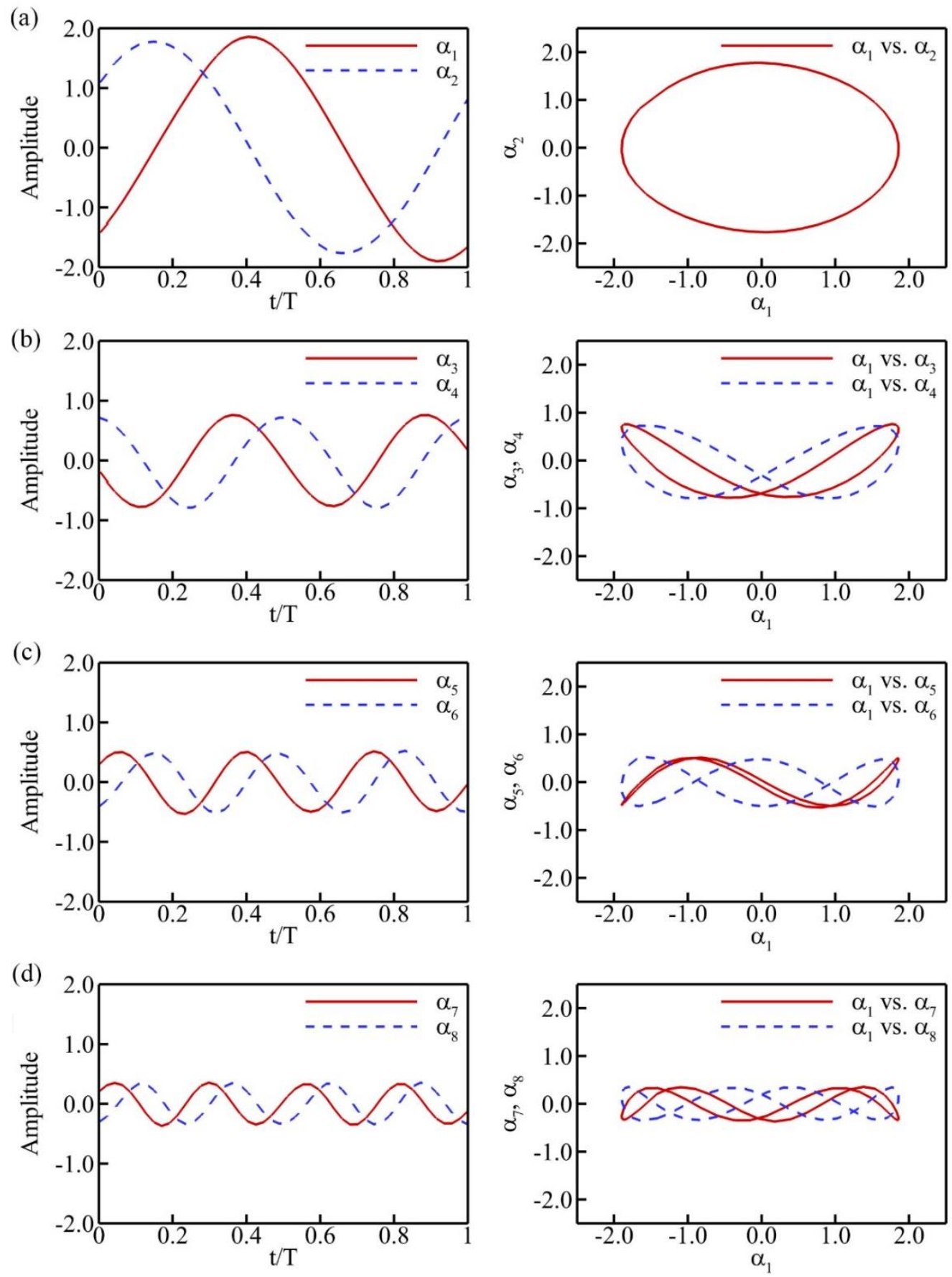

Figure 4-24. The history of the temporal coefficient and phase portraits of the coefficients respect to $\alpha_{1}$. 
(a)
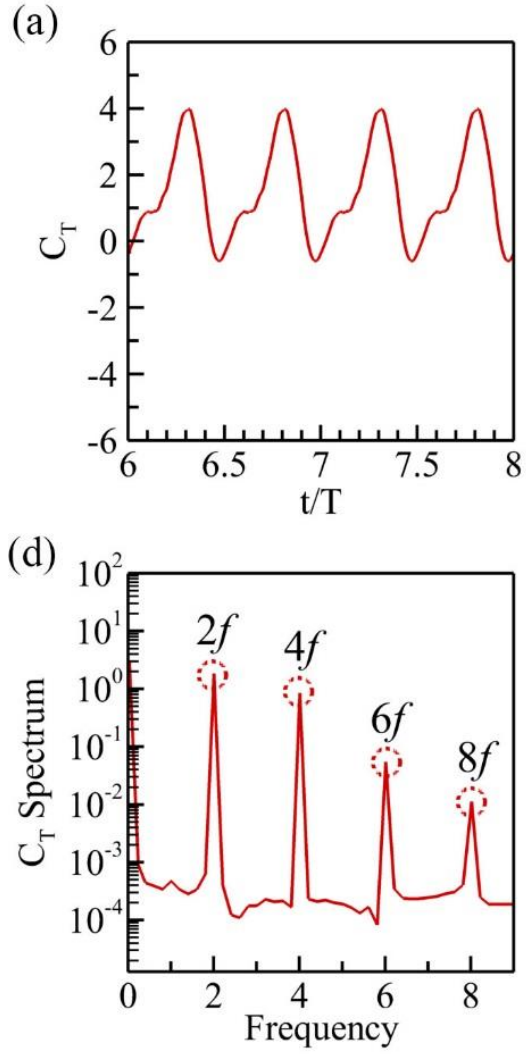

(b)

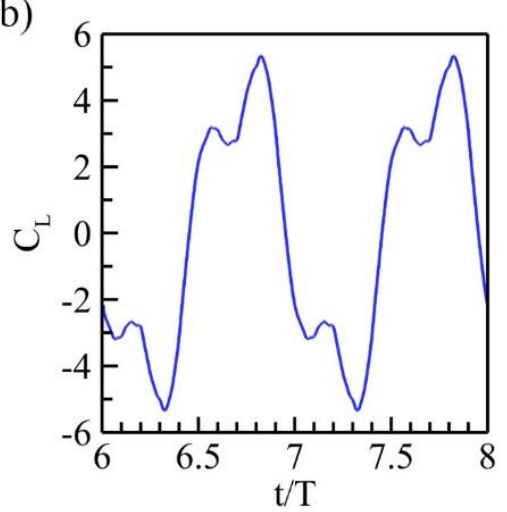

(e)

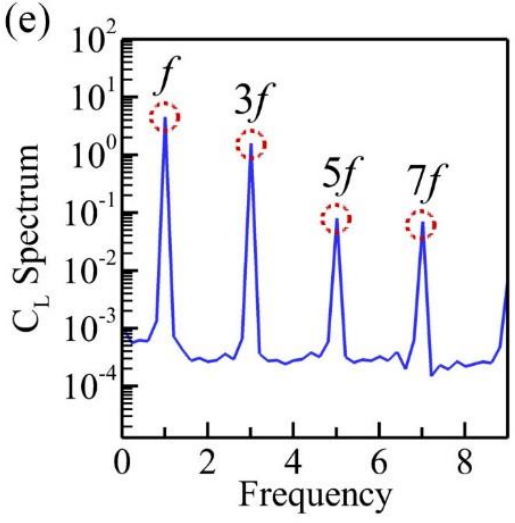

(c)

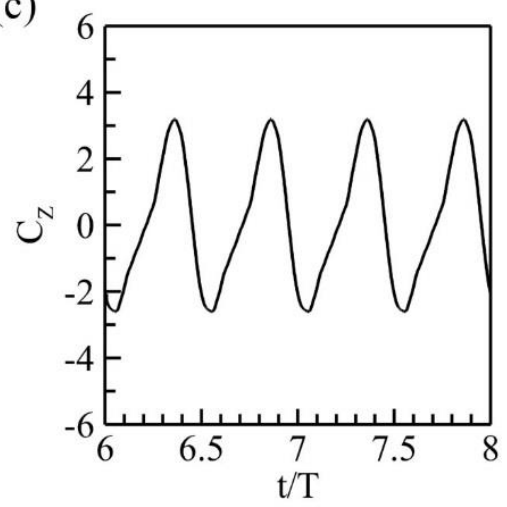

(f)

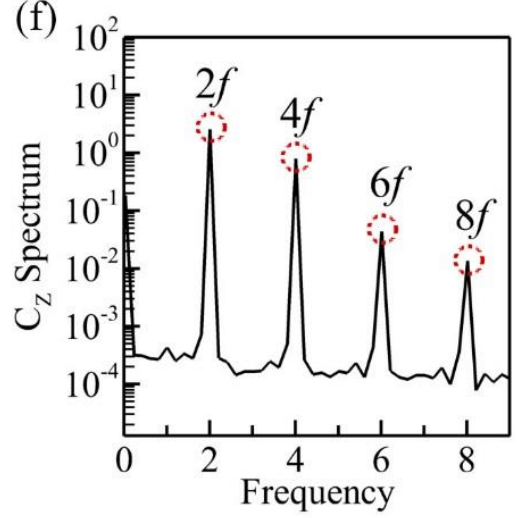

Figure 4-25. Thrust, lift, and spanwise force coefficients (a-c) from simulation results and its corresponding power spectrum (d-f).

Figure 4-26 shows comparison between the direct numerical simulation (DNS) results and the virtual force production calculated by using POD-FSM. The results have shown that the POD modes in $G_{1}$ only generate the horizontal force components and $G_{-1}$ only generate vertical force component. This is due to the same harmonics between the mode coefficients (Figure 4-24) and force coefficients (Figure 4-25). Once we group the POD modes by the odd and even harmonics, respectively, the virtual force production will only contribute to either vertical or horizontal forces. To illustrate the wake pattern of each group, Figure 4-27 shows the flow reconstructions 
by adding together the members of $G_{1}$ and $G_{-1}$, respectively. The reconstructions of both groups are in a form of chained vortex rings but with different vorticity directions.

(a)

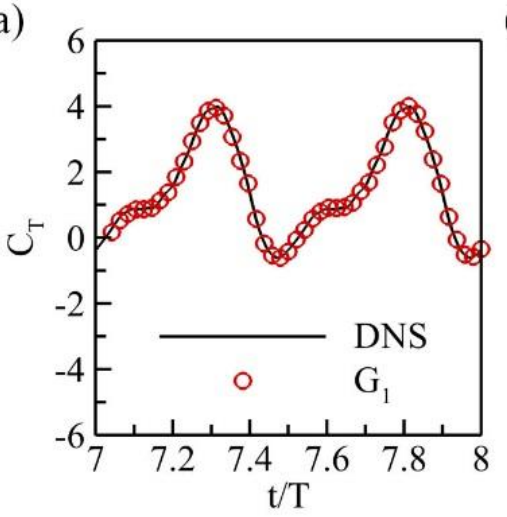

(d)

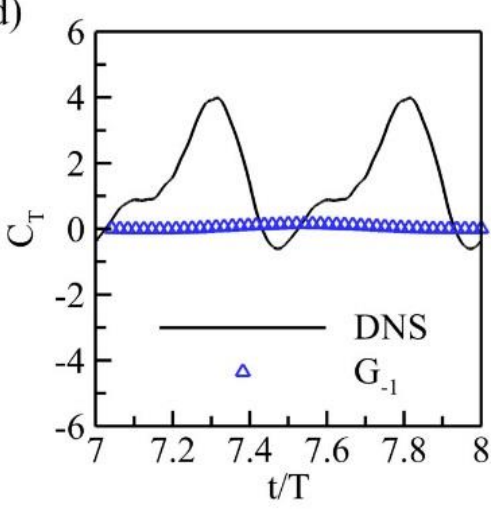

(b)

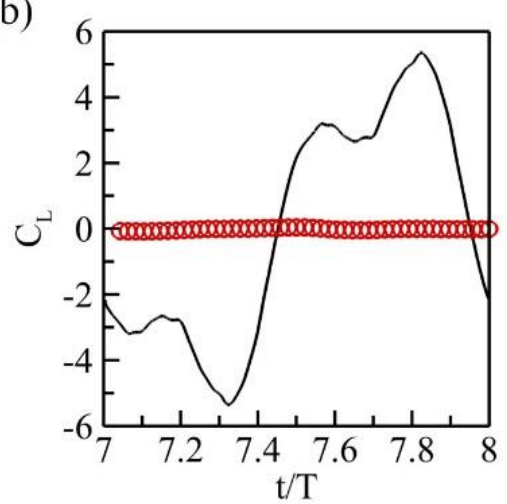

(e)

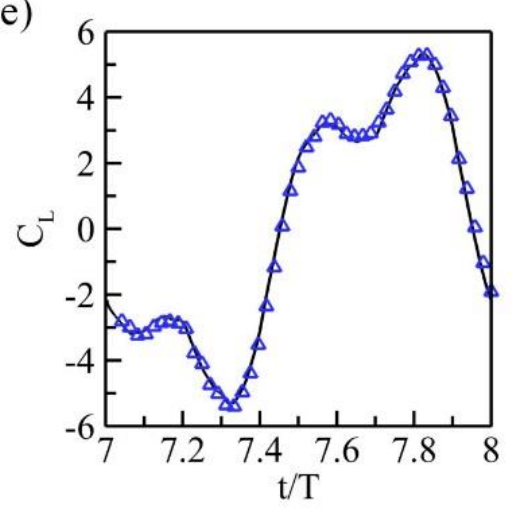

(c)

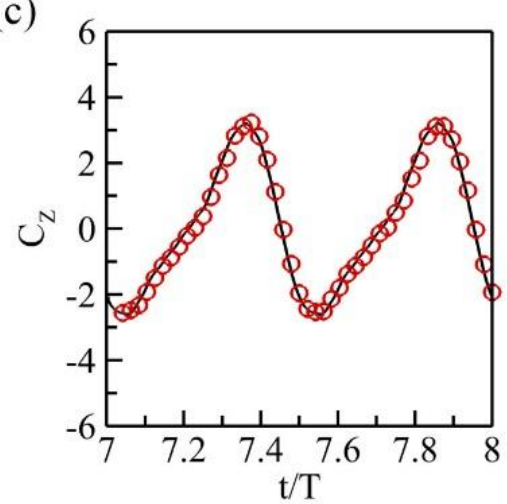

(f)

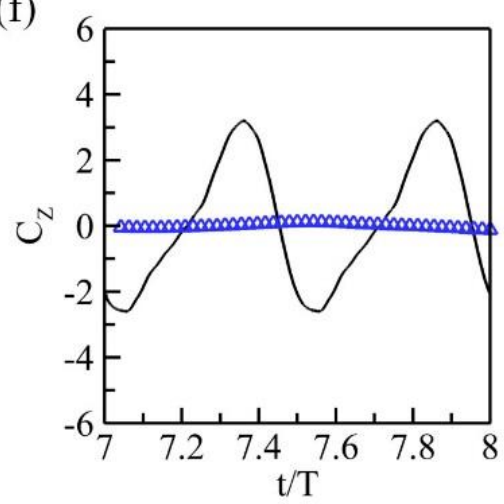

Figure 4-26. Thrust, lift, and spanwise force coefficients of $G_{1}(\mathrm{a}-\mathrm{c})$ and $G_{-1}(\mathrm{~d}-\mathrm{f})$ comparing with the DNS results (black line). 
(a)
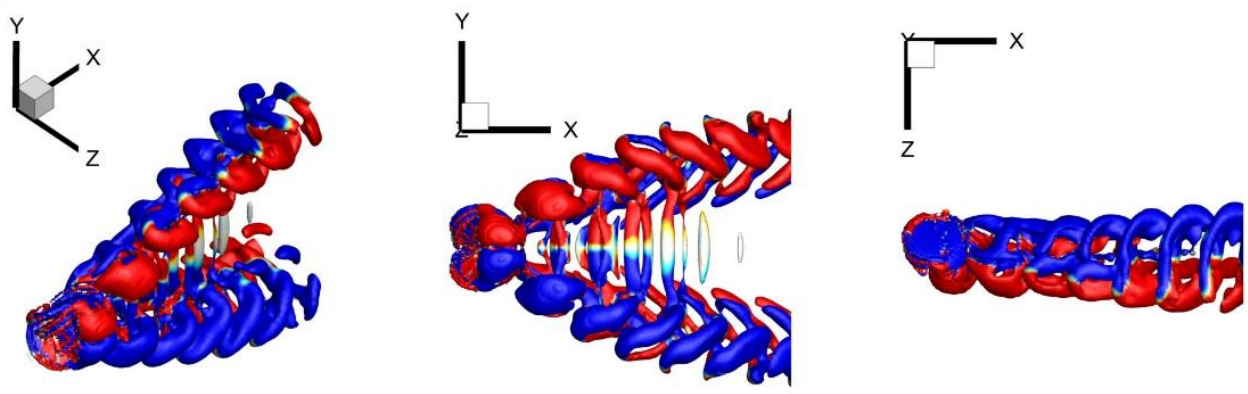

(b)
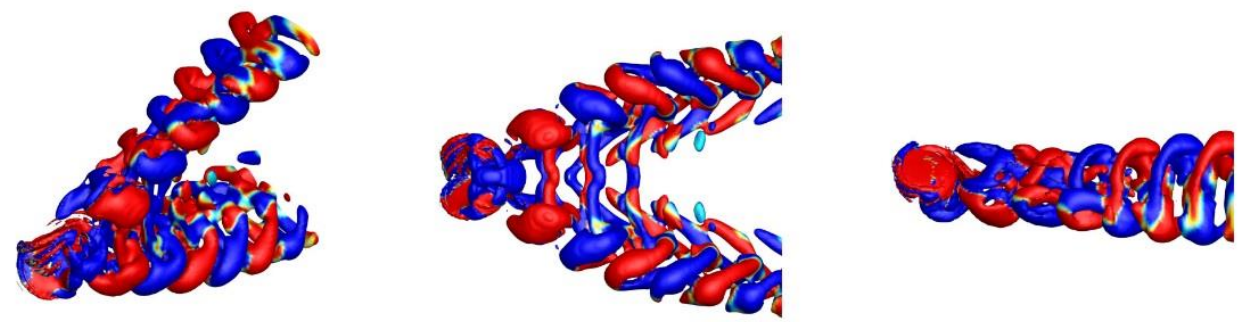

$-0.25$

0.25

Figure 4-27. Reconstructed flow fields of $G_{1}(\mathrm{a})$ and $G_{-1}(\mathrm{~b})$ at $\mathrm{t} / \mathrm{T}=7.0, \mathrm{Q}=0.2$.

\subsection{Chapter Summaries}

Simulations of flow past thin pitching-rolling plates have been conducted using an immersed boundary solver. Despite the simplified kinematics adopted to represent natural flapping propulsors, the current study is expected to provide some general insights into the aero/hydro-dynamics of low-aspect-ratio propulsors.

The simulations have shown that, in the near wake close to the plate trailing edge, a distinct double-loop vortical structure with opposite vortex sense can be observed. As the vortex loops shed into the wake, these two loops become connected with each other and form an interconnected "double-C"-shaped ring during each half flapping cycle. The periodically shed vortex rings eventually formed a bifurcating wake structure with a slight deflection in the 
spanwise direction to the plate tip. In addition, the contained vortex ring size gradually increases as it convects downstream. Persistence of the aforementioned wake formation is shown for a range of Strouhal numbers $(0.4 \sim 1.2)$, Reynolds numbers $(100 \sim 400)$, and plate aspect ratios ( $1.27 \sim 2.55$ ). Further analysis has shown that the formation and shedding process of this unique vortical structure resulted in double peaks in the force history. In addition, the cycle-averaged thrust was also found to increase monotonically with the Strouhal number, and the maximum propulsive efficiency can be achieved at a Strouhal number of approximately 0.6. Changing both the Reynolds number and plate aspect ratio affected the peak values of the force production but had little influence on the mean force magnitude. Our further investigation on the effects of phase difference angle between pitching and rolling motion indicated that the propulsive performance can be further improved if an appropriate phase difference angle is selected.

By conducting a POD analysis of the wake topology produced by a low-aspect-ratio pitching-rolling plate, we found that the first eight modes contain more than $95 \%$ fluctuating kinetic energy of the flow. All the POD modes show the spatio-temporal patterns about the central horizontal plane, however, the time-invariant symmetry topology observed in pitchingheaving motion [27] breaks down. Our results also indicated that the temporal coefficient of POD modes consist of odd and even harmonics, respectively, and repeat every two POD modes. By using a POD-FSM, it is found that the group of odd harmonic POD modes are only contribute to the vertical force generation, whereas the even harmonic POD modes are only contribute to the horizontal forces generation. This observation imply that the inherent connection between the POD modes and virtual force generations is independent of the time-invariant spatial symmetry property. 


\section{Aerodynamic Effects of Morphing Wings in Dragonfly Forward}

\section{Flight}

Although some encouraging progress have been made in advance our knowledge on flapping morphing wings $[90,117,118]$, most of the previous studies are more focused on the aerodynamic performance rather than the vortical structures. So far, there is still a lack of qualitative and quantitative descriptions on the vortex formation of deformable wings, which can lead to improved models for the design of biomimetic propulsors, and also provide a better understand of vorticity transport mechanisms of morphing wings in nature. The present effort is meant to fill some of the knowledge gaps in this regard. The high-speed photogrammetry system, 3-D surface reconstruction technology and numerical simulations are used to reveal the effects of morphing wings of a forward flight dragonfly. Specifically, the flapping morphing wing kinematics of a free-flight dragonfly are measured and quantified first. We then use the reconstructed model to explore the effects of morphing wings, first by removing camber while keeping the same time-varying twist distribution, and second by removing both the camber and the spanwise twist. Numerical simulations are carried out using an in-house immersed-boundarymethod-based direct numerical simulation solver. To get a better understand of the aerodynamic roles of morphing wings, the leading-edge vortex, the wing surface pressure distribution, and wake structures were analyzed and compared in detail for the model wings. Due to the similarity of morphing wings effects on the aerodynamics for both forewings and hindwings, only hindwings' results are presented in the current Chapter. 


\subsection{Data Acquisitions}

Dragonflies (Erythemis Simplicicollis) are selected for this study. The flight and wing kinematics of dragonflies were quantified using a high-speed photogrammetry setup composed of three synchronized Photron Fastcam SA3 60K high-speed cameras paired with Nikon Nikkor 50mmf/1.4D AF lenses. The cameras were calibrated for 3-D reconstruction using the direct linear transformation (DLT) method [119]. Given the wing flapping frequency of approximately $20-40 \mathrm{~Hz}$, the cameras were operated at $1000 \mathrm{~Hz}$ record rate with a shutter speed of 2 us and $1024 \times 1024$ pixel resolution.

We collected approximately 90 separated recording of dragonflies' free flying using the images from the calibrated high-speed video apparatus. These videos were then examined carefully, and one particular recording was selected for that the dragonfly was performing forward flight in a almost constant forward speed and for which the image acquisition from all three cameras was of high quality. Taking advantage of marker points we put on the wings before the video shooting and other easily identifiable natural markings, we reconstructed the 3D deforming wing kinematics at 108 instants during three flapping cycles by measuring the 3-D location of 16 total points on each wing surface from this recording. A 3-D model of each wing is then iteratively mapped onto the marker data, thereby providing a smooth parametric surface representation of the wing in its deformable shape. After that, the wing surfaces were smoothed via linear interpolation by holding the coordinates of the measured points. Once the smoothed mesh was obtained for complete flapping cycle, we used Furious interpolation by taking the first 20 terms to interpolate the surface mesh in time to produce a high temporal frequency (1000 time-steps per flapping cycle) input for our CFD solver. The measurement of dragonfly 
morphology is shown in Table 5-1. Further details regarding the current reconstruction method as well as the evaluation of reconstruction accuracy can be found in Ref.[87].

Table 5-1. Morphological data for the dragonfly in the current study.

\begin{tabular}{lc}
\hline \multicolumn{1}{c}{ Variable } & Value \\
\hline body mass $(\mathrm{mg})$ & 2560.5 \\
body length $(\mathrm{mm})$ & 40.70 .05 \\
flapping frequency $(\mathrm{Hz})$ & $28 \pm 1$ \\
fore-/hind-wing span length $(\mathrm{mm})$ & $33.1 / 32.90 .05$ \\
fore-/hind-wing mean chord length $(\mathrm{mm})$ & $7.5 / 8.60 .05$ \\
fore-/hind-wing area $\left(\mathrm{mm}^{2}\right)$ & $213.9 / 257.00 .05$ \\
fore-/hind-wing aspect-ratio & $5.1 / 4.20 .02$ \\
wing load $\left(\mathrm{N} / \mathrm{m}^{2}\right)$ & 5.330 .02 \\
\hline \hline
\end{tabular}

\subsection{Computational Setup}

As shown in Figure 5-1, the simulations have been carried out on a non-uniform Cartesian grid of size $231 \times 175 \times 201$ (about 8.1 million). The overall computation domain has a size of $30 \bar{c} \times 30 \bar{c} \times 30 \bar{c}$ in terms of the averaged wing chord length, and a cuboidal area around the dragonfly of size $5.0 \bar{c} \times 3.2 \bar{c} \times 4.0 \bar{c}$ has a high-resolution uniform grid $(\Delta \cong 0.019)$ which is designed to resolve the near-wake vortex structures. The stretching grids are applied in all three directions from the fine region to the outside boundaries. At the left-hand boundary, we provide a constant inflow velocity boundary condition. The right-hand boundary is the outflow boundary, 
which is provided with a zero streamwise gradient boundary condition for the velocity, allowing the vortices to convect out of this boundary without significant reflections. The zero-stress boundary condition is provided at all lateral boundaries. A homogeneous Neumann boundary condition is used for the pressure at all boundaries. The time step in this study is 0.001 to ensure stable solutions obtained throughout the simulation.

Grid independence is assessed by simulating the same case on two other grids: one with $50 \%$ more and the other with 50\% fewer grid points than the baseline grid. Both of these simulations produce a maximum $1 \%$ difference from the baseline in vertical force. The corresponding differences for the root-mean-square value of horizontal force are $1.3 \%$ and $0.88 \%$ respectively. These results indicate a high level of accuracy in the aerodynamic forces computed on the baseline grid, as well as the flow features that produce these forces.

The Reynolds number is defined by $\operatorname{Re}=U_{\infty} \bar{c} / v$, where the kinematic viscosity $v$ is around $1.56 \times 10^{-5} \mathrm{~m}^{2} \mathrm{~s}^{-1}$ for air at room temperature $\left(27^{\circ} \mathrm{C}\right)$ and $\bar{c}$ is the mean chord length $(7.5 \mathrm{~mm})$ of the forewing. The forward velocity $\left(U_{\infty}\right)$ was measured as $2.07 \mathrm{~m} \mathrm{~s}^{-1}$. Based on the measured data in this study, the Reynolds number is about 995 . The reduced frequency is defined as $k=f l / U_{\infty}$, where $f(28.6 \mathrm{~Hz})$ is the flapping frequency, and $l(32.1 \mathrm{~mm})$ is the length of the forewing from the wing root to the wing tip. Based on the measured data in this study, the reduced frequency is 0.44 . The advance ratio, defined as $J=U_{\infty} /(2 \Phi f l)$, is 0.78 , where $\Phi$ is the stroke amplitude of the forewing, with the value about $83^{\circ}$. The above parameters are all within the range of previous records for free-flight dragonflies [120,121].

For evaluating the aerodynamic performance, the instantaneous aerodynamic forces acting on the wing surface can be calculated from the pressure and stresses along its surface based on 
the solutions to the N-S equations $[26,122]$. The instantaneous aerodynamic power is the power needed to overcome air resistance and is defined as the surface integration of the inner product between the pressure and the velocity in each discretized element.

$$
p_{\text {aero }}=-\iint p \vec{n} \cdot \vec{u}_{c} d s
$$

Where, $\vec{n}$ and $d s$ are the unit normal direction and the area of each surface element, respectively.

(a)

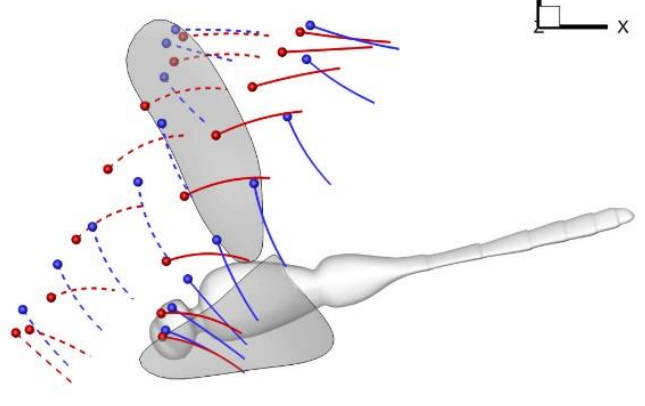

(b)

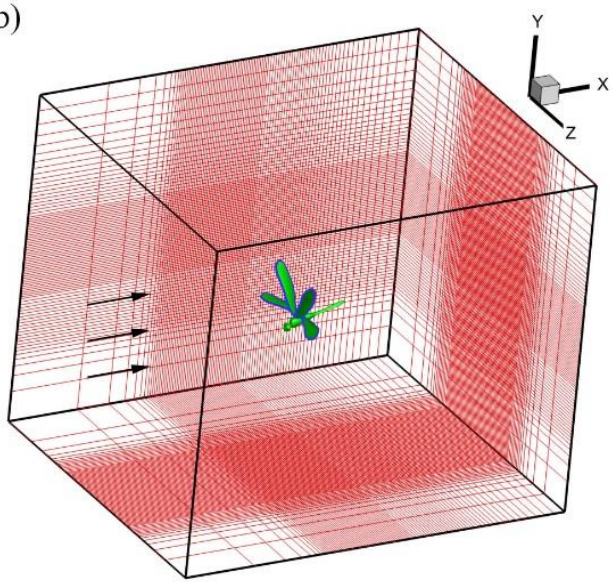

Figure 5-1. Flexible wing kinematics and computational setup. (a) Chord-lines of dragonfly forewing (dashed line) and hindwing (solid line) at a few instances. (b) Grid and domain employed for the cruising dragonfly simulations.

\subsection{Wing Kinematics and Surface Deformation}

To describe the motion of the wing about the body, we determine the stroke plane and wing kinematics in the same way as that of Ref.[99]. The stroke plane is determined by taking the averaged wing tip trajectory in the body frame, and its position about the body does not vary with time. The stroke plan angle is $73^{\circ}$ for the forewings and $77^{\circ}$ for the hindwings with respect 
to the horizontal plane. We then quantify wing kinematics based on the stroke plane by using three Euler angles: wing stroke $(\psi)$, wing deviation $(\phi)$, and wing pitch $(\theta)$. The definitions of wing kinematics parameters are shown in Figure 5-2, which describe the orientation of the wings relative to the wing root. Due to the wing flexibility, the wing pitching angle $(\theta)$ and the chordwise camber $(\mathrm{h} / \mathrm{c})$ are different along the wing span at each instant. For these two parameters, four sections at $\mathrm{r} / \mathrm{R}=0.3,0.5,0.7$ and 0.9 are measured, respectively.

The wing kinematics were obtained from the phase average of the reconstruction of the three stroke cycles. Using the right hindwing as an example, Figure 5-3 (a) shows the time course of wing stroke $(\psi)$ and wing deviation $(\phi)$ angles of the hindwing. The wing stroke angle approaches to $76^{\circ}$ at the end of the downstroke. The deviation angle varies around zero with an amplitude of $7^{\circ}$. The wing pitch angles $(\theta)$ at different chord sections are shown in Figure 5-3 (b). The variation of the wing pitch angle showed that the hindwing twisted dramatically during the translational phase in downstroke $(0.1 \leq \mathrm{t} / \mathrm{T} \leq 0.4)$ and in upstroke $(0.7 \leq \mathrm{t} / \mathrm{T} \leq 0.9)$, respectively. In the translational phase, the wing pitch varied largely towards the wing tip. For instance, it changed from $58^{\circ}$ to $123^{\circ}$ and from $46^{\circ}$ to $147^{\circ}$ at $\mathrm{r} / \mathrm{R}=0.3$ and 0.9 , respectively. In addition, the camber deformation was evident at each section and shown in Figure 5-3 (c). The maximum positive camber happened at the mid-chord with the magnitude around $12 \%$ during the downstroke, while the negative camber was $-7 \%$ during the upstroke. It is worth noticing that the maximum camber does not happen in the middle of the downstroke but formed when the wing is about to reversal. 

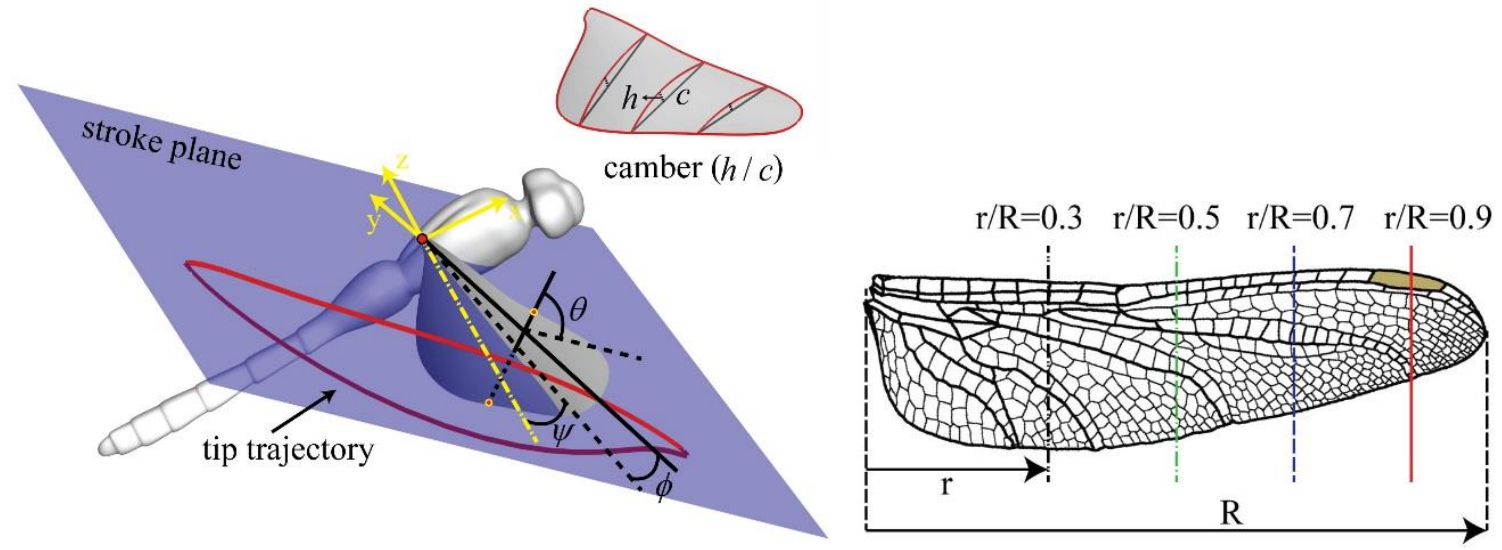

Figure 5-2. Definition of wing orientation angles, wing stroke angle $(\psi)$, wing deviation angle $(\phi)$, wing pitch angle $(\theta)$, and camber deformation $(\mathrm{h} / \mathrm{c}) . \mathrm{r}$ is the distance from wing root to reference point, meanwhile $\mathrm{R}$ is the length from wing root to wing tip.

To investigate the effects of morphing wings, we created two progressively simplified sets of wing kinematics based on the original reconstructed model. In the first simplified model, termed "twist-only", we removed the camber of the wings while retaining the same local instantaneous twist along the wing span. This is achieved by replacing the cambered chord with a straight line connecting the leading and trailing edge. In this simplified model, the chord lengths along the span maintain the same. In the second simplified model, termed "rigid", we further removed the spanwise twist, by replacing the wing with a flat plate of the same instantaneous projected area and same instantaneous wing pitch angle at the mid-wing position. 
(a)

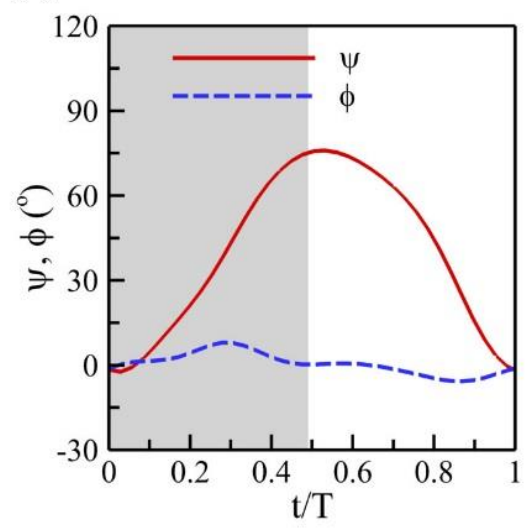

(b)

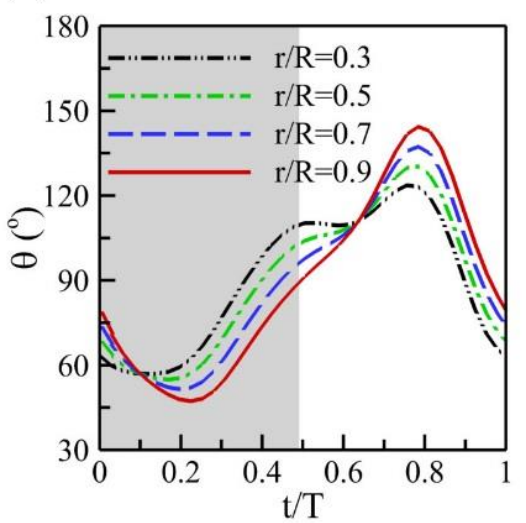

(c)

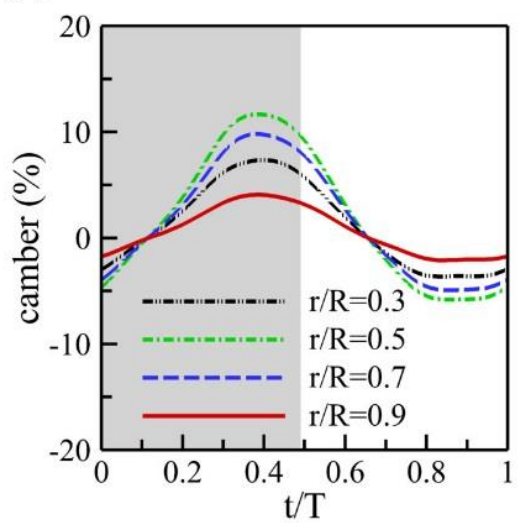

Figure 5-3. Time course of wing kinematics of dragonfly hindwing. (a) Wing stroke angle $(\psi)$ and wing deviation angle $(\phi)$. (b) and (c) are the wing pitch angles $(\theta)$ and camber deromation $(\mathrm{h} / \mathrm{c})$ of different sections along the wing span, respectively. The downstroke period is shaded as a gray color.

\subsection{Aerodynamic Performance}

Using the above-reconstructed wing kinematics, the aerodynamic forces and power required for the flapping motion of three hindwing models were computed using the CFD solver, as shown in Figure 5-4. The stroke plane angle of hindwings were $77^{\circ}$ with respect to the horizontal plane, which indicates that the hindwings flapped with nearly a vertical stroke. Therefore, the positive vertical force was typically created during the downstroke, and slight negative force was formed during the upstroke. The magnitude of the instantaneous vertical of the twist-only wing was smaller than that of the rigid wing, as clearly shown in the middle position of the stroke when the twist angle of the hindwing was largest. The instantaneous vertical force of the original wing with camber variation was similar to that of the twist-only wing during the downstroke. Due to the cruising motion, the force component in the horizontal direction is close to zero, and 
only slight thrust was produced for compensating the body fraction drag during the upstroke. The aerodynamic power consumption in the plot shows that the rigid wing requires more energy for flapping in comparison with the other models. The cycle-averaged values of these parameters are summarized in Table 5-2. From the table, the advantage of wing twist and camber variation was clear in terms of power economy, which is defined as the ratio of the total force to the required power consumption. The results indicate that the power economies for twist-only and rigid wing models are 0.61 and $0.51 \mathrm{~N} \mathrm{~W}^{-1}$, respectively. These values are $3 \%$ and $19 \%$ lower compared with that of the original wing $\left(0.63 \mathrm{~N} \mathrm{~W}^{-1}\right)$.
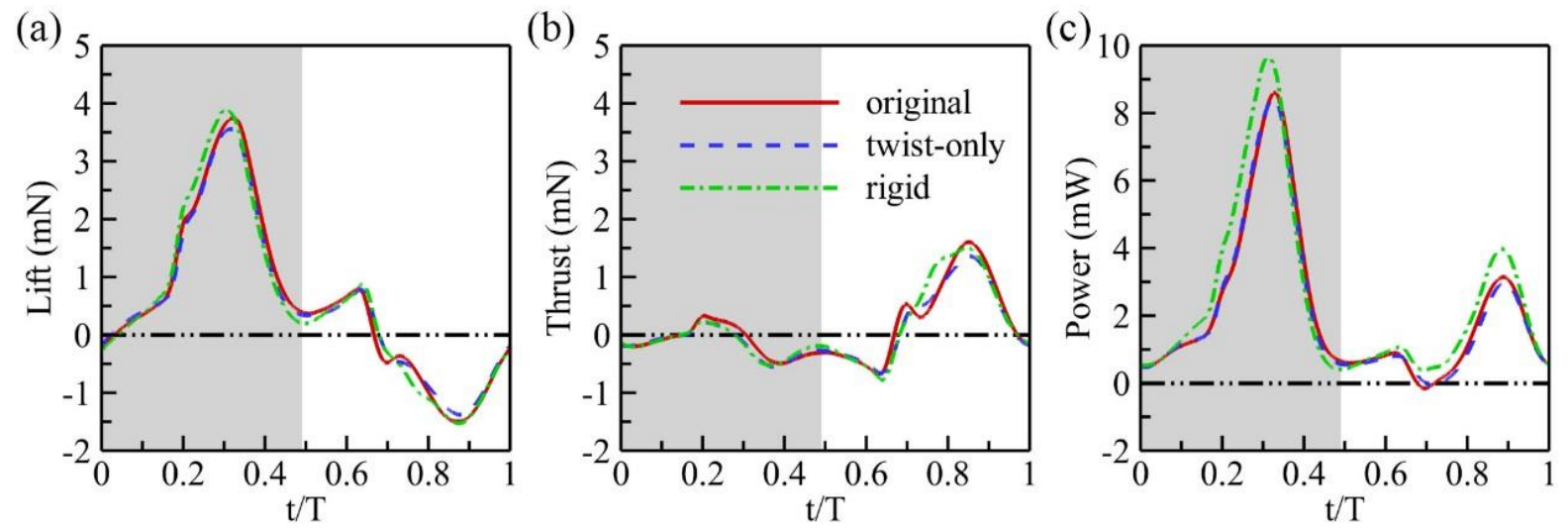

Figure 5-4. Time course of lift (a), thrust (b) and power (c) of the hindwing.

Table 5-2. Comparison of mean lift (L), thrust (T), total force $(\mathrm{F})$, power $(\mathrm{P}), \mathrm{L} / \mathrm{P}$ and F/P of the hindwing during one flapping cycle.

\begin{tabular}{cllllll}
\hline \hline Model & $\mathrm{L}(m N)$ & $\mathrm{T}(m N)$ & $\mathrm{F}(m N)$ & $\mathrm{P}(m W)$ & $\mathrm{L} / \mathrm{P}(N / W)$ & $\mathrm{F} / \mathrm{P}(N / W)$ \\
\hline original & 0.71 & 0.01 & 1.31 & 2.07 & 0.34 & 0.63 \\
twist-only & $0.66(-7 \%)$ & -0.03 & $1.23(-6 \%)$ & $2.02(-2 \%)$ & $0.33(-3 \%)$ & $0.61(-3 \%)$ \\
rigid & $0.67(-6 \%)$ & -0.01 & $1.25(-5 \%)$ & $2.45(+18 \%)$ & $0.27(-21 \%)$ & $0.51(-19 \%)$ \\
\hline \hline
\end{tabular}




\subsection{Vortex Formation}

To gain in-depth insight into the performance of all wing models, the vortex formations for three wing model are shown in Figure 5-5 at the mid-downstroke, at which the wings experience the maximum lift. The leading-edge vortex (LEV) and trailing-edge vortex (TEV) structures at four slides cut at $30 \%, 50 \%, 70 \%$, and $90 \%$ along the spanwise direction from the wing root to the wing tip. The secondary vortex (SV) induced by the interaction between the LEV and wing surface is also evident at each slide cut.

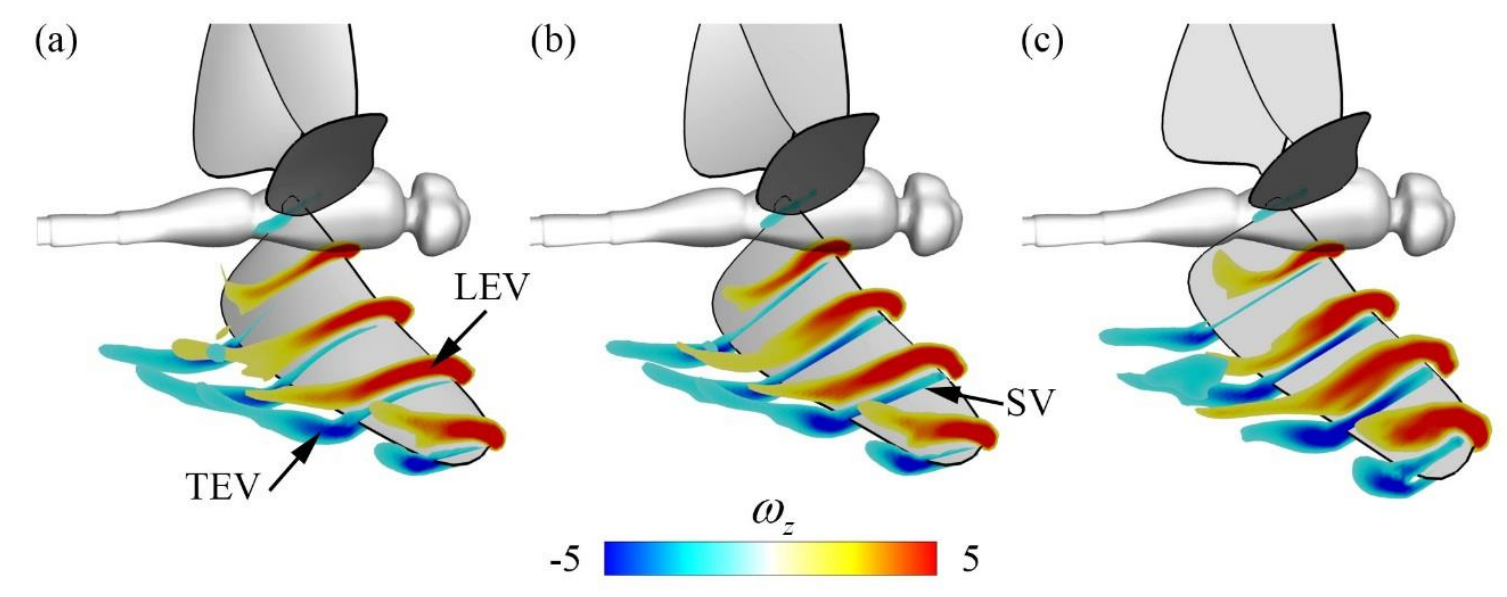

Figure 5-5. Comparison of vortex formation along the wing span at the mid-downstroke $(\mathrm{t} / \mathrm{T}=0.31$ ). (a) Original wing. (b) Twist-only wing (c) Rigid wing. The vortical structure including leading-edge vortex (LEV), trailing-edge vortex (TEV), and secondary vortex (SV) are labeled.

In the comparison of the vortex formation, LEV of the rigid wing was larger than that of the other two model. The LEV of the twist-only wing was similar to the original wing. This 
observation can be further quantified by calculating the LEV circulation along the span. As shown in Figure 5-6, the circulation of the rigid wing sharply increased from the root to the $70 \%$ wing span, and then slightly decreased in the section close to the wing tip. On the contrary, the circulation of the twist-only and original wing models gradually increased from the wing root, and reach to its maximum value at the $70 \%$ wing span. Then, the circulation sharply decreased from the $70 \%$ wing span to the wing tip.

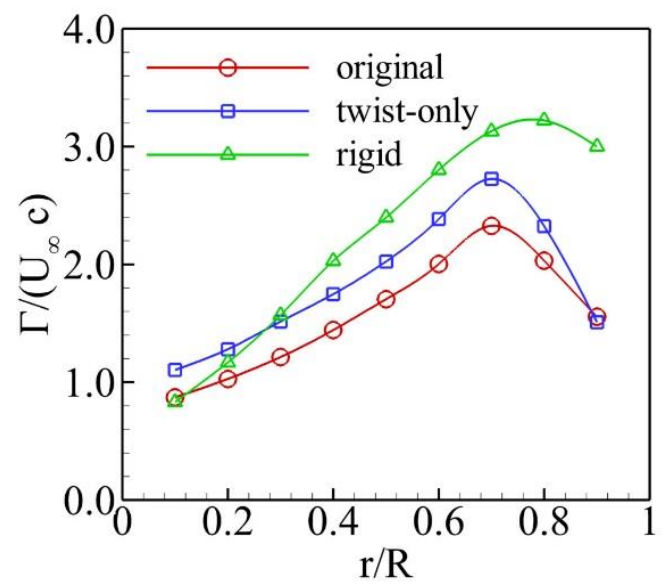

Figure 5-6. Comparison of LEV circulation along the wing span at the mid-downstroke $(\mathrm{t} / \mathrm{T}=0.31)$. The vortex circulation is nondimensionalized by $U_{\infty} \bar{c}$.

Another salient feature that must be noted here is the strength of the secondary vortex variation for different wing models. When the camber and twist deformations were progressively removed, the strength of the secondary vortex was gradually increased and resulted in the detachment of the LEV (Figure 5-5). This phenomenon is in line with the previous observation for a plunging airfoil [123]. As pointed out by Panah et al.[123], the production and entrainment of secondary vorticity is an important mechanism regulating LEV strength because it can provide a dominant sink of LEV circulation. In our cases, the rigid wing model produce a larger sink (secondary vortex) due to the interaction between the wing surface and LEV, and thus form a 
relative stronger LEV compared to the other cases. As a trade-off, the secondary vortex makes the LEV roll-up and detach from the wing surface, and thus reduced the power economy of the rigid wing.

(a)

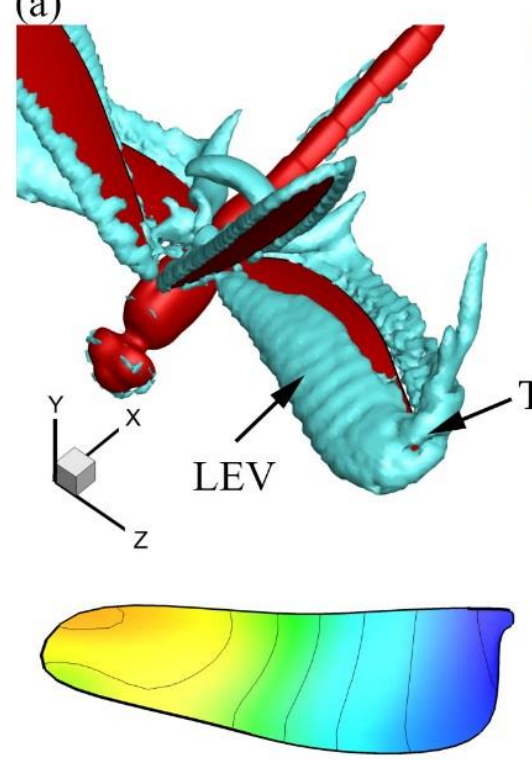

(b)
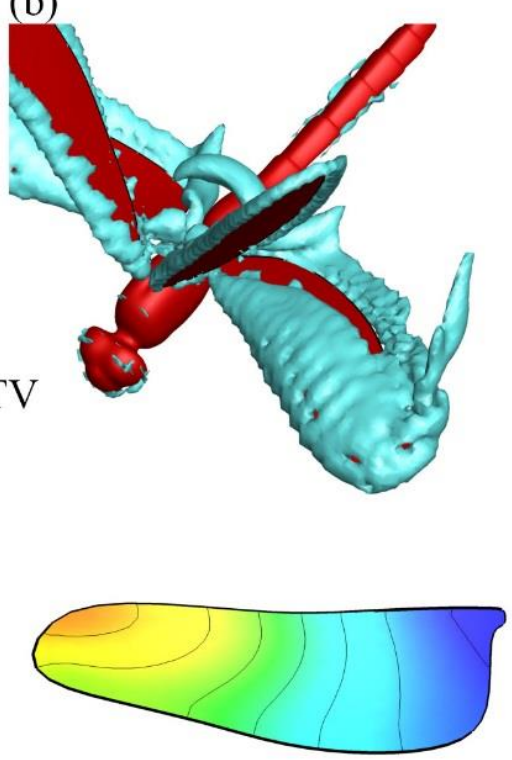

Power $(\mathrm{mW})$ (c)
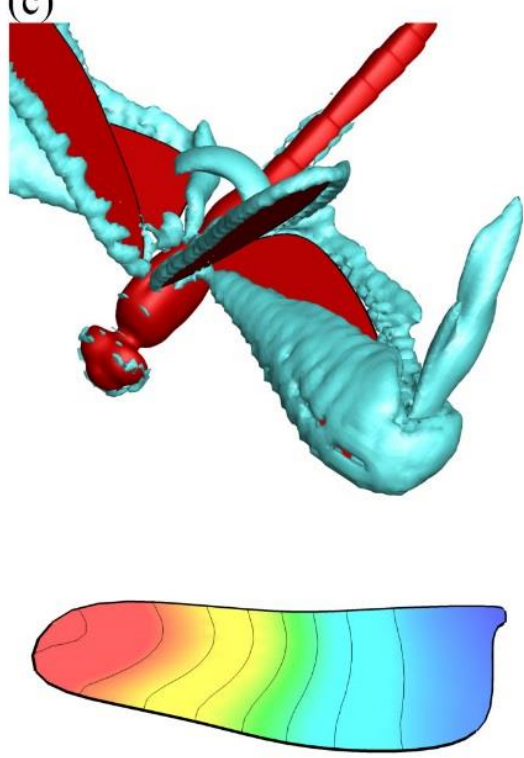

0

Figure 5-7. Comparison of 3-D vortical structure and wing surface aerodynamic power consumption contour of the hindwing at the mid-down stroke $(\mathrm{t} / \mathrm{T}=0.31)$. (a) Original wing model. (b) Twist-only wing model. (c) Rigid wing model.

Figure 5-7 shows the iso-surface of the Q-criterion [94] and aerodynamic power consumption distribution on the hindwing at the mid-downstroke. At this instant, the downward translational velocity of the hindwing reached its maximum value and the LEV occurred on the top surface of the wing, and the LEV near the wing tip was inflected to form the tip vortex (TV). Comparing to the original and twist-only wing model, the rigid wing created a much stronger 
TV. In a comparison of the power consumption contours, the rigid wing model need to consume more aerodynamic power on the wing tip region. This finding can be used to explain our previous observation that the morphing wing can improve the power economy.
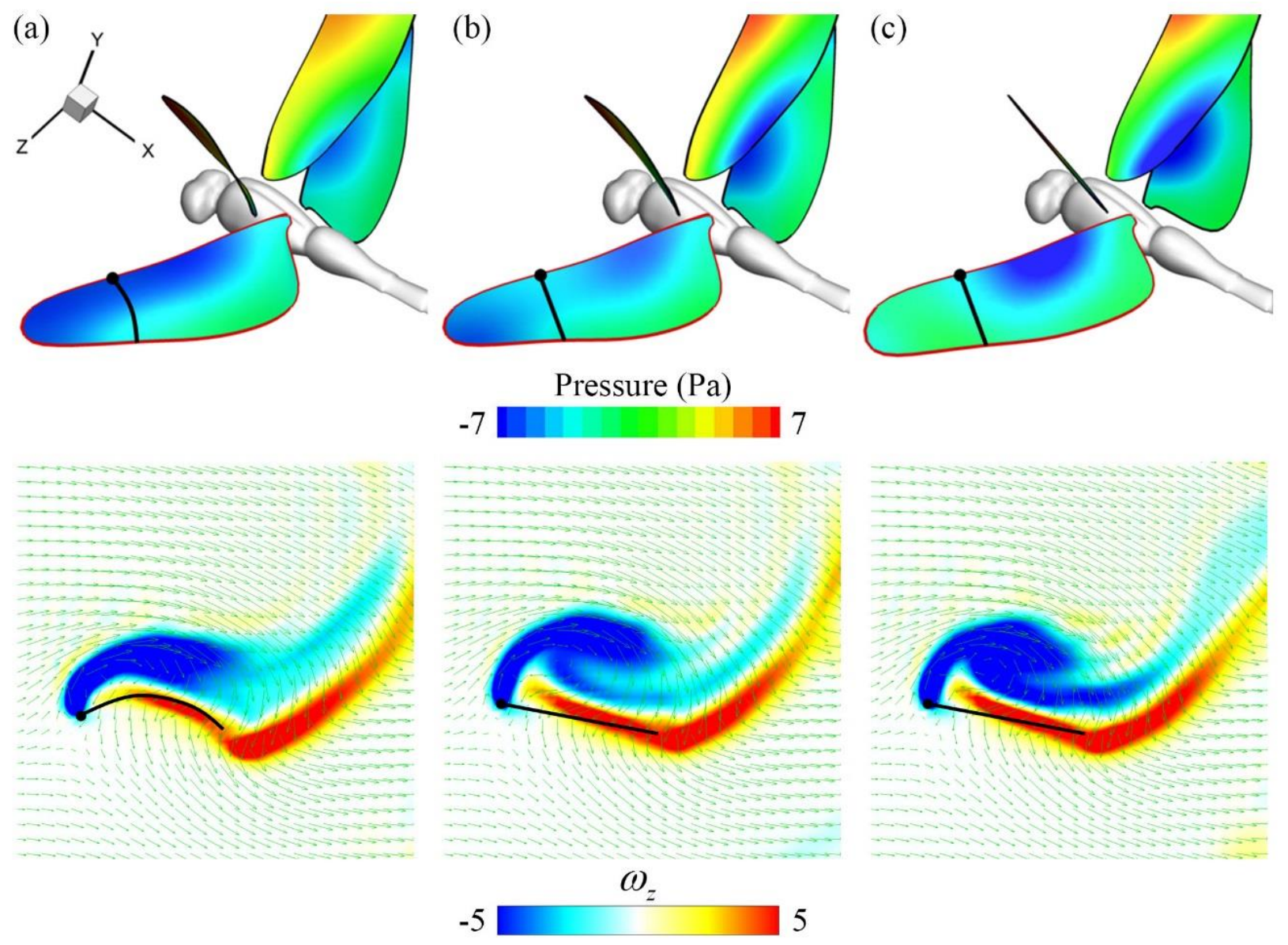

Figure 5-8. Comparison of top surface pressure contours (top row) and the corresponding 2-D flow fields at the $70 \%$ wing span (bottom row) when the hindwing experienced the maximum camber formation (t/T=0.42). (a) Original wing model. (b) Twist-only wing model. (c) Rigid wing model.

To illustrate the camber effects, the surface pressure contours and 2-D flow fields at the $70 \%$ wing span are shown in Figure 5-8, when the hindwing experienced the maximum camber 
formation. For the original wing model, the low-pressure area is along the leading-edge from the wing base to the wing root, and shows no evidence of the leading-edge separation. Due to the locally varying camber, the leading edge is well aligned with the oncoming flow at all the times (Figure 5-8 (a)). In contrast, the rigid wing model present a low-pressure area at the mid-chord section (Figure 5-8 (c)). It implies massive separation on the hindwing. The twist-only wing also shows evidence of separation (Figure 5-8 (b)), but with the low-pressure region extend from the wing root to the wing tip. The 2-D flow fields plotted in the bottom row of Figure 5-8 confirm that the LEV detachment in the two simplified models. Flow reversal is clearly visible over the hindwing for both the twist-only (Figure 5-8 (b)) and rigid (Figure 5-8 (c)) wing models.

\subsection{Chapter Summaries}

In this study, a high-speed photogrammetry system, 3-D surface reconstruction technology and numerical simulations are used to reveal the effects of morphing wings of a cruising dragonfly. Specifically, the flapping morphing wing kinematics of a free-flight dragonfly are measured and quantified first. We then used the reconstructed model to explore the effects of morphing wings, first by removing camber while keeping the same time-varying twist distribution, and second by removing both the camber and the spanwise twist. Our simulation results revealed that the surface deformation can improve the aerodynamic functions in two ways: 1) improving the power economy by preventing the tip vortex bursting; and 2) improving the leading-edge vortex attachment by suppressing the generation of the secondary vortex. As a result, the spanwise twist can boost the aerodynamic efficiency up to $20 \%$, especially during the wing translational phase. 


\section{Aerodynamic Functions of Tandem Flapping Wings in Dragonfly Turning Maneuver}

Clever maneuvers can be commonly observed in insect flight for capturing food and/or avoiding predators. Unlike most other insects such as flies, wasps and cicadas either reduced their hind-wings or mechanically coupled fore and hind wings, dragonflies have maintained two independent-controlled pairs of wings throughout their evolution [50]. Their neuromuscular system allows them to actively change many aspects of wing motion in a single wing, such as the angle of attack, stroke amplitude, and stroke plane. Most previous studies of are focused on the aerodynamics of dragonfly-like tandem wings in steady flight motion. Although the unsteady free flights of dragonflies have also been studies [121,124,125], their works were limited on reporting the wing kinematics and associated flight dynamics. The present effort is meant to fill some of the knowledge gaps in this regard. Specially, a high-speed photogrammetry system and 3-D surface reconstruction technology [87] are used to reveal dragonfly wing kinematics during a turning maneuver flight. The aerodynamic performance is then studied using an in-house immersed-boundary-method-based computational fluid dynamics (CFD) solver. This work aims to investigate the aerodynamic characteristics of forewings and hindwings and its associated forewing-hindwing interaction effects in a turning maneuvering motion.

\subsection{Data Acquisitions}

Dragonflies (Erythemis Simplicicollis) are selected for this study. We collected approximately 110 separated recording of dragonflies' free flying using the images from the calibrated high-speed video apparatus. These videos were then examined carefully and one 
particular recording was selected for that the dragonfly was performing turning maneuver with largest heading rotational angle with three wing beats and for which the image acquisition from all three cameras was of high quality. The morphology of the dragonfly wings is measured when they spread out as in mid-downstroke, using techniques similar to the measurement of hummingbirds. The obtained morphological data are listed in Table 6-1.

Table 6-1. Morphological data for the dragonfly in the current study.

\begin{tabular}{lc}
\hline \multicolumn{1}{c}{ Variable } & Value \\
\hline Body mass (g) & 0.1150 .005 \\
Body length (mm) & 34.5280 .135 \\
Forewing/hindwing length (mm) & $32.3990 .113 / 31.1980 .125$ \\
Forewing/hindwing chord at mid-wing (mm) & $8.5490 .185 / 9.7760 .160$ \\
Flapping frequency (Hz) & $27 \pm 0.5$ \\
\hline \hline
\end{tabular}

The dragonfly free flight is reconstructed using a template-based hierarchical subdivision surface method. The details about this method and its accuracy can be found in Ref.[87,99]. Only brief descriptions are provided here. Taking advantage of marker points we put on the wings before the video shooting and other easily identifiable natural markings, we reconstructed the 3 D deforming wing kinematics at 110 instants during three flapping cycles by measuring the 3-D location of 16 total points on each wing surface from this recording. A 3-D model of each wing is then iteratively mapped onto the marker data, thereby providing a smooth parametric surface representation of the wing in its deformable shape. After that, the wing surfaces were smoothed 
via linear interpolation by holding the coordinates of the measured points. Once the smoothed mesh was obtained, we used Furious interpolation by taking the first 20 terms to interpolate the surface mesh in time to produce a high temporal frequency (1000 time-steps per flapping cycle) input for our CFD solver. Figure 6-1 visualizes the reconstructed motion of dragonfly during turning maneuver at selected instants.

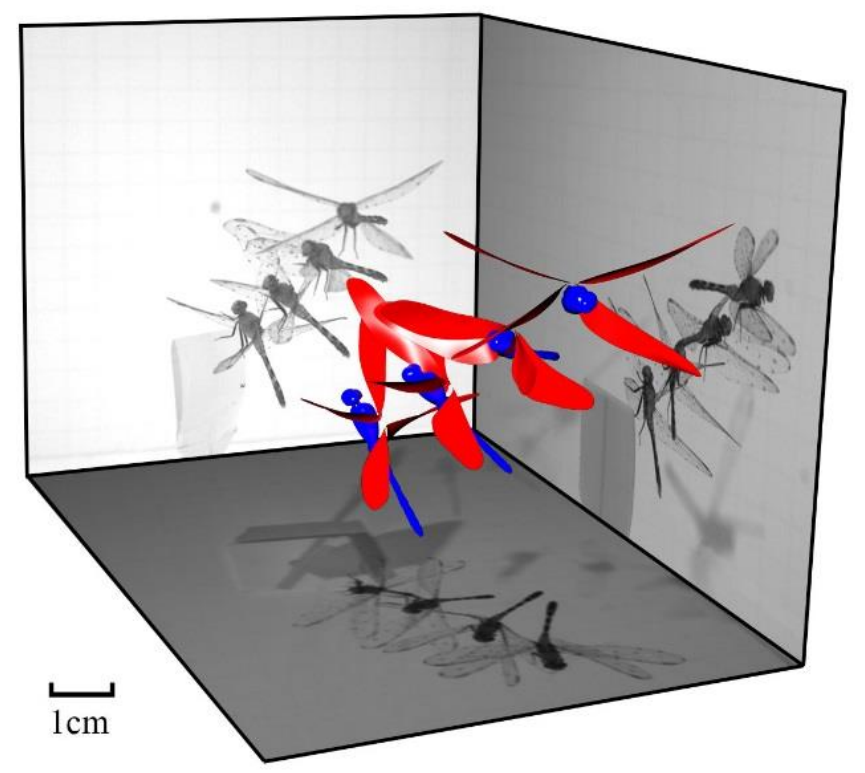

Figure 6-1. Motion reconstruction of dragonfly taking-off maneuver. The side panels show 4 of 110 frames recorded by high-speed videography.

\subsection{Computational Setup}

In the current study, the simulation has been carried out on a non-uniform $233 \times 225 \times 177$ point Cartesian grid. The overall computation domain has a size of $30 \bar{c} \times 30 \bar{c} \times 30 \bar{c}$ in terms of the averaged wing chord length and a cuboidal area around the dragonfly of size $7.0 \bar{c} \times 6.8 \bar{c} \times 5.2 \bar{c}$ has a high-resolution uniform grid $(\triangle \cong 0.031)$ which is designed to resolve the near-wake vortex structures, as shown in Figure 6-2. The stretching grids are applied in all three 
directions from the fine region to the outside boundaries. The time step in this study is 0.001 to ensure stable solutions obtained throughout the simulation. The outside boundary conditions of both velocity and pressure are homogeneous Neumann conditions.

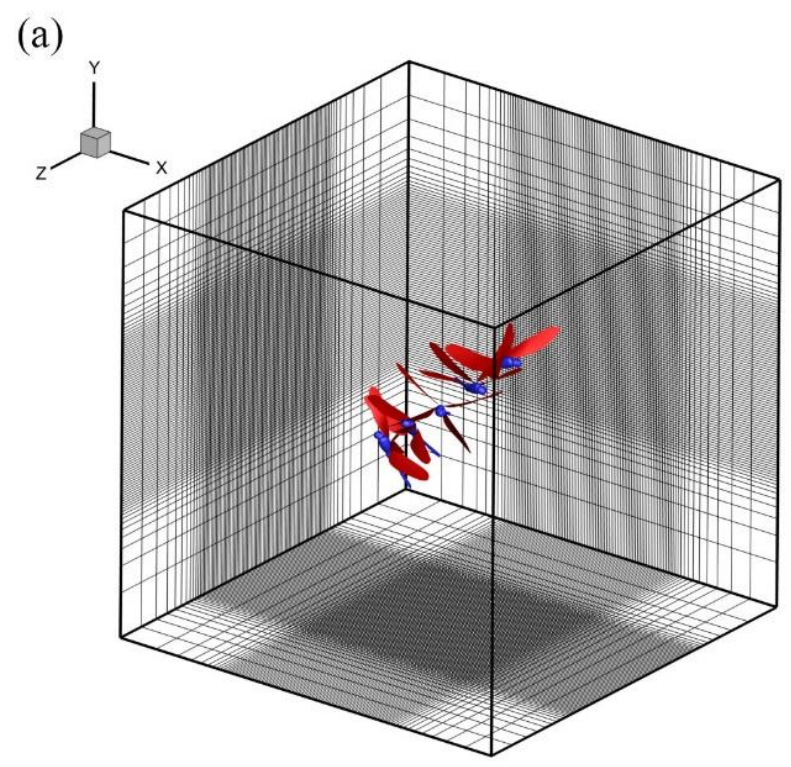

(b)

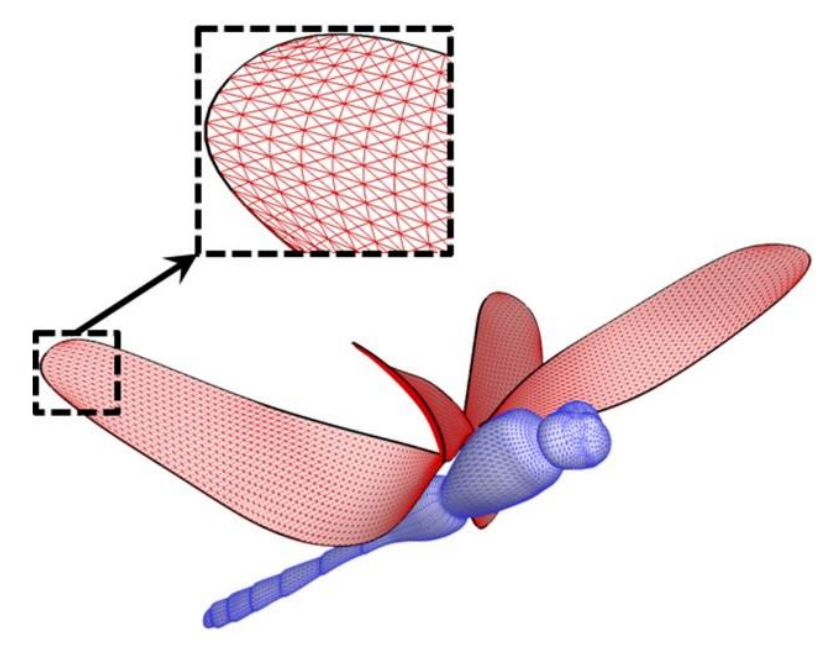

Figure 6-2. (a) Grid and domain employed for the dragonfly maneuver simulations. (b) Highdensity surface mesh with about 3000 triangular elements used to define each wing.

Grid independence is assessed by simulating the same case on two other grids: one with $50 \%$ more and the other with 50\% fewer grid points than the baseline grid. Both of these simulations produce a maximum $1 \%$ difference from the baseline in vertical force. The corresponding differences for the root-mean-square value of horizontal force are $1.79 \%$ and $2.17 \%$ respectively. These results indicate a high level of accuracy in the aerodynamic forces computed on the baseline grid, as well as the flow features that produce these forces. 
The Reynolds number is defined by $\operatorname{Re}=U_{r e f} \bar{c} / v$, where the kinematic viscosity $v$ is around $1.56 \times 10^{-5} \mathrm{~m}^{2} \mathrm{~s}^{-1}$ for air at room temperature $\left(27^{\circ} \mathrm{C}\right)$ and ${ }^{-}$is the mean chord length $(7.7 \mathrm{~mm})$ of the forewings. The averaged tip velocity $\left(U_{r e f}\right)$ of forewings was measured as $2.23 \mathrm{~m} \mathrm{~s}^{-1}$. Based on the measured data in this study, the Reynolds number is about 1100 . The above parameters are all within the range of previous records for free-flight dragonflies [120,121].

\subsection{Body and Wing Kinematics}

Two Cartesian coordinate systems are introduced to described the body kinematics, a global frame $(x, y, z)$ and a body frame $\left(x_{b}, y_{b}, z_{b}\right)$. The plane consisting of $\mathrm{x}$ and $\mathrm{y}$ axes is a horizontal plane, and the z-axis is parallel to the gravity force. The body frame is fixed to the body of the insect, and the origin of the body frame is located at the center of mass of the dragonfly. Body location is directly obtained from the mass center trajectory in the global frame. The time derivatives of the vertical and horizontal components (translational velocity) can be easily calculated. Through the rotation matrix from the global frame to the body frame, the orientation of the insect's body is given by three Euler angles: pitch $\left(\theta_{b}\right)$, roll $\left(\phi_{b}\right)$, and yaw $\left(\psi_{b}\right)$, as shown in Figure 6-3 (a). For more details of the body flight dynamics convention, the readers are referred to dynamics texts written by Etkin et al. [126]. The time course of body kinematics parameters are shown in Figure 6-4(a) and visualized in Figure 6-1.

To describe the motion of the wing kinematics, we select the body-fixed $x_{b}-y_{b}$ plane as the reference plane to measure the wing orientation as shown in Figure 6-4(b). We then quantify wing kinematics based on the $x_{b}-y_{b}$ plane by plotting the time course of three Euler angles: 
wing stroke $(\psi)$, wing deviation $(\phi)$, and wing pitch $(\theta)$ in Figure 6-4(b), which describe the orientation of the wings relative to the wing root.

(a)

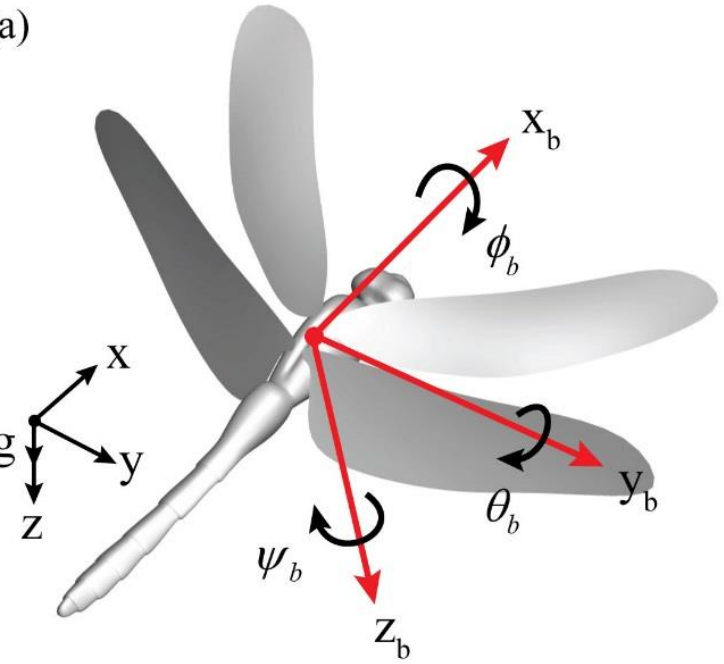

(b)

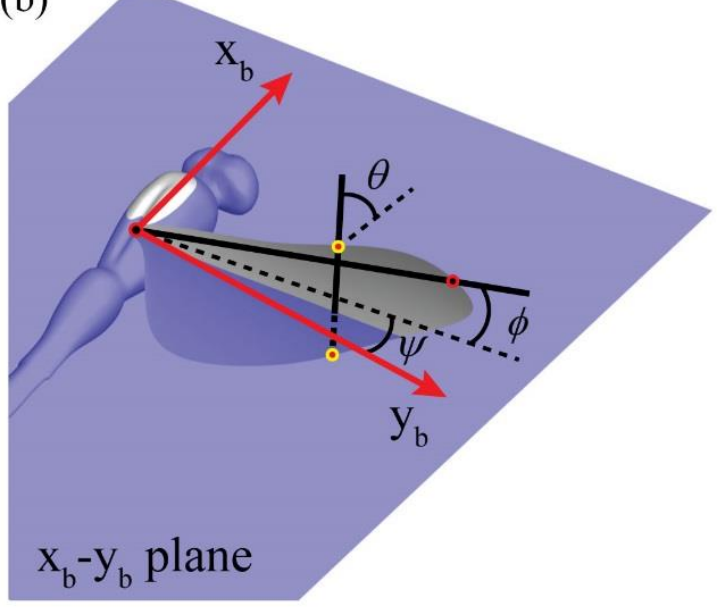

Figure 6-3. Dragonfly body and wing coordinate systems. (a) The body frame $\left(x_{b}, y_{b}, z_{b}\right)$ has its origin at the center of mass. (b) Defination of wing kinematics parametters.

During the flight, the computed body Euler angles show that the body yaw angle magnitude reaches a maximum of $130^{\circ}$ while the magnitude of pitch and roll angles reach a value close to $40^{\circ}$ and $25^{\circ}$, respectively. The negative yaw angle indicates that it is a left-side turn. As the dragonfly's body lift up, the body is gradually pitching down, and associated with rolling in one direction and then rolling back. It is typical a banked turn as defined in previous studies $[124,127]$. Another distinct feature we observed from our recorded videos is that dragonflies always bend their tail towards to the body turning direction. Based on our rough estimation, this will advance the yawing torque generation by change the instantaneous body moment of inertial. The current body orientation angles are calculated based on the vector from the center of mass to the head without considering the tail bending. 
During the maneuver motion, the path velocity in the horizontal direction is around 0.3 $\left(\mathrm{m} \mathrm{s}^{-1}\right)$ when the curve slope closes to zero. In the vertical direction, the path velocity shows an almost linear changing tendency, and the curve slope is approximate $2.1\left(\mathrm{~m} \mathrm{~s}^{-2}\right)$. These values are in a reasonable range comparing with a previous study on dragonfly flight [120].

For performing this $130^{\circ}$ turn and maintaining sufficient vertical acceleration, asymmetrical wing motions are generated between its left and right side. As shown in Figure 6-4(b), there exist significant asymmetries for both forewings and hindwings between the left and right side. The hindwing leads the forewing by about $55^{\circ}$ in the temporal phase, based on the primary Fourier components. Specifically, the wing stroke angle curve of left-side wings shift upward about $11^{\circ} \sim 23^{\circ}$ comparing to the right-side wings, which leads to the inner (left-side) wings have relative large wing stroke angle during the maneuver. The amplitude of wing stroke angle for forewings and hindwings are around $65^{\circ}$ and $80^{\circ}$, respectively. Here the wing stroke angle asymmetry observation is similar to those reported in free turning flight $[124,125]$ although the definition of the wing stroke angle is slight different. The wing deviation angle, on the contrary, did not appear significant difference between the left and right side wings and the amplitude of both forewings and hindwings are about $43^{\circ}$. The wing pitch angles are measured at each $70 \%$ spanwise distance from the wing root. Both forewings and hindwings clearly show asymmetrical pattern between the left and right side. The right-side wings apparently have larger pitch amplitude than the left-side. The magnitude of the former reaches up to $140^{\circ}$, whereas the latter is about $95^{\circ}$. Such asymmetrical wing pitch angle will result in a rather large geometrical angle of attack of the inner (left-side) wings during the downstroke and a small one in the upstroke. In 
which the geometrical angle of attack is the same as wing pitch $(\theta)$ in downstroke and equal $\left(180^{\circ}-\theta\right)$ in upstroke.

(a)

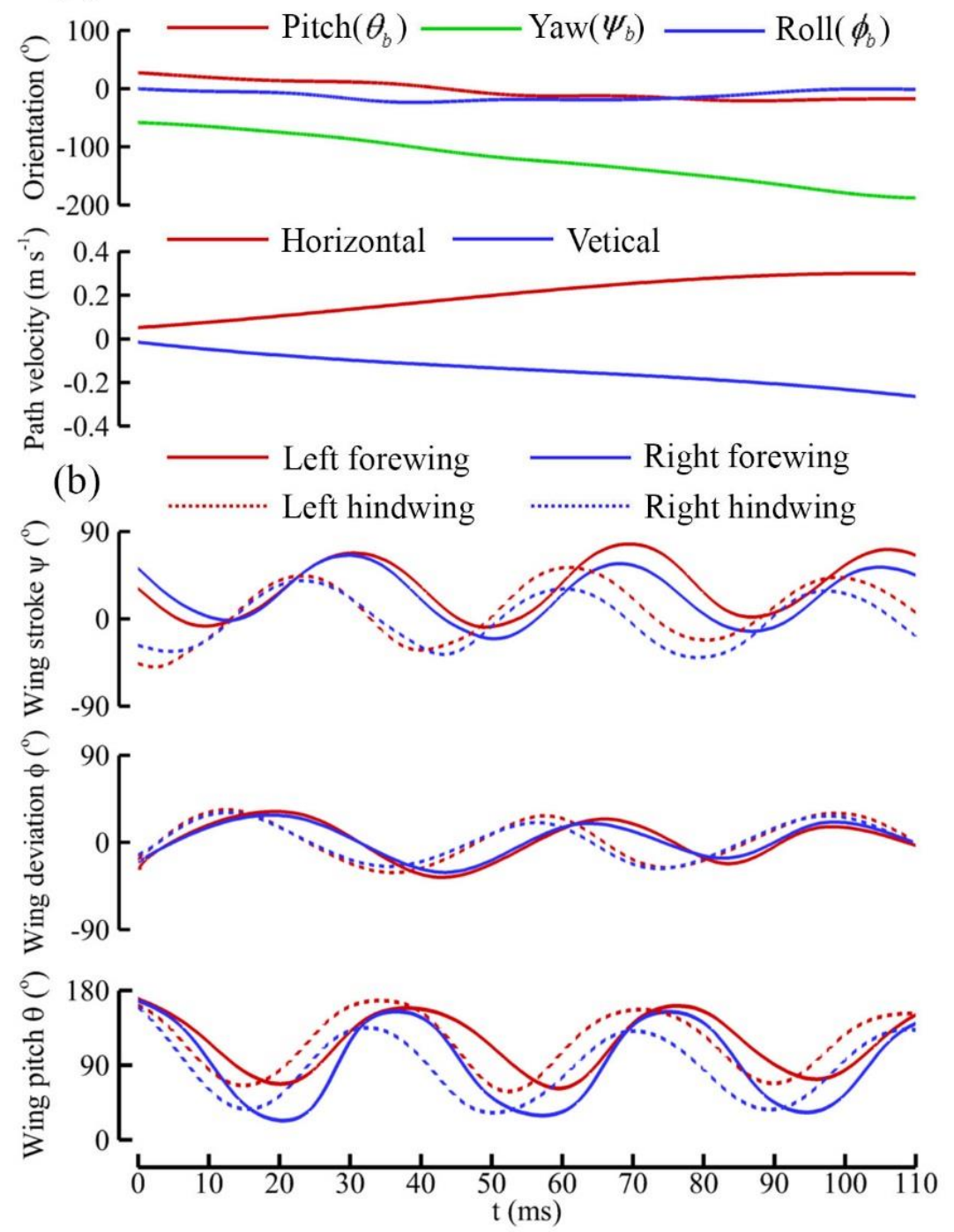

Figure 6-4. Time course of body and wing kinematics of a maneuvering dragonfly. (a) Dragonfly body orientation and path velocity. (b) Wing stroke, deviation, and pitch angles.

Figure 6-5 shows the absolute value of wing tip velocity for both forewings and hindwings. The downstroke of each cycle is shaded by gray color. During the maneuver, the outer wings flap 
faster during each downstroke while the inner wings faster during each upstroke. In addition, the outer (right-side) wings always reverse its flapping direction slight slower during supinating rotation and faster during pronating comparing to the inner (left-side) wings. Combing Figure 6-5 and the wing pitch angle shown in Figure 6-4(b), the inner wings sweep with higher wing pitch angle and experience lower tip velocity during the downstroke. On the other hand, the outer wings flap slower with higher wing pitch during the upstroke. This is analogous to the observation in the study of fruit flies undergoing sideways maneuvers [128]. However, the current study showed that the timing difference appeared in the wing stroke angle rather than wing pitch angle. In addition, the wing stroke angle timing lagging is associated with the asymmetry of wing pitch amplitude.

(a)

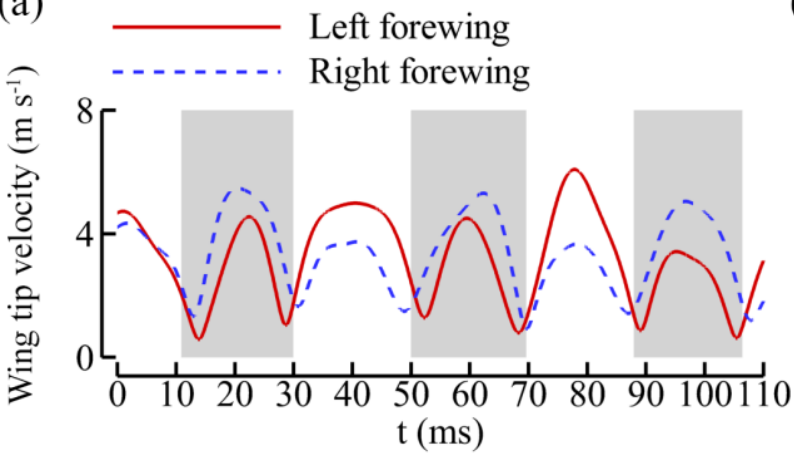

(b)

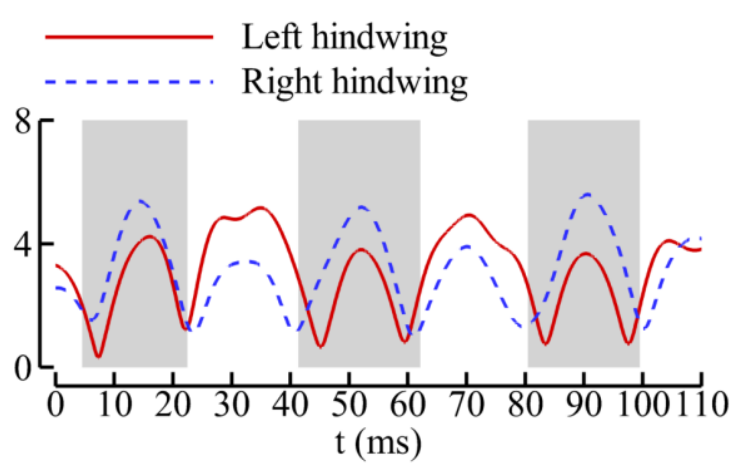

Figure 6-5. Time course of the absolute value of wing tip velocity for forewings (a) and hindwings (b). The downstroke of each cycle is shaded.

\subsection{Aerodynamic Performance}

Using the above reconstructed wing kinematics, the unsteady flow over a maneuvering dragonfly was computed using the CFD solver. The instantaneous aerodynamic forces are obtained through the surface integration of the pressure and shear stress over the wings. These 
forces were resolved in the vertical direction to yield the lift force and the resultant of the force in the horizontal plane along the dragonfly heading direction to yield the forward horizontal force.

(a)

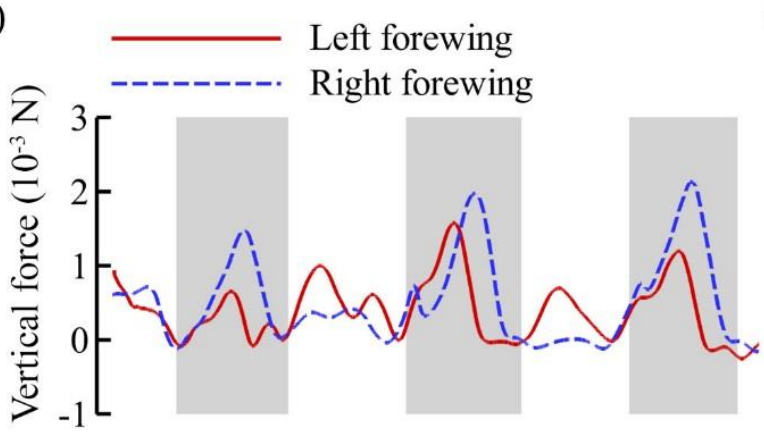

(c)

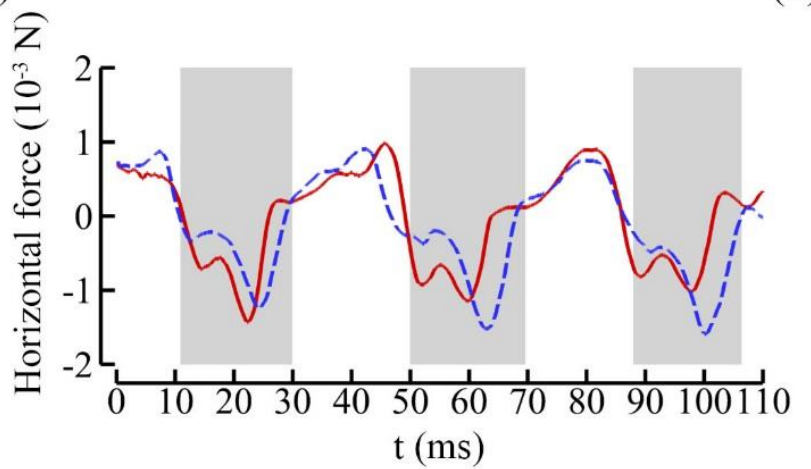

(b)

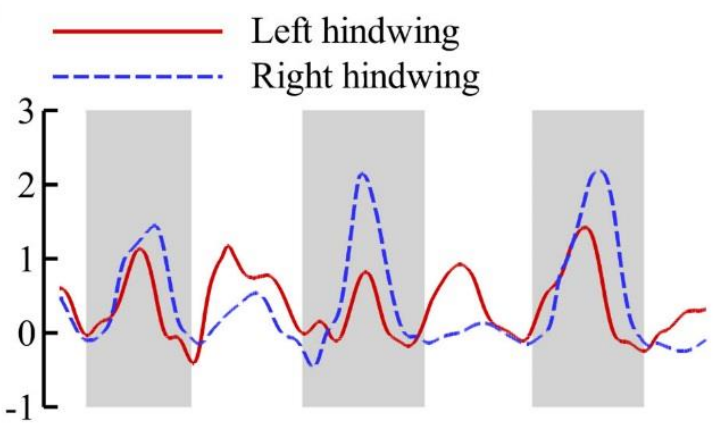

(d)

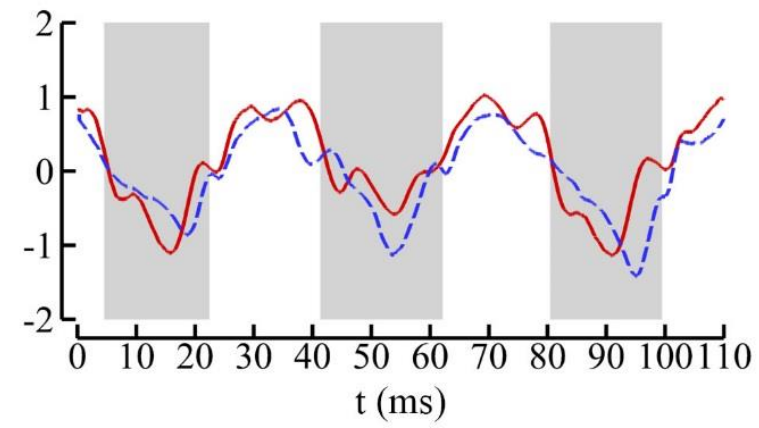

Figure 6-6. Time course of aerodynamic forces of forewings (a, c) and hindwings (b, d), respectively. The downstroke of each cycle is shaded.

The instantaneous vertical forces generated by forewings and hindwings are shown in Figure 6-6 (a) and (b), respectively. It can be easily seen that the vertical force always has a positive value for both upstrokes and downstrokes. The mean vertical force generated by twopair wings is calculated to be $1.36 \times 10^{-3} \mathrm{~N}$. The forewings contribute about $54 \%$ of the total lift, whereas the hindwings accounted for $46 \%$. Considering the weight of the dragonfly was 
$1.13 \times 10^{-3} \mathrm{~N}$, the calculated vertical force from our simulation can generate averaged acceleration in the vertical direction about $2.03\left(\mathrm{~m} \mathrm{~s}^{-2}\right)$. This agrees well with our experimental measurement with $96.7 \%$ accuracy. The integration of the total instantaneous vertical force in Figure 6-6(a-b) showed that for inner wings (left forewing and left hindwing), lift generated during the downstroke and upstroke are 52\% and 48\%, respectively. For outer wings (right forewing and right hindwing), however, $78 \%$ of total lift was generated during the downstroke, whereas the remaining $22 \%$ was generated in the upstroke. In general, the outer wings generated higher lift during the downstroke, whereas the inner wings generated higher lift during the upstroke. This asymmetry vertical force indicates that there are significant unbalance forces to make the dragonfly easy to rolling its body back and forth. This observation is consistent with the body kinematics measurement in the above section.

To manipulate a turn within three wingbeats, the dragonfly also needs to generate sufficiently unbalanced force on the horizontal plane. Figure 6-6(c-d) are the force experienced by the dragonfly along its heading direction, with negative and positive values indicating drag and thrust, respectively. It showed that the dragonfly experience drag in the downstroke and produced thrust in the upstroke. For the forewings, the drag and thrust force magnitudes are about $1.53 \times 10^{-3} \mathrm{~N}$ and $0.98 \times 10^{-3} \mathrm{~N}$, respectively, resulting in a ratio of 1.56 . By contrast, the drag and thrust force magnitudes of hindwings are comparable, in which they are $1.03 \times 10^{-3} \mathrm{~N}$ and $0.97 \times 10^{-3} \mathrm{~N}$, respectively. It is also worth noting that the drag peaks of inner wings appear earlier than the outer wings, especially for the forewings. This timing difference between drag force generated by the left and right wings can be related to the wing pitch angle change during reversal. 
The torques experienced by the dragonfly during the maneuver is computed about the body mass center. The time courses of the aerodynamic torques of the wings are plotted in Figure 6-7 for roll, pitch and yaw torques individually. To get a better understand of the torques generated during the turn, Figure 6-8 shows the mean value of torques for each half cycle.

(a)

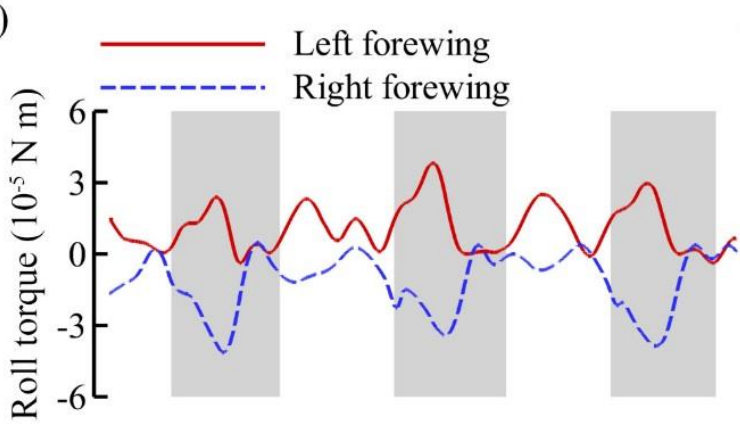

(c)

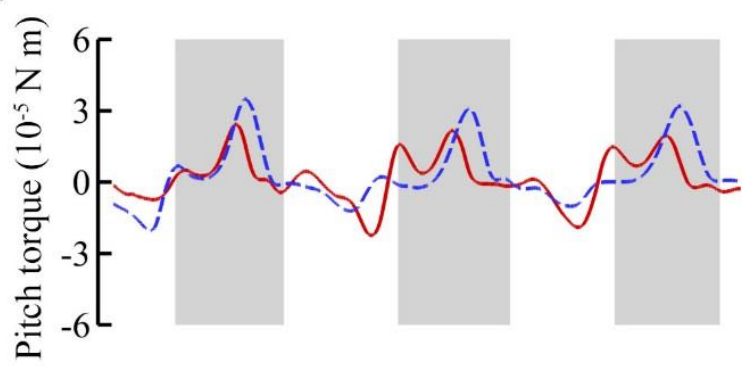

(e)

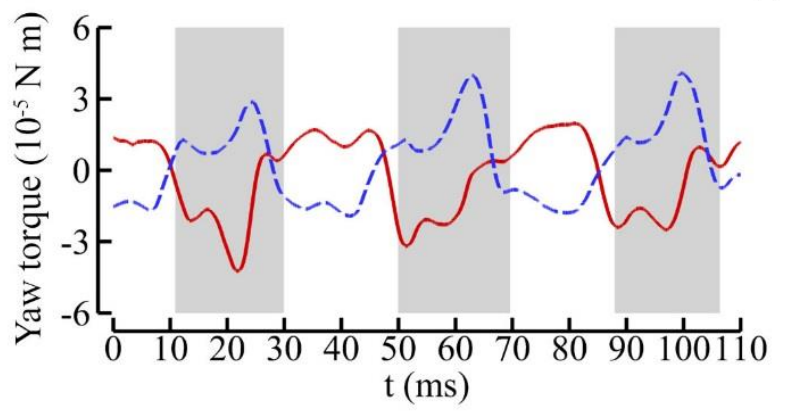

(b)

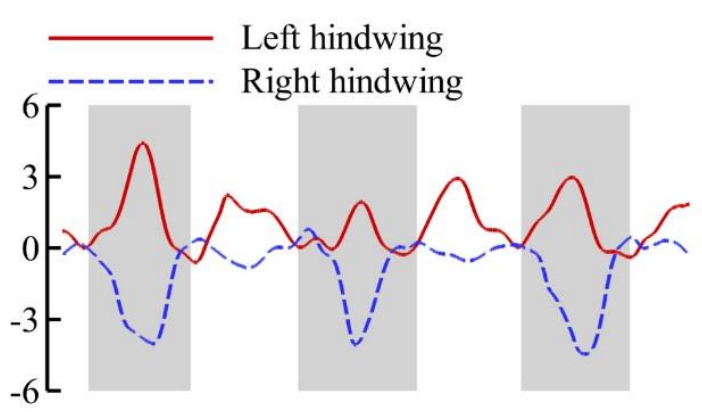

(d)

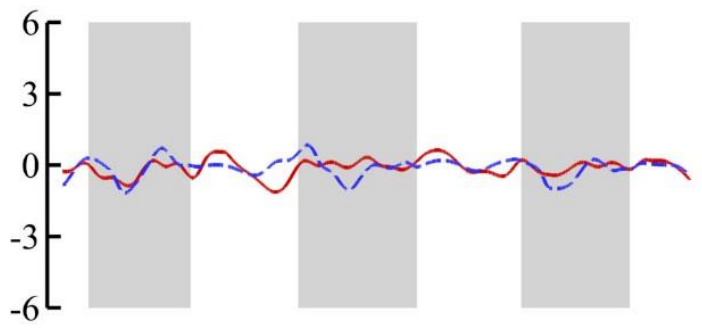

(f)

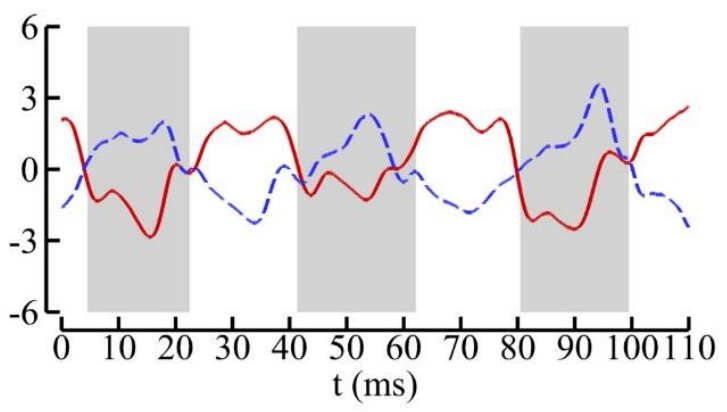

Figure 6-7. Time course of aerodynamic torques of forewings (a, c, e) and hindwings (b, d, f), respectively. 
In Figure 6-7 (a-b), the roll torques generated by left- and rigid- side wings showed opposite signs during each entire stroke and canceled with each other. The roll torque peaks of the outer (right-side) wings, in general, higher than the inner (left-side) wings during the downstroke, and lower than the inner wings during the upstroke. This leads to the half-cycle-averaged resultant roll torques (Figure 6-8) present negative and positive values during the downstroke and upstroke, respectively. The comparable roll torque magnitudes of two-pair wings indicate that both forewings and hindwings contribute to the roll torque generation. Unlike roll torque, the pith torque generation is dominated by forewings. As shown in Figure 6-7 (c-d), the pith torque generation of forewings is approximately one order of magnitude larger than that of hindwings. For the yaw torque generation, the left- and right-side wings also produce torques in the opposite direction and alter signs for each half-stroke (Figure 6-7 (e-f)). The resultant yaw torques averaged by each half cycle (Figure 6-8) show that the majority of yaw torque is also produced by the forewings other than hindwings.

(a)

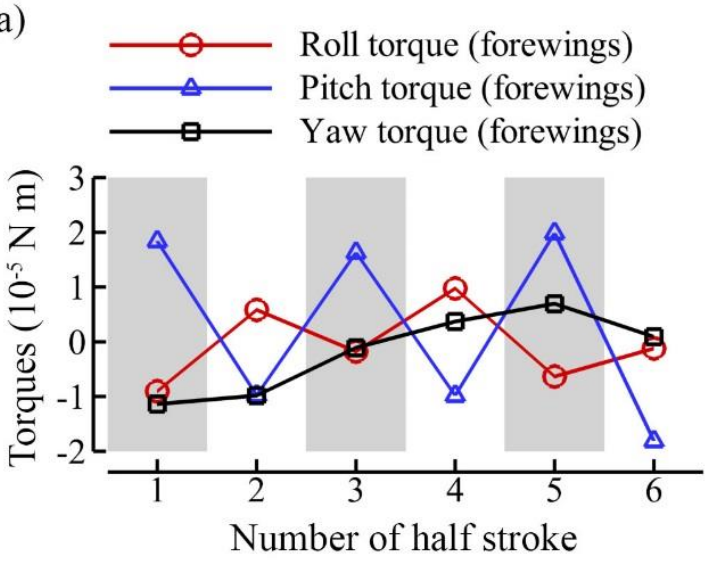

(b)
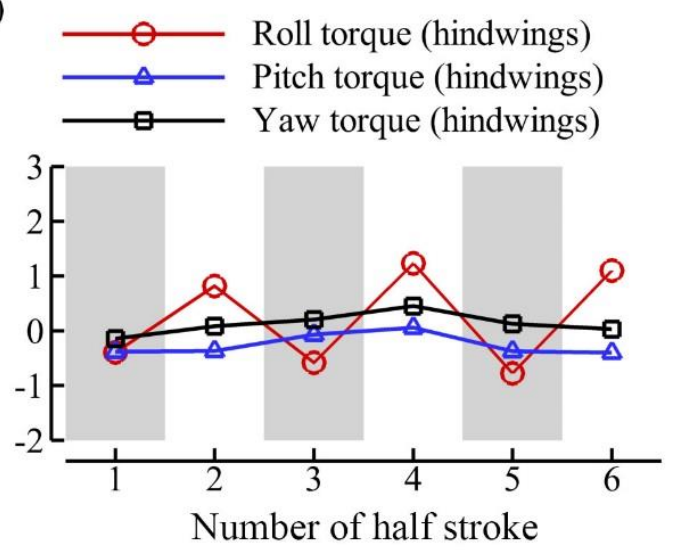

Figure 6-8. Mean values of torques varying with each half cycle of forewings (a) and hindwings (b), respectively. 
Based on computed instantaneous aerodynamic forces and wing velocities, the aerodynamic power consumption of two-pair wings is calculated and normalized by the flight muscle mass. Figure 6-9 provides instantaneous specific aerodynamic powers of forewings and hindwings. During the maneuver, the aerodynamic power of each wing is always positive, although its value is quite small near stroke reversal. For forewings, the averaged power consumption of left- and right-side wings are 34.0 and $45.0 \mathrm{~W} \mathrm{~kg}^{-1}$, respectively, during the downstroke. By contrast, during the upstroke, the left-side wing consumes higher power than right-side wing, in which specific aerodynamic power are 32.3 and $19.1 \mathrm{~W} \mathrm{~kg}^{-1}$, respectively. The hindwings also present the similar pattern as forewings. During the downstroke, the power consumption of left and right hindwings are 26.2 and $42.5 \mathrm{~W} \mathrm{~kg}^{-1}$. During the upstroke, however, the specific aerodynamic power of left hindwing is $38.2 \mathrm{~W} \mathrm{~kg}^{-1}$, which is approximately twice of the right hindwing.

(a)

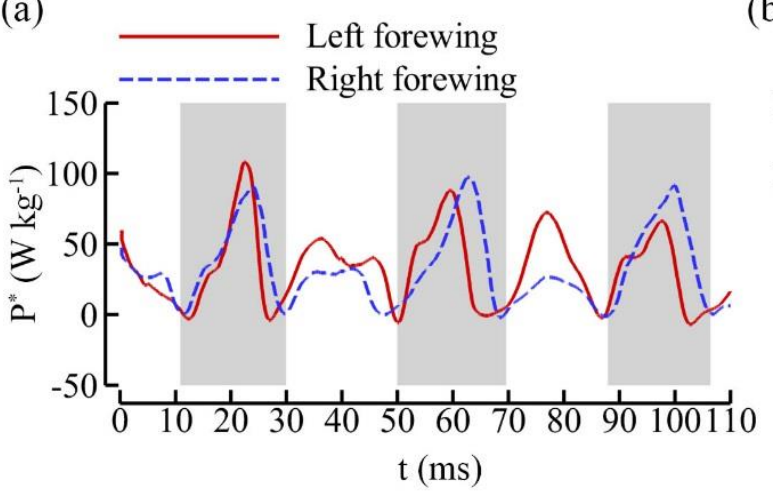

(b)

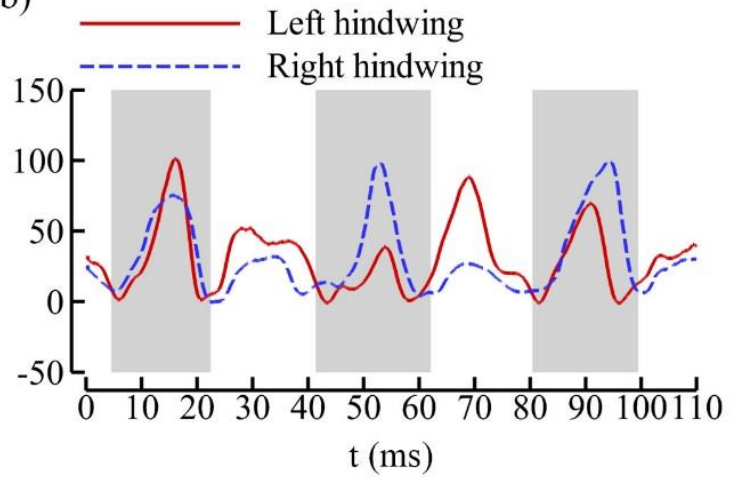

Figure 6-9. Time course of specific aerodynamic power consumption of forewings (a) and hindwings (b), respectively.

The wing surface pressure contours are used to understand the force distribution on the wing surface. The aerodynamic pressure difference between top and bottom surface is projected in vertical and horizontal directions to indicate the horizontal and vertical force distributions over 
the wing surface. Figure 6-10 shows the time-averaged pressure distribution that is normalized by the wing load (the ratio of the dragonfly weight to the total wing surface area). From Figure $6-10$, it can be seen that most of the forces are generated in the region from $70 \%$ spanwise distance to the wingtip, especially for the portion close to the leading-edge. For both forewings and hindwings, the maximum vertical and horizontal forces are all generated at the tip region. By comparing the pressure between the left and right side wings, forewings present more asymmetric distribution than hindwings. This implies that the forewings play a more significant role than the hindwings in manipulating maneuver flights.

(a)

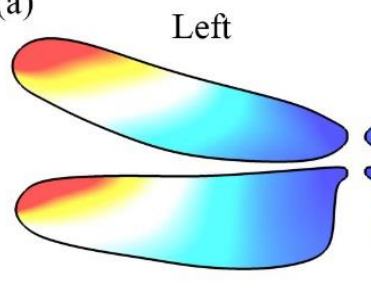

0

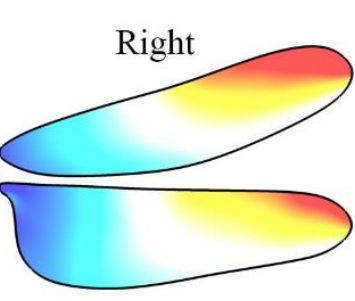

5 (b)

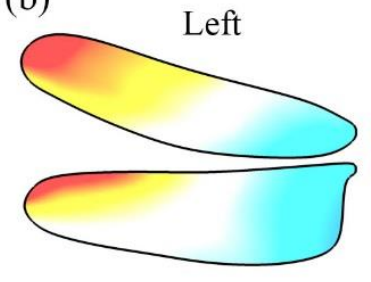

0

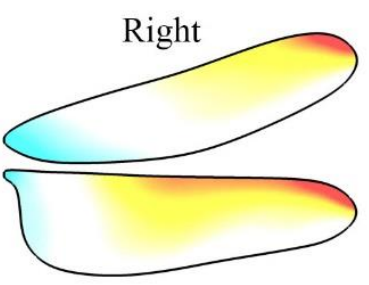

5

Figure 6-10. Time-averaged surface pressure distribution projected on the vertical direction (a) and horizontal direction (b).

\subsection{Vortex Formation}

Figure 6-11 shows the time sequence of the 3-D flow field in the early stage of the turning motion, which is identified by plotting the iso-surface of the Q-criterion [94]. The key feature observed here is the presence of vortex rings and wing-wing interactions. 

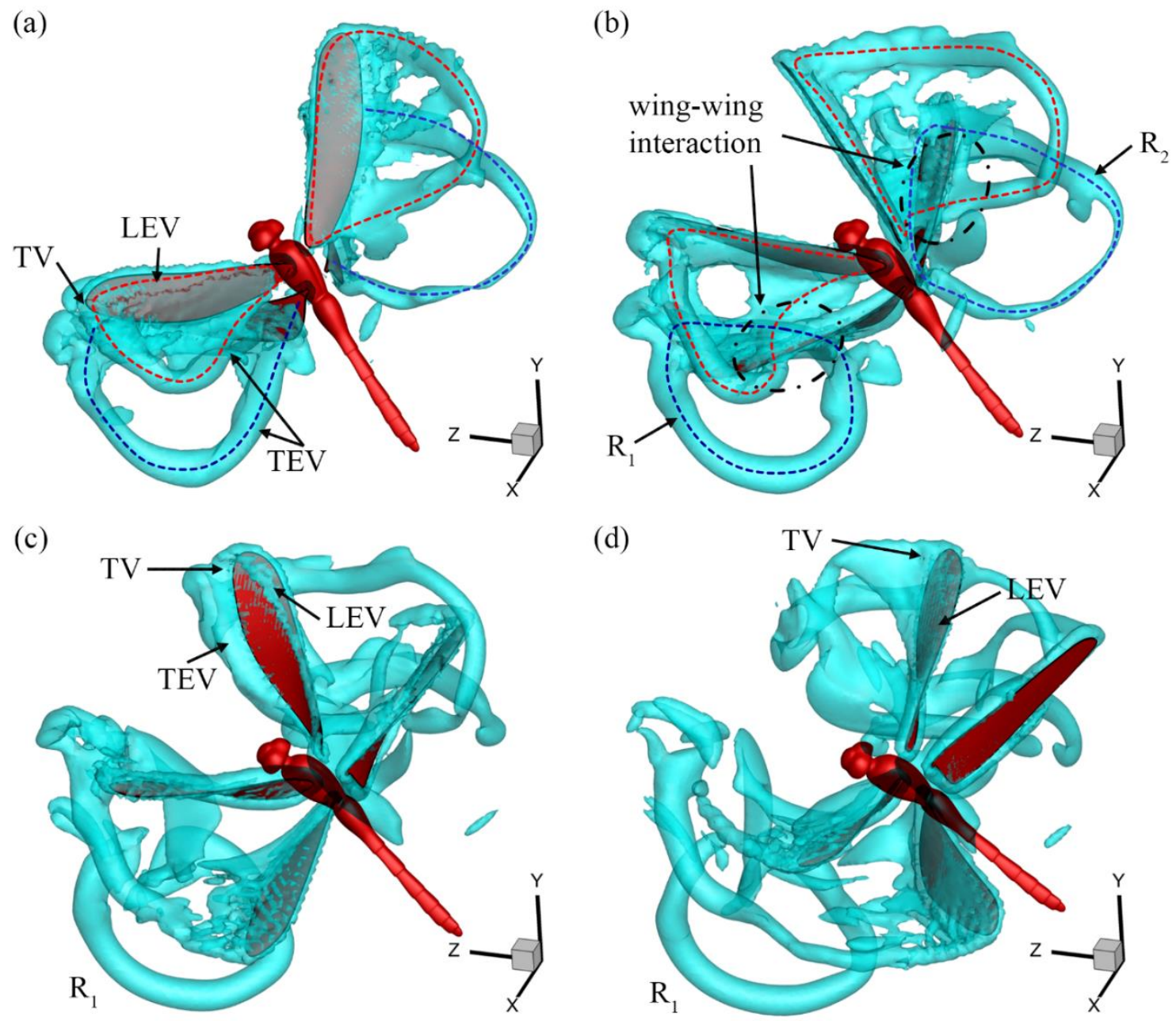

Figure 6-11. 3-D vortex structures in the flow during the early turning motion, where the time stamp from (a) to (d) is $23,29,35$ and $40 \mathrm{~ms}$. The vortex loop from the downstroke is marked by dashed lines.

For each wing, a leading-edge vortex (LEV) is developed and grows stronger remaining stably attached to the wing during the downstroke. As the wing translation, the LEV, the tip vortex (TV) and the shed trailing-edge vortex (TEV) are connected and form a vortex loop, as shown in Figure 6-11 (a). The vortex loops formed by forewings and hindwings are marked by dashed lines in red and blue color, respectively. As the hindwings flapping upward, distinct fully developed vortex rings are gradually shed into the flow field from the trailing-edge of wings, and 
which are identified as $R_{1}$ and $R_{2}$ (as shown in Figure 6-11 (b)). Because of the phase relationship between fore- and hindwing, when the forewings reach the end of its downstroke, the hindwing already start to move upward. The upward moving hindwings interact with the wake formed by the forewings. This flow feature is termed as wing-wing interaction in previous 2-D and 3-D tandem wings studies [54,129]. In addition to the wing-wing interaction, during the upstroke, the wings will catch its own wakes produced by the preceding downstroke and disrupts this loop through the wing-wake interaction, and form stronger TV and TEV, as shown in Figure 6-11 (c). During the maneuver motion, the asymmetrical vortex formation between left and right side is also distinct. By comparing the iso-surface plot of $R_{1}$ and $R_{2}$, the inner (left) side vortex ring is stronger than the outer (right) side one. This asymmetrical phenomenon also makes the shed vortex rings tilted and distorted. By interacting with the vortex loop formed by other wings and their previous shed vortex rings, the wake becomes to more complicated, as shown in Figure 6-11(d).

\subsection{Forewing-Hindwing Interaction}

Wing-wing interaction is a unique feature applied by two-pair wings flyers. During the flapping motion, the forewing and hindwing in each side perform as a tandem wings. In order to examine the effects of forewing-hindwing interaction in dragonfly maneuver flight, two additional simulations, one with forewings only (FO) and the other with hindwings only (HO), are carried out and used to compare with two-pair wings (TPW) simulation results. Figure 6-12 Figure 6-14 compare the time courses of forces and power between two-pair wings simulation (solid red line) and the isolated single-pair wings simulation (dashed blue line). The differences 
between the two lines in each plot show the interaction effects. In order to obtain a quantitative comparison, Table 6-2 lists the mean force generation and power consumption of above cases.

(a)

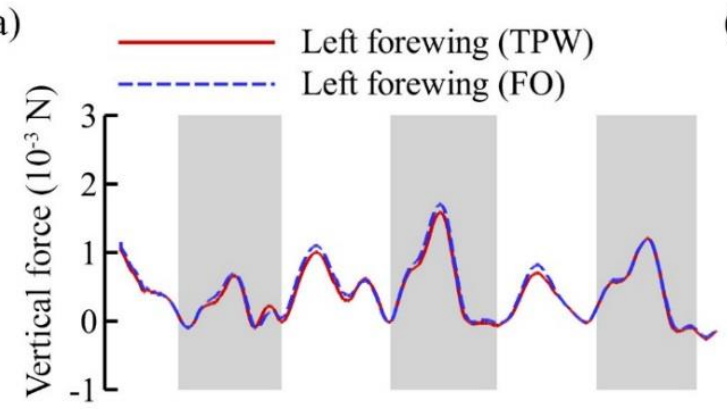

(c)

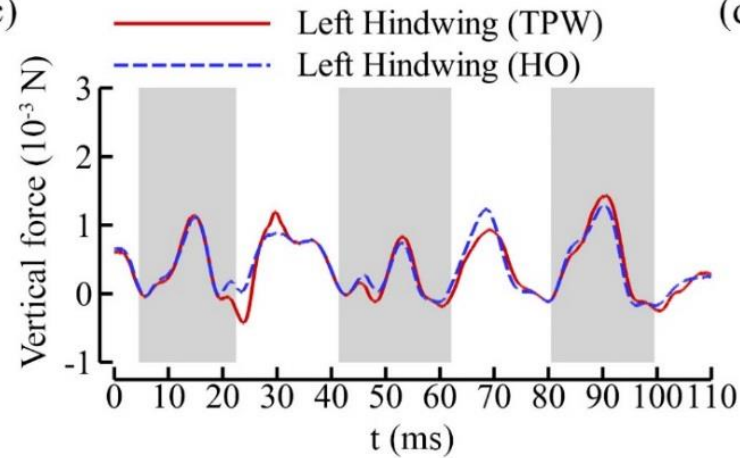

(b)

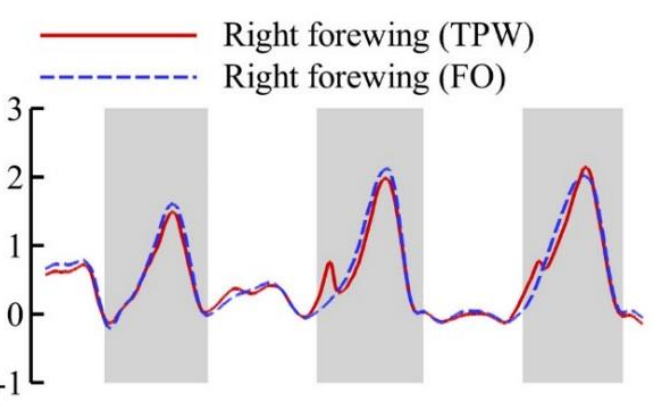

(d)

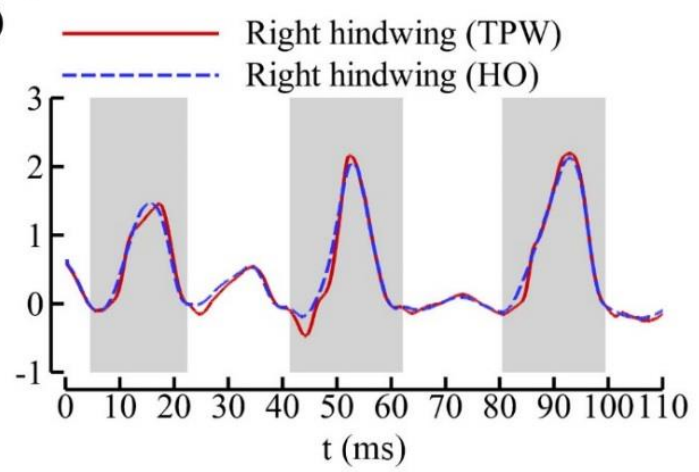

Figure 6-12. Comparison of instantaneous vertical force between the two-pair wings simulation and single-pair wings simulation. (a) left-forewing. (b) left-hindwing. (c) right-forewing. (d) right-hindwing.

As shown in Figure 6-12, the case with two-pair wings present vertical force increasement at certain periods and force reduction at some other period. For the forewings, the interaction effect reduces the mean vertical force by about $6 \%$ of that of the isolated forewings; for the hindwings, the reduction is about $11 \%$ of that of the isolated hindwings. The wing-wing interaction also reduces the force component in the horizontal direction (as shown in Figure 6-13). Comparing to the isolated single-pair wing simulation, the interaction effects reduce approximate 3\% of mean horizontal force for both downstroke and upstroke. In general, the case 
with isolated single pair wings could generate slight higher forces than two-pair wings. This observation is consistent with previous studies. For instance, Wang and Sun $[58,61]$ studied the forewing-hindwing interaction using computational models at Reynolds number in an order of $10^{3}$. At hovering, the resultant forces generated by wings reduce by $8-15 \%$ due to the existence of wing-wing interaction. For forward flight condition, the force reductions gradually decrease as the increasement of the advance ratio. As proposed by Lehmann [64], this force reduction of the interaction effect is due to the decrement of the angle of attack between each wing and the local fluid.

(a)

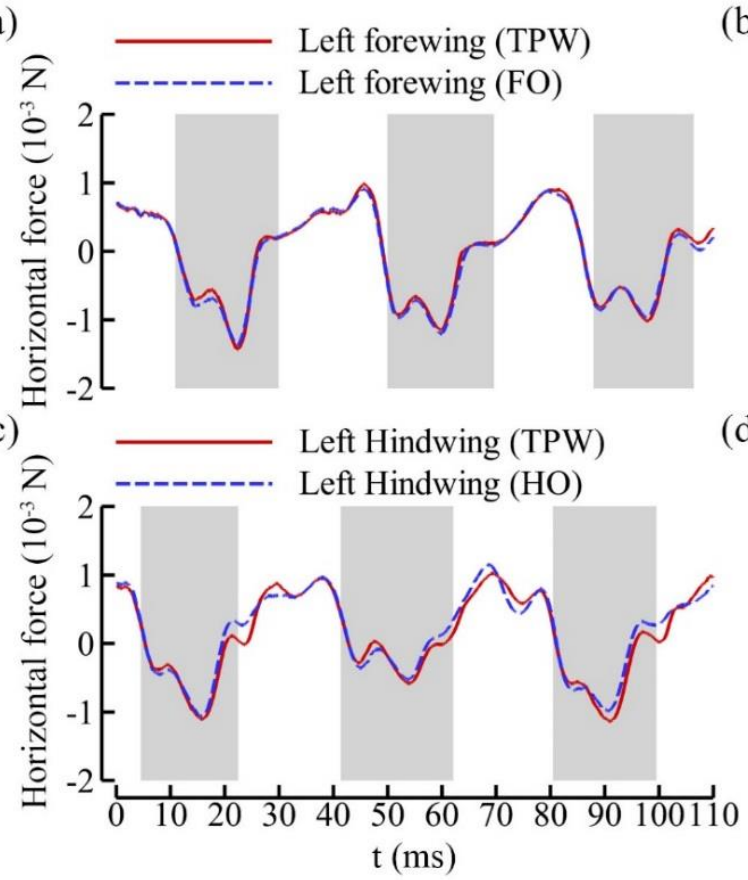

(b)

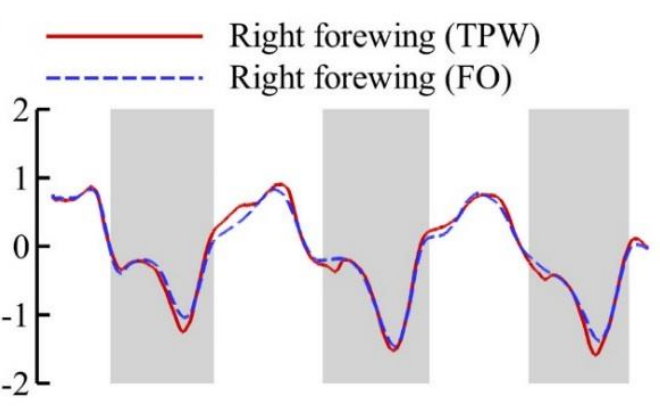

(d)

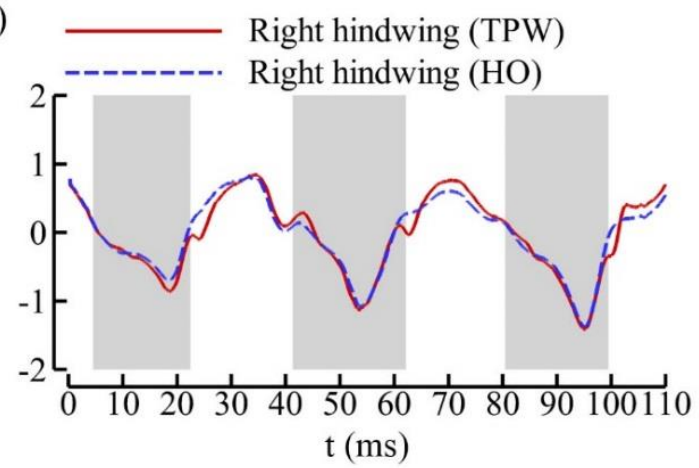

Figure 6-13. Comparison of instantaneous horizontal force between the two-pair wings simulation and single-pair wings simulation. (a) left-forewing. (b) left-hindwing. (c) rightforewing. (d) right-hindwing. 
Despite the force reduction, the interaction effect can save the aerodynamic power consumption. Figure 6-14 provides a comparison of the specific aerodynamic power between the two-pair wings simulation and the single-pair wings simulations. For forewings, interaction effect reduces the power about $3 \%$ of that of the isolated forewings; for hindwings, aerodynamic power expenditure saves up to $11 \%$ of that of the isolated hindwings. This observation is in line with previous experimental studies of tandem robotic wings [63,64]. The power economy of the interaction effect is achieved by recovering energy from the wake wasted.

(a)

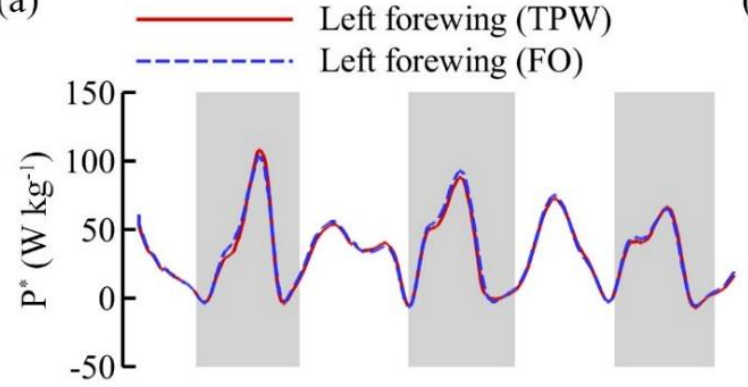

(c)

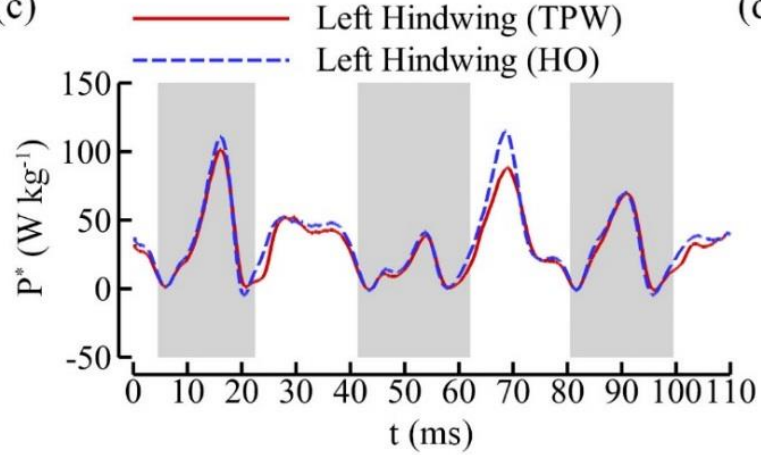

(b)

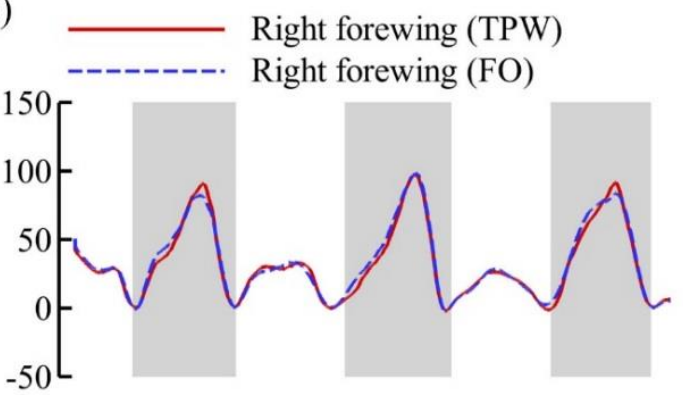

(d)

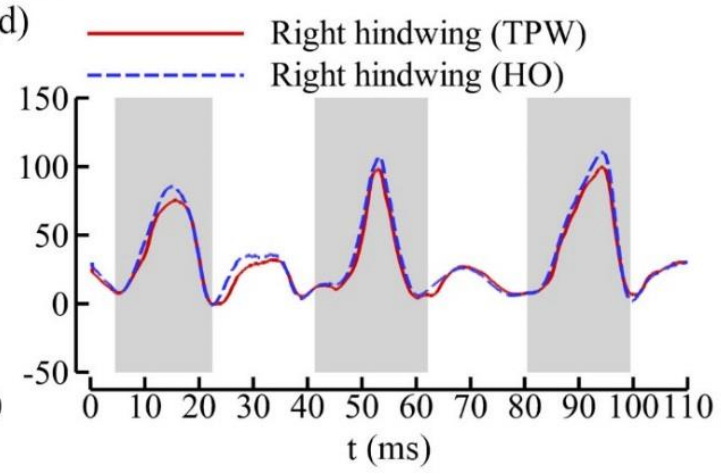

Figure 6-14. Comparison of instantaneous specific power between the two-pair wings simulation and single-pair wings simulation. (a) left-forewing. (b) left-hindwing. (c) right-forewing. (d) right-hindwing. 
Table 6-2. The effects of forewing-hindwing interaction on the mean aerodynamic forces and power.

\begin{tabular}{cccccccccc}
\hline \hline & \multicolumn{2}{c}{ Left forewing } & \multicolumn{2}{c}{ Right forewing } & \multicolumn{2}{l}{ Left hindwing } & \multicolumn{2}{l}{ Right hindwing } \\
\cline { 2 - 9 } & FO & TPW & FO & TPW & HO & TPW & HO & TPW \\
\hline Vertical force $\left(10^{-3} \mathrm{~N}\right)$ & 0.34 & 0.32 & 0.45 & 0.42 & 0.34 & 0.31 & 0.36 & 0.32 \\
Horizontal force & -0.51 & -0.51 & -0.63 & -0.62 & -0.38 & -0.38 & -0.46 & -0.45 \\
during downstroke $\left(10^{-3} \mathrm{~N}\right)$ & & & & & & & & \\
$\quad$ Horizontal force & 0.54 & 0.54 & 0.48 & 0.47 & 0.69 & 0.69 & 0.47 & 0.46 \\
during the upstroke $\left(10^{-3} \mathrm{~N}\right)$ & & & & & & & & \\
Specific power $\left(\mathrm{W} \mathrm{kg}^{-1}\right)$ & 34.1 & 33.2 & 33.1 & 32.1 & 35.7 & 32.2 & 34.2 & 31.0 \\
\hline \hline
\end{tabular}

\subsection{Chapter Summaries}

In this study, realistic body and wing kinematics are reconstructed from high-speed video recordings of a dragonfly during turning maneuver. Numerical simulations are then used to investigate the aerodynamic functions and associated vortex formation of its two-pair wings. The wing kinematics analysis indicate that during the turn there are large asymmetries between the wing pitch angle and wing stroke angles especially for forewings, while asymmetries in wing deviation angle are generally small.

The asymmetrical wing kinematics generate unbalanced forces in both vertical and horizontal directions. During the downstroke, the force generated by the outer wings are higher than inner wings. By contrast, the inner wings generate higher forces during the upstroke. The surface pressure distribution shows that the majority of the force is generated around the leading- 
edge. The unbalanced forces lead to the torques along its body axis in three directions. Specifically, both forewings and hindwing contribute to the roll torque generation, whereas forewings are dominant in generating pitch and yaw torques. To examine the effect of forewinghindwing interaction on the aerodynamic performance, two additional cases, one with forewings only and the other with hindwings only, are carried out. Our results indicate that the interaction can lead to a slight forces reduction, but can save the aerodynamic power up to $11 \%$. 


\section{Concluding Remarks}

The works presented in the current dissertation have investigated vortex structures and aerodynamic performance of both simplified canonical models and realistic insect models by using computational fluid dynamics simulations. Results from the current studies aim to provide new physical insights into 3-D vortex development and wake topologies of low-aspect-ratio flapping wings with application to next generation MAVs.

\subsection{Summary of Contributions}

In Chapter 3, the effects of deformable flapping plates on unsteady aerodynamics were numerically studied at low Reynolds numbers. The chord-wise camber was modeled by a hinge connecting two rigid components. The leading portion was driven by a biological hovering motion along a horizontal stroke plane. The hinged trailing-edge flap (TEF) was controlled by a prescribed harmonic deflection motion. The effects of TEF deflection amplitude, deflection phase difference, hinge location, and Reynolds number on the aerodynamic performance and flow structures were investigated. The results demonstrated that the unsteady aerodynamic performance of deformable flapping plates was dominated by the TEF deflection phase difference, which directly affected the strength of the leading-edge vortex (LEV) and thus influenced the entire vortex shedding process. The overall lift enhancement reached $26 \%$ by tailoring the deflection amplitude and deflection phase difference. It was also found that the role of the dynamic TEF played in the flapping flight was consistent over a range of hinge locations and Reynolds numbers. Results from a low aspect-ratio $(A R=2)$ deformable plate showed the same trend as those of 2-D cases despite the effect of the three-dimensionality. The findings of 
this work have indicated an alternative solution to design the future dynamic deformed wings applied by MAVs.

In Chapter 4, the wake topology and propulsive performance of low-aspect-ratio plates undergoing a pitching-rolling motion in a uniform stream were numerically investigated. A detailed analysis of the vortical structures indicated that the pitching-rolling plate produced double-loop vortices with alternating signs from its trailing edge every half period. These vortices then shed and further evolved into interconnected "double-C"-shaped vortex rings, which eventually formed a bifurcating wake pattern in the downstream. As the wake convected downstream, there was a slight deflection in the spanwise direction to the plate tip, and the contained vortex ring size gradually increased. In addition, the analysis of the propulsive performance indicated that the shedding process of the double-loop vortices led to two peaks in the lift and thrust force production per half cycle. The observation of the double peaks in force production was in line with previous flapping wing studies. Simulations were also used to examine the variation of wake structures and propulsive performance of the plates over a range of major parameters. The aforementioned vortex structures were found to be quite robust over a range of Strouhal numbers, Reynolds numbers, and plate aspect ratios. This is the first report of unique vortical structures (“double-C" shaped vortex rings) generated by low-aspect-ratio pitching-rolling plates.

Chapter 6 used an integrated study combining high-speed photogrammetry with 3-D subdivision surface reconstruction and direct numerical simulation to study a dragonfly in forward flight. The flapping-wing kinematics and surface deformation, including both chordwise camber and spanwise twist, were obtained through the subdivision surface reconstruction method. We then explored the effects of deformable wings used the reconstructed wing model, first by 
removing camber while keeping the same time-varying twist distribution, and second by removing both the camber and the spanwise twist. The aerodynamic performance and vortex formation of these different wing models were explored via 3-D computational fluid dynamics simulations. To better understand the aerodynamic roles of deformable wings, the leading-edge vortex strength, the wing surface pressure distribution, and wake structures were analyzed and compared in detail. Our simulation results revealed that the spanwise twist can improve the aerodynamic functions in two ways: 1) improved the power economy by preventing the tip vortex bursting; and 2) improved the leading-edge vortex attachment by suppressing the generation of the secondary vortex. As a result, the spanwise twist could boost the aerodynamic efficiency by $20 \%$, especially during the wing translational phase. This is the first investigation of morphing wing effects on aerodynamic functions for a free flying dragonfly.

Chapter 7 studied dragonfly turning maneuver by combining high-speed photogrammetry, 3-D surface reconstruction, and direct numerical simulation. Quantitative measurements of wing kinematics demonstrated that during the turn, large asymmetries of wing stroke angle and wing pitch angle occurred between the left and right wings. During the downstroke flapping motion, the inner wings flap slower than the outer wings with a large angle of attack, and during the upstroke, they flap faster with a smaller angle of attack. The asymmetrical wing kinematics produce torques along the body axis in three directions. Unsteady aerodynamic calculations illustrated that both forewings and hindwing contribute to the roll torque generation, whereas forewings play a dominant role in generating pitch and yaw torques. 3-D vortex structures at selected instants were presented at the early stage of turning motion. Distinct vortex loops consist of the leading-edge vortex, tip vortex, and trailing-edge vortex were formed for each wing. The forewing-hindwing interaction effect was also studied. By comparing the aerodynamic 
performance of two-pair wings and isolated single-pair wings simulations, the wing-wing interaction effect could save up to $11 \%$ aerodynamic power along with slight force reduction. Results of the present work aim to provide insights into future agile quad-winged MAVs designs and applications.

\subsection{Future Work}

One possible extension of the current research includes exploring the effect of morphing wings using a flow-structure-interaction-based approach. This method could improve our understating of the passive mechanisms applied by natural creatures. To achieve this, a robust algorithm must be addressed for handling the solid and fluid continua coupling, since the solution should be convergent at every iterative step in both the solid and fluid domains. In addition, an accurate structure model for mimicking the material properties of real insect wings would be required to achieve a realistic surface morphing.

Another possible extensive study is to develop a robust reduced order modeling (ROM) based on the current proper orthogonal decomposition (POD) and force survey method (FSM). For a flapping wing, there are infinite degrees of freedom in high-fidelity numerical simulations. Evaluating the optimal kinematics and surface morphing using the direct numerical simulations is time consuming. Reduced-order models, on the other hand, abandon some detail flow information and add another level of approximation to the problem. This approach has the potential to achieve a quick evaluation of the complex unsteady flow. 


\section{Appendix A: CFD Solver Validations}

In order to demonstrate the validity of the numerical code, simulations of both 2-D and 3-D flapping wings were conducted compared with previous published results and experimental measurements. Overall, our simulation results showed a pretty well agreement with all published computational/experimental works.

\section{Two-dimensional flapping wing:}

The simulation results obtained for unsteady flow around a thin and rigid wing are compared with previously reported results $[80,81]$. In this test, the wing rotates around its center, whose orientation is measured counterclockwise relative to the positive $\mathrm{x}$-axis and the amplitude is denoted by $\beta$. To match the simulation setup, $A_{0} / c=2.8$ and $\beta=\pi / 4$ are chosen for the wing kinematics, and the Reynolds number, based on $U_{\max }$ and $c$, is 75 . For the simulation setup, the computational domain size is $30 c \times 40 c$ with a refined zone of $8 c \times 11 c$. The special grid $(321 \times 465)$ is chosen with the smallest resolution of $\Delta x=0.025 c$ in the dense uniform zone. The

flow is initially quiescent. The lift and drag coefficients for the first four cycles are shown in Figure A-1. It can be observed that the present simulation shows good agreement with both experimental measurements [80] and numerical results [81] obtained using a viscous vortex particle method (VVPM). 

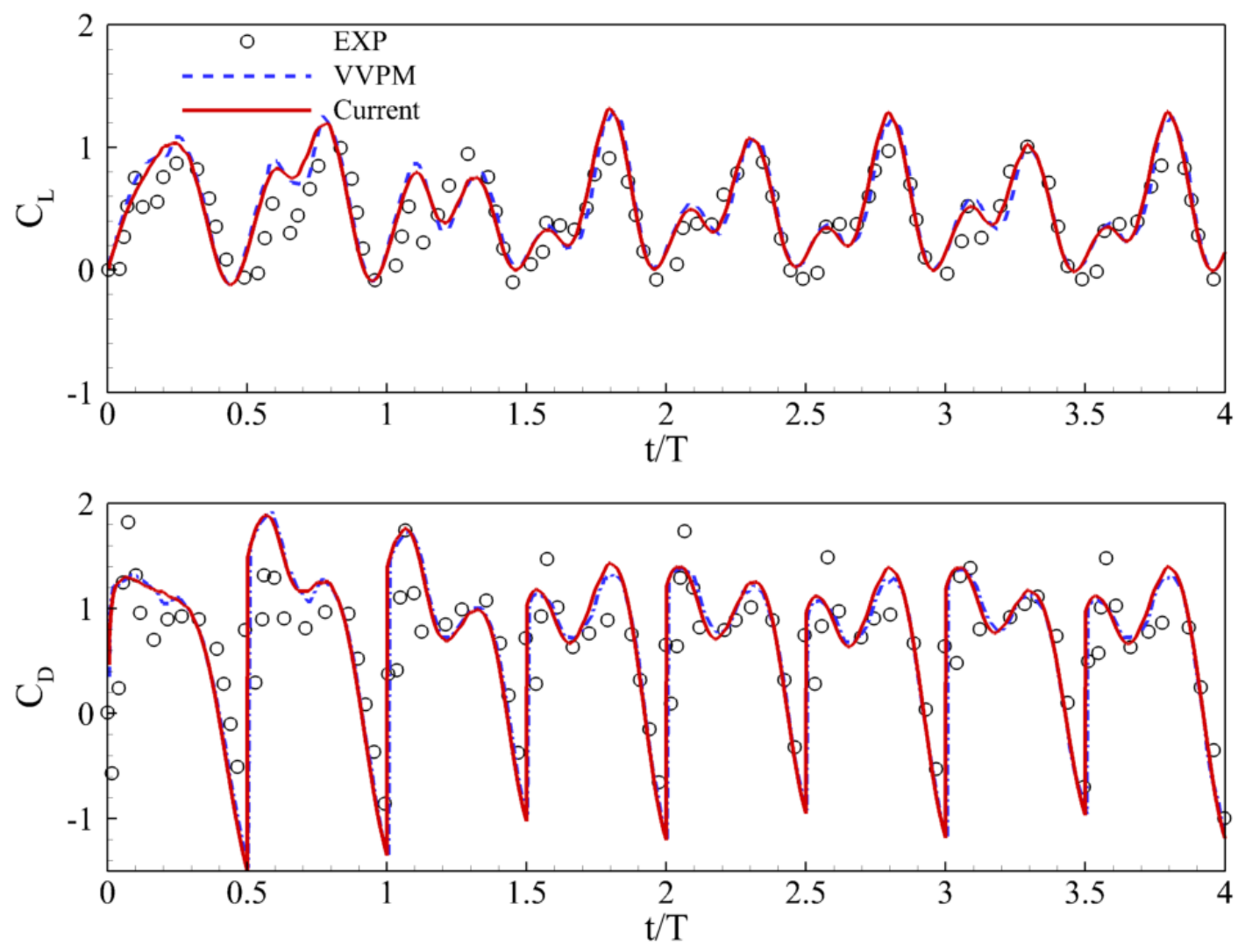

Figure A-1. Lift and drag coefficients from the current simulation, from experimental (EXP) measurement [80] and from a viscous vortex particle method (VVPM) simulation [81].

In Figure A-2, the wake development by showing the vorticity field at different instants are presented. The corresponding flow field from Ref. [81] is shown for comparison. It can be observed that the instantaneous vortex structures obtained from the two simulations agree with each other very well. 

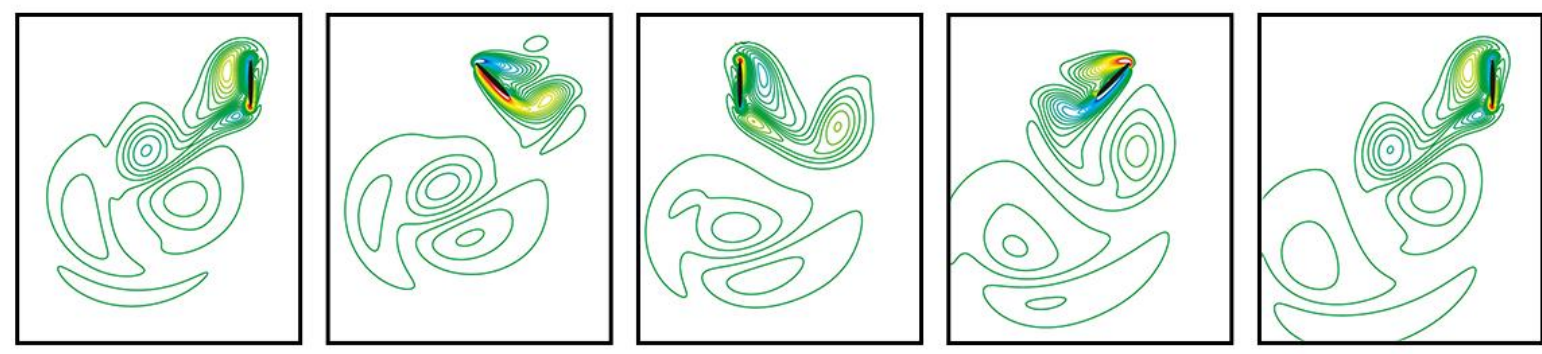

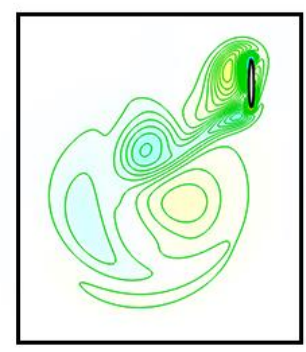

$\mathrm{t} / \mathrm{T}=3$

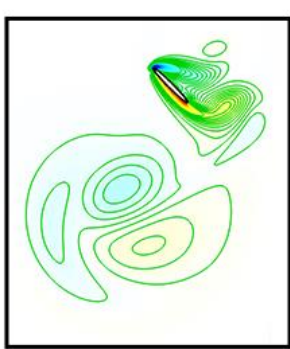

$\mathrm{t} / \mathrm{T}=3.25$

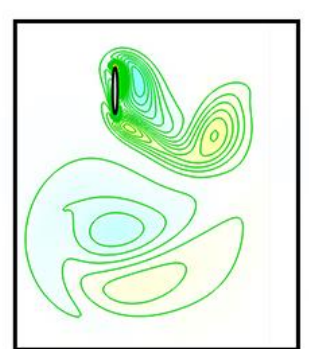

$\mathrm{t} / \mathrm{T}=3.5$

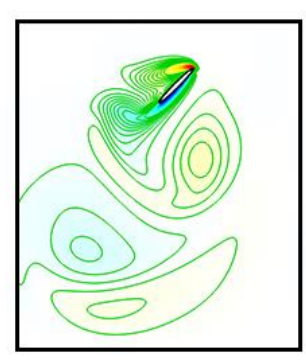

$\mathrm{t} / \mathrm{T}=3.75$

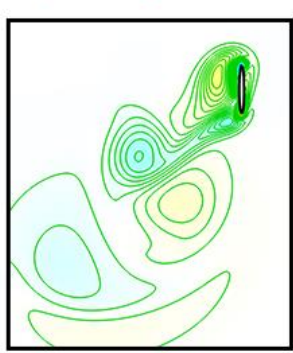

$\mathrm{t} / \mathrm{T}=4.0$

Figure A-2. Vorticity field at the labeled instants with 40 contour levels distributed uniformly between -20 and 20. The top row is from Ref. [81], and the bottom row is from the current simulation.

\section{Three-dimensional flapping wing:}

To demonstrate the validity of the current numerical solver in 3-D simulations, a simulation of flow around a robotic fruit fly wing was conducted. The robotic wing replicates Drosophila melanogaster wing with wing area $0.0167 \mathrm{~m}^{2}$, span $0.25 \mathrm{~m}$ and averaged chord $(\bar{c}) 8.79 \mathrm{~cm}$. The wing in experiments [130] sweeps in the horizontal plane and rotates at the end of each stroke. The stroke amplitude is $180^{\circ}$, the angle of attack at mid-stroke is $50^{\circ}$, and flapping frequency is $0.168 \mathrm{~Hz}$. The Reynolds number is 136 , with the average translational velocity at the wing tip $0.53 \mathrm{~m} \mathrm{~s}^{-1}$. A non-uniform Cartesian grid of size of $256 \times 144 \times 192$ is used in a computational domain of $30 \bar{c} \times 30 \bar{c} \times 30 \bar{c}$ to get domain independence results. The simulation is conducted for 
five flapping cycles. The drag and lift forces during each stroke is virtually identical after the third cycle. Figure A-3 shows the lift and drag force from the fifth cycle together with the previous experimental measurements [130] and numerical results [58]. The magnitude and trends with variation over time of the computed lift and drag forces are in reasonable good agreement with the previous results.

(a)

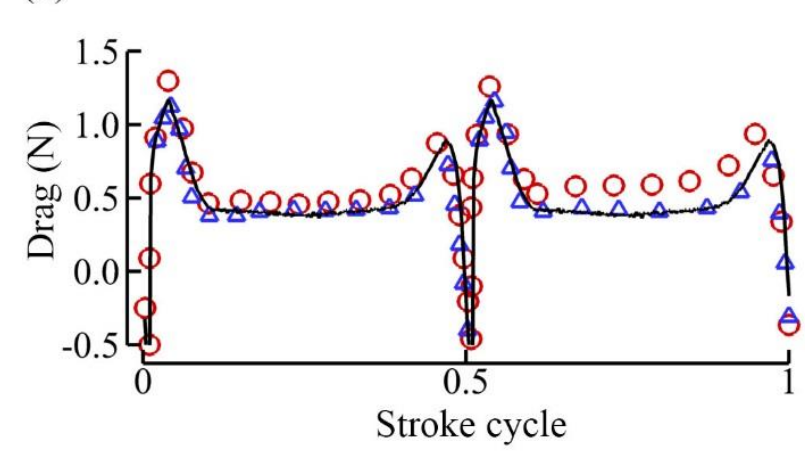

(b)

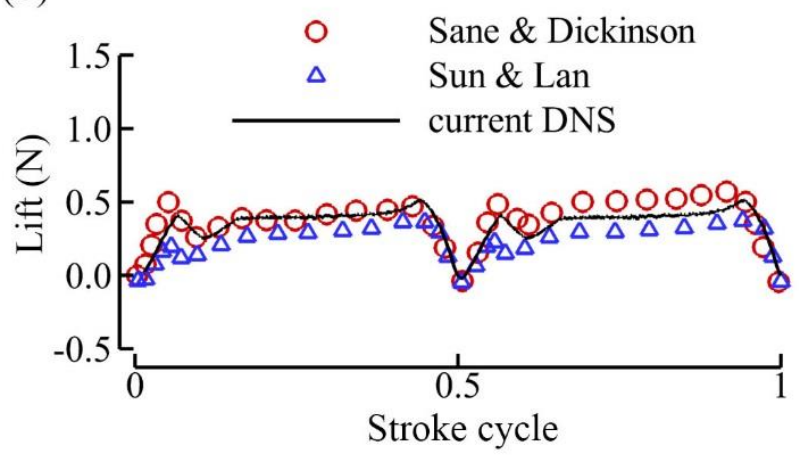

Figure A-3. Benchmarking of three-dimensional flapping simulation. The experimental and numerical data are reproduced from fig.3 C, D of Sane and Dickinson [130] and fig.4 C, D of Sun and Lan [58], respectively. 


\section{Bibliography}

[1] B.H. Carmichael, Low Reynolds Number Airfoil Survey, NASA Contractor Report (1981).

[2] A.a.M. Pelletier, T. J., Low Reynolds Number Aerodynamics of Low-Aspect-Ratio, Journal of Aircraft (2005) 825-832.

[3] A.R. Jones, H. Babinsky, Unsteady Lift Generation on Rotating Wings at Low Reynolds Numbers, Journal of Aircraft 47 (2010) 1013-1021.

[4] C.P. Ellington, C. vandenBerg, A.P. Willmott, A.L.R. Thomas, Leading-edge vortices in insect flight, Nature 384 (1996) 626-630.

[5] D. Lentink, M.H. Dickinson, Biofluiddynamic scaling of flapping, spinning and translating fins and wings, Journal of Experimental Biology 212 (2009) 2691-2704.

[6] G.V. Lauder, Aerodynamics: Flight of the robofly, Nature 412 (2001) 688-689.

[7] R. Dudley, The biomechanics of insect flight, Princeton University Press, 2000.

[8] M.M. Koochesfahani, Vortical Patterns in the Wake of an Oscillating Airfoil, AIAA Journal 27 (1989) 1200-1205.

[9] J.M. Anderson, K. Streitlien, D.S. Barrett, M.S. Triantafyllou, Oscillating foils of high propulsive efficiency, Journal of Fluid Mechanics 360 (1998) 41-72.

[10] R. Godoy-Diana, J.-L. Aider, J.E. Wesfreid, Transitions in the wake of a flapping foil, Physical Review E 77 (2008).

[11] I.H. Tuncer, M.F. Platzer, Computational study of flapping airfoil aerodynamics, Journal of Aircraft 37 (2000) 514-520.

[12] Z.J. Wang, Vortex shedding and frequency selection in flapping flight, Journal of Fluid Mechanics 410 (2000) 323-341.

[13] X. Zhu, G. He, X. Zhang, How flexibility affects the wake symmetry properties of a selfpropelled plunging foil, Journal of Fluid Mechanics 751 (2014) 164-183.

[14] M. Xu, M. Wei, C. Li, H. Dong, Adjoint-based optimization of flapping plates hinged with a trailing-edge flap, Theoretical and Applied Mechanics Letters 5 (2015) 1-4.

[15] C. Li, H. Dong, Y. Ren, A Numerical Study of Flapping Plates Hinged with a Trailing-Edge Flap, AIAA Paper 2014-2049 (2014). 
[16] R. Godoy-Diana, C. Marais, J.-L. Aider, J.E. Wesfreid, A model for the symmetry breaking of the reverse Benard-von Karman vortex street produced by a flapping foil, Journal of Fluid Mechanics 622 (2009) 23-32.

[17] D.J. Cleaver, Z. Wang, I. Gursul, Bifurcating flows of plunging aerofoils at high Strouhal numbers, Journal of Fluid Mechanics 708 (2012) 349-376.

[18] G.C. Lewin, H. Haj-Hariri, Modelling thrust generation of a two-dimensional heaving airfoil in a viscous flow, Journal of Fluid Mechanics 492 (2003) 339-362.

[19] X. Zhu, G. He, X. Zhang, Numerical study on hydrodynamic effect of flexibility in a selfpropelled plunging foil, Computers \& Fluids 97 (2014) 1-20.

[20] C. Li, H. Dong, G. Liu, Effects of a dynamic trailing-edge flap on the aerodynamic performance and flow structures in hovering flight, Journal of Fluids and Structures 58 (2015) 49-65.

[21] D. Calderon, D. Cleaver, I. Gursul, Z. Wang, On the absence of asymmetric wakes for periodically plunging finite wings, Physics of Fluids 26 (2014) 071907.

[22] K.D. Von Ellenrieder, K. Parker, J. Soria, Flow structures behind a heaving and pitching finite-span wing, Journal of Fluid Mechanics 490 (2003) 129-138.

[23] P. Blondeaux, F. Fornarelli, L. Guglielmini, M.S. Triantafyllou, R. Verzicco, Numerical experiments on flapping foils mimicking fish-like locomotion, Physics of Fluids 17 (2005).

[24] J.H.J. Buchholz, A.J. Smits, On the evolution of the wake structure produced by a lowaspect-ratio pitching panel, Journal of Fluid Mechanics 546 (2006) 433-443.

[25] J.H.J. Buchholz, A.J. Smits, The wake structure and thrust performance of a rigid lowaspect-ratio pitching panel, Journal of Fluid Mechanics 603 (2008) 331-365.

[26] H. Dong, R. Mittal, F.M. Najjar, Wake topology and hydrodynamic performance of lowaspect-ratio flapping foils, Journal of Fluid Mechanics 566 (2006) 309-343.

[27] Z. Liang, H. Dong, On the symmetry of proper orthogonal decomposition modes of a lowaspect-ratio plate, Physics of Fluids 27 (2015) 063601.

[28] M.S. Triantafyllou, A.H. Techet, F.S. Hover, Review of experimental work in biomimetic foils, IEEE Journal of Oceanic Engineering 29 (2004) 585-594.

[29] A.H. Techet, Propulsive performance of biologically inspired flapping foils at high Reynolds numbers, Journal of Experimental Biology 211 (2008) 274-279. 
[30] P.R. Bandyopadhyay, D.N. Beal, J.D. Hrubes, A. Mangalam, Relationship of roll and pitch oscillations in a fin flapping at transitional to high Reynolds number, Journal of Fluid Mechanics 702 (2012) 298-331.

[31] H. Dong, R. Mittal, M. Bozkurttas, G.V. Lauder, P. Madden, Computational Modeling and Analysis of the Hydrodynamics of a Highly Deformable Fish Pectoral Fin, Journal of Fluid Mechanics (2010).

[32] G. Liu, Y. Ren, J. Zhu, H. Bart-Smith, H. Dong, Thrust producing mechanisms in rayinspired underwater vehicle propulsion, Theoretical and Applied Mechanics Letters 5 (2015) 54-57.

[33] M. Bozkurttas, Hydrodynamic performance of fish pectoral fins with application to autonomous underwater vehicles, 2007.

[34] J. Valasek, Morphing aerospace vehicles and structures, Wiley, Chichester, 2012.

[35] H. Wang, L.J. Zeng, H. Liu, C.Y. Yin, Measuring wing kinematics, flight trajectory and body attitude during forward flight and turning maneuvers in dragonflies, Journal of Experimental Biology 206 (2003) 745-757.

[36] W. Shyy, Y. Liang, J. Tang, D. Viieru, H. Liu, Aerodynamics of Low Reynolds Number Flyers, Cambridge University Press, 2011.

[37] L. Zhao, X.Y. Deng, S.P. Sane, Modulation of leading edge vorticity and aerodynamic forces in flexible flapping wings, Bioinspiration \& Biomimetics 6 (2011).

[38] M.H. Dickinson, F.O. Lehmann, S.P. Sane, Wing rotation and the aerodynamic basis of insect flight, Science 284 (1999) 1954-1960.

[39] M. Sun, H. Tang, Unsteady aerodynamic force generation by a model fruit fly wing in flapping motion, Journal of Experimental Biology 205 (2002) 55-70.

[40] H. Dai, H. Luo, J.F. Doyle, Dynamic pitching of an elastic rectangular wing in hovering motion, Journal of Fluid Mechanics 693 (2012) 473-499.

[41] K. Shoele, Q. Zhu, Performance of a wing with nonuniform flexibility in hovering flight, Physics of Fluids 25 (2013).

[42] J. Toomey, J.D. Eldredge, Numerical and experimental study of the fluid dynamics of a flapping wing with low order flexibility, Physics of Fluids 20 (2008).

[43] J.D. Eldredge, J. Toomey, A. Medina, On the roles of chord-wise flexibility in a flapping wing with hovering kinematics, Journal of Fluid Mechanics 659 (2010) 94-115. 
[44] M. Vanella, T. Fitzgerald, S. Preidikman, E. Balaras, B. Balachandran, Influence of flexibility on the aerodynamic performance of a hovering wing, Journal of Experimental Biology 212 (2009) 95-105.

[45] H. Wan, H. Dong, G.P. Huang, Hovering Hinge-Connected Flapping Plate with Passive Deflection, AIAA Journal 50 (2012) 2020-2027.

[46] T. Lee, Y.Y. Su, Lift enhancement and flow structure of airfoil with joint trailing-edge flap and Gurney flap, Experiments in Fluids 50 (2011) 1671-1684.

[47] C.M. Ho, Y.C. Tai, Review: MEMS and its applications for flow control, Journal of Fluids Engineering-Transactions of the Asme 118 (1996) 437-447.

[48] T.A.W. Brian C. Prock, and William A. Crossley, Morphing Airfoil Shape Change Optimization with Minimum Actuator Energy as an Objective, 9th AIAA/ISSMO Symposium on Multidisciplinary Analysis and Optimization, Atlanta, Georgia, 2002.

[49] Y. Liu, B. Cheng, X. Deng, An experimental Study of Dynamic Trailing Edge Deflections on a Two Dimensional Translating Wing, 31st AIAA Applied Aerodynamics Conference, AIAA, 2013.

[50] J.M. Wakeling, Dragonfly aerodynamics and unsteady mechanisms: a review, Odonatologica 22 (1993) 319.

[51] J. Sun, C. Pan, J. Tong, J. Zhang, Coupled model analysis of the structure and nanomechanical properties of dragonfly wings, Nanobiotechnology, IET 4 (2010) 10-18.

[52] S. Lan, M. Sun, Aerodynamic interactions of two airfoils in unsteady motion, Acta mechanica 150 (2001) 39-51.

[53] L. Shilong, S. Mao, Aerodynamic force and flow structures of two airfoils in flapping motions, Acta Mechanica Sinica 17 (2001) 310-331.

[54] Wang, Effect of forewing and hindwing interactions on aerodynamic forces and power in hovering dragonfly flight, Physical Review Letters 99 (2007) 148101.

[55] A. Azuma, S. Azuma, I. Watanabe, T. Furuta, Flight Mechanics of a Dragonfly, Journal of Experimental Biology 116 (1985) 79-107.

[56] C.-T. Hsieh, C.-F. Kung, C.C. Chang, C.-C. Chu, Unsteady aerodynamics of dragonfly using a simple wing-wing model from the perspective of a force decomposition, Journal of Fluid Mechanics 663 (2010) 233-252. 
[57] T.M. Broering, Y. Lian, W. Henshaw, Numerical investigation of energy extraction in a tandem flapping wing configuration, AIAA journal 50 (2012) 2295-2307.

[58] M. Sun, S.L. Lan, A computational study of the aerodynamic forces and power requirements of dragonfly (Aeschna juncea) hovering, Journal of Experimental Biology 207 (2004) 1887-1901.

[59] R.A. Norberg, The Pterostigma of Insect Wings an Inertial Regulator of Wing Pitch, J. comp. Physiol. 81 (1972) 9-22.

[60] R.A. Norberg, Hovering flight of the dragonfly Aeschna juncea L., kinematics and aerodynamics, Swimming and flying in nature, Springer, 1975, pp. 763-781.

[61] J.K. Wang, M. Sun, A computational study of the aerodynamics and forewing-hindwing interaction of a model dragonfly in forward flight, Journal of Experimental Biology 208 (2005) 3785-3804.

[62] W.J. Maybury, F.-O. Lehmann, The fluid dynamics of flight control by kinematic phase lag variation between two robotic insect wings, Journal of Experimental Biology 207 (2004) 4707-4726.

[63] J.R. Usherwood, F.O. Lehmann, Phasing of dragonfly wings can improve aerodynamic efficiency by removing swirl, Journal of the Royal Society Interface 5 (2008) 1303-1307.

[64] Lehmann, Wing-Wake interaction reduces power consumption in insect tandem wings, Experiments in Fluids 46 (2009) 765.

[65] D. Rival, D. Schoenweitz, C. Tropea, Vortex interaction of tandem pitching and plunging plates: a two-dimensional model of hovering dragonfly-like flight, Bioinspiration \& biomimetics 6 (2011) 016008.

[66] Y. Zheng, Y. Wu, H. Tang, Force measurements of flexible tandem wings in hovering and forward flights, Bioinspiration \& biomimetics 10 (2015) 016021.

[67] Y. Zang, R.L. Street, J.R. Koseff, A non-staggered grid, fractional step method for timedependent incompressible Navier-Stokes equations in curvilinear coordinates, Journal of Computational Physics 114 (1994) 18-33.

[68] G.E. Schneider, M. Zedan, A modified strongly implicit procedure for the numerical solution of field problems, Numerical Heat Transfer 4 (1981) 1-19. 
[69] R. Mittal, H. Dong, M. Bozkurttas, F.M. Najjar, A. Vargas, A. von Loebbecke, A versatile sharp interface immersed boundary method for incompressible flows with complex boundaries, Journal of Computational Physics 227 (2008) 4825-4852.

[70] H. Wan, H. Dong, C. Li, Z. Liang, Vortex Formation and Aerodynamic Force of Low Aspect-Ratio Plate in Translation and Rotation, AIAA Paper 2012-3278 (2012).

[71] C. Li, H. Dong, Wake Structure and Aerodynamic Performance of Low Aspect-Ratio Revolving Plates at Low Reynolds number, AIAA Paper 2014-1453 (2014).

[72] C. Li, H. Dong, Z. Liang, Proper Orthogonal Decomposition Analysis of 3-D Wake Structures in a Pitching-Rolling Plate, AIAA Paper 2016-2071 (2016).

[73] G. Liu, H. Dong, C. Li, Vortex dynamics and new lift enhancement mechanism of wingbody interation in insect forward flight, Journal of Fluid Mechanics 795 (2016) 634-651.

[74] L. Sirovich, Turbulence and the dynamics of coherent structures. parts i-iii, Quarterly of Applied Mathematics 45 (1987) 561-571, 573-590.

[75] Z. Liang, H. Dong, Virtual Force Measurement of POD Modes for A Flat Plate in Low Reynolds Number Flows, AIAA Paper No.2014-0054 (2014).

[76] P. Gerontakos, T. Lee, PIV study of flow around unsteady airfoil with dynamic trailing-edge flap deflection, Experiments in fluids 45 (2008) 955-972.

[77] T. Lee, P. Gerontakos, Dynamic stall flow control via a trailing-edge flap, AIAA journal 44 (2006) 469-480.

[78] T. Lee, Y. Su, Unsteady airfoil with a harmonically deflected trailing-edge flap, Journal of Fluids and Structures 27 (2011) 1411-1424.

[79] S. Wang, X. Zhang, G. He, T. Liu, Lift enhancement by dynamically changing wingspan in forward flapping flight, Physics of Fluids 26 (2014) 061903.

[80] Z.J. Wang, J.M. Birch, M.H. Dickinson, Unsteady forces and flows in low Reynolds number hovering flight: two-dimensional computations vs robotic wing experiments, Journal of Experimental Biology 207 (2004) 449-460.

[81] J.D. Eldredge, Numerical simulation of the fluid dynamics of 2D rigid body motion with the vortex particle method, Journal of Computational Physics 221 (2007) 626-648.

[82] B. Yin, H.X. Luo, Effect of wing inertia on hovering performance of flexible flapping wings, Physics of Fluids 22 (2010). 
[83] P. Trizila, C.K. Kang, H. Aono, W. Shyy, M. Visbal, Low-Reynolds-Number Aerodynamics of a Flapping Rigid Flat Plate, AIAA Journal 49 (2011) 806-823.

[84] G. Du, M. Sun, Effects of unsteady deformation of flapping wing on its aerodynamic forces, Applied Mathematics and Mechanics-English Edition 29 (2008) 731-743.

[85] L. Zhao, Q.F. Huang, X.Y. Deng, S.P. Sane, Aerodynamic effects of flexibility in flapping wings, Journal of The Royal Society Interface 7 (2010) 485-497.

[86] A.J. Bergou, L. Ristroph, J. Guckenheimer, I. Cohen, Z.J. Wang, Fruit Flies Modulate Passive Wing Pitching to Generate In-Flight Turns, Physical Review Letters 104 (2010).

[87] C. Koehler, Z.X. Liang, Z. Gaston, H. Wan, H.B. Dong, 3D reconstruction and analysis of wing deformation in free-flying dragonflies, Journal of Experimental Biology 215 (2012) 3018-3027.

[88] S.M. Walker, A.L.R. Thomas, G.K. Taylor, Deformable wing kinematics in the desert locust: how and why do camber, twist and topography vary through the stroke?, Journal of the Royal Society Interface 6 (2009) 735-747.

[89] S.M. Walker, A.L.R. Thomas, G.K. Taylor, Deformable wing kinematics in free-flying hoverflies, Journal of the Royal Society Interface 7 (2010) 131-142.

[90] L. Zheng, T.L. Hedrick, R. Mittal, Time-varying wing-twist improves aerodynamic efficiency of forward flight in butterflies, PloS one 8 (2013) e53060.

[91] T.Q. Le, T. Van Truong, S.H. Park, T.Q. Truong, J.H. Ko, H. Cheol, Improvement of the aerodynamic performance by wing, (2013).

[92] G. Zhang, J. Sun, D. Chen, Y. Wang, Flapping motion measurement of honeybee bilateral wings using four virtual structured-light sensors, Sensors and Actuators A: Physical 148 (2008) 19-27.

[93] K. Taira, T.I.M. Colonius, Three-dimensional flows around low-aspect-ratio flat-plate wings at low Reynolds numbers, Journal of Fluid Mechanics 623 (2009) 187-207.

[94] J.C.R. Hunt, A.A. Wray, P. Moin, Eddies, streams, and convergence zones in turbulent flows, Center for Turbulence Research Report CTR-S88 (1988).

[95] W. Shyy, P. Trizila, C.K. Kang, H. Aono, Can Tip Vortices Enhance Lift of a Flapping Wing?, Aiaa Journal 47 (2009) 289-293.

[96] C. Wilga, G. Lauder, Locomotion in sturgeon: function of the pectoral fins, Journal of Experimental Biology 202 (1999) 2413-2432. 
[97] M. Bozkurttas, R. Mittal, H. Dong, G.V. Lauder, P. Madden, Low-dimensional models and performance scaling of a highly deformable fish pectoral fin, Journal of Fluid Mechanics 631 (2009) 311-342.

[98] K.W. Moored, P.A. Dewey, A.J. Smits, H. Haj-Hariri, Hydrodynamic wake resonance as an underlying principle of efficient unsteady propulsion, Journal of Fluid Mechanics 708 (2012) 329-348.

[99] H. Wan, H. Dong, K. Gai, Computational investigation of cicada aerodynamics in forward flight, Journal of The Royal Society Interface 12 (2015) 20141116.

[100] R.P. Clark, A.J. Smits, Thrust production and wake structure of a batoid-inspired oscillating fin, Journal of fluid mechanics 562 (2006) 415-429.

[101] A. Cain, R. Bush, Numerical wave propagation analysis for stretched grids, AIAA Paper (1994) 94-0172.

[102] M.A. Green, C.W. Rowley, A.J. Smits, The unsteady three-dimensional wake produced by a trapezoidal pitching panel, Journal of Fluid Mechanics 685 (2011) 117-145.

[103] D.B. Quinn, K.W. Moored, P.A. Dewey, A.J. Smits, Unsteady propulsion near a solid boundary, Journal of Fluid Mechanics 742 (2014) 152-170.

[104] M.H. Dickinson, K.G. Gotz, The wake dynamics and flight forces of the fruit fly Drosophila melanogaster, Journal of Experimental Biology 199 (1996) 2085-2104.

[105] S.P. Sane, M.H. Dickinson, The aerodynamic effects of wing rotation and a revised quasisteady model of flapping flight, Journal of Experimental Biology 205 (2002) 1087-1096.

[106] J.H. Wu, M. Sun, Unsteady aerodynamic forces of a flapping wing, Journal of Experimental Biology 207 (2004) 1137-1150.

[107] J.H. Wu, M. Sun, Unsteady aerodynamic forces and power requirements of a bumblebee in forward flight, Acta Mechanica Sinica 21 (2005) 207-217.

[108] R. Mittal, H. Dong, M. Bozkurttas, G. Lauder, P. Madden, Locomotion with flexible propulsors: II. Computational modeling of pectoral fin swimming in sunfish, Bioinspir Biomim 1 (2006) S35-41.

[109] H. Dong, M. Bozkurttas, R. Mittal, P. Madden, G.V. Lauder, Computational modelling and analysis of the hydrodynamics of a highly deformable fish pectoral fin, Journal of Fluid Mechanics 645 (2010) 345-373. 
[110] G.K. Taylor, R.L. Nudds, A.L.R. Thomas, Flying and swimming animals cruise at a Strouhal number tuned for high power efficiency, Nature 425 (2003) 707-711.

[111] J.J. Rohr, F.E. Fish, Strouhal numbers and optimization of swimming by odontocete cetaceans, Journal of Experimental Biology 207 (2004) 1633-1642.

[112] F.S. Hover, O. Haugsdal, M.S. Triantafyllou, Effect of angle of attack profiles in flapping foil propulsion, Journal of Fluids and Structures 19 (2004) 37-47.

[113] H. Soueid, L. Guglielmini, C. Airiau, A. Bottaro, Optimization of the motion of a flapping airfoil using sensitivity functions, Computers \& Fluids 38 (2009) 861-874.

[114] Z. Liang, H. Wan, H. Dong, P.S. Beran, Unsteady Flow and Its Reduced-Order Modeling of A Finite-Aspect-Ratio Flapping Foil, AIAA Paper, 2012.

[115] A.E. Deane, I.G. Kevrekidis, G.E. Karniadakis, O.S. A., Low-dimensional models for complex geometry flows: Application to grooved channels and circular cylinders, Physics of Fluids 3 (1991) 2337.

[116] I. Akhtar, A. Nayfeh, C. Ribbens, On the stability and extension of reduced-order Galerkin models in incompressible flows, Theoretical and Computational Fluid Dynamics 23 (2009) 213-237.

[117] T.Q. Le, T. Van Truong, S.H. Park, T.Q. Truong, J.H. Ko, H.C. Park, D. Byun, Improvement of the aerodynamic performance by wing flexibility and elytra-hind wing interaction of a beetle during forward flight, Journal of The Royal Society Interface 10 (2013) 20130312.

[118] J. Young, S.M. Walker, R.J. Bomphrey, G.K. Taylor, A.L.R. Thomas, Details of Insect Wing Design and Deformation Enhance Aerodynamic Function and Flight Efficiency, Science 325 (2009) 1549-1552.

[119] T.L. Hedrick, Software techniques for two- and three-dimensional kinematic measurements of biological and biomimetic systems, Bioinspiration \& Biomimetics 3 (2008).

[120] J. Wakeling, C. Ellington, Dragonfly flight. I. Gliding flight and steady-state aerodynamic forces, J Exp Biol 200 (1997) 543-556.

[121] G. Ruppell, Kinematic Analysis of Symmetrical Flight Maneuvers of Odonata, Journal of Experimental Biology 144 (1989) 13-42. 
[122] H. Dong, Z. Liang, H. Wan, C. Koehler, and Z. Gaston, An Integrated Analysis of a Dragonfly in Free Flight, 40th AIAA Fluid Dynamics Conference and Exhibit, 2010.

[123] A.E. Panah, J.M. Akkala, J.H. Buchholz, Vorticity transport and the leading-edge vortex of a plunging airfoil, Experiments in Fluids 56 (2015) 1-15.

[124] D.E. Alexander, Wind tunnel studies of turns by flying dragonflies, Journal of Experimental Biology 122 (1986) 81-98.

[125] H. Wang, L. Zeng, H. Liu, C. Yin, Measuring wing kinematics, flight trajectory and body attitude during forward flight and turning maneuvers in dragonflies, J Exp Biol 206 (2003) 745-757.

[126] B.a.R. Etkin, L. D., Dynamics of Flight: Stability and Control, New York: Wiley (1996).

[127] F.T. Muijres, M.J. Elzinga, J.M. Melis, M.H. Dickinson, Flies evade looming targets by executing rapid visually directed banked turns, Science 344 (2014) 172-177.

[128] L. Ristroph, G.J. Berman, A.J. Bergou, Z.J. Wang, I. Cohen, Automated hull reconstruction motion tracking (HRMT) applied to sideways maneuvers of free-flying insects, Journal of Experimental Biology 212 (2009) 1324-1335.

[129] F.O. Lehmann, When wings touch wakes: understanding locomotor force control by wakewing interference in insect wings, Journal of Experimental Biology 211 (2008) 224-233.

[130] S.P. Sane, M.H. Dickinson, The control of flight force by a flapping wing: lift and drag production, Journal of Experimental Biology 204 (2001) 3401-3401. 